\title{
Tackling Climate Change with Machine Learning
}

DAVID ROLNICK, McGill University and Mila - Quebec AI Institute

PRIYA L. DONTI, Carnegie Mellon University

LYNN H. KAACK, Hertie School and ETH Zürich

KELLY KOCHANSKI, University of Colorado Boulder

ALEXANDRE LACOSTE, Element AI/Service Now

KRIS SANKARAN, University of Wisconsin - Madison and Université de Montréal

ANDREW SLAVIN ROSS, New York University and Harvard University

NIKOLA MILOJEVIC-DUPONT, Mercator Research Institute on Global Commons

and Climate Change and Technische Universität Berlin

NATASHA JAQUES, Google Brain and UC Berkeley

ANNA WALDMAN-BROWN, Massachusetts Institute of Technology

ALEXANDRA SASHA LUCCIONI, Mila - Quebec AI Institute and Université de Montréal

TEGAN MAHARAJ, Mila - Quebec AI Institute and Polytechnique Montréal

EVAN D. SHERWIN, Stanford University

S. KARTHIK MUKKAVILLI, University of California and Lawrence Berkeley National Lab

KONRAD P. KORDING, University of Pennsylvania

CARLA P. GOMES, Cornell University

ANDREW Y. NG, Stanford University

DEMIS HASSABIS, DeepMind

JOHN C. PLATT, Google AI

FELIX CREUTZIG, Mercator Research Institute on Global Commons and Climate Change and Technische Universität Berlin

JENNIFER CHAYES, University of California, Berkeley

YOSHUA BENGIO, Mila - Quebec AI Institute and Université de Montréal

D.R. conceived and edited this work, with P.L.D., L.H.K., and K.K. Authors P.L.D., L.H.K., K.K., A.L., K.S., A.S.R., N.M-D., N.J., A.W-B., A.L., T.M., and E.D.S. researched and wrote individual sections. S.K.M., K.P.K., C.P.G., A.Y.N., D.H., J.C.P., F.C., J.C., and Y.B. contributed expert advice.

The authors gratefully acknowledge support from the National Science Foundation (grant no. 1803547), the Center for Climate and Energy Decision Making through a cooperative agreement between the National Science Foundation and Carnegie Mellon University (SES-00949710), US Department of Energy (contract DE-FG02-97ER25308), the Natural Sciences and Engineering Research Council of Canada, and the MIT Media Lab Consortium.

Authors' addresses: D. Rolnick; email: drolnick@cs.mcgill.ca; P. L. Donti; email: pdonti@andrew.cmu.edu; L. H. Kaack; email: kaack@hertie-school.org; K. Kochanski; email: kkochanski@alum.mit.edu; A. Lacoste; email: allac@elementai.com; K. Sankaran; email: ksankaran@wisc.edu; A. S. Ross; email: asross@nyu.edu; N. Milojevic-Dupont; email: milojevic@mccberlin.net; N. Jaques; email: natashajaques@google.com; A. Waldman-Brown; email: annawb@mit.edu; A. S. Luccioni and Y. Bengio; email: sashavluccioni@gmail.com; T. Maharaj; email: tegan.maharaj@mila.quebec; E. D. Sherwin and A. Y. Ng; emails: evands@stanford.edu, ang@cs.stanford.edu; S. K. Mukkavilli; email: karthik.mukkavilli@uci.edu; K. P. Kording; email: kording@seas.upenn.edu; C. P. Gomes; email: gomes@cs.cornell.edu; D. Hassabis; J. C. Platt; F. Creutzig; email: creutzig@mcc-berlin.net; J. Chayes.

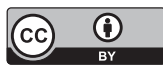

This work is licensed under a Creative Commons Attribution International 4.0 License.

(c) 2022 Copyright held by the owner/author(s).

0360-0300/2022/02-ART42

https://doi.org/10.1145/3485128

ACM Computing Surveys, Vol. 55, No. 2, Article 42. Publication date: February 2022. 
Climate change is one of the greatest challenges facing humanity, and we, as machine learning (ML) experts, may wonder how we can help. Here we describe how ML can be a powerful tool in reducing greenhouse gas emissions and helping society adapt to a changing climate. From smart grids to disaster management, we identify high impact problems where existing gaps can be filled by ML, in collaboration with other fields. Our recommendations encompass exciting research questions as well as promising business opportunities. We call on the ML community to join the global effort against climate change.

CCS Concepts: • General and reference $\rightarrow$ Surveys and overviews; $\bullet$ Computing methodologies $\rightarrow$ Machine learning; Artificial intelligence; • Applied computing $\rightarrow$ Operations research; Computers in other domains; Physical sciences and engineering;

Additional Key Words and Phrases: Climate change, mitigation, adaptation, machine learning, artificial intelligence

\section{ACM Reference format:}

David Rolnick, Priya L. Donti, Lynn H. Kaack, Kelly Kochanski, Alexandre Lacoste, Kris Sankaran, Andrew Slavin Ross, Nikola Milojevic-Dupont, Natasha Jaques, Anna Waldman-Brown, Alexandra Sasha Luccioni, Tegan Maharaj, Evan D. Sherwin, S. Karthik Mukkavilli, Konrad P. Kording, Carla P. Gomes, Andrew Y. Ng, Demis Hassabis, John C. Platt, Felix Creutzig, Jennifer Chayes, and Yoshua Bengio. 2022. Tackling Climate Change with Machine Learning. ACM Comput. Surv. 55, 2, Article 42 (February 2022), 96 pages. https://doi.org/10.1145/3485128

\section{INTRODUCTION}

The effects of climate change are increasingly visible. ${ }^{1}$ Storms, droughts, fires, and flooding have become stronger and more frequent [239]. Global ecosystems are changing, including the natural resources and agriculture on which humanity depends. The 2018 intergovernmental report on climate change estimated that the world will face catastrophic consequences unless global greenhouse gas (GHG) emissions are eliminated within 30 years [372]. Yet year after year, these emissions rise.

Addressing climate change involves mitigation (reducing emissions) and adaptation (preparing for unavoidable consequences). Both are multifaceted issues. Mitigation of GHG emissions requires changes to electricity systems, transportation, buildings, industry, and land use. Adaptation requires planning for resilience and disaster management, given an understanding of climate and extreme events. Such a diversity of problems can be seen as an opportunity: there are many ways to have an impact.

In recent years, machine learning (ML) has been recognized as a broadly powerful tool for technological progress. Despite the growth of movements applying ML and artificial intelligence (AI) to problems of societal and global good, ${ }^{2}$ there remains the need for a concerted effort to identify how these tools may best be applied to tackle climate change. Many ML practitioners wish to act, but are uncertain how. On the other side, many fields have begun actively seeking input from the ML community.

This article aims to provide an overview of where ML can be applied with high impact in the fight against climate change, through either effective engineering or innovative research. The strategies

\footnotetext{
${ }^{1}$ For a layman's introduction to the topic of climate change, see [32, 696].

${ }^{2}$ See the AI for social good movement (e.g., [71, 323]), ML for the developing world [163], the computational sustainability movement (e.g., [184, 296, 297, 401, 471], the American Meteorological Society's Committee on AI Applications to Environmental Science, and the field of Climate Informatics (www.climateinformatics.org.) [548], as well as the relevant survey papers [231, 251, 403].
} 
we highlight include climate mitigation and adaptation, as well as meta-level tools that enable other strategies. In order to maximize the relevance of our recommendations, we have consulted experts across many fields (see Acknowledgments) in the preparation of this article.

\subsection{Who is this Article Written For?}

We believe that our recommendations will prove valuable to several different audiences (detailed below). Given the wide diversity of technical areas involved, we do not assume any prior familiarity with application domains (such as agriculture or electric grids) and have tried to provide relevant keywords and background reading within each section of the article. While we use basic terminology from ML, knowledge of the specific ML techniques we reference is not necessary to understand any of our key points. For an overall introduction to ML, see e.g., [78].

Researchers and engineers: We identify many problems that require conceptual innovation and can advance the field of ML, as well as being highly impactful. For example, we highlight how climate models afford an exciting domain for interpretable ML (see Section 8.1). We encourage researchers and engineers across fields to use their expertise in solving urgent problems relevant to society.

Entrepreneurs and investors: We identify many problems where existing ML techniques could have a major impact without further research, and where the missing piece is deployment. We realize that some of the recommendations we offer here will make valuable startups and nonprofits. For example, we highlight techniques for providing fine-grained solar forecasts for power companies (see Section 2.1), tools for helping reduce personal energy consumption (see Section 11.2), and predictions for the financial impacts of climate change (see Section 14). We encourage entrepreneurs and investors to fill what is currently a wide-open space.

Corporate leaders: We identify problems where ML can lead to massive efficiency gains if adopted at scale by corporate players. For example, we highlight means of optimizing supply chains to reduce waste (see Section 5.1) and software/hardware tools for precision agriculture (see Section 6.2). We encourage corporate leaders to take advantage of opportunities offered by ML to benefit both the world and the bottom line.

Local and national governments: We identify problems where ML can improve public services, help gather data for decision-making, and guide plans for future development. For example, we highlight intelligent transportation systems (see Section 3.4), techniques for automatically assessing the energy consumption of buildings in cities (see Section 4.2), and tools for improving disaster management (see Section 9.4). We encourage policymakers to consider opportunities for working with ML experts and building ML capacity in relevant public sector bodies. We further encourage public entities to release data that may be relevant to climate change mitigation and adaptation goals. (For further policy-related recommendations on this topic, see e.g., [406].)

\subsection{How to Read this Article}

The article is broken into sections according to application domain (see Table 1). To help the reader, we have also included the following flags at the level of individual strategies.

- High Leverage denotes bottlenecks that domain experts have identified in climate change mitigation or adaptation and that we believe to be particularly well-suited to tools from ML. These areas may be especially fruitful for ML practitioners wishing to have an outsized impact, though applications not marked with this flag are also valuable and should be pursued. 
- Long-term denotes applications that will have their primary impact after 2040. While extremely important, these may in some cases be less pressing than those which can help act on climate change in the near term.

- Uncertain Impact denotes applications where the impact on GHG emissions is uncertain (for example, rebound effects may apply ${ }^{3}$ ) or where there is potential for undesirable side effects (negative externalities).

These flags should not be taken as definitive; they represent our understanding of more rigorous analyses within the domains we consider, combined with our subjective evaluation of the potential role of ML in these various applications.

Despite the length of the article, we cannot cover everything. There will certainly be many applications that we have not considered, or that we have erroneously dismissed. We look forward to seeing where future work leads.

\subsection{A Call for Collaboration}

All of the problems we highlight in this article require collaboration across fields. Collaboration reduces the chance of failure modes such as working on a problem that is not actually impactful, overly simplifying a complicated issue, or using advanced computational tools when simple tools will do the job.

Collaboration is also essential to ensure that innovations will be deployed with the intended impact. Relevant stakeholders should be involved in the full pipeline of problem scoping and development, so that the final solution is well-tailored to the setting in which it will be used. For example, code can be written using a language and a platform that are already popular with the intended users, or can be integrated into an existing, widely used tool.

We realize that finding partners, as well as relevant resources such as data, can often be difficult. We encourage readers to visit the website that accompanies this article, www.climatechange.ai, where we offer additional resources, as well as opportunities for knowledge-sharing and networking.

\subsection{The Broader Picture}

We emphasize that ML is not a silver bullet. The applications we highlight are impactful, but no one solution will "fix" climate change. There are also many areas of action where ML is inapplicable, and we omit these entirely. Moreover, while we focus here on ways in which ML can help address climate change, ML can also be applied in ways that make climate change worse. For instance, ML is used widely to accelerate activities such as fossil fuel exploration and extraction [303, 406, 814], while some ML models are themselves energy-intensive to train and run [69, 459, 720, 764]. ${ }^{4}$

Finally, technology is not in itself enough to solve climate change, nor is it a replacement for other aspects of climate action such as policy. Many technological tools useful in addressing climate change have been available for years but have yet to be adopted at scale by society. While we hope that ML will be useful in accelerating effective strategies for climate action, humanity also must decide to act.

\footnotetext{
${ }^{3}$ Rebound effects occur when increased efficiency results in higher demand, partially or completely negating the benefits of efficiency gains [45]. For example, lowering the energy required to produce a product can lead to lower costs, which in turn can increase the consumption of the product. In such cases, specific policies, such as pricing mechanisms or caps on GHG emissions, can help to limit rebound effects. See also the literature on induced demand and the Jevons paradox.

${ }^{4}$ It is worth noting that many ML methods cited in this article require only minimal energy to train and run (e.g., can be run on a laptop or phone).
} 
Table 1. Climate Change Solution Domains, Corresponding to Sections of this Article, Matched with Selected Areas of ML that are Relevant to Each

\begin{tabular}{|c|c|c|c|c|c|c|c|c|c|c|}
\hline & & 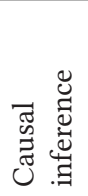 & 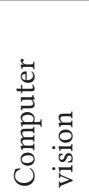 & 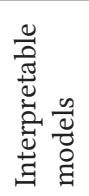 & 完 & $\begin{array}{l}\overrightarrow{0} \\
\text { : } \\
0 \\
\infty \\
\infty \\
\vec{\imath}\end{array}$ & 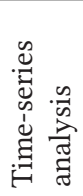 & 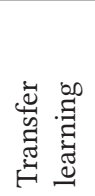 & 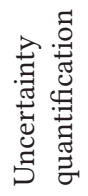 & 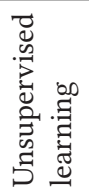 \\
\hline & Electricity systems & & & & & & & & & \\
\hline & Enabling low-carbon electricity & & - & - & & - & - & & - & - \\
\hline & Reducing current-system impacts & & - & & & & • & & - & • \\
\hline & Ensuring global impact & & - & & & & & - & & - \\
\hline & Transportation & & & & & & & & & \\
\hline & Reducing transport activity & & - & & & & - & & - & - \\
\hline & Improving vehicle efficiency & & $\bullet$ & & & $\bullet$ & & & & \\
\hline & Alternative fuels \& electrification & & & & & - & & & & - \\
\hline & Modal shift & - & - & & & & - & & - & \\
\hline & Buildings and cities & & & & & & & & & \\
\hline & Optimizing buildings & - & & & & - & - & - & & \\
\hline$\cong$ & Urban planning & & - & & & & - & - & & - \\
\hline .00 & The future of cities & & & & - & & & - & - & - \\
\hline & Industry & & & & & & & & & \\
\hline & Optimizing supply chains & & - & & & - & - & & & \\
\hline & Improving materials & & & & & & & & & - \\
\hline & Production \& energy & & - & - & & - & & & & \\
\hline & Farms \& forests & & & & & & & & & \\
\hline & Remote sensing of emissions & & - & & & & & & & \\
\hline & Precision agriculture & & - & & & - & - & & & \\
\hline & Monitoring peatlands & & - & & & & & & & \\
\hline & Managing forests & & - & & & - & - & & & \\
\hline & Carbon dioxide removal & & & & & & & & & \\
\hline & Direct air capture & & & & & & & & & - \\
\hline & Sequestering $\mathrm{CO}_{2}$ & & - & & & & & & - & - \\
\hline & Climate prediction & & & & & & & & & \\
\hline & Uniting data, ML \& climate science & & - & - & & & - & & - & \\
\hline & Forecasting extreme events & & - & - & & & - & & - & \\
\hline & Societal impacts & & & & & & & & & \\
\hline .0 & Ecology & & - & & & & & - & & \\
\hline है & Infrastructure & & & & & - & - & & - & \\
\hline$\overline{\text { สิ }}$ & Social systems & & - & & & & - & & & - \\
\hline 定 & Crisis & & - & & - & & & & & \\
\hline & Solar geoengineering & & & & & & & & & \\
\hline & Understanding \& improving aerosols & & & & & & - & & - & \\
\hline & Engineering a control system & & & & & - & & & - & \\
\hline & Modeling impacts & & & & & & - & & - & \\
\hline & Individual action & & & & & & & & & \\
\hline ๑ే & $\begin{array}{l}\text { Understanding personal footprint } \\
\text { Facilitating behavior change }\end{array}$ & $\bullet$ & & & $\bullet$ & $\bullet$ & $\bullet$ & & & $\bullet$ \\
\hline 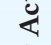 & Collective decisions & & & & & & & & & \\
\hline ö & Modeling social interactions & & & • & & - & & & & \\
\hline$\overline{0}$ & Informing policy & - & - & & - & & & & - & - \\
\hline$\because$ & Designing markets & & & & & - & - & & & - \\
\hline & Education & & & & - & - & & & & \\
\hline & Finance & & & & - & & - & & - & \\
\hline
\end{tabular}




\section{ELECTRICITY SYSTEMS}

BY PRIYA L. DONTI

AI has been called the new electricity, given its potential to transform entire industries [591]. Interestingly, electricity itself is one of the industries that AI is poised to transform. Many electricity systems are awash in data, and the industry has begun to envision next-generation systems (smart grids) driven by AI and ML [559, 623, 661, 814].

Electricity systems ${ }^{5}$ are responsible for about a quarter of human-caused GHG emissions each year [370]. Moreover, as buildings, transportation, and other sectors seek to replace GHG-emitting fuels (Section 3-4), demand for low-carbon electricity will grow. To reduce emissions from electricity systems, society must

- Rapidly transition to low-carbon ${ }^{6}$ electricity sources (such as solar, wind, hydro, and nuclear) and phase out carbon-emitting sources (such as coal, natural gas, and other fossil fuels).

- Reduce GHG emissions associated with existing fossil fuel and electricity infrastructure, since the transition to low-carbon power will not happen overnight.

- Implement these changes across all countries and contexts, as electricity systems are everywhere.

ML can contribute on all fronts by informing the research, deployment, and operation of electricity system technologies (Figure 1). Such contributions include accelerating the development of clean energy technologies, improving forecasts of demand and clean energy, improving electricity system optimization, and enhancing system monitoring. These contributions require a variety of ML paradigms and techniques, as well as close collaborations with the electricity industry and other experts to integrate insights from operations research, electrical engineering, physics, chemistry, the social sciences, and other fields.

\subsection{Enabling Low-Carbon Electricity}

Low-carbon electricity sources are essential to tackling climate change. These sources come in two forms: variable and controllable. Variable sources fluctuate based on external factors; for instance, solar panels produce power only when the sun is shining, and wind turbines only when the wind is blowing. On the other hand, controllable sources such as nuclear or geothermal plants can be turned on and off (though not instantaneously ${ }^{7}$ ). These two types of sources affect electricity systems differently, presenting distinct opportunities for ML techniques.

2.1.1 Variable Sources. Most electricity is delivered to consumers using a physical network called the electric grid, where the power generated must equal the power consumed at every moment. This means that solar panels, wind turbines, and other variable electricity generators are supported by some mix of natural gas plants, storage, or other controllable sources ready to buffer changes in their output (e.g., when unexpected clouds block the sun or the wind blows less strongly than predicted). Today, this buffer is often provided by coal and natural gas plants run in a $\mathrm{CO}_{2}$ emitting standby mode called spinning reserve. In the future, this role is expected to be played by

\footnotetext{
${ }^{5}$ Throughout this section, we use the term "electricity systems" to refer to the procurement of fuels and raw materials for electric grid components; the generation and storage of electricity; and the delivery of electricity to end-use consumers. For primers on these topics, see [96, 141, 437, 817, 848].

${ }^{6}$ We use the term "low-carbon" here instead of "renewable" because of this article's explicit focus on climate change goals Renewable energy is produced from inexhaustible or easily replenished energy sources such as the sun, wind, or water, but need not necessarily be carbon-free (as in the case of some biomass [149]). Similarly, not all low-carbon energy is renewable (as in the case of nuclear energy).

${ }^{7}$ Nuclear power plants are often viewed as inflexible since they can take hours or days to turn on or off, and are often left on (at full capacity) to operate as baseload. That said, nuclear power plants may have some flexibility to change their power generation for load-following and other electric grid services, as in the case of France [491].
} 


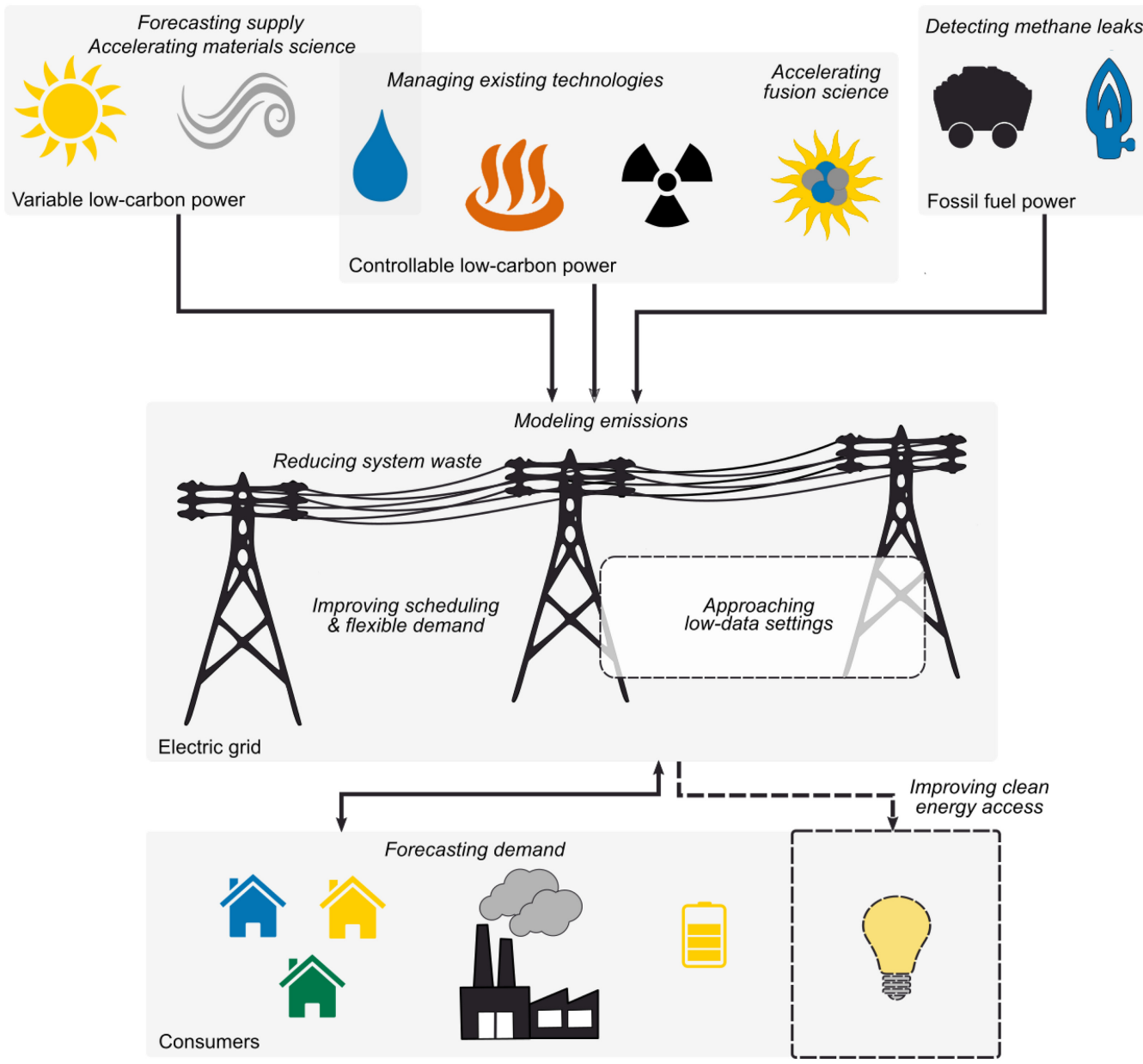

Fig. 1. Selected opportunities to reduce GHG emissions from electricity systems using ML, as described in Section 2.

energy storage technologies such as batteries (Section 3.3), pumped hydro, or power-to-gas [226]; see [27] for an overview. ${ }^{8}$ ML can both reduce emissions from today's standby generators and enable the transition to carbon-free systems by helping improve necessary technologies (namely forecasting, scheduling, and control) and by helping create advanced electricity markets that accommodate both variable electricity and flexible demand.

\section{Forecasting supply and demand.}

High Leverage

Since variable generation and electricity demand both fluctuate, they must be forecast ahead of time to inform real-time electricity scheduling and longer-term system planning. Better shortterm forecasts can allow system operators to reduce their reliance on polluting standby plants and to proactively manage increasing amounts of variable sources. Better long-term forecasts can help system operators (and investors) determine where and when variable plants should be built.

\footnotetext{
${ }^{8}$ It is worth noting that in systems with many fossil fuel plants, storage may increase emissions depending on how it is operated [47, 347].
} 
While many system operators today use basic forecasting techniques, forecasts will need to become increasingly accurate, span multiple horizons in time and space, and better quantify uncertainty to support these use cases. ML can help on all these fronts.

To date, many ML methods have been used to forecast electricity supply and demand. These methods have employed historical data, physical model outputs, images, and even video data to create short- to medium-term forecasts of solar power [9, 19,161, 478, 524, 771, 818], wind power [216, 285, 450, 512], "run-of-the-river" hydro power [623], demand [351, 429, 458, 673], or more than one of these $[402,857]$ at aggregate spatial scales. These methods span various types of supervised ML, fuzzy logic, and hybrid physical models, and take different approaches to quantifying (or not quantifying) uncertainty. At a more spatially granular level, some work has attempted to understand specific categories of demand, for instance by clustering households [63, 421] or by disaggregating electricity signals using game theory, optimization, regression, and/or online learning $[24,409,473]$.

While much of this previous work has used domain-agnostic techniques, ML algorithms of the future will need to incorporate domain-specific insights. For instance, since weather fundamentally drives both variable generation and electricity demand, ML algorithms forecasting these quantities should draw from innovations in climate modeling and weather forecasting (Section 8) and in hybrid physics-plus-ML modeling techniques [161, 818, 822]. Such techniques can help improve short- to medium-term forecasts, and are also necessary for ML to contribute to longer-term (e.g., year-scale) forecasts since weather distributions shift over time [404]. In addition to incorporating system physics, ML models should also directly optimize for system goals [198, 220, 842]. For instance, the authors of [198] use a deep neural network to produce demand forecasts that optimize for electricity scheduling costs rather than forecast accuracy; this notion could be extended to produce forecasts that minimize GHG emissions. In non-automated settings where power system control engineers (partially) determine how much power each generator should produce, interpretable ML and automated visualization techniques could help engineers better understand forecasts and thus improve how they schedule low-carbon generators. More broadly, understanding the domain value of improved forecasts is an interesting challenge. For example, previous work has characterized the benefits of specific solar forecast improvements in a region of the United States [523]; further study in different contexts and for different types of improvements could help better direct ML work in the forecasting space.

\section{Improving scheduling and flexible demand.}

When balancing electricity systems, system operators use a process called scheduling and dispatch to determine how much power every controllable generator should produce. This process is slow and complex, as it is governed by NP-hard optimization problems such as unit commitment and optimal power flow that must be coordinated across multiple time scales (from sub-second to days ahead). Further, scheduling will become even more complex as electricity systems include more storage, variable generators, and flexible demand, since operators will need to manage even more system components while simultaneously solving scheduling problems more quickly to account for real-time variations in electricity production. Scheduling processes must therefore improve significantly for operators to manage systems with a high reliance on variable sources.

ML can help improve the existing (centralized) process of scheduling and dispatch by speeding up power system optimization problems and improving the quality of optimization solutions [333, 608]. For instance, ML can be used to approximate or simplify existing optimization problems [75, 242, 311, 871], find good starting points for optimization [52, 196, 382], identify redundant constraints [541], learn from the actions of power system control engineers [197], or do some combination of these [858]. Dynamic scheduling [225, 546] and (safe) reinforcement learning 
(RL) could also be used to balance the electric grid in real time; in fact, some electricity system operators have started to pilot similar methods at small, test case-based scales [520].

While many modern electricity systems are centrally coordinated, recent work has examined how to (at least partially) decentralize scheduling and dispatch using energy storage, flexible demand, low-carbon generators, and other resources connected to the electric grid. One strategy is to explicitly design local control algorithms; for instance, recent work has controlled energy storage and solar inverters using supervised learning techniques trained on historical optimization data [191, 192, 410, 411]. Another strategy is to let storage, demand, and generation respond to real-time prices ${ }^{9}$ that reflect (for example) how emissions-intensive electricity currently is. In this case, ML can help both to design real-time prices and to respond to these prices. Previous work has used RL and dynamic programming to set real-time electricity prices [30, 90] and more broadly for power market design [30, 881]. Techniques such as (deep) RL [291, 292, 810, 881], agent-based models [173, 662, 663, 863], online optimization [99], and dynamic programming [704] can then help maximize profits for decentralized storage, demand, and generation, given real-time prices. See also [30] for an overview of deep learning techniques for demand response. In general, much more work is needed to test and scale existing decentralized solutions; barring deployment on real systems, platforms such as PowerTAC [638] and Grid2Op [519] can provide large-scale simulated environments on which to perform these tests.

\section{Accelerating materials science.}

\begin{tabular}{|l|l|}
\hline High Leverage & Long-term \\
\hline
\end{tabular}

Scientists are working to develop new materials that can better store or otherwise harness energy from variable natural resources. For instance, creating solar fuels (synthetic fuels produced from sunlight or solar heat) could allow us to capture solar energy when the sun is shining and then store this energy for later use. However, the process of discovering new materials can be slow and imprecise; the physics behind materials are not completely understood, so human experts often manually apply heuristics to understand a proposed material's physical properties $[49,105]$. ML can automate this process by combining existing heuristics with experimental data, physics, and reasoning to apply and even extend existing physical knowledge. For instance, recent work has used tools from ML, AI, optimization, and physics to figure out a proposed material's crystal structure, with the goal of accelerating materials discovery for solar fuels [49, 298, 773]. Other work seeking to improve battery storage technologies has combined first-principles physics calculations with support-vector regression to design conducting solids for lithium-ion batteries [257]. (Additional applications of ML to batteries are discussed in Section 3.3.) Recent work has also proposed the use of ML for scalable simulation of electrocatalysts for power-to-gas applications [894].

More generally in materials science, ML techniques including supervised learning, active learning, and generative models have been used to help synthesize, characterize, model, and design materials, as described in reviews [105, 490] and more recent work [299]. As discussed in [105], novel challenges for ML in materials science include coping with moderately sized datasets and inferring physical principles from trained models [800]. In addition to advancing technology, ML can inform policy for accelerated materials science; for instance, previous work has applied natural language processing to patent data to understand the solar panel innovation process [813]. We note that while our focus here has been on electricity system applications, ML for accelerated science may also have significant impacts outside electricity systems, e.g., by helping design alternatives to cement (Section 5.2) or create better $\mathrm{CO}_{2}$ sorbents (Section 7.1).

\footnotetext{
${ }^{9}$ For discussions and examples of different types of advanced electricity markets, see [109, 483, 484, 877].
} 


\section{Additional applications.}

There are many additional opportunities for ML to advance variable power generation. For instance, it is important to ensure that low-carbon variable generators produce energy as efficiently and profitably as possible. Prior work has attempted to maximize electricity production by controlling solar panels $[1,3,678]$ or wind turbine blades [2, 833] using RL or Bayesian optimization. Other work has used graphical models to detect faults in rooftop solar panels [377] and genetic algorithms to optimally place wind turbines within a wind farm [188]. ML can also help control batteries located at solar and wind farms to increase these farms' profits, for instance by storing their electricity when prices are low and then selling it when prices are high; prior work has used ML to forecast electricity prices $[460,838]$ or RL to control batteries based on current and historical prices [825].

ML can also help integrate rooftop solar panels into the electric grid, particularly in the United States and Europe. Rooftop solar panels are connected to a part of the electric grid called the distribution grid, which traditionally did not have many sensors because it was only used to deliver electricity "one-way" from centralized power plants to consumers. However, rooftop solar and other distributed energy resources have created a "two-way" flow of electricity on distribution grids. Since the locations and sizes of rooftop solar panels are often unknown to electricity system operators, previous work has used computer vision techniques on satellite imagery to generate size and location data for rooftop solar panels [165, 514, 868]. Further, to ensure that the distribution system runs smoothly, recent work has employed techniques such as matrix completion and deep neural networks to estimate the state of the system when there are few sensors [199, 388, 626].

2.1.2 Controllable Sources. Controllable low-carbon electricity sources can help achieve climate change goals while requiring very few changes to how the electric grid is run (since today's fossil fuel power plants are also controllable). ML can support existing controllable technologies while accelerating the development of new technologies such as nuclear fusion power plants.

Managing existing technologies.

Many controllable low-carbon technologies are already commercially available; these technologies include geothermal, nuclear fission, and (in some cases ${ }^{10}$ ) dam-based hydropower. ML can provide valuable input in planning where these technologies should be deployed and can also help maintain already-operating power plants. For instance, recent work has proposed to use ML to identify and manage sites for geothermal energy, using satellite imagery and seismic data [593]. Previous work has also used multi-objective optimization to place hydropower dams in a way that satisfies both energy and ecological objectives [856]. Finally, ML can help maintain nuclear fission reactors (i.e., nuclear power plants) by detecting cracks and anomalies from image and video data [129] or by preemptively detecting faults from high-dimensional sensor and simulation data [107]. (The authors of [794] speculate that ML and high performance computing could also be used to help simulate nuclear waste disposal options or even design next-generation nuclear reactors.)

Accelerating fusion science.

High Leverage Long-term

Nuclear fusion reactors [577] have the potential to produce safe and carbon-free electricity using a virtually limitless hydrogen fuel supply, but currently consume more energy than they produce [146]. While considerable scientific and engineering research is still needed, ML can help accelerate this work by, e.g., guiding experimental design and monitoring physical processes; see also [360]. Fusion reactors require intelligent experimental design because they have a large number

\footnotetext{
${ }^{10}$ Dam-based hydropower may produce methane, primarily due to biomass that decomposes when a hydro reservoir floods, but the amount produced varies between power plants [753]
} 
of tunable parameters; ML can help prioritize which parameter configurations should be explored during physical experiments. For instance, Google and TAE Technologies have developed a humanin-the-loop experimental design algorithm enabling rapid parameter exploration for TAE's reactor [53].

Physically monitoring fusion reactors is also an important application for ML. Modern reactors attempt to super-heat hydrogen into a plasma state and then stabilize it, but during this process, the plasma may experience rapid instabilities that damage the reactor. Prior work has tried to preemptively detect disruptions for tokamak reactors, using supervised learning methods such as supportvector machines, adaptive fuzzy logic, decision trees, and deep learning [110, 413, 567, 811, 846, 850] on previous disruption data. While many of these methods are tuned to work on individual reactors, recent work has shown that deep learning may enable insights that generalize to multiple reactors [413]. More generally, rather than simply detecting disruptions, scientists need to understand how plasma's state evolves over time, e.g., by finding the solutions of time-dependent magnetohydrodynamic equations [57]; speculatively, ML could help characterize this evolution and even help steer plasma into safe states through reactor control. ML models for such fusion applications would likely employ a combination of simulated ${ }^{11}$ and experimental data, and would need to account for the different physical characteristics, data volumes, and simulator speeds or accuracies associated with different reactor types.

\subsection{Reducing Current-System Impacts}

While switching to low-carbon electricity sources will be essential, in the meantime, it will also be important to mitigate emissions from the electricity system as it currently stands. Some methods for mitigating current-system impacts include cutting emissions from fossil fuels, reducing waste from electricity delivery, and flexibly managing demand to minimize its emissions impacts.

Reducing life-cycle fossil fuel emissions.

High Leverage Uncertain Impact Reducing emissions from fossil fuels is a necessary stopgap while society transitions towards low-carbon electricity. In particular, ML can help prevent the leakage of methane (an extremely potent GHG) from natural gas pipelines and compressor stations. Previous and ongoing work has used sensor and/or satellite data to proactively suggest pipeline maintenance $[213,898]$ or detect existing leaks [81, 749, 823, 826], and there is a great deal of opportunity in this space to improve and scale existing strategies. In addition to leak detection, ML can help reduce emissions from freight transportation of solid fuels (Section 3), identify and manage storage sites for $\mathrm{CO}_{2}$ sequestered from power plant flue gas (Section 7.2), and optimize power plant parameters to reduce $\mathrm{CO}_{2}$ emissions. In all these cases, projects should be pursued with great care so as not to impede or prolong the transition to a low-carbon electricity system; ideally, projects should be preceded by system impact analyses to ensure that they will indeed decrease GHG emissions.

Reducing system waste.

As electricity gets transported from generators to consumers, some of it gets lost as resistive heat on electricity lines. While some of these losses are unavoidable, others can be significantly mitigated to reduce waste and emissions. ML can help prevent avoidable losses through predictive maintenance, e.g., by suggesting proactive electricity grid upgrades (see also Sections 5.3 and 9.2). Prior work has performed predictive maintenance using long short-term memory (LSTM) [76], bipartite ranking [702], and neural network-plus-clustering techniques [581] on electric grid data, and future work will need to improve and/or localize these approaches to different contexts.

${ }^{11}$ Plasma simulation frameworks for tokamak reactors include RAPTOR [236, 237], ASTRA [624], CRONOS [35], PTRANSP [100], and IPS [250]. 


\section{Modeling emissions.}

Flexibly managing household, commercial, industrial, and electric vehicle (EV) demand (as well as energy storage) can help minimize electricity-based emissions (Sections 3, 4, 5, and 11), but doing so involves understanding what the emissions on the electric grid actually are at any moment. Specifically, marginal emissions factors capture the emissions effects of small changes in demand at any given time. To inform consumers about marginal emissions factors, initiatives such as WattTime [832] and the electricityMap project [789] have used ML and regression-based techniques to forecast marginal emissions based on electricity and weather data. Recent work has also used ML for trend extraction and feature selection within marginal emissions forecasting models [476] Great Britain's National Grid ESO uses ensemble models to forecast average emissions factors, which measure the aggregate emissions intensity of all power plants [576]. There is still much room to improve the performance of these methods, as well as to forecast related quantities such as electricity curtailments (i.e., the wasting of usually low-carbon electricity for grid balancing purposes). As most existing methods produce point estimates, it would also be important to quantify the uncertainty of these estimates to ensure that load-shifting techniques indeed decrease (rather than increase) emissions.

\subsection{Ensuring Global Impact}

Much of the discussion around electricity systems often focuses on settings such as the United States with near universal electricity access and relatively abundant data. However, many places that do not share these attributes are still integral to tackling climate change [370] and warrant serious consideration. To ensure global impact, ML can help improve electricity access and translate electricity system insights from high-data to low-data contexts.

Improving clean energy access.

Improving access to clean electricity can address climate change while simultaneously improving social and economic development [430,431]. Specifically, clean electricity provided via electric grids, microgrids, or off-grid methods can displace diesel generators, wood-burning stoves, and other carbon-emitting energy sources. Figuring out what clean electrification methods are best for different areas can require intensive, on-the-ground surveying work, but ML can help provide input to this process in a scalable manner. For instance, previous work has used image processing, clustering, and optimization techniques on satellite imagery to inform electrification initiatives [219]. ML can also help operate rural microgrids through accurate forecasts of demand and power production [120,601] as well as tailored optimization and control schemes [493], since small microgrids are even harder to balance than country-scale electric grids. Generating data to aid energy access policy and better managing energy access strategies are therefore two areas in which ML may have promising applications.

\section{Approaching low-data settings.}

High Leverage

While ML methods have often been applied to grids with widespread sensors, system operators in many countries do not collect or share system data. Although these data availability practices may evolve, it may meanwhile be beneficial to use ML techniques such as transfer learning to translate insights from high-data to low-data settings (especially since all electric grids share the same underlying system physics). Developing data-efficient ML techniques will likely also be useful in low-data settings; for instance, in [680], the authors enforce physical or other domain-specific constraints on weakly supervised ML models, allowing these models to learn from very little labeled data.

ML can also help generate information within low-data settings. For instance, recent work has estimated the layout of electricity grids in regions where they are not explicitly mapped, using 
computer vision and graph search techniques on satellite imagery [278, 359]. Companies have also used satellite imagery to measure power plant $\mathrm{CO}_{2}$ emissions [114, 301] (also see Section 6.1). Other recent work has modeled electricity consumption using regression-based techniques on cellular network data [85], which may prove useful in settings with many cellular towers but few electric grid sensors. Although low-data settings are generally underexplored by the ML community, electricity systems research in these settings presents opportunities for both innovative ML and climate change mitigation.

\subsection{Discussion}

Data-driven and critical to climate change, electricity systems hold many opportunities for ML research and practice. At the same time, applications in this space hold many potential pitfalls; for instance, innovations that seek to reduce GHG emissions in the oil and gas industries could actually increase emissions by making them cheaper to emit [814]. Given these domain-specific nuances, working in this area requires close collaborations with electricity system decision-makers and with practitioners in fields including electrical engineering, the natural sciences, and the social sciences. Interpretable ML may also enable practitioners to better understand, apply, and audit models in real-world settings. Similarly, it will be important to develop hybrid ML models that explicitly account for system physics (see e.g., [132, 164, 304, 680, 843]), directly optimize for domain-specific goals [198, 220, 842], or otherwise incorporate or scale existing domain knowledge. Finally, since many modern electric grids are not data-abundant (although they may be data-driven), understanding how to apply data-driven insights to these grids may be the next grand challenge for ML in electricity systems.

\section{TRANSPORTATION}

BY LYNN H. KAACK

Transportation systems form a complex web that is fundamental to an active and prosperous society. Globally, the transportation sector accounts for about a quarter of energy-related $\mathrm{CO}_{2}$ emissions [372]. In contrast to the electricity sector, however, transportation has not made significant progress to lower its $\mathrm{CO}_{2}$ emissions [154] and much of the sector is regarded as hard to decarbonize [162]. This is because of the high energy density of fuels required for many types of vehicles, which constrains low-carbon alternatives, and because transport policies directly impact end-users and are thus more likely to be controversial.

Passenger and freight transportation are each responsible for about half of transport GHG emissions [712]. Both freight and passengers can travel by road, by rail, by water, or by air (referred to as transport modes). Different modes carry vastly different carbon emission intensities. ${ }^{12}$ At present, more than two-thirds of transportation emissions are from road travel [712], but air travel has the highest emission intensity and is responsible for an increasingly large share. Strategies to reduce GHG emissions ${ }^{13}$ from transportation consist of [712]:

- reducing transport activity;

- improving vehicle efficiency;

- alternative fuels and electrification; and

- modal shift (shifting to lower-carbon options, like rail).

Each of these mitigation strategies offers opportunities for ML (Figure 2). While many of us probably think of autonomous vehicles (AVs) and ride-sharing when we think of transport and

\footnotetext{
${ }^{12}$ Carbon intensity is measured in grams of $\mathrm{CO}_{2}$-equivalent per person-km or per ton-km, respectively.

${ }^{13}$ For general resources on how to decarbonize the transportation sector, see the AR5 chapter on transportation [712], and $[240,407,784]$.
} 

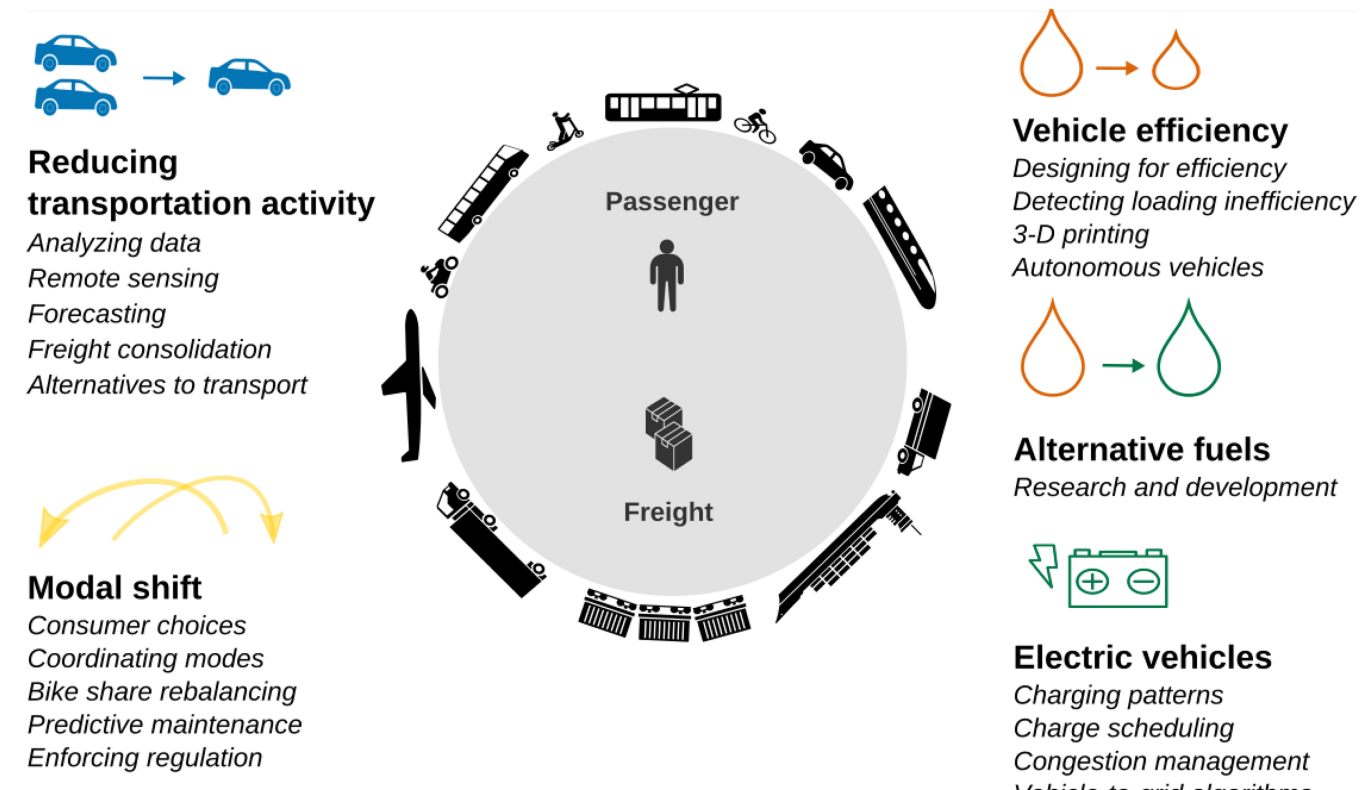

Alternative fuels

Research and development
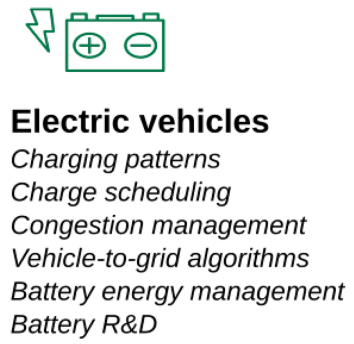

Fig. 2. Selected opportunities to reduce GHG emissions from transportation using ML, as described in Section 3.

ML, these technologies have uncertain impacts on GHG emissions [820], potentially even increasing them. We discuss these disruptive technologies in Section 3.1 but show that ML can play a role for decarbonizing transportation that goes much further. ML can improve vehicle engineering, enable intelligent infrastructure, and provide policy-relevant information. Many interventions that reduce GHG emissions in the transportation sector require changes in planning, maintenance, and operations of transportation systems, even though the GHG reduction potential of those measures might not be immediately apparent. ML can help in implementing such interventions, for example, by providing better demand forecasts. Typically, ML strategies are most effective in tandem with strong public policies. While we do not cover all ML applications in the transportation sector, we aim to include those areas that can conceivably reduce GHG emissions.

\subsection{Reducing Transport Activity}

A colossal amount of transport occurs each day across the world, but much of this mileage occurs inefficiently, resulting in needless GHG emissions. With the help of ML, the number of vehiclemiles traveled can be reduced by making long trips less necessary, increasing loading, and optimizing vehicle routing. Here, we discuss the first two in depth-for a discussion of ML and routing, see for example [873].

Understanding transportation data.

Many areas of transportation lack data, and decision-makers often design infrastructure and policy with uncertain information. In recent years, new types of sensors have become available, and ML can turn this raw data into useful information. Traditionally, traffic is monitored with groundbased counters that are installed on selected roads. A variety of technologies are used, such as 
inductive loop detectors or pneumatic tubes. Traffic is sometimes monitored with video systems, in particular when counting pedestrians and cyclists, which can be automated with computer vision [870]. Since counts on most roads are often available only over short time frames, these roads are modeled by looking at known traffic patterns for similar roads. ML methods, such as SVMs and neural networks, have made it easier to classify roads with similar traffic patterns $[270,454,796]$. As ground-based counters require costly installation and maintenance, many countries do not have such systems. Vehicles can also be detected in high-resolution satellite images with high accuracy [177, 390, 566, 747], and image counts can serve to estimate average vehicle traffic [405] Similarly, ML methods can help with imputing missing data for precise bottom-up estimation of GHG emissions [583] and they are also applied in simulation models of vehicle emissions [46]

\section{Modeling demand.}

High Leverage

Modeling demand and planning new infrastructure can significantly shape how long trips are and which transport modes are chosen by passengers and shippers-for example, discouraging sprawl and creating new transportation links can both reduce GHG emissions. ML can provide information about mobility patterns, which is directly necessary for agent-based travel demand models, one of the main transport planning tools [864]. For example, ML makes it possible to estimate origin-destination demand from traffic counts [504], and it offers new methods for spatio-temporal road traffic forecasting-which do not always outperform other statistical methods [223] but may transfer well between areas [776]. Also, short-term forecasting of public transit ridership can improve with ML; see for example $[158,586]$. ML is particularly relevant for deducing information from novel data-for example, learning about the behavior of public transit users from smart card data [280, 515]. Also, mobile phone sensors provide new means to understand personal travel demand and the urban topology, such as walking route choices [795]. Similarly, ML-based modeling of demand can help mitigate climate change by improving operational efficiency of modes that emit significant $\mathrm{CO}_{2}$, such as aviation. $\mathrm{ML}$ can help predict runway demand and aircraft taxi time in order to reduce the excess fuel burned in the air and on the ground due to congestion in airports $[379,474]$.

\section{Shared mobility.}

Uncertain Impact

In the passenger sector, shared mobility (such as on-demand ride services or vehicle-sharing ${ }^{14}$ ), is undoubtedly disrupting the way people travel and think about vehicle ownership, and ML plays an integral part in running and optimizing these services (e.g., [768, 837]). However, it is largely unclear what the impact of this development will be on GHG emissions. For example, shared cars can actually cause more people to travel by car, as opposed to using public transportation. Similarly, on-demand taxi services add mileage when traveling without a customer, possibly negating any GHG emission savings [765]. On the other hand, shared mobility can lead to higher utilization of each vehicle, which means a more efficient use of materials [341]. The use of newer and more efficient vehicles, ideally electric ones, could increase with vehicle-sharing concepts, reducing GHG emissions. Some of the issues raised above could also perhaps be overcome by making taxis autonomous. Such vehicles also might integrate better with public transportation, and offer new concepts for pooled rides, which substantially reduce the emissions per person-mile.

ML methods can help to understand the energy impact of shared mobility concepts. For example, they can be used to predict if a customer decides to share a ride with other passengers from an ondemand ride service [133]. For decision-makers it is important to have access to timely locationspecific empirical analysis to understand if a ride share service is taking away customers from

\footnotetext{
${ }^{14}$ In this section, we discuss shared cars; see Section 3.4 for bike shares and electric scooters.
} 
low-carbon transit modes and increasing the use of cars. Some local governments are beginning to require data-sharing from these providers (see Section 4.3).

Car-sharing services using AVs could yield GHG emission savings when they encourage people to use public transit for part of the journey [552] or with autonomous EVs [408]. However, using autonomous shared vehicles alone could increase the total vehicle-miles traveled and therefore do not necessarily lead to lower emissions as long as the vehicles have internal combustion engines (or electrical engines on a "dirty" electrical grid) [131, 495]. We see the intersection of shared mobility, autonomous and EVs, and smart public transit-prioritizing low-carbon vehicle technologies and shared transportation-as a path where ML can make a contribution to shaping future mobility See also Section 3.2 for more on AVs.

When designing and promoting new mobility services, it is important that industry and public policy prioritize lowering GHG emissions. Misaligned incentives in the early stages of technological development could result in the lock-in to a service with high GHG emissions [36, 44].

\section{Freight routing and consolidation.}

High Leverage

Bundling shipments together, which is referred to as freight consolidation, dramatically reduces the number of trips (and therefore the GHG emissions). The same is true for changing routing so that trucks do not have to return empty. As rail and water modes require much larger loads than trucks, consolidation also enables shipments to use these modes for part of the journey [407]. Freight consolidation and routing decisions are often taken by third-party logistics service providers and other freight forwarders, such as in the less-than-truckload market, which deals with shipments of smaller sizes. ML offers opportunities to optimize this complex interaction of shipment sizes, modes, origin-destination pairs, and service requirements. Many problem settings are addressed with methods from the field of operations research. There is evidence that ML can improve upon these methods, in particular mixed-integer linear programming [70]. Other proposed and deployed applications of ML include predicting arrival times or demand, identifying and planning around transportation disruptions [279], and clustering suppliers by their geographical location and common shipping destinations. Proposed planning approaches include designing allocation algorithms and freight auctions, and ML has for example been shown to help pick good algorithms and parameters to solve auction markets [708].

Alternatives to transport.

Uncertain Impact Disruptive technologies that are based on ML could replace or reduce transportation demand. For example, additive manufacturing (AM) or 3-D printing has (limited) potential to reduce freight transport by producing lighter goods and enabling production closer to the consumer [407]. ML can be a valuable tool for improving AM processes [859]. ML can also help to improve virtual communication [733]. If passenger trips are replaced by telepresence, travel demand can be reduced, as has been shown for example in public agencies [34] and for scientific teams [518]. However, it is uncertain to what extent virtual meetings replace physical travel, or if they may actually give rise to more face-to-face meetings [762].

\subsection{Improving Vehicle Efficiency}

Most vehicles are not very efficient compared to what is technically possible: for example, aircraft carbon intensity is expected to decline by more than a third with respect to 2012 , simply by virtue of newer models replacing aging jets [713]. Both the design of the vehicle and the way it is operated can increase the fuel economy. Here, we discuss how ML can help design more efficient vehicles and the impacts that autonomous driving may have on GHG emissions. Encouraging drivers to 
adopt more efficient vehicles is also a priority; while we do not focus on this here, ML plays a role in studying consumer preferences in vehicle markets [103].

\section{Designing for efficiency.}

There are many ways to reduce the energy a vehicle uses-such as more efficient engines, improved aerodynamics, hybrid electric engines, and reducing the vehicle's weight or tire resistance. These different strategies require a broad range of engineering techniques, many of which can benefit from ML. For example, ML is applied in advanced combustion engine design [384]. Hybrid EVs, which are more efficient than combustion engines alone, rely on power management methods that can be improved with ML [15]. Aerodynamic efficiency improvements need turbulence modeling that is often computationally intensive and relies heavily on ML-based surrogate models [865]. Aerodynamic improvements can not only be made by vehicle design but also by rearranging load. Lai et al. [461] use computer vision to detect aerodynamically inefficient loading on freight trains. AM (3-D printing) can produce lighter parts in vehicles, such as road vehicles and aircraft, that reduce energy consumption [341, 407]. ML is applied to improve those processes, for example through failure detection [722, 730] or material design [309].

Autonomous vehicles.

Uncertain Impact ML is essential in the development of AVs, including in such basic tasks as following the road and detecting obstacles [87]. ${ }^{15}$ While AVs could reduce energy consumption-for example, by reducing traffic congestion and inducing efficiency through eco-driving-it is also possible that AVs will lead to an increase in overall road traffic that nullifies efficiency gains. (For an overview of possible energy impacts of AVs, see [95, 820], and for broader impacts on mobility, see [327].) Two advantages of AVs in the freight sector promise to cut GHG emissions: First, small AVs, such as delivery robots and drones, could reduce the energy consumption of last-mile delivery [758], though they come with regulatory challenges [517]. Second, trucks can reduce energy consumption by platooning (driving very close together to reduce air resistance), thereby alleviating some of the challenges that come with electrifying long-distance road freight [318]. Platooning relies on autonomous driving and communication technologies that allow vehicles to brake and accelerate simultaneously.

ML can help to develop AV technologies specifically aimed at reducing energy consumption. For example, $\mathrm{Wu}$ et al. [851, 852] develop AV controllers based on RL to smooth out traffic involving non-AVs. Their studies of emergent behaviors in mixed-autonomy environments aim to understand the impact that varying shares of AVs can have on potentially reducing congestion-related energy consumption ML methods can also help to understand driving practices that are more energy efficient. For example, Jiménez et al. [392] use data from smart phone sensors to identify driving behavior that leads to higher energy consumption in EVs.

\subsection{Alternative Fuels and Electrification}

Electric vehicles.

High Leverage EV technologies-using batteries, hydrogen fuel cells, or electrified roads and railways-are regarded as a primary means to decarbonize transport. EVs can have very low GHG emissionsdepending, of course, on the carbon intensity of the electricity. ML is vital for a range of different problems related to EVs. Rigas et al. [684] detail methods by which ML can improve charge scheduling, congestion management, and vehicle-to-grid algorithms. ML methods have also been applied

${ }^{15}$ Providing details on the general role of ML for AVs is beyond the scope of this article. 
to battery energy management (for example charge estimation [330] or optimization in hybrid EVs [15]), and to detect faults and lateral misalignment in wireless charging of EVs [780].

As more people drive EVs, understanding their use patterns will become more important. Modeling charging behavior will be useful for grid operators looking to predict electric load. For this application, it is possible to analyze residential EV charging behavior from aggregate electricity load (energy disaggregation, see also Section 4.1) [827]. Also, in-vehicle sensors and communication data are increasingly becoming available and offer an opportunity to understand travel and charging behavior of EV owners, which can for example inform the placement of charging stations [779] or alternatives such as battery swapping stations.

Battery EVs are typically not used for more than a fraction of the day, allowing them to act as energy storage for the grid at other times, where charging and discharging is controlled for example by price signals [265] (see Sections 2.1.1 and 2.2). There is much potential for ML (e.g., RL [810]) to improve such vehicle-to-grid technology, which, like other mechanisms for grid energy storage (see Section 2.1.1), can help to reduce GHG emissions from electricity generation. Vehicle-to-grid technology comes with private and social financial benefits. However, consumers are expected to be reluctant to agree to such services, as they might not want to compromise their driving range [343].

Finally, ML can also play a role in the research and development of batteries, a decisive technology for EV costs and usability. Work in this area has focused on predicting battery state, degradation, and remaining lifetime using supervised learning techniques, fuzzy logic, and clustering $[42,104,218,358,414,725,735,819,854]$. However, many models developed in academia are based on laboratory data that do not account for real-world factors such as environmental conditions $[735,819,854]$. By contrast, industry lags behind in ML modeling, but real-world operational data are readily available. Merging these two perspectives could yield significant benefits for the field.

Alternative fuels.

Long-term

Much of the transportation sector is highly dependent on liquid fossil fuels. Aviation, long-distance road transportation, and ocean shipping require fuels with high energy density and thus are not conducive to electrification [162]. Electrofuels [98], solar fuels (Section 2.1.1), biofuels [6], hydrogen [111, 803], and perhaps natural gas [792] offer alternatives, but the use of these fuels is constrained by factors such as cost, land-use, and (for hydrogen and natural gas) incompatibility with current infrastructure [162]. Electrofuels and biofuels have the potential to serve as low-carbon drop-in fuels that retain the properties of fossil fuels, such as high energy density, while retaining compatibility with the existing fleet of vehicles and the current fuel infrastructure [407]. Fuels such as electrofuels and hydrogen can be produced using electricity-intensive processes and can be stored at lower cost than electricity. Thus, as a form of energy storage, these fuels could provide services to the electricity grid by enabling flexible power use and balancing variable electricity generators (Section 2.1.1). Given their relative long-term importance and early stage of development, they present a critical opportunity to mitigate climate change. ML techniques may present opportunities for improvement at various stages of research and development of alternative fuels (similar to applications in Section 2.1.1).

\subsection{Modal Shift}

Shifting passengers and freight to low carbon-intensity modes is one of the most important means to decarbonize transport. This modal shift in passenger transportation can for example involve providing people with public transit, which requires analyzing mode choice and travel demand data. ML can also make low-carbon freight modes more competitive by helping to coordinate intermodal transport. 


\section{Passenger preferences.}

ML can improve our understanding about passengers' travel mode choices, which in turn informs transportation planning, such as where public transit should be built. Some recent studies have shown that supervised ML based on survey data can improve passenger mode choice models $[322,571,597]$. Seo et al. propose to conduct long-term travel surveys with online learning, which reduces the demand on respondents, while obtaining high data quality [724]. Sun et al. [770] use SVMs and neural networks for analyzing preferences of customers traveling by high speed rail in China. There is also work on inferring people's travel modes and destinations from social media or various mobile phone sensors such as GPS (transportation mode detection), e.g., [156, 798]. Also in the freight sector, ML has been applied to analyze modal trade-offs, for example, by imputing data on counterfactual mode choices [706].

\section{Enabling low-carbon options.}

High Leverage

In order to incentivize more users to choose low-carbon transport modes, their costs and service quality can be improved. Many low-carbon modes must be integrated with other modes of transportation to deliver the same level of service. For example, when traveling by train, the trip to and from the station will often be by car, taxi, bus, or bike. There are many opportunities for ML to facilitate a better integration of modes, both in the passenger and freight sectors. ML can also help to improve the operation of low-carbon modes, for example, by reducing the operations and maintenance costs of rail [383] and predicting track degradation [746].

Bike sharing and electric scooter services can offer low-carbon alternatives for urban mobility that do not require ownership and integrate well with public transportation. ML studies help to understand how usage patterns for bike stations depend on their immediate urban surroundings [364]. ML can also help solve the bike sharing rebalancing problem, where shared bikes accumulate in one location and are lacking in other locations, by improving forecasts of bike demand and inventory [675]. Singla et al. [739] propose a pricing mechanism based on online learning to provide monetary incentives for bike users to help rebalancing. By producing accurate travel time estimates, ML can provide tools that help to integrate bike shares with other modes of transportation [281]. Many emerging bike and scooter sharing services are dockless, which means that they are parked anywhere in public space and can block sidewalks [25]. ML has been applied to monitor public sentiment about such bike shares via tweets [775]. ML could also provide tools and information for regulators to ensure that public space can be used by everyone [741].

Coordination between modes resulting in faster and more reliable transit times could increase the amount of people or goods traveling on low-carbon modes such as rail. ML algorithms could be applied to make public transportation faster and easier to use. For example, there is a rich literature exploring ML methods to predict bus arrival times and their uncertainty $[18,526]$. Often freight is packaged so that it can switch between different modes of transport easily. Such intermodal transportation relies on low-carbon modes such as rail and water for part of the journey [407]. ML can contribute by improving predictions of the estimated time of arrival (for example, of freight trains [56]) or the weight or volume of expected freight (for example, for roll-on/roll-off transport-often abbreviated as Ro-Ro [560]). Intelligent transport systems of different modes could be combined and enable more efficient multimodal freight transportation [407].

Some modes with high GHG emissions, such as trucks, can be particularly cost-competitive in regions with lax enforcement of regulation, as they can benefit from overloading and not obeying labor or safety rules [407]. ML can assist public institutions with enforcing their regulations. For example, image recognition can help law enforcement detect overloading of trucks [888]. 


\subsection{Discussion}

Decarbonizing transport is essential to a low-carbon society, and there are numerous applications where ML can make an impact. This is because transportation causes a large share of GHG emissions, but reducing them has been slow and complex. Solutions are likely very technical, are highly dependent on existing infrastructure, and require detailed understanding of passengers' and freight companies' behavior. ML can help decarbonize transportation by providing data, gaining knowledge from data, planning, and automation. Moreover, ML is fundamental to shared mobility, AVs, EVs, and smart public transit, which, with the right incentives, can be used to enable significant reductions in GHG emissions.

\section{BUILDINGS \& CITIES}

BY NIKOLA MILOJEVIC-DUPONT \& LYNN H. KAACK

Buildings offer some of the lowest-hanging fruit when it comes to reducing GHG emissions. While the energy consumed in buildings is responsible for a quarter of global energy-related emissions [372], a combination of easy-to-implement fixes and state-of-the-art strategies ${ }^{16}$ could reduce emissions for existing buildings by up to $90 \%$ [802]. It is possible today for buildings to consume almost no energy [595]. ${ }^{17}$ Many of these energy efficiency measures actually result in overall cost savings [754] and simultaneously yield other benefits, such as cleaner air for occupants. This potential can be achieved while maintaining the services that buildings provide-and even while extending them to more people, as climate change will necessitate. For example, with the changing climate, more people will need access to air conditioning in regions where deadly heat waves will become common [553, 554].

Two major challenges are heterogeneity and inertia. Buildings vary according to age, construction, usage, and ownership, so optimal strategies vary widely depending on the context. For instance, buildings with access to cheap, low-carbon electricity may have less need for expensive features such as intelligent light bulbs. Buildings also have very long lifespans; thus, it is necessary both to create new, energy-efficient buildings, and to retrofit old buildings to be as efficient as possible [150]. Urban planning and public policy can play a major role in reducing emissions by providing infrastructure, financial incentives, or energy standards for buildings [536]. ${ }^{18}$

ML provides critical tools for supporting both building managers and policymakers in their efforts to reduce GHG emissions (Figure 3). At the level of building management, ML can help select strategies that are tailored to individual buildings, and can also contribute to implementing those strategies via smart control systems (Section 4.1). At the level of urban planning, ML can be used to gather and make sense of data to inform policymakers (Section 4.2). Finally, we consider how ML can help cities as a whole to transition to low-carbon futures (Section 4.3).

\subsection{Optimizing Buildings}

In designing new buildings and improving existing ones, there are numerous technologies that can reduce GHG emissions, often saving money in the process [276, 499, 595, 754, 802]. ML can accelerate these strategies by (i) modeling data on energy consumption and (ii) optimizing energy use (in smart buildings).

\footnotetext{
${ }^{16}$ The IPCC classifies mitigation actions in buildings into four categories: carbon efficiency (switching to low-carbon fuels or to natural refrigerants); energy efficiency (reducing energy waste through insulation, efficient appliances, better heating and ventilation, or other similar measures); system and infrastructure efficiency (e.g., passive house standards, urban planning, and district cooling and heating); and service demand reduction (behavioral and lifestyle changes) [499].

${ }^{17}$ There are even high-rise buildings, e.g., the Tower Raiffeisen-Holding NÖ-Vienna office, or large university buildings, e.g., the Technical University also in Vienna, that achieve such performance.

${ }^{18}$ For example, see the case of New York City, which mandated that building owners collectively reduce their emissions by 40\% by 2040: https://www.nytimes.com/2019/04/17/nyregion/nyc-energy-laws.html.
} 


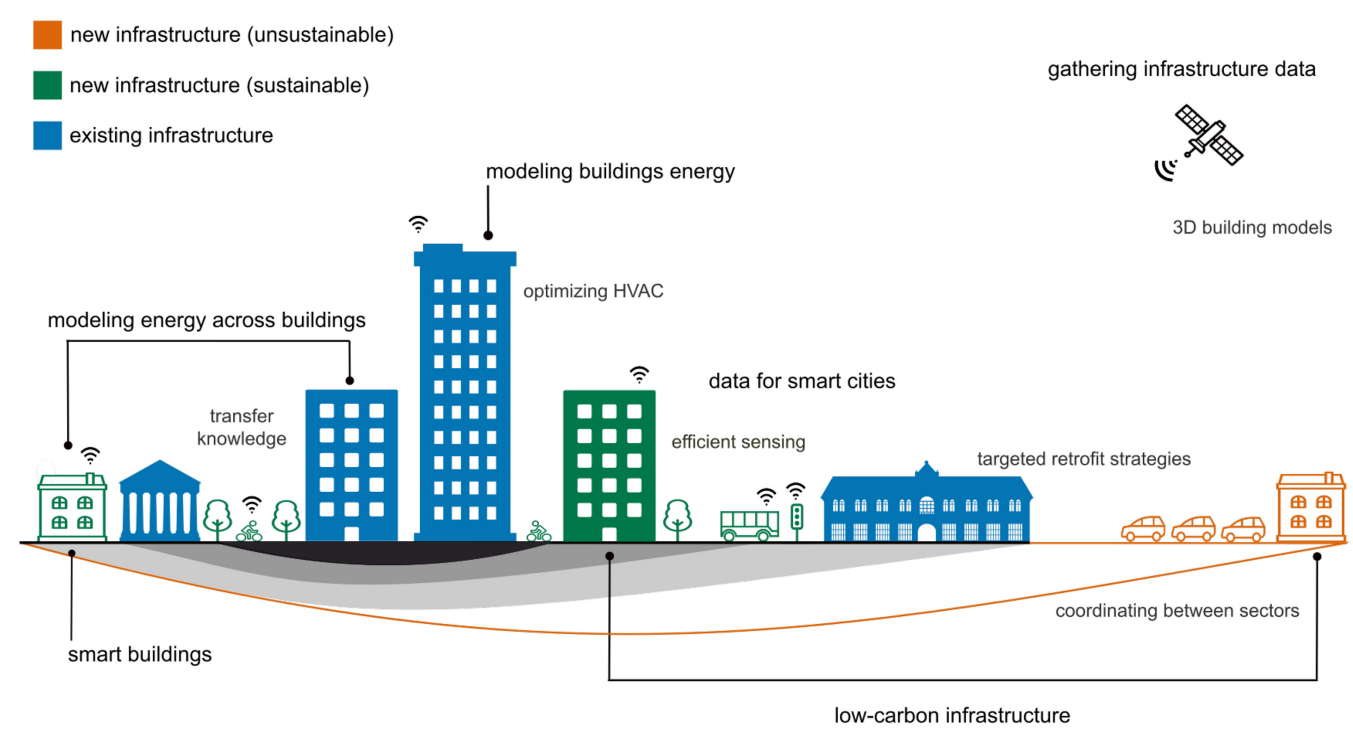

Fig. 3. Selected opportunities to reduce GHG emissions from buildings and cities using $\mathrm{ML}$, as described in Section 4.

\section{Modeling building energy.}

An essential step towards energy efficiency is making sense of the increasing amounts of data produced by meters and home energy monitors (see for example [723]). This can take the form of energy demand forecasts for specific buildings, which are useful for power companies (Section 2.1.1) and in evaluating building design and operation strategies [20]. Traditionally, energy demand forecasts are based on models of the physical structure of a building that are essentially massive thermodynamics computations. ML has the potential to speed up these computations greatly, either by ignoring physical knowledge of the building entirely [452, 617], by incorporating it into the computation [195], or by learning to approximate the physical model to reduce the need for expensive simulation (surrogate models) [274]. Learning how to transfer the knowledge gained from modeling one building to another can make it easier to render precise estimations of more buildings. For instance, Mocanu et al. [544] modeled building load profiles with RL and deep belief networks using data on commercial and residential buildings; they then used approximate RL and transfer learning to make predictions about new buildings, enabling the transfer of knowledge from commercial to residential buildings, and from gas- to power-heated buildings.

Within a single building, understanding which appliances drive energy use (energy disaggregation) is crucial for targeting efficiency measures, and can motivate behavioral changes. Promising ML approaches to this problem include hidden Markov models [445], sparse coding algorithms for structured prediction [443], harmonic analysis that picks out the "signatures" of individual appliances [750], and deep neural networks [423].

To verify the success or failure of energy efficiency interventions, statistical ML offers methods for causal inference. For example, Burlig et al. [102] used Lasso regression on hourly electricity consumption data from schools in California to find that energy efficiency interventions fall short of the expected savings. Such problems could represent a useful application of deep learning methods for counterfactual prediction [332]. 
Smart buildings.

High Leverage

Intelligent control systems in buildings can decrease their carbon footprint both by reducing the energy consumed and by providing means to integrate lower-carbon sources into the electricity mix [277]. Specifically, ML can reduce energy usage by allowing devices and systems to adapt to usage patterns. Further, buildings can respond to signals from the electricity grid, providing flexibility to the grid operator and lowering costs to the consumer (Section 2.1.1).

Many critical systems inside buildings can be made radically more efficient. While this is also true for various appliances such as refrigerators and lightbulbs, we focus on the example of heating, ventilation, and air conditioning (HVAC) systems, both because they are notoriously inefficient and because they account for more than half of the energy consumed in buildings [499]. There are several promising ways to enhance HVAC operating performance, each providing substantial opportunities for using ML: forecasting what temperatures are needed throughout the system, better control to achieve those temperatures, and fault detection. Forecasting temperatures, as with modeling energy use in buildings, has traditionally been performed using detailed physical models of the system involved; however, ML approaches such as deep belief networks can potentially increase accuracy with less computational expense $[4,255]$ (see also Section 5.3). For control, Kazmi et al. [417] used deep RL to achieve a scalable $20 \%$ reduction of energy while requiring only three sensors: air temperature, water temperature, and energy use (see also Section 5.3 for similarly substantial gains in datacenter cooling). Finally, ML can automate building diagnostics and maintenance through fault-detection. For example, the energy efficiency of cooling systems can degrade if refrigerant levels are low [435]; ML approaches are well-suited to detect faults in these systems. Wang et al. [829] treated HVAC fault-detection as a one-class classification problem, using only temperature readings for their predictions. Deep autoencoders can be used to simplify information about machine operation so that deep neural networks can then more easily predict multiple kinds of faults [387].

Many systems within buildings-such as lights and heating-can also adjust how they operate based on whether a building or room is occupied, thereby improving both occupant comfort and energy use [614]. ML can help these systems dynamically adapt to changes in occupancy patterns [669]. Moreover, occupancy detection itself represents an opportunity for ML algorithms, ranging from decision trees [193, 882] to deep neural networks [896] that take input from occupancy sensors [193], WiFi signals [896, 897], or appliance power consumption data [882]. Energy game-theoretic frameworks can also incentivize occupants to actively minimize their energy demand, and ML can help here to develop tailored incentives based on different energy usage behaviors [159].

In Section 2.1.1, we discussed how using variable low-carbon energy can mean that the supply and price of electricity vary over time. Thus, energy flexibility in buildings is increasingly useful to schedule consumption when supply is high [683]. For this, automated demand-side response [357] can respond to electricity prices, smart meter signals, or learned user preferences [393]. Edge computing can be used to process data from distributed sensors and other Internet of Things devices, and deep RL can then use this data to efficiently schedule energy use [489], at the level of single or multiple buildings, or at the microgrid and grid level [631].

While smart building technologies have the capability to significantly increase efficiency, we should note that there are potential drawbacks [346]. First, smart building devices and connection networks, like wireless sensor networks, consume energy themselves. Deep neural networks can be used to monitor and optimize such operations [39]. Second, rebound effects are likely to happen in certain cases [45], leading to additional building energy consumption typically ranging between 10 and 20\% [671]. Third, if control systems optimize for costs, interventions 
do not necessarily translate into energy efficiency measures or GHG reductions. Therefore, public policies are needed to mandate, support and complement the actions of individual building managers [499]. Another concern in the case of widespread adoption of smart meters is the impact on mineral use and embodied energy use arising from their production [736]. Finally, smart home applications present security and privacy risks [144] that require adequate regulation.

\subsection{Urban Planning}

For many impactful mitigation strategies-such as district heating and cooling, neighborhood planning, and large-scale retrofitting of existing buildings-coordination at the district and city level is essential. Policymakers use instruments such as building codes, retrofitting subsidies, investments in public utilities, and public-private partnerships in order to reduce GHG emissions without compromising equity. Where energy-use data on individual buildings exist, ML can be used to derive higher-level patterns. However, many regions of the world have almost no energy consumption data, which can make it difficult to design targeted mitigation strategies. ML is uniquely capable of predicting energy consumption and GHG mitigation potential at scale from other types of available data, thereby guiding policy design [536].

\section{Modeling energy use across buildings.}

Urban Building Energy Models (UBEMs) provide simplified information on the energy use of all buildings across a city. These are different from individual building models, which model energy use of only specific buildings, but with finer details and temporal granularity (Section 4.1). While UBEMs have yet to be adopted at scale, they are expected to become fundamental for enabling localized action by city planners [677]. UBEMs can for example be used for planning and operating district heating and cooling, where a central plant supplies many households in a district. In turn, district heating and cooling reduces HVAC energy consumption and can provide flexible load [808], but it needs large amounts of data at the district level for implementation and operation.

UBEMs include features such as the location, geometries, and various other attributes of interest like building footprint, usage, material, roof type, immediate surroundings, and the like. ML can be used to held predict energy consumption from such features. For example, Kolter and Ferreira used Gaussian process regression to predict energy use from features such as property class or the presence of central air conditioning [444]. Based on energy data disclosed by residents of New York City, Kontokosta and colleagues used various ML methods to predict the energy use of the city's 1.1 million buildings [448], analyzed the effect of energy disclosure on the demand [610], and developed a system for ranking buildings based on energy efficiency [611]. Zhang et al. [879] matched residential energy consumption survey data with public use microdata samples to estimate residential energy consumption at the neighborhood level. Using five commonly accessible features of buildings and climate, Robinson et al. predict commercial building energy use across large American cities [689].

Beyond energy prediction, buildings' features can be used by $\mathrm{ML}$ algorithms to pinpoint which buildings have the highest retrofit potential. Simple building characteristics and surrounding environmental factors-both potentially available at scale-can be used [83, 432].

There have also been attempts to upscale individual-building energy models to the district scale. Using deep neural networks for hybrid ML-physical modelling, Nutkiewicz et al. provided precise energy demand forecasts that account for inter-building energy dynamics and urban microclimate factors for all buildings on a campus [589].

Gathering infrastructure data.

High Leverage

Specifics about building infrastructure can often be predicted using ML techniques. Remote 
sensing is key to inferring infrastructure data $[79,224,339,496,535,868]$ as satellite data ${ }^{19}$ present a source of information that is globally available and largely consistent worldwide. For example, using remote sensing data, Geiß et al. [273] clustered buildings into types to assess the potential of district heat in a German town.

The resolution of infrastructure data ranges from coarse localization of all buildings at the global scale [224], to precise 3D reconstruction of a neighborhood [79]. It is possible to produce a global map of human settlement footprints at meter-level resolution from satellite radar images [224] For this, Esch et al. used highly automated learners, which make classification at such scale possible by retraining locally. Segmentation of high-resolution satellite images can now generate exact building footprints at a national scale [535]. Energy-relevant building attributes, such as the presence of photovoltaic panels, can also be retrieved from these images [868] (see Section 2.1.1). To generate 3D models, LiDAR data are often used to retrieve heights or classify buildings at city scale $[339,496]$, but its collection is expensive. Recent research showed that heights can be predicted even without such elevation data, as demonstrated by [77, 537], who predicted these from real estate records, census data and features characterizing the neighborhood of each building. Studies, which for now are small scale, aim for complete 3D reconstruction with class labels for different components of buildings [79].

\subsection{The Future of Cities}

Since most of the resources of the world are ultimately channeled into cities, municipal governments have a unique opportunity to mitigate climate change. City governments regulate (and sometimes operate) transportation, buildings, and economic activity. They handle such diverse issues as energy, water, waste, crime, health, and noise. Recently, data and ML have become more common for improving efficiency in such areas, giving rise to the notion of smart city. While the phrase smart city encompasses a wide array of technologies [579], here we discuss only applications that are relevant to reducing GHG emissions.

\section{Data for smart cities.}

High Leverage

Increasingly, important aspects of city life come with digital information that can make the city function in a more coordinated way. Habibzadeh et al. [319] differentiate between hard-sensing, i.e., fixed-location-dedicated sensors like traffic cameras, and soft-sensing, for example, from mobile devices. Hard sensing is the primary data collection paradigm in many smart city applications, as it is adapted to precisely meet the application requirements. However, there is a growing volume of data coming from soft sensing, due to the widespread adoption of personal devices like smartphones that can provide movement data and geotagged pictures. ${ }^{20}$ Urban computing [886] is an emerging field looking at data analytics in urban spaces, and aiming to yield insights for data-driven policies. For example, clustering anonymized credit card payments makes it possible to model different communities and lifestyles-of which the sustainability can be assessed [181] Jiang et al. provides a review of urban computing from mobile phone traces [391]. ${ }^{21}$ Relevant information on the urban space can also be learned from social media activity, e.g., on Twitter, as reviewed in $[367,703]$. There are, however, numerous challenges in making sense of this wealth of data (see [558]), and privacy considerations are of paramount importance when collecting or working with many of these data sources.

\footnotetext{
${ }^{19}$ See [893] for a review of different sources of data and deep learning methods for processing them.

${ }^{20}$ Note that management of any such private data, even if they are anonymized, poses challenges [153].

${ }^{21}$ See https://www.microsoft.com/en-us/research/project/urban-computing/ for more applications of urban computing.
} 
First, cities need to obtain relevant data on activities that directly or indirectly consume energy, and such data are often proprietary. To obtain these data, the city of Los Angeles now requires all mobility as a service providers, i.e., vehicle-sharing companies, to use an open-source application programming interface. Data such as location, use, and condition of all those vehicles, which can be useful in guiding regulation, are thus transmitted to the city [134]. ML can also distill information on urban issues related to climate change through web-scraping and text-mining, e.g., [775]. As discussed above (Section 4.2), ML can also be used to infer infrastructure data.

Second, smart city applications must transmit high volumes of data in real-time. ML is key to preprocessing large amounts of data in large sensor networks, allowing only what is relevant to be transmitted, instead of all the raw data that is being collected [480, 672, 804]. Similar techniques also help to reduce the amount of energy consumed during transmission itself [563].

Third, urban policy-making based on intelligent infrastructure faces major challenges with data management [286]. Smart cities require the integration of multiple large and heterogeneous sources of data, for which ML can be a valuable tool, which includes data matching $[89,190]$, data fusion [885], and ensemble learning [451].

\section{Low-emissions infrastructure.}

When smart city projects are properly integrated into urban planning, they can make cities more sustainable and foster low-carbon lifestyles (see [563, 602, 853] for extensive reviews on this topic). Different types of infrastructure interact, meaning that planning strategies should be coordinated to achieve mitigation goals. For instance, urban sprawl influences the energy use from transport, as wider cities tend to be more car-oriented [151, 189, 228]. ML-based analysis has shown that the development of efficient public transportation is dependent on both the extent of urban sprawl and the local development around transportation hubs [547, 737].

Cities can reduce GHG emissions by coordinating between infrastructure sectors and better adapting services to the needs of the inhabitants. ML and AI can help, for example, to coordinate district heating and cooling networks, solar power generation, and charging stations for EVs and bikes [602], and can improve public lighting systems by regulating light intensity based on historical patterns of foot traffic [169]. Due to inherent variability in energy demand and supply, there is a need for uncertainty estimation, e.g., using Markov chain Monte Carlo methods or Gaussian processes [602].

Currently, most smart city projects for urban climate change mitigation are implemented in wealthier regions such as the United States, China, and the European Union. ${ }^{22}$ The literature on city-scale mitigation strategies is also strongly biased towards the Global North [466], while key mitigation challenges are expected to arise from the Global South [570]. Infrastructure models described in Section 4.2 could be used to plan low-carbon neighborhoods without relying on advanced smart city technologies. To transfer strategies across cities, it is possible to cluster similar cities based on climate-relevant dimensions [326, 494]. Creutzig et al. [151] related the energy use of 300 cities worldwide to historical structural factors such as fuel taxes (which have a strong impact on urban sprawl). Other relevant applications include groupings of transportation systems [326] using a latent class choice model, or of street networks [494] to identify common patterns in urban development using hierarchical clustering.

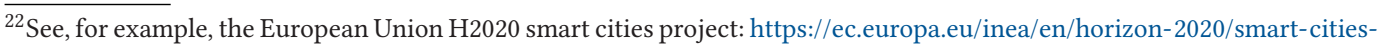
communities.
} 


\subsection{Discussion}

We have shown many different ways that ML can help to reduce GHG emissions from buildings and cities. A central challenge in this sector is the availability of high-quality data for training the algorithms, which rarely go beyond main cities or represent the full spectrum of building types. Techniques for obtaining these data, however, can themselves be an important application for ML (e.g., via computer vision algorithms to parse satellite imagery). Realizing the potential of data-driven urban infrastructure can advance mitigation goals while improving the well-being of citizens $[50,150,802]$.

\section{INDUSTRY}

BY ANNA WALDMAN-BROWN

Industrial production, logistics, and building materials are leading causes of difficult-to-eliminate GHG emissions [162]. Fortunately for ML researchers, the global industrial sector spends billions of dollars annually gathering data on factories and supply chains [310]-aided by improvements in the cost and accessibility of sensors and other data-gathering mechanisms (such as QR codes and image recognition). The availability of large quantities of data, combined with affordable cloudbased storage and computing, indicates that industry may be an excellent place for ML to make a positive climate impact.

ML demonstrates considerable potential for reducing industrial GHG emissions under the following circumstances:

- When there is enough accessible, high-quality data around specific processes or transport routes.

- When firms have an incentive to share their proprietary data and/or algorithms with researchers and other firms.

- When aspects of production or shipping can be readily fine-tuned or adjusted, and there are clear objective functions.

- When firms' incentives align with reducing emissions (for example, through efficiency gains, regulatory compliance, or high GHG prices).

In particular, ML can potentially reduce global emissions (Figure 4) by helping to streamline supply chains, improve production quality, predict machine breakdowns, optimize heating and cooling systems, and prioritize the use of clean electricity over fossil fuels [74, 227, 416, 880]. However, it is worth noting that greater efficiency may increase the production of goods and thus GHG emissions (via rebound effects) unless industrial actors have sufficient incentives to reduce overall emissions [748].

\subsection{Optimizing Supply Chains}

In 2006, at least two Scottish seafood firms flew hundreds of metric tons of shrimp from Scotland to China and Thailand for peeling, then back to Scotland for sale-because they could save on labor costs [147]. This indicates the complexity of today's globalized supply chains, i.e., the organizational processes and shipping networks that are required to bring a product from producer to final consumer. ML can help reduce emissions in supply chains by intelligently predicting supply and demand, identifying lower-carbon products, and optimizing shipping routes. (For details on shipping and delivery optimization, see Section 3.) However, for many of these applications to reduce emissions, firms' financial incentives must also align with climate change mitigation through carbon pricing or other policy mechanisms.

Reducing overproduction.

Uncertain Impact The production, shipment, and climate-controlled warehousing of excess products is a major 


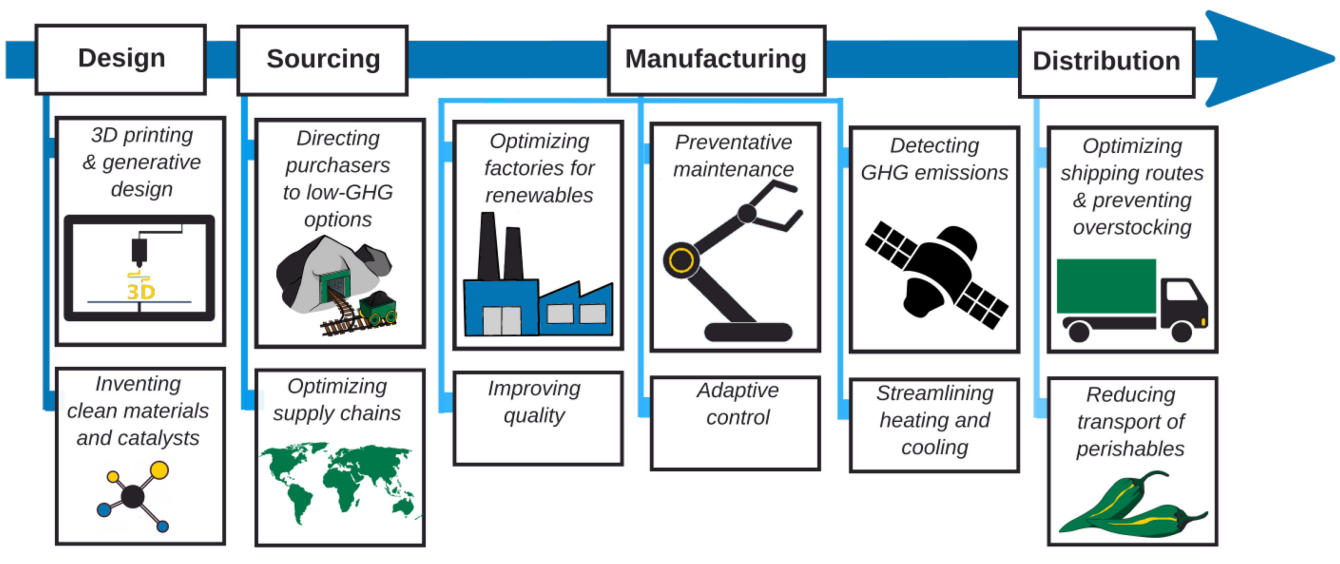

Fig. 4. Selected opportunities to reduce GHG emissions in industry using ML, as described in Section 5.

source of industrial GHG emissions, particularly for time-dependent goods such as perishable food or retail goods that quickly fall out of fashion [828]. Global excess inventory in 2011 amounted to about $\$ 8$ trillion worth of goods, according to the Council of Supply Chain Management Professionals [847]. This excess may be in part due to mis-estimation of demand, as the same organization noted that corporate sales estimates diverged from actual sales by an average of $40 \%$ [847]. ML may be able to mitigate these issues of overproducing and/or overstocking goods by improving demand forecasting [12, 797]. For example, the clothing industry sells an average of only $60 \%$ of its wares at full price, but some brands can sell up to $85 \%$ due to just-in-time manufacturing and clever intelligence networks [294]. As online shopping and just-in-time manufacturing become more prevalent and websites offer more product types than physical storefronts, better demand forecasts will be needed on a regional level to efficiently distribute inventory without letting unwanted goods travel long distances only to languish in warehouses [685]. Nonetheless, negative side effects can be significant depending on the type of product and regional characteristics; just-in-time manufacturing and online shopping are often responsible for enabling product fads with shorter lifespans, in addition to smaller and faster shipments of goods (mostly by road) that lack the energy efficiency of freight aggregation and slower shipping methods such as rail $[685,799]$.

\section{Recommender systems.}

Recommender systems can potentially direct consumers and purchasing firms toward climatefriendly options, as long as one can obtain information about GHG emissions throughout the entire life-cycle of some product. The challenge here lies in hunting down usable data on every relevant material and production process from metal ore extraction through production, shipping, and eventual use and disposal of a product $[336,674]$. One must also convince companies to share proprietary data to help other firms learn from best practices. If these datasets can be acquired, ML algorithms could hypothetically assist in identifying the cleanest options.

\section{Reducing food waste.}

High Leverage

Globally, society loses or wastes 1.3 billion metric tons of food each year, which translates to onethird of all food produced for human consumption [316]. In developing countries, $40 \%$ of food waste occurs between harvest and processing or retail, while over $40 \%$ of food waste in industrialized nations occurs at the end of supply chains, in retail outlets, restaurants, and consumers' homes 
[316]. ML can help reduce food waste by optimizing delivery routes and improving demand forecasting at the point of sale (see Section 5.1), as well as improving refrigeration systems [532] (see Section 5.3). ML can also potentially assist with other issues related to food waste, such as helping develop sensors to identify when produce is about to spoil, so it can be sold faster or removed from a storage crate before it ruins the rest of the shipment [256].

\subsection{Improving Materials}

\section{Climate-friendly construction.}

High Leverage Long-term

Cement and steel production together account for over $10 \%$ of all global GHG emissions [243]; the cement industry alone emits more GHGs than every country except the USA and China [477]. ML can help minimize these emissions by reducing the need for carbon-intensive materials, by transforming industrial processes to run on low-carbon energy, and even by redesigning the chemistry of structural materials. To reduce the use of cement and steel, researchers have combined ML with generative design to develop structural products that require less raw material, thus reducing the resulting GHG emissions [416]. Novel manufacturing techniques such as 3D printing allow for the production of unusual shapes that use less material but may be impossible to produce through traditional metal-casting or poured concrete; ML and finite element modeling have been used to simulate the physical processes of 3D printing in order to improve the quality of finished products [62].

Assuming future advances in materials science, ML research could potentially draw upon open databases such as the Materials Project [380] and the UCI Machine Learning Repository [271] to invent new, climate-friendly materials [830]. Using semi-supervised generative models and concrete compression data, for example, Ge et al. proposed novel, low-emission concrete formulas that could satisfy desired structural characteristics [271].

Climate-friendly chemicals.

High Leverage Long-term

Researchers are also experimenting with supervised learning and thermal imaging systems to rapidly identify promising catalysts and chemical reactions $[140,686]$, as described in Section 2.1.1. Firms are unlikely to adopt new materials or change existing practices without financial incentives, so widespread adoption might require subsidies for low-carbon alternatives or penalties for high GHG emissions.

Ammonia production for fertilizer use relies upon natural gas to heat up and catalyze the reaction, and accounts for around $2 \%$ of global energy consumption [551]. To develop cleaner ammonia, chemists may be able to invent electrochemical strategies for lower-temperature ammonia production [551, 849]. Given the potential of ML for predicting chemical reactions [140], ML may also be able to help with the discovery of new materials for electrocatalysts and/or proton conductors to facilitate ammonia production.

\subsection{Production and Energy}

ML can potentially assist in reducing overall electricity consumption, streamlining factories' HVAC systems, and developing models for electrifying industrial processes so they can be run on low-carbon energy instead of coal, oil, or natural gas [305]. Again, the higher the incentives for reducing carbon emissions, the more likely that firms will optimize for low-carbon energy use. New factory equipment can be very expensive to purchase and set up, so firms' cost-benefit calculations may dissuade them from retrofitting existing factories to run using low-carbon electricity or to save a few kilowatts $[288,633,778]$. Given the heterogeneity across industrial sectors and the secrecy of industrial data, firms will also need to tailor the requisite sensors and data analysis systems to their individual processes. ML will become a much more viable option for industry 
when factory workers can identify, develop, implement, and monitor their own solutions internally instead of relying upon outside experts [337]. The ML community can assist by building accessible, customizable industry tools (i.e., "low-code" or "no-code" user interfaces) tailored for people without a strong background in data science.

Adaptive control.

High Leverage

On the production side, ML can potentially improve the efficiency of HVAC systems and other industrial control mechanisms-if given necessary data about all relevant processes. To reduce GHG emissions from HVAC systems, researchers have suggested combining optimization-based control algorithms with ML techniques such as image recognition, regression trees, and time delay neural networks [5, 202] (see also 4.1). DeepMind has used RL to optimize cooling centers for Google's internal servers by predicting and optimizing the power usage effectiveness, thus lowering HFC emissions and reducing cooling costs [227, 268]. Deep neural networks could also be used for adaptive control in a variety of industrial networking applications [8], enabling energy savings through self-learning about devices' surroundings.

\section{Predictive maintenance.}

ML could also contribute to predictive maintenance (see also Sections 2.2 and 9.2) by more accurately modelling the wear and tear of machinery that is currently in use, and interpretable ML could assist factory owners in developing a better understanding of how best to minimize GHG emissions for specific equipment and processes. For example, creating a digital twin model of some industrial equipment or process could enable a manufacturer to virtually experiment with a new piece of code before uploading it to the factory floor, and to test out scenarios for lower GHG emissions without slowing down production [290,777]. Digital twins can also reduce production waste by identifying broken or about-to-break machines before the actual factory equipment starts producing damaged products. Industrial systems can employ similar models to predict which pipes are liable to spring leaks, thus minimizing the direct release of GHGs such as HFCs and natural gas.

Using cleaner electricity.

High Leverage

ML may be particularly useful for enabling more flexible operation of industrial electrical loads, through optimizing a firm's demand response to electricity prices (see also Section 2). Such optimization can contribute to cutting GHG emissions as long as firms have a financial incentive to optimize for minimal emissions, maximal low-carbon energy, or minimum overall power usage. Demand response optimization algorithms can help firms adjust the timing of energy-intensive processes such as cement crushing [880] and powder-coating [43] to take advantage of electricity price fluctuations, although published work on the topic has to date used relatively little ML. Online algorithms for optimizing demand response can reduce overall power usage for computer servers by dynamically shifting the internet traffic load of data providers to underutilized servers, although most of this research, again, has focused on minimizing costs rather than GHG emissions $[99,352]$. Berral et al. proposed a framework that demonstrates how such optimization algorithms might be combined with RL, digitized controls, and feedback systems to enable the autonomous control of industrial processes [74].

\subsection{Discussion}

Given the globalized nature of international trade and the urgency of climate change, decarbonizing the industrial sector is becoming a key priority for both policymakers and factory owners worldwide. Many companies are now writing decarbonization strategies in response to increasing 
pressure from governments, financial institutions, and stockholders (see, e.g., [620, 793]). These strategies are evolving rapidly, using ML alongside many approaches not covered here.

As we have seen, there are a number of highly impactful applications where ML can help reduce GHG emissions in industry, with several caveats. First, incentives for cleaner production and distribution are not always aligned with reduced costs, though policies can play a role in aligning these incentives. Second, despite the proliferation of industrial data, much of the information is proprietary, low-quality, or very specific to individual machines or processes; practitioners estimate that $60 \%-70 \%$ of collected industrial data goes unused [138, 310]. Before investing in extensive ML research, researchers should be sure that they will be able to eventually access and clean any data needed for their algorithms. Finally, misjudgments can be very costly for manufacturers and retailers, leading most managers to adopt risk-averse strategies towards relatively untested technologies such as ML [337]. For this reason, ML algorithms that determine industrial activities should be robust enough to guarantee both performance and safety, along with providing both interpretable and reproducible results [338, 709].

\section{FARMS \& FORESTS}

BY ALEXANDRE LACOSTE

Plants, microbes, and other organisms have been drawing $\mathrm{CO}_{2}$ from the atmosphere for millions of years. Most of this carbon is continually broken down and recirculated through the carbon cycle, and some is stored deep underground, e.g., as fossil fuels, but a large amount of carbon is sequestered in the biomass of trees, peat bogs, and soil. Our current economy encourages practices that are freeing much of this sequestered carbon through deforestation and unsustainable agriculture. On top of these effects, cattle and rice farming generate methane, a GHG far more potent than $\mathrm{CO}_{2}$ itself. Overall, land use by humans is estimated to be responsible for about a quarter of global GHG emissions [370] (and this may be an underestimate [511]). In addition to this direct release of carbon through human actions, the permafrost is now melting, peat bogs are drying, and forest fires are becoming more frequent as a consequence of climate change itself - all of which release yet more carbon [573].

The large scale of this problem allows for a similar scale of positive impact. According to one estimate [335], about a third of GHG emissions reductions could come from better land management and agriculture. ML can play an important role in some of these areas. Precision agriculture could reduce carbon release from the soil and improve crop yield, which in turn could reduce the need for deforestation. Satellite images make it possible to estimate the amount of carbon sequestered in a given area of land, as well as track GHG emissions from it. ML can help monitor the health of forests and peatlands, predict the risk of fire, and contribute to sustainable forestry (Figure 5). These areas represent highly impactful applications, in particular, of sophisticated computer vision tools, though care must be taken in some cases to ensure that ML tools are used in ways aligned with decarbonization.

\subsection{Remote Sensing of Emissions}

High Leverage

Having real-time maps of GHGs could help us quantify emissions from agriculture and forestry practices, which remain relatively uncertain [371], as well as monitor emissions from other sectors (Section 2.2).

Such information would be valuable in guiding regulations or incentives that could lead to better land use practices. For example, data on emissions make it possible to set effective targets, and pinpointing the sources of emissions makes it possible to enforce regulations.

While GHGs are invisible to our eyes, they must by definition interact with sunlight. This means that we can observe these compounds with hyperspectral cameras [428, 711]. These 


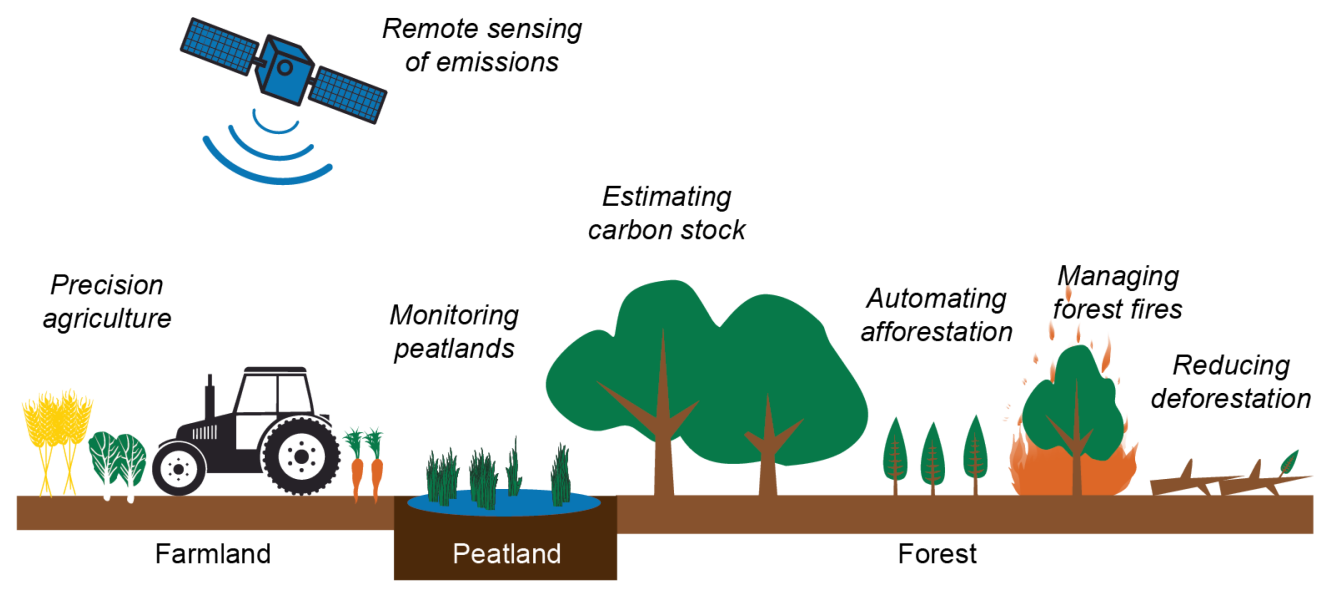

Fig. 5. Selected opportunities to reduce GHG emissions from land use using ML, as described in Section 6.

cameras can record up to several hundred different wavelengths (instead of simply red, green, and blue [RGB]), providing information on the interaction between light and individual chemicals. Many satellites are equipped with such cameras and can perform, to some extent, estimations of $\mathrm{CO}_{2}, \mathrm{CH}_{4}$ (methane), $\mathrm{H}_{2} \mathrm{O}$, and $\mathrm{N}_{2} \mathrm{O}$ (nitrous oxide) emissions [73, 378]. While extremely useful for studying climate change, most of these satellites have very coarse spatial resolution and large temporal and spatial gaps, making them unsuitable for precise tracking of emissions. Standard satellite imagery provides RGB images with much higher resolution, which could be used in an ML algorithm to fill the gaps in hyperspectral data and obtain more precise information about emissions. ${ }^{23}$ Some preliminary work [378] has studied this possibility, but this remains largely an open problem with high potential impact.

\subsection{Precision Agriculture}

High Leverage Uncertain Impact

Crop production is a significant source of GHG emissions. This might come as a surprise, since plants take up $\mathrm{CO}_{2}$ from the air. However, modern industrial crop production involves more than just growing plants. First, the land is generally stripped of existing vegetation, releasing carbon sequestered there. Second, the process of tilling exposes topsoil to the air, thereby releasing carbon that had been bound in soil aggregates and disrupting organisms in the soil that contribute to sequestration. Finally, because such farming practices strip soil of nutrients, nitrogen-based fertilizers must be added back to the system. Synthesizing these fertilizers consumes massive amounts of energy, about $2 \%$ of global energy consumption [551] (see Section 5.2). Moreover, while some of this nitrogen is absorbed by plants or retained in the soil, some is converted to nitrous oxide, ${ }^{24}$ a GHG that is about 300 times more potent than $\mathrm{CO}_{2}$.

Such industrial agriculture approaches are ultimately based on making farmland more uniform and predictable. This allows it to be managed at scale using basic automation tools like tractors, but can be both more destructive and less productive than approaches that work with the natural

\footnotetext{
${ }^{23}$ Satellites with higher resolution hyperspectral cameras are beginning to deploy, including GHGSat satellites in already orbit and plans by Carbon Mapper, Bluefield Technologies, and the Environmental Defense Fund to launch satellites in coming years $[81,113,534]$. Even once this technology comes online, ML will remain useful to cover gaps and to estimate emissions of other GHGs.

${ }^{24}$ Some fertilizer additionally often ends up in waterways, which can contaminate drinking water and induce blooms of toxic algae [687].
} 
heterogeneity of land and crops. Increasingly, there is demand for sophisticated tools which would allow farmers to work at scale, but adapt to what the land needs. This approach is often known as "precision agriculture."

Smarter robotic tools can help enable precision agriculture. Robots are under development, for example, with the ability to perform mechanical weeding, targeted pesticide application, and vacuuming of pests [766], as well as the collection of large datasets for continual improvement [68]. Many corporate players now exist in the space of ML-aided robotics for precision agriculture $[80,212,742,788]$. There remains significant room for development, since many tasks remain challenging for robots, and furthermore there are a large number of specific tasks and agricultural settings to consider.

There are many additional ways in which ML can contribute to precision agriculture. Intelligent irrigation systems can save large amounts of water while reducing pests that thrive under excessive moisture [335]. ML can also help in disease detection, weed detection, and soil sensing [482, 699, 821]. ML can guide crop yield prediction [866] and even macroeconomic models that help farmers predict crop demand and decide what to plant at the beginning of the season [503]. These problems often have minimal hardware requirements, as devices such as Unmanned Aerial Vehicles (UAVs) with hyperspectral cameras can be used for all of these tasks.

Globally, agriculture constitutes a $\$ 2.4$ trillion industry [785], and there is already a significant economic incentive to increase efficiency. However, efficiency gains do not necessarily translate into reduced GHG emissions (e.g., via rebound effects increasing consumption of particularly emissions-intensive products). Moreover, significantly reducing emissions may require a shift in agricultural paradigms, for example, widespread adoption of regenerative agriculture, silvopasture, and tree intercropping [335]. ML tools for policymakers and agronomists [126] could potentially encourage climate-positive action: for example, remote sensing with UAVs and satellites could perform methane detection and carbon stock estimation, which could be used to incentivize farmers to sequester more carbon and reduce emissions.

\subsection{Monitoring Peatlands}

High Leverage

Peatlands (a type of wetland ecosystem) cover only 3\% of the Earth's land area, yet hold twice the total carbon in all the world's forests, making peat the largest source of sequestered carbon on Earth [612]. When peat dries, however, it releases carbon through decomposition and also becomes susceptible to fire [245, 612]. A single peat fire in Indonesia in 1997 is reported to have released emissions comparable to $20 \%-50 \%$ of global fossil fuel emissions during the same year [606].

Monitoring peatlands and protecting them from artificial drainage or droughts is essential to preserve the carbon sequestered in them [349, 400]. In [538], ML was applied to features extracted from remote sensing data to estimate the thickness of peat and assess the carbon stock of tropical peatlands. A central database for peatland-monitoring has been established, but considerable data gaps remain [121]. Advanced ML could potentially help develop precise monitoring tools at low cost, as well as predicting the risk of fire.

\subsection{Managing Forests}

Estimating carbon stock.

High Leverage

Modeling (and pricing) carbon stored in forests requires us to assess how much is being sequestered or released across the planet. Since most of a forest's carbon is stored in above-ground biomass [693], tree species and heights are a good indicator of the carbon stock.

The height of trees can be estimated fairly accurately with LiDAR devices mounted on UAVs, but this technology is not scalable and many areas are closed to UAVs. To address this challenge, ML 
can be used to predict the LiDAR's outcome from satellite imagery [48, 599, 693]. From there, the learned estimator can perform predictions at scale. Despite progress in this area, there is still significant room for improvement. For example, LiDAR data are often not equally distributed across regions or seasons. Hence domain adaptation and transfer learning techniques may help algorithms to generalize better.

Automating afforestation.

Long-term Uncertain Impact

Planting trees, also called afforestation, can be a means of sequestering $\mathrm{CO}_{2}$ over the long term. According to one estimate, up to 0.9 billion hectares of extra canopy cover could theoretically be added [60] globally. However, care must be taken when planting trees to ensure a positive impact. Afforestation that comes at the expense of farmland (or ecosystems such as peat bogs) could result in a net increase of GHG emissions. Moreover, planting trees without regard for local conditions and native species can reduce the climate impact of afforestation as well as negatively affecting biodiversity.

ML can be helpful in automating large-scale afforestation by locating appropriate planting sites, monitoring plant health, assessing weeds, and analyzing trends. For example, startups like Dendra Systems and Droneseed are developing UAVs that are capable of planting seed packets more quickly and cheaply than traditional methods [176, 204], while Restor uses ML to learn from past afforestation projects about effective strategies for ecosystem restoration [681].

Managing forest fires.

Besides their potential for harming people and property, forest fires release $\mathrm{CO}_{2}$ into the atmosphere (which in turn increases the rate of forest fires [839]). On the other hand, small forest fires are part of natural forest cycles. Preventing them causes biomass to accumulate on the ground and increases the chances of large fires, which can then burn all trees to the ground and erode top soil, resulting in high $\mathrm{CO}_{2}$ emissions, biodiversity loss, and a long recovery time [550]. Drought forecasting [682] is helpful in predicting regions that are more at risk, as is estimating the water content in the tree canopy [94]. In [266, 267], RL is used to predict the spatial progression of fire. This helps firefighters decide when to let a fire burn and when to stop it [355]. With good tools to evaluate regions that are more at risk, firefighters can perform controlled burns and cut select areas to prevent the progression of fires.

Reducing deforestation.

High Leverage

Only $17 \%$ of the world's forests are legally protected [506]. The rest are subject to deforestation, which contributes to approximately $10 \%$ of global GHG emissions [370] as vegetation is burned or decays. About $80 \%$ percent of global deforestation is the result of agriculture (clearing land for pasture or crop production), while other significant causes include mining, logging, and urban development [353].

Tools for tracking deforestation can provide valuable data for informing policymakers, as well as law enforcement in cases where deforestation may be conducted illegally. ML can be used with remote sensing imagery to pinpoint changes in forest cover [241, 329, 369], or proxies for deforestation such as smoke from fires set to clear vegetation [505], as well as to differentiate selective cutting from clearcutting $[342,487]$. ML can also be used with audio instead of visual data; one such project installs (old) smartphones powered by solar panels in the forest, which enables the detection of chainsaw sounds within a one-kilometer radius [657].

ML can also be used to help build incentive structures for sustainable forest management. Some companies are using ML-enabled tools to quantify the carbon impact of forestry decisions, enabling landowners to choose more beneficial actions as well as profit by selling carbon offsets [578, 603]. ML can also help in proving that tracts of forest are indeed being preserved (integrating data 
sources such as satellite imagery, UAV-based monitoring, and indigenous participatory mapping), thereby providing verification of carbon credits or other incentive structures for land custodians or owners [264, 501].

\subsection{Discussion}

Farms and forests make up a large portion of global GHG emissions, but reducing these emissions is challenging. The scope of the problem is highly globalized, but the necessary actions are highly localized. Many applications also involve a diversity of stakeholders. Agriculture, for example, involves a complex mix of large-scale farming interests, small-scale farmers, agricultural equipment manufacturers, and chemical companies. Each stakeholder has different interests, and each often has access to a different portion of the data that would be useful for impactful ML applications. Interfacing between these different stakeholders is a practical challenge for meaningful work in this area.

\section{CARBON DIOXIDE REMOVAL}

BY ANDREW S. ROSS \& EVAN D. SHERWIN

Even if we could cut emissions to zero today, we would still face significant climate consequences from GHGs already in the atmosphere. Eliminating emissions entirely may also be tricky, given the sheer diversity of sources (such as airplanes and cows). Instead, many experts argue that to meet critical climate goals, global emissions must become net-negative-that is, we must remove more $\mathrm{CO}_{2}$ from the atmosphere than we release $[259,269]$. Although there has been significant progress in negative emissions research $[260,540,575,580,707]$, the actual $\mathrm{CO}_{2}$ removal industry is still in its infancy. As such, many of the ML applications we outline in this section are either speculative or in the early stages of development or commercialization.

Many of the primary candidate technologies for $\mathrm{CO}_{2}$ removal directly harness the same natural processes which have (pre-)historically shaped our atmosphere. One of the most promising methods is simply allowing or encouraging more natural uptake of $\mathrm{CO}_{2}$ by plants (whose $\mathrm{ML}$ applications we discuss in Section 6). Other plant-based methods include bioenergy with carbon capture and biochar, where plants are grown specifically to absorb $\mathrm{CO}_{2}$ and then burned in a way that sequesters it (while creating energy or fertilizer as a useful byproduct) [155, 575, 690]. Finally, the way most of Earth's $\mathrm{CO}_{2}$ has been removed over geologic timescales is the slow process of mineral weathering, which also initiates further $\mathrm{CO}_{2}$ absorption in the ocean due to alkaline runoff [718]. These processes can both be massively accelerated by human activity to achieve necessary scales of $\mathrm{CO}_{2}$ removal [575]. However, although these biomass, mineral, and ocean-based methods are all promising enough as techniques to merit mention, they may have drawbacks in terms of land use and potentially serious environmental impacts, and (more relevantly for this article) they would not likely benefit significantly from ML.

\subsection{Direct Air Capture}

Long-term

Another approach is to build facilities to extract $\mathrm{CO}_{2}$ from power plant exhaust, industrial processes, or even ambient air [701]. While this direct air capture (DAC) approach faces technical hurdles, it requires little land and has, according to current understanding, minimal negative environmental impacts [152]. The basic idea behind DAC is to blow air onto $\mathrm{CO}_{2}$ sorbents (essentially like sponges, but for gas), which are either solid or in solution, then use heat-powered chemical processes to release the $\mathrm{CO}_{2}$ in purified form for sequestration [575, 707]. Several companies have recently been started to pilot these methods [112, 136, 293].

While $\mathrm{CO}_{2}$ sorbents are improving significantly [117, 872], issues still remain with efficiency and degradation over time, offering potential (though still speculative) opportunities for ML. ML could 
be used (as in Section 2.1.1) to accelerate materials discovery and process engineering workflows $[105,299,490,653]$ to maximize sorbent reusability and $\mathrm{CO}_{2}$ uptake while minimizing the energy required for $\mathrm{CO}_{2}$ release. ML might also help to develop corrosion-resistant components capable of withstanding high temperatures, as well as optimize their geometry for air-sorbent contact (which strongly impacts efficiency [350]).

\subsection{Sequestering $\mathrm{CO}_{2}$}

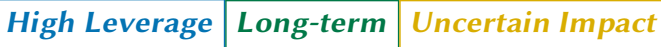

Once $\mathrm{CO}_{2}$ is captured, it must be sequestered or stored, securely and at scale, to prevent re-release back into the atmosphere. The best-understood form of $\mathrm{CO}_{2}$ sequestration is direct injection into geologic formations such as saline aquifers, which are generally similar to oil and gas reservoirs [575]. A Norwegian oil company has successfully sequestered $\mathrm{CO}_{2}$ from an offshore natural gas field in a saline aquifer for more than twenty years [895]. Another promising option is to sequester $\mathrm{CO}_{2}$ in volcanic basalt formations, which is being piloted in Iceland [744].

ML may be able to help with many aspects of $\mathrm{CO}_{2}$ sequestration. First, ML can help identify and characterize potential storage locations. Oil and gas companies have had promising results using ML for subsurface imaging based on raw seismograph traces [31]. These models and the data behind them could likely be repurposed to help trap $\mathrm{CO}_{2}$ rather than release it. Second, ML can help monitor and maintain active sequestration sites. Noisy sensor measurements must be translated into inferences about subsurface $\mathrm{CO}_{2}$ flow and remaining injection capacity [119]; recently, [542] found success using convolutional image-to-image regression techniques for uncertainty quantification in a global $\mathrm{CO}_{2}$ storage simulation study. Deep learning can also help speed up simulation of carbon dioxide plume migration in sequestration reservoirs [836]. Additionally, it is important to monitor for $\mathrm{CO}_{2}$ leaks [557]. ML techniques have recently been applied to monitoring potential $\mathrm{CO}_{2}$ leaks from wells [125]; computer vision approaches for emissions detection (see [826] and Section 6.1) may also be applicable.

\subsection{Discussion}

Given limits on how much more $\mathrm{CO}_{2}$ humanity can safely emit and the difficulties associated with eliminating emissions entirely, $\mathrm{CO}_{2}$ removal may have a critical role to play in tackling climate change. Promising applications for $\mathrm{ML}$ in $\mathrm{CO}_{2}$ removal include informing research and development of novel component materials, characterizing geologic resource availability, and monitoring underground $\mathrm{CO}_{2}$ in sequestration facilities. Although many of these applications are speculative, the industry is growing, which will create more data and more opportunities for ML approaches to help.

\section{CLIMATE PREDICTION}

\section{BY KELLY KOCHANSKI}

The first global warming prediction was made in 1896, when Arrhenius estimated that burning fossil fuels could eventually release enough $\mathrm{CO}_{2}$ to warm the Earth by $5^{\circ} \mathrm{C}$. The fundamental physics underlying those calculations has not changed, but our predictions have become far more detailed and precise. The predominant predictive tools are climate models, known as General Circulation Models or Earth System Models. ${ }^{25}$ These models inform local and national government decisions (see IPCC reports [370-372]), help people calculate their climate risks (see Sections 11 and 9) and allow us to estimate the potential impacts of solar geoengineering (see Section 10).

Recent trends have created opportunities for ML to advance the state-of-the-art in climate prediction (Figure 6). First, new and cheaper satellites are creating petabytes of climate observation

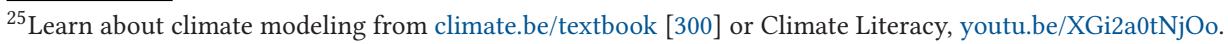




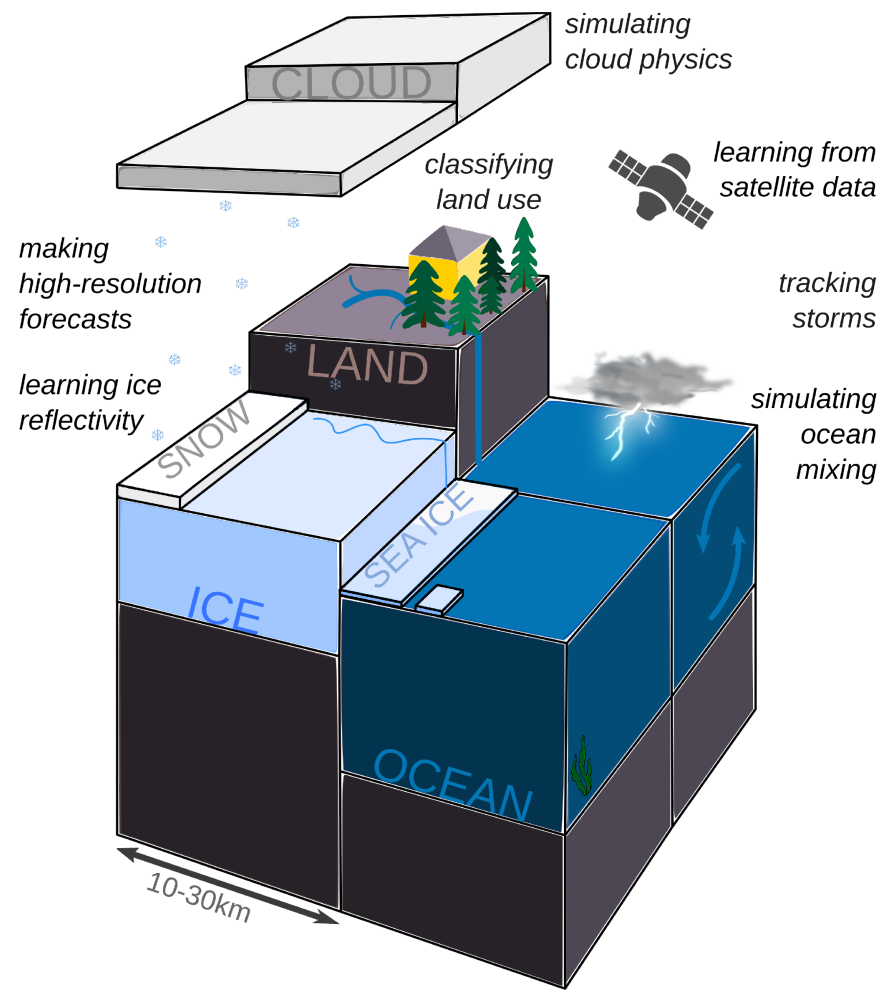

Fig. 6. Schematic of a climate model, with selected opportunities to improve climate change predictions using ML, as described in Section 8.

data. ${ }^{26}$ Second, massive climate modeling projects are generating petabytes of simulated climate data. ${ }^{27}$ Third, climate forecasts are computationally expensive [115] (the simulations in [415] took three weeks to run on NCAR supercomputers), while ML methods are becoming increasingly fast to train and run, especially on next-generation computing hardware. As a result, climate scientists have recently begun to explore ML techniques, and are starting to team up with computer scientists to build new and exciting applications.

\subsection{Uniting Data, ML, and Climate Science}

Climate models represent our understanding of Earth and climate physics. We can learn about the Earth by collecting data. To turn that data into useful predictions, we need to condense it into coherent, computationally tractable models. ML models are likely to be more accurate or less expensive than other models where: (1) there are plentiful data, but it is hard to model systems with traditional statistics, or (2) there are good models, but they are too computationally expensive to use in production.

8.1.1 Data for Climate Models. When data are plentiful, climate scientists build data-driven models. In these areas, ML techniques may solve many problems that were previously

\footnotetext{
${ }^{26}$ E.g., NASA's Earth Science Data Systems program, earthdata.nasa.gov, and ESA's Earth Online, earth.esa.int.

${ }^{27}$ E.g., the Coupled Model Intercomparison Project, cmip.llnl.gov [230, 781] and Community Earth System Model Large Ensemble [415].
} 
challenging. These include black box problems, for instance sensor calibration [468], and classification of observational data, for instance classifying land cover or identifying pollutant sources in satellite imagery [457, 469]. More applications like these are likely to appear as satellite databases grow. The authors of [548] describe many opportunities for data scientists to assimilate data from diverse field and remote sensing sources, many of which have since been explored by climate informatics researchers.

Numerous authors, such as [287], have identified geoscience problems that would be aided by the development of benchmark datasets. Efforts to develop such datasets include EnviroNet [565], the IS-GEO benchmark datasets [211], and ExtremeWeather [652]. We expect the collection of curated geoscience datasets to continue to grow; this process might even be accelerated by ML optimizations in data collection systems [287]. We strongly encourage modellers to dive into the data in collaboration with domain experts. We also recommend that modellers who seek to learn directly from data see [354] for specific advice on fitting and over-fitting climate data.

8.1.2 Accelerating Climate Models. Many climate prediction problems are irremediably datalimited. No matter how many weather stations we construct, how many field campaigns we run, or how many satellites we deploy, the Earth will generate at most one year of new climate data per year. Existing climate models deal with this limitation by relying heavily on physical laws, such as thermodynamics [214, 300]. These models are structured in terms of coupled partial differential equations that represent physical processes like cloud formation, ice sheet flow, and permafrost melt. ML models provide new techniques (e.g., [658]) for solving such systems efficiently.

\section{Clouds and aerosols.}

High Leverage

Recent work has shown how deep neural networks could be combined with existing thermodynamics knowledge to fix the largest source of uncertainty in current climate models: clouds. Bright clouds block sunlight and cool the Earth; dark clouds catch outgoing heat and keep the Earth warm [371, 729]. These effects are controlled by small-scale processes such as cloud convection and atmospheric aerosols (see uses of aerosols for cloud seeding and solar geoengineering in Section 10). Physical models of these processes are far too computationally expensive to include in global climate models-but ML models are not. Gentine et al. trained a deep neural network to emulate the behavior of a high-resolution cloud simulation, and found that the network gave similar results for a fraction of the cost [275] and was stable in a simplified global model [670]. Existing scientific model structures do not always offer great trade-offs between cost and accuracy. Neural networks trained on those scientific models produce similar predictions, but offer an entirely new set of compromises between training cost, production cost, and accuracy. Replacing select climate model components with neural network approximators may thus improve both the cost and the accuracy of global climate models. Additional work is needed to identify more climate model components that could be replaced by neural networks (we highlight other impactful components below), to optimize those models, and to automate their training workflows (see examples in [676]).

Ice sheets and sea level rise.

High Leverage

The next most important targets for climate model improvements are ice sheet dynamics and sea level rise. The Arctic and Antarctic are warming faster than anywhere else on Earth, and their climates control the future of global sea level rise and many vulnerable ecosystems [370, 372]. Unfortunately, these regions are dark and cold, and until recently they were difficult to observe. In the past few years, however, new satellite campaigns have illuminated them with hundreds 
of terabytes of data. ${ }^{28}$ These data could make it possible to use ML to solve some of the field's biggest outstanding questions. In particular, models of mass loss from the Antarctic ice-sheet are highly uncertain [449] and models of the extent of Antarctic sea ice do not match reality well [263]. The most uncertain parts of these models, and thus the best targets for improvement, are snow reflectivity, sea ice reflectivity, ocean heat mixing, and ice sheet grounding line migration rates $[328,354,449]$. Computer scientists who wish to work in this area could build models that learn snow and sea ice properties from satellite data, or use new video prediction techniques to predict short-term changes in the sea ice extent.

8.1.3 Working with Climate Models. ML could also be used to identify and leverage relationships between climate variables. Pattern recognition and feature extraction techniques could allow us to identify more useful connections in the climate system, and regression models could allow us to quantify non-linear relationships between connected variables. For example, Nowack et al. demonstrated that ozone concentrations could be computed as a function of temperature, rather than physical transport laws, which led to considerable computational savings [587].

The best climate predictions are synthesized from ensembles of $20+$ climate models [782]. Making good ensemble predictions is an excellent ML problem. Monteleoni et al. proposed that online ML algorithms could create better predictions of one or more target variables in a multi-model ensemble of climate models [549]; this idea has been refined in [530, 763]. More recently, Anderson and Lucas used random forests to make high-resolution predictions from a mix of high- and low-resolution models, which could reduce the costs of building multi-model ensembles [23].

In the further future, the Climate Modeling Alliance has proposed to build an entirely new climate model that learns continuously from data and from high-resolution simulations [717]. The proposed model would be written in Julia, in contrast to existing models which are mostly written in $\mathrm{C}++$ and Fortran. At the cost of a daunting translation workload, they aim to build a model that is more accessible to new developers and more compatible with ML libraries.

\subsection{Forecasting Extreme Events}

For most people, extreme event prediction means the local weather forecast and a few days' warning to stockpile food, go home, and lock the shutters. Weather forecasts are shorter-term than climate forecasts, but they produce abundant data. Weather models are optimized to track the rapid, chaotic changes of the atmosphere; since these changes are fast, tomorrow's weather forecast is made and tested every day. Climate models, in contrast, are chaotic on short time scales, but their long-term trends are driven by slow, predictable changes of ocean, land, and ice (see [734]). ${ }^{29}$ As a result, climate model output can only be tested against long-term observations (at the scale of years to decades). Intermediate time scales, of weeks to months, are exceptionally difficult to predict, although Cohen et al. [139] argue that ML could bridge that gap by making good predictions on four to six week timescales [363]. Thus far, however, weather modelers have had hundreds of times more test data than climate modelers, and began to adopt ML techniques earlier. Numerous ML weather models are already running in production. For example, Gagne et al. recently used an ensemble of random forests to improve hail predictions within a major weather model [262].

A full review of the applications of ML for extreme weather forecasting is beyond the scope of this article. Fortunately, that review has already been written, see [529]. The authors describe ML

\footnotetext{
${ }^{28}$ See, e.g., icebridge.gsfc.nasa.gov and pgc.umn.edu/data/arcticdem.

${ }^{29} \mathrm{This}$ is one of several reasons why climate models produce accurate long-term predictions in spite of atmospheric chaos.
} 
systems that correct bias, recognize patterns, and predict storms. Moving forward, they envision human experts working alongside automated forecasts.

8.2.1 Storm Tracking. Climate models cannot predict the specific dates of future events, but they can predict changes in long-term trends like drought frequency and storm intensity. Information about these trends helps individuals, corporations, and towns make informed decisions about infrastructure, asset valuation and disaster response plans (see also Section 9.4). Identifying extreme events in climate model output, however, is a classification problem with a twist: all of the available datasets are strongly skewed because extreme events are, by definition, rare. ML has been used successfully to classify some extreme weather events. Researchers have used deep learning to classify [488], detect [652], and segment [456] cyclones and atmospheric rivers, as well as tornadoes [463], in historical climate datasets. Tools for more event types would be useful, as would online tools that work within climate models, labelled datasets for predicting future events, and statistical tools that quantify the uncertainty in new extreme event forecasts.

\subsubsection{Local Forecasts.}

High Leverage

Forecasts are most actionable if they are specific and local. ML is widely used to make local forecasts from coarse 10-100 km climate or weather model predictions; various authors have attempted this using support vector machines, autoencoders, Bayesian deep learning, and super-resolution convolutional neural networks (e.g., [481]). Several groups are now working to translate highresolution climate forecasts into risk scenarios. For example, ML can predict localized flooding patterns from past data [625], which could inform individuals buying insurance or homes. Since ML methods like neural networks are effective at predicting local flooding during extreme weather events [740], these could be used to update local flood risk estimates to benefit individuals. The start-up Jupiter Intelligence is working to make climate predictions more actionable by translating climate forecasts into localised flood and temperature risk scores.

\subsection{Discussion}

ML may change the way that scientific modeling is done. The examples above have shown that many components of large climate models can be replaced with ML models at lower computational costs. From an ML standpoint, learning from an existing model has many advantages: modelers can generate new training and test data on-demand, and the new ML model inherits some community trust from the old one. This is an area of active ML research. Several papers have explored data-efficient techniques for learning dynamical systems [658], including physics-informed neural networks [659] and neural ordinary differential equations [132]; applications of physics-informed ML in climate science are now maturing rapidly [373, 412]. In the further future, researchers are developing ML approaches for a wide range of scientific modeling challenges, including crash prediction [497], adaptive numerical meshing [389], uncertainty quantification [464, 486], and performance optimization [786]. If these strategies are effective, they may solve some of the largest structural challenges facing current climate models.

New ML models for climate will be most successful if they are closely integrated into existing scientific models. This has been emphasized, again and again, by authors who have laid future paths for artificial intelligence within climate science [287, 470, 529, 670, 676, 717]. New models need to leverage existing knowledge to make good predictions with limited data. In 10 years, we will have more satellite data, more interpretable ML techniques [791], hopefully more trust from the scientific community, and possibly a new climate model written in Julia. For now, however, ML models must be creatively designed to work within existing climate models. The best of these models are likely to be built by close-knit teams including both climate and computational scientists. 


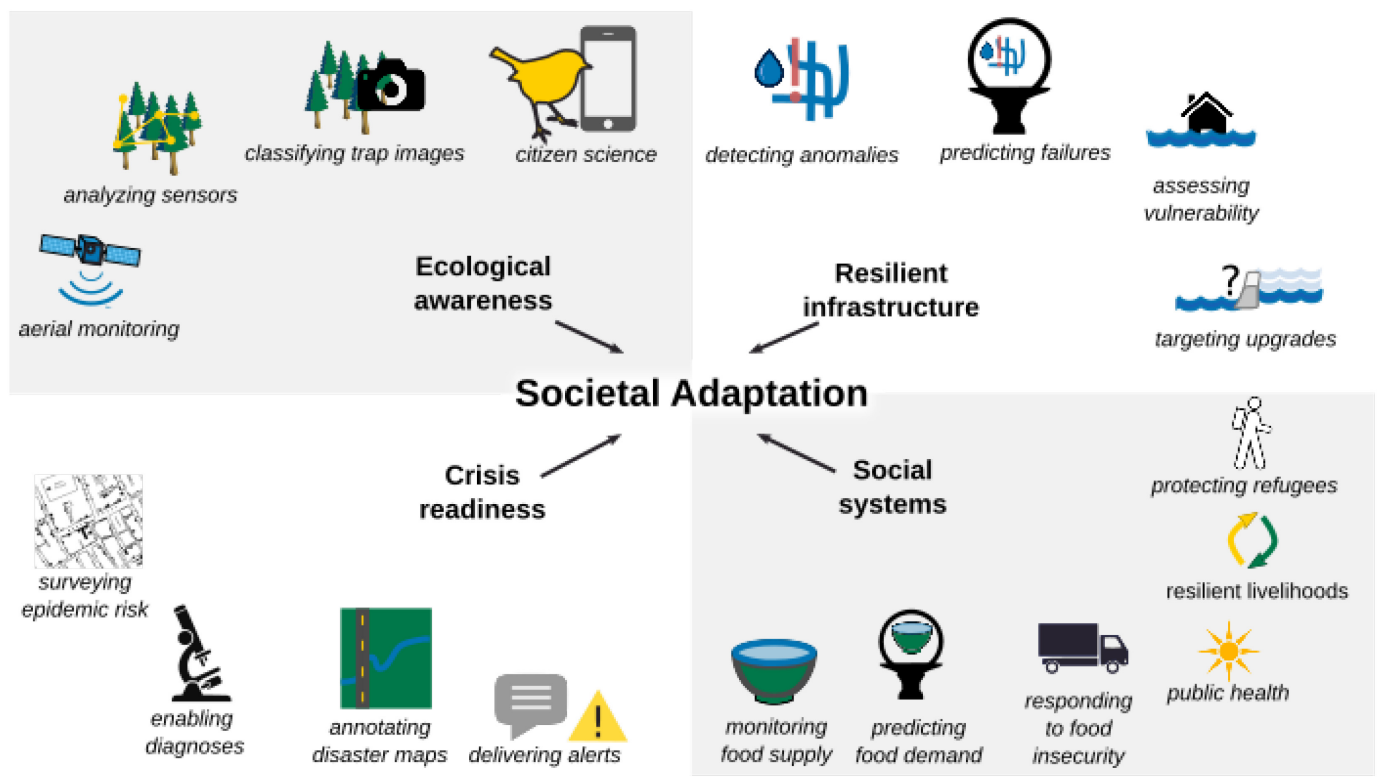

Fig. 7. Selected opportunities to accelerate societal adaptation to climate change using ML, as described in Section 9.

\section{SOCIETAL IMPACTS}

BY KRIS SANKARAN

Changes in the atmosphere have impacts on the ground. The expected societal impacts of climate change include prolonged ecological and socioeconomic stresses as well as brief, but severe, societal disruptions. For example, impacts could include both gradual decreases in crop yield and localized food shortages. If we can anticipate climate impacts well enough, then we can prepare for them by asking:

- How do we reduce vulnerability to climate impacts?

- How do we support rapid recovery from climate-induced disruptions?

A wide variety of strategies have been put forward, from robust power grids to food shortage prediction (Figure 7), and while this is good news for society, it can be overwhelming for an ML practitioner hoping to contribute. Fortunately, a few critical needs tend to recur across strategiesit is by meeting these needs that ML has the greatest potential to support societal adaptation [251, 296, 648]. From a high level, these involve

- Sounding alarms: Identifying and prioritizing the areas of highest risk, by using evidence of risk from historical data.

- Providing annotation: Extracting actionable information or labels from unstructured raw data.

- Promoting exchange: Making it easier to share resources and information to pool and reduce risk.

These unifying threads will appear repeatedly in the sections below, where we review strategies to help ecosystems, infrastructure, and societies adapt to climate change, and explain how ML supports each strategy (Figure 7). 
We note that the projects involved vary in scale from local to global, from infrastructure upgrades and crisis preparedness planning to international ecosystem monitoring and disease surveillance. Hence, we anticipate valuable contributions by researchers who have the flexibility to formulate experimental approaches, by industrial engineers and entrepreneurs who have the expertise to translate prototypes into wide-reaching systems, and by civil servants who lead many existing climate adaptation efforts.

\subsection{Ecology}

Changes in climate are increasingly affecting the distribution and composition of ecosystems. This has profound implications for global biodiversity, as well as agriculture, disease, and natural resources such as wood and fish. ML can help by supporting efforts to monitor ecosystems and biodiversity.

Monitoring ecosystems.

High Leverage

To preserve ecosystems, it is important to know which are most at risk. This has traditionally been done via manual, on-the-ground observation, but the process can be accelerated by annotation of remote sensing data [91, 92, 513, 637] (see also Section 6.1). For example, tree cover can be automatically extracted from aerial imagery to characterize deforestation $[362,528]$. At the scale of regions or biomes, analysis of large-scale simulations can illuminate the evolution of ecosystems across potential climate futures [238, 438]. A more direct source of data is offered by environmental sensor networks, made from densely packed but low-cost devices [184, 331, 361]. To monitor ocean ecosystems, marine robots are useful, because they can be used to survey large areas on demand $[208,306]$.

For a system to have the most real-world impact, regardless of the underlying data source, it is necessary to "personalize" predictions across a range of ecosystems. A model trained on the Sahara would almost certainly fail if deployed in the Amazon. Hence, these applications may motivate ML researchers interested in heterogeneity, data collection, transfer learning, and rapid generalization. In sensor networks, individual nodes fail frequently, but are redundant by design-this is an opportunity for research into anomaly detection and missing data imputation [178, 345]. In marine robotics, improved techniques for sampling regions to explore and automatic summarization of expedition results would both provide value $[160,246]$. Finally, beyond aiding adaptation by prioritizing at-risk environments, the design of effective methods for ecosystem monitoring will support the basic science necessary to shape adaptation in the long-run [231, 297, 521].

Monitoring biodiversity.

High Leverage Accurate estimates of species populations are the foundation on which conservation efforts are built. Camera traps and aerial imagery have increased the richness and coverage of sampling efforts. ML can help infer biodiversity counts from image-based sensors. For instance, camera traps take photos automatically whenever a motion sensor is activated-computer vision can be used to classify the species that pass by, supporting a real-time, less labor-intensive species count $[64,585,643]$. It is also possible to use aerial imagery to estimate the size of large herds [805] or count birds [282]. In underwater ecosystems, ML has been used to identify plankton automatically from underwater cameras [232] and to infer fish populations from the structure of coral reefs [867].

Citizen science can also enable dataset collection at a scale impossible in individual studies [93, 533, 634, 767]. For example, by leveraging public enthusiasm for birdwatching, eBird has logged more than 140 million observations [767], which have been used for population and migration studies [424]. Computer vision algorithms that can classify species from photographs have furthered such citizen science efforts by making identifications easier and more accurate [660, 806], 
though these face challenges such as class imbalances in training data [807]. Work with citizen science data poses the additional challenge that researchers have no control over where samples come from. To incentivize observations from undersampled regions, mechanisms from game theory can be applied [860], and even when sampling biases persist, estimates of dataset shift can minimize their influence [128].

Monitoring biodiversity may be paired with interventions to protect rare species or control invasive pests. ML is providing new solutions to assess the impact of ecological interventions $[13,502,664]$ and prevent poaching [860].

\subsection{Infrastructure}

Physical infrastructure is so tightly woven into the fabric of everyday life-like the buildings we inhabit and lights we switch on-that it is easy to forget that it exists (see Section 4). The fact that something so basic will have to be rethought in order to adapt to climate change can be unsettling, but viewed differently, the sheer necessity of radical redesign can inspire creative thinking.

We first consider the impacts of climate change on the built environment. Shifts in weather patterns are likely to put infrastructure under more persistent stress. Heat and wind damage roads, buildings, and power lines. Rising water tables near the coast will lead to faults in pipelines. Urban heat islands will be exacerbated and it is likely that there will be an increased risk of flooding caused by heavy rain or coastal inundations, resulting in property damage and traffic blockages [604].

A clear target is construction of physical defenses, for example, "climate proofing" cities with new coastal embankments and increased storm drainage capacity. However, focusing solely on defending existing structures can stifle proactive thinking about urban and social developmentfor example, floating buildings are being tested in Rotterdam-and one may alternatively consider resilience and recovery more broadly [621,731]. From this more general perspective of improving social processes, ML can support two types of activities: design and maintenance.

\section{Designing infrastructure.}

Long-term

How can infrastructure be (re)designed to dampen climate impacts? In road networks, it is possible to incorporate flood hazard and traffic information in order to uncover vulnerable stretches of road, especially those with few alternative routes [314]. If traffic data are not directly available, it is possible to construct proxies from mobile phone usage and city-wide CCTV streams-these are promising in rapidly developing urban centers [252, 381]. Overall flood hazard maps can be improved using ML [455], and it is also possible to leverage data from real-world flooding events [616], and to send localized predictions to those at risk [841]. For electrical, water, and waste collection networks, the same principle can guide investments in resilience-using proxy or historical data about disruptions to anticipate vulnerabilities [564, 574, 600, 609]. Robust components can replace those at risk; for example, adaptive islands, parts of an energy grid that continue to provide power even when disconnected from the network, prevent cascading outages in power distribution [235].

Infrastructure is long-lived, but the future is uncertain, and planners must weigh immediate resource costs against future societal risks [248]. One area that urgently needs adaptation strategies is the consistent access to drinking water, which can be jeopardized by climate variability [174, 366]. Investments in water infrastructure can be optimized; for example, a larger dam might cost more up front, but would have a larger storage capacity, giving a stronger buffer against drought. To delay immediate decisions, infrastructure can be upgraded in phases-the technical challenge is to discover policies that minimize a combination of long-term resource and societal costs under plausible climate futures, with forecasts being updated as climates evolve [289, 651, 727]. 
Maintaining infrastructure.

High Leverage

What types of systems can keep infrastructure functioning well under increased stress? Two strategies for efficiently managing limited maintenance resources are predictive maintenance and anomaly detection; both can be applied to electrical, water, and transportation infrastructure (see also Sections 2.2 and 5.3). In predictive maintenance, operations are prioritized according to the predicted probability of a near-term breakdown [201, 581, 702, 751]. For anomaly detection, failures are discovered as soon as they occur, without having to wait for inspectors to show up, or complaints to stream in $[51,186]$.

The systems referenced here have required the manual curation of data streams, structured and unstructured. The data are plentiful, just difficult to glue together. Ideas from the missing data, multimodal data, and AutoML communities have the potential to resolve some of these issues.

\subsection{Social Systems}

While less tangible, the social systems we construct are just as critical to the smooth functioning of society as any physical infrastructure, and it is important that they adapt to changing climate conditions. First, consider what changes these systems may encounter. Decreases in crop yield, due to drought and other factors, will pose a threat to food security, as already evidenced by long periods of drought in North America, West Africa, and East Asia [157, 636]. More generally, communities dependent on ecosystem resources will find their livelihoods at risk, and this may result in mass migrations, as people seek out more supportive environments.

At first, these problems may seem beyond the reach of algorithmic thinking, but investments in social infrastructure can increase resilience. ML can amplify the reach and effectiveness of this infrastructure. See also Section 12 for perspective on how ML can support the function and analysis of complex social environments.

Food security.

High Leverage

Data can be used to monitor the risk of food insecurity in real time, to forecast near-term shortages, and to identify areas at risk in the long-term, all of which can guide interventions. For real-time and near-term systems, it is possible to distill relevant signals from mobile phones, credit card transactions, and social media data [171, 434, 645]. These have emerged as low-cost, high-reach alternatives to manual surveying. The idea is to train models that link these large, but decontextualized, data with ground truth consumption or survey information, collected on small representative samples. This process of developing proxies to link small, rich datasets with large, coarse ones can be viewed as a type of semi-supervised learning, and is fertile ground for research.

For longer-term warnings, spatially localized crop yield predictions are needed. These can be generated by aerial imagery or meteorological data (see Section 6.2), if they can be linked with historical yield data $[123,824]$. Automatic crop type mapping can also be a valuable tool for yield prediction [426, 427]. On the ground, it is possible to perform crop-disease identification from plant photos-this can alert communities to disease outbreaks, and enhance the capacity of agricultural inspectors. For even longer-run risk evaluation, it is possible to simulate crop yield via biological and ecological models [446, 698, 783], presenting another opportunity for blending large scale simulation with ML $[605,835]$.

Beyond sounding alarms, ML can improve resilience of food supply chains. As detailed in Section 5, ML can reduce waste along these chains; we emphasize that for adaptation, it is important that supply chains also be made robust to unexpected disruptions [203, 568, 639, 665].

Resilient livelihoods.

Individuals whose livelihoods depend on one activity, and who have less access to community 
resources, are those who are most at risk [7, 692]. Resilient livelihoods can be promoted through increased diversification, cooperation, and exchange, all of which can be facilitated by ML systems. For example, they can guide equipment and information sharing in farming cooperatives, via growers' social networks [37]. Mobile money efforts can increase access to liquid purchasing power; they can also be used to monitor economic health [253, 644]. Skill-matching programs and online training are often driven by data, with some programs specifically aiming to benefit refugees $[54,516,646]$ (see also Section 13 ).

\section{Supporting displaced people.}

Long-term Uncertain Impact Human populations move in response to threats and opportunities, and ML can be used to predict large-scale migration patterns. Work in this area has relied on accessible proxies, like social media, where users' often self-report location information, or aerial imagery, from which the extent of informal settlement can be gauged [82, 376, 650, 869]. More than quantifying migration patterns, there have been efforts directly aimed at protecting refugees, either through improving rescue operations [492, 628] or monitoring negative public sentiment [647]. It is worth cautioning that immigrants and refugees are vulnerable groups, and systems that surveil them can easily be exploited by bad actors. Designing methodology and governance mechanisms that allow vulnerable populations to benefit from such data, without putting them at additional risk, should be a research priority.

Assessing health risks.

Climate change will affect exposure to health hazards, and ML can play a role in measuring and mitigating their impacts across subpopulations. Two of the most relevant expected shifts are (1) heat waves will become more frequent and (2) outdoor and indoor air quality will deteriorate $[324,710]$. These exposures have either direct or indirect effects on health. For example, prolonged heat episodes both directly cause heat stroke and can trigger acute episodes in chronic conditions, like heart or respiratory disease [194, 719].

Careful data collection and analysis have played a leading role in epidemiology and public health efforts for generations. It should be no surprise that ML has emerged as an important tool in these disciplines, supporting a variety of research efforts, from increasing the efficiency of disease simulators to supporting the fine-grained measurement of exposures and their health impacts [433, 705].

These disciplines are increasingly focused on the risks posed by climate change specifically. For example, new sources of data have enabled detailed sensing of urban heat islands [137, 348, 815], water quality [321, 440], and air pollution [130, 180]. Further, data on health indicators, which are already collected, can quantitatively characterize observed impacts across regions as well as illuminate which populations are most at risk to climate-change induced health hazards [831]. For example, it is known that the young, elderly, and socially isolated are especially vulnerable during heat waves, and finer-grained risk estimates could potentially drive outreach [622, 697].

Across social applications, there are worthwhile research challenges-guiding interventions based on purely observational, potentially unrepresentative data poses risks. In these contexts, transparency is necessary, and ideally, causal effects of interventions could be estimated, to prevent feedback loops in which certain subgroups are systematically ignored from policy interventions.

\subsection{Crisis}

Perhaps counterintuitively, natural disasters and health crises are not entirely unpredictable-they can be prepared for, risks can be reduced, and coordination can be streamlined. Furthermore, while crises may be some of the most distressing consequences of climate change, disaster response and 
public health are mature disciplines in their own right, and have already benefited extensively from ML methodology $[118,531,862]$.

\section{Managing epidemics.}

Climate change will increase the range of vector and water-borne diseases, elevating the likelihood that these new environments experience epidemics [324]. Disease surveillance and outbreak forecasting systems can be built from web data and specially-designed apps, in addition to traditional surveys [394, 467, 627]. While non-survey proxies are observational and self-reported, current research attempts to address these issues $[472,588]$. Beyond surveillance, point-of-care diagnostics have enjoyed a renaissance, thanks in part to ML $[598,648]$. These are tools that allow health workers to make diagnoses when specialized lab equipment is inaccessible. An example is malaria diagnosis based on photos of prepared pathology slides taken with a mobile phone [649]. Ensuring that these systems reliably and transparently augment extension workers, guiding data collection and route planning when appropriate, are active areas of study [97, 688].

Disaster response.

High Leverage

In disaster preparation and response, two types of ML tasks have proven useful: creating maps from aerial imagery and performing information retrieval on social media data. Accurate and wellannotated maps can inform evacuation planning, retrofitting campaigns, and delivery of relief $[59,200]$. Further, this imagery can assist damage assessment, by comparing scenes immediately pre- and post-disaster $[315,816]$. Social media data can contain kernels of insight-places without water, clinics without supplies-which can inform relief efforts. ML can help properly surface these insights, compressing large volumes of social media data into the key takeaways, which can be acted upon by disaster managers [118, 368, 596].

\subsection{Discussion}

Climate change will have profound effects on the planet, and the ML community can support efforts to minimize the damage it does to ecosystems and the harm it inflicts on people. This section has suggested areas of research that may help societies adapt more effectively to these ever changing realities. We have identified a few recurring themes, but also emphasized the role of understanding domain-specific needs. The use of ML to support societal resilience would be a noble goal at any time, but the need for tangible progress towards it may never have been so urgent as it is today, in the face of the wide-reaching consequences of climate change.

\section{SOLAR GEOENGINEERING}

BY ANDREW S. ROSS

Airships floating through the sky, spraying aerosols; robotic boats crisscrossing the ocean, firing vertical jets of spray; arrays of mirrors carefully positioned in space, micro-adjusted by remote control; these images seem like science fiction, but they are actually real proposals for solar radiation management, commonly called solar geoengineering [375, 418, 419, 728]. Solar geoengineering, much like the GHGs causing climate change, shifts the balance between how much heat the Earth absorbs and how much it releases. The difference is that it is done deliberately, and in the opposite direction. The most common umbrella strategy is to make the Earth more reflective, keeping heat out, though there are also methods of helping heat escape (besides $\mathrm{CO}_{2}$ removal, which we discuss in Sections 6 and 7).

Solar geoengineering generally comes with a host of potential side effects and governance challenges. Moreover, unlike $\mathrm{CO}_{2}$ removal, it cannot simply reverse the effects of climate change (average temperatures may return to pre-industrial levels, but location-specific climates still change), and also comes with the risk of termination shock (fast, catastrophic warming if humanity 
undertakes solar geoengineering but stops suddenly) [615]. Because of these and other issues, it is not within the scope of this article to evaluate or recommend any particular technique. However, the potential for solar geoengineering to moderate some of the most catastrophic hazards of climate change is well-established [374], and it has received increasing attention in the wake of societal inaction on mitigation. Although [418] argue that the "hardest and most important problems raised by solar geoengineering are non-technical," there are still a number of important technical questions that ML may be able to help us study.

\section{Overview.}

The primary candidate methods for geoengineering are marine cloud brightening [396] (making low-lying clouds more reflective), cirrus thinning [759] (making high-flying clouds trap less heat), and stratospheric aerosol injection [668] (which we discuss below). Other candidates (which are either less effective or harder to implement) include "white-roof" methods [10] and even launching sunshades into space [29].

Injecting sulfate aerosols into the stratosphere is considered a leading candidate for solar geoengineering both because of its economic and technological feasibility [527, 743] and because of a reason that should resonate with the ML community: we have data. (These data are largely in the form of temperature observations after volcanic eruptions, which release sulfates into the stratosphere when sufficiently large [691].) Once injected, sulfates circulate globally and remain aloft for 1 to 2 years. As a result, the process is reversible, but must also be continually maintained. Sulfates come with a well-studied risk of ozone loss [210], and they make sunlight slightly more diffuse, which can impact agriculture [642].

\subsection{Understanding and Improving Aerosols}

Design.

Long-term

The effects and side-effects of aerosols in the stratosphere (or at slightly lower altitudes for cirrus thinning [308]) vary significantly with their optical and chemical properties. Although sulfates are the best understood due to volcanic eruption data, many others have been studied, including zirconium dioxide, titanium dioxide, calcite (which preserves ozone), and even synthetic diamond [209]. However, the design space is far from fully explored. ML has had recent success in predicting specific chemical, material, and optical properties without the need for expensive experimentation or brute-force simulation [105, 299, 490, 653], including in aerosols [465, 500]. Although speculative, it is conceivable that ML could accelerate the search for aerosols that are chemically nonreactive but still reflective, cheap, and easy to keep aloft.

\section{Modeling.}

One reason that sulfates have been the focus for aerosol research is that atmospheric aerosol physics is not perfectly captured by current climate models, so having natural data is important for validation. Furthermore, even if current aerosol models are correct, their best-fit parameters must still be determined (using historical data), which comes with uncertainty and computational difficulty. ML may offer tools here, both to help quantify and constrain uncertainty, and to manage computational load. As a recent example, [247] use Gaussian processes to emulate climate model outputs based on nine possible aerosol parameter settings, allowing them to establish plausible parameter ranges (and thus much better calibrated error-bars) with only 350 climate model runs instead of $>100,000$. Although this is important progress, ideally we want uncertainty-aware aerosol simulations with a fraction of the cost of one climate model run, rather than 350. ML may be able to help here too (see Section 8 for more details). 


\subsection{Engineering a Control System}

\begin{tabular}{|l|l|l|}
\hline High Leverage & Long-term & Uncertain Impact
\end{tabular}

Efficient emulations and error-bars will be essential for what MacMartin and Kravitz [508] call "The Engineering of Climate Engineering." According to [508], any practical deployment of geoengineering would constitute "one of the most critical engineering design and control challenges ever considered: making real-time decisions for a highly uncertain and nonlinear dynamic system with many input variables, many measurements, and a vast number of internal degrees of freedom, the dynamics of which span a wide range of timescales." Bayesian and neural network-based approaches could facilitate the fast, uncertainty-aware nonlinear system identification this challenge might require. Additionally, there has been recent progress in RL for control [21, 84, 545], which could be useful for fine-tuning geoengineering interventions such as deciding where and when to release aerosols. For an initial attempt at analyzing stratospheric aerosol injection as a RL problem (using a neural network climate model emulator), see [170].

\subsection{Modeling Impacts}

Long-term

Of course, optimizing interventions requires defining objectives, and the choices here are far from clear. Although it is possible to stabilize global mean temperature and even regional temperatures through geoengineering, it is most likely impossible to preserve all relevant climate characteristics in all locations. Furthermore, climate model outputs do not tell the full story; ultimately, the goal of climate engineering is to minimize harm to people, ecosystems, and society. It is therefore essential to develop robust tools for estimating the extent and distribution of these potential harms. There has been some recent work in applying ML to assess the impacts of geoengineering. For example, [179] use deep neural networks to estimate the effects of aerosols on human health, while [148] use them to estimate the effects of solar geoengineering on agriculture. References $[101,187]$ use relatively simple local and polynomial regression techniques but applied to extensive empirical data to estimate the past and future effects of temperature change on economic production. More generally, the field of Integrated Assessment Modeling [422, 840] aims to map the outputs of a climate model to societal impacts; for a general discussion of potential opportunities for applying ML to integrated assessment models (IAMs), see Section 12.2.

\subsection{Discussion}

Any consideration of solar geoengineering raises many moral questions. It may help certain regions at the expense of others, introduce risks like termination shock, and serve as a "moral hazard": widespread awareness of its very possibility may undermine mainstream efforts to cut emissions [485]. Because of these issues, there has been significant debate about whether it is ethically responsible to research this topic [420, 640]. However, although it creates new risks, solar geoengineering could actually be a moderating force against the terrifying uncertainties climate change already introduces [374, 509], and ultimately many environmental groups and governmental bodies have come down on the side of supporting further research [145, 258, 592]. In this section, we have attempted to outline some of the technical challenges in implementing and evaluating solar geoengineering. We hope the ML community can help geoengineering researchers tackle these challenges.

\section{INDIVIDUAL ACTION}

BY NATASHA JAQUES

Individuals may worry that they are powerless to affect climate change, or lack clarity on which of their behaviors are most important to change. In fact, there are actions which can meaningfully reduce each person's carbon footprint, and, if widely adopted, could have a significant impact on 
mitigating global emissions [335, 845]. AI can help to identify those behaviors, inform individuals, and provide constructive opportunities by modeling individual behavior.

\subsection{Understanding Personal Carbon Footprint}

We as individuals are constantly confronted with decisions that affect our carbon footprint, but we may lack the data and knowledge to know which decisions are most impactful. Fortunately, ML can help determine an individual's carbon footprint from their personal and household data [790]. For example, natural language processing can be used to extract the flights a person takes from their email, or determine specific grocery items purchased from a bill, making it possible to predict the associated emissions. Systems that combine this information with data obtained from the user's smartphone (e.g., from a ride-sharing app) can then help consumers who wish to identify which behaviors result in the highest emissions. Given such a ML model, counterfactual reasoning can potentially be used to demonstrate to consumers how much their emissions would be reduced for each behavior they changed. As a privacy-conscious alternative, emissions estimates could be directly incorporated into grocery labels [562] or interfaces for purchasing flights. Such information can empower people to understand how they can best help mitigate climate change through behavior change.

Residences are responsible for a large share of GHG emissions [372] (see also Section 4). A large meta-analysis found that significant residential energy savings can be achieved [215], by targeting the right interventions to the right households [14, 16, 17]. ML can predict a household's emissions in transportation, energy, water, waste, foods, goods, and services, as a function of its characteristics [399]. These predictions can be used to tailor customized interventions for highemissions households [398]. Changing behavior both helps mitigate climate change and benefits individuals; studies have shown that many carbon mitigation strategies also provide cost savings to consumers [399].

Household energy disaggregation breaks down overall electricity consumption into energy use by individual appliances (see also Section 4.1) [33], which can help facilitate behavior change [772]. For example, it can be used to inform consumers of high-energy appliances of which they were previously unaware. This alone could have a significant impact, since many devices consume a large amount of electricity even when not in use; standby power consumption accounts for roughly $8 \%$ of residential electricity demand [507]. A variety of ML techniques have been used to effectively disaggregate household energy, such as spectral clustering, Hidden Markov Models, and neural networks [33].

ML can also be used to predict the marginal emissions of energy consumption in real time, on a scale of hours [832], potentially allowing consumers to effectively schedule activities such as charging an EV when the emissions (and prices [439]) will be lowest [143]. Combining these predictions with disaggregated energy data allows for the efficient automation of household energy consumption, ideally through products that present interpretable insights to the consumer (e.g., [721, 760]). Methods like RL can be used to learn how to optimally schedule household appliances to consume energy more efficiently and sustainably [543, 679]. Multi-agent learning has also been applied to this problem, to ensure that groups of homes can coordinate to balance energy consumption to keep peak demand low $[662,863]$.

\subsection{Facilitating Behavior Change}

High Leverage

ML is highly effective at modeling human preferences, and this can be leveraged to help mitigate climate change. Using ML, we can model and cluster individuals based on their climate knowledge, preferences, demographics, and consumption characteristics (e.g., [66, 116, 166, 261, 861]), and thus predict who will be most amenable to new technologies and sustainable behavior change. Such 
techniques have improved the enrollment rate of customers in an energy savings program by 2-3x [14]. Other works have used ML to predict how much consumers are willing to pay to avoid potential environmental harms of energy consumption [167], finding that some groups were totally insensitive to cost and would pay the maximum amount to mitigate harm, while other groups were willing to pay nothing. Given such disparate types of consumers, targeting interventions toward particular households may be especially worthwhile; all the more so because data show that the size and composition of household carbon footprints varies dramatically across geographic regions and demographics [399].

Citizens who would like to engage with policy decisions, or explore different options to reduce their personal carbon footprint, can have difficulty understanding existing laws and policies due to their complexity. They may benefit from tools that make policy information more manageable and relevant to the individual (e.g., based on where the individual lives). There is the potential for natural language processing to derive understandable insights from policy texts for these applications, similar to automated compliance checking $[67,876]$.

Understanding individual behavior can help signal how it can be nudged. For example, path analysis has shown that an individual's psychological distance to climate change (on geographic, temporal, social, and uncertainty dimensions) fully mediates their level of climate change concern [397]. This suggests that interventions minimizing psychological distance to the effects of climate change may be most effective. Similarly, ML has revealed that cross-cultural support for international climate programs is not reduced, even when individuals are exposed to information about other countries' climate behavior [65]. To make the effects of climate change more real for consumers, and thus help motivate those who wish to act, image generation techniques such as CycleGANs have been used to visualize the potential consequences of extreme weather events on houses and cities [716]. Gamification via deep learning has been proposed to further allow individuals to explore their personal energy usage [447]. All of these programs may be an incredibly cost-effective way to reduce energy consumption; behavior change programs can cost as little as 3 cents to save a kilowatt hour of electricity, whereas generating one kWh would cost 5-6 cents with a coal or wind power plant, and 10 cents with solar [207, 313].

\subsection{Discussion}

While individuals can sometimes feel that their contributions to climate change are dwarfed by other factors, in reality individual actions can have a significant impact in mitigating climate change. ML can aid this process by empowering consumers to understand which of their behaviors lead to the highest emissions, automatically scheduling energy consumption, and providing insights into how to facilitate behavior change.

\section{COLLECTIVE DECISIONS}

BY TEGAN MAHARAJ \& NIKOLA MILOJEVIC-DUPONT

Addressing climate change requires swift and effective decision-making by groups at multiple levels-communities, unions, NGOs, businesses, governments, intergovernmental organizations, and many more. Such collective decision-making encompasses many kinds of action-for example, negotiating international treaties to reduce GHG emissions, designing carbon markets, building resilient infrastructure, and establishing community-owned solar farms. These decisions often involve multiple stakeholders with different goals and priorities, requiring difficult trade-offs. The economic and societal systems involved are often extremely complex, and the impacts of climaterelated decisions can play out globally across long time horizons. To address some of these challenges, researchers are using empirical and mathematical methods from fields such as policy anal- 
ysis, operations research, economics, game theory, and computational social science; there are many opportunities for ML to support and supplement these methods.

\subsection{Modeling Social Interactions}

When designing climate change strategies, it is critical to understand how organizations and individuals act and interact in response to different incentives and constraints. Agent-based models (ABMs) $[168,222]$ represent one approach used in simulating the actions and interactions of agents (people, companies, etc.) in their environment. ABMs have been applied to a multitude of problems relevant to climate change, in particular to study low-carbon technology adoption [320, 584, 656, 878]. For example, when modeling solar PV adoption [875], agents may represent individuals who act based on factors such as financial interest and the behavior of their peers $[88,655]$; the goal is then to study how these agents interact in response to different conditions, such as electricity rates, subsidy programs, and geographical considerations. Here, ML can help identify the roles of these conditions directly from data [58]. Other applications of ABMs include modeling how behavior under social norms changes with external pressures [715], how the economy and climate may evolve given a diversity of political and economic beliefs [272], and how individuals may migrate in response to environmental changes [787]. While agent and environment models in ABMs are often hand-designed by experts, ML can help integrate data-driven insights into these models [874], for example, by learning rules or models for agents based on observational data $[312,875]$, or by using unsupervised methods such as variational autoencoders or generative adversarial networks to discover salient features useful in modeling a complex environment. While the hope of learning or tuning behavior from data is promising for generalization, many data-driven approaches lose the interpretability for which ABMs are valued; work in interpretable ML methods could potentially help with this.

In addition to ABMs, techniques from game theory can be valuable in modeling behavior, e.g., to explore cooperation in the face of a depleting resource [344]. Multi-agent RL can also be applied to understand the behavior of groups of agents who need to cooperate; see [607] for an overview and $[385,475]$ for recent examples. Combined with mechanism design,${ }^{30}$ such approaches can be used to design methods for cooperation that lead to mutually beneficial outcomes, for example when formalizing procedures around international climate agreements [522, 641].

\subsection{Informing Policy}

The actions required to address climate change, both in mitigation and adaptation, require making policies $^{31}$ at the local, national, and international levels [756]. Various institutions act as policy makers: for instance, governments, international organizations, non-governmental organizations, standards committees, and professional institutions. Tools from policy analysis-the process of evaluating the outcomes of past policies and assessing future policy alternatives ${ }^{32}-$ can help inform the choices these institutions make. Policy analysis uses quantitative tools from statistics, economics, and operations research such as cost-benefit analysis, uncertainty analysis, and multicriteria decision-making to inform the policymaking process; see [555, 618] for an introduction. ML can provide data for policy analysis, help improve existing tools for assessing policy options, and provide new tools for evaluating the effects of policies.

\footnotetext{
${ }^{30}$ Mechanism design is often called "inverse game theory"-rather than determining optimal strategies for players, mechanism design seeks to design games such that certain strategies are incentivized.

${ }^{31}$ Policy can refer, for example, to laws, measures, standards, or best practices.

${ }^{32}$ The former is often referred to as ex-post policy analysis and the latter as ex-ante policy analysis.
} 
Gathering data.

High Leverage

When creating policies, decision-makers must often negotiate fundamental uncertainties in the underlying data. ML can help alleviate some of this uncertainty by providing data. For instance, as detailed elsewhere in this article, ML can help pinpoint sources of emissions (Sections 2.2 and 6.1) approximate traffic patterns (Section 3.1), identify infrastructure at risk (Section 9.2), and mine information from companies' financial disclosures (Section 14). Natural language processing, network analysis, and clustering techniques can also be used to analyze social media data to understand public opinions and discourse around climate change [436, 812, 844]. These data can then be used to identify areas of intervention, compute the benefits and costs of a project, or evaluate the effectiveness of a policy after it has been implemented.

Assessing policy options.

Decision-makers often construct mathematical models to help them assess or tradeoff between different policy alternatives. ML is particularly relevant to approaches that model large and complex socio-economic systems to assess outcomes of particular strategies, as well as optimization-based tools that help with navigating the decision.

Policy-makers often wish to analyze how different policy alternatives may contribute to achieving a particular objective. Computational approaches such as simulation and (partial) equilibrium models can be used to compare different policy options, assess the effects of underlying assumptions, or propose strategies that are consistent with the objectives of decision-makers. Of particular relevance to climate change mitigation are IAMs, which incorporate economic models, climate models, and policy information (see [840] for an overview). IAMs are used to explore future societal pathways that are consistent with climate goals (e.g., $1.5^{\circ} \mathrm{C}$ mean global temperature increase), and play a prominent role in the IPCC assessments [561]. While these models can simulate interactions between many variables in great detail, this comes at the cost of computational complexity and presents opportunities for ML. Much as with Earth system models (Section 8), ML can be applied within any of the various sub-models that make up an IAM. One set of applications involves deriving results at the appropriate spatial resolution, since different components of an IAM operate at different scales. Outputs with high resolution may be aggregated via clustering methods to provide insights [183], while at coarser resolution, statistical downscaling can help to disaggregate data to an appropriate spatial resolution, as seen in applications such as crop yield [249], wind speed [479] or surface temperature [481]. ML also has the potential to help with sensitivity and uncertainty analysis [386], with finding numerical solutions for computational expensive submodels [206, 714], and assessing the validity of the models [556].

In addition to assessing the outcomes of various policies, policymakers may also employ optimization-based tools to figure out what decisions to make. For example, combinatorial optimization is a powerful tool used widely for decision-making in operations research. See [70] for a survey of how ML can be employed to help solve combinatorial optimization problems.

Tools from the field of multi-criteria decision-making can also help policymakers manage tradeoffs between different policies by reconciling competing objectives and minimizing negative sideeffects; in particular, in cases where policy objectives and constraints can be mathematically formalized, multi-objective optimization can provide a pragmatic approach to making decisions. Here, a decision-maker would formulate their decision-making process as an optimization problem by combining multiple optimization objectives subject to physical or other types of constraints; the goal is to then find a solution (or set of solutions) that is Pareto-optimal with respect to all of the objective functions. However, finding these solutions is often computationally expensive. Practitioners have applied bio-inspired algorithms such as particle swarm, genetic, or evolutionary algorithms to search for or compute Pareto-optimal solutions that satisfy the constraints. This 
approach has been applied in a number of climate change-related fields, including energy and infrastructure planning [38, 325, 525, 635, 732, 856], industry [122, 334], land use [462, 809], and more $[127,317,539,761]$. Previous work has also employed parallel surrogate search, assisted by ML, to efficiently solve multi-objective optimization problems [11]. Optimization algorithms which have been successful in the context of hyperparameter tuning (e.g., Bayesian optimization [726, 745]) or guided search algorithms (e.g., tree search algorithms [738]) could also potentially be applied to this problem.

Evaluating policy effects.

High Leverage When creating new policies, decision-makers may wish to understand previous policies (e.g., from other jurisdictions) and how these policies performed. ML can help analyze previous policy actions automatically and at scale by improving computational text analysis. In particular, natural language processing methods are already used in the field of political science to analyze political texts and legislation [307]; these approaches could be promising for systematically studying climate change policies. Causal inference techniques can also help assess the effect of a particular policy or climate-related event from observed outcomes. ML can play a role in causal inference [41, 340, 619], including in the context of policy problems [40, 453] and in climate-relevant scenarios such as estimating the effects of temperature on human mortality [356] and the effects of World Bank projects on vegetative cover [883].

\subsection{Designing Markets}

In economics, GHG emissions can be seen as a negative externality: while a changing climate results in a cost for society, this cost is often not reflected in the market price of goods or services that cause GHG emissions. This is problematic, since organizations and individuals making decisions solely on the basis of market prices will tend to favor cheaper goods, even if those goods emit a large amount of GHGs. Market-based tools ${ }^{33}$ such as cap-and-trade aim to enforce prices reflecting the societal cost of GHGs and thus encourage socially beneficial behavior through market forces. ML can help in understanding the impacts of market instruments; assessing their effectiveness at reducing emissions; and supporting a swift, effective and fair implementation. ${ }^{34}$

Predicting carbon prices.

There are several approaches to pricing GHG emissions. Carbon taxes and quotas aim to influence the behavior of organizations by shaping supply and demand within an existing market. By contrast, cap-and-trade approaches such as those within the European Union involve a completely new market, an Emissions Trading Scheme, within which companies can buy and sell a limited number of GHG emissions permits. Prices within such cap-and-trade markets are highly sensitive to control elements such as the number of permits released at a given time. ML can be used to analyze prices within these markets, for example by predicting prices via supervised learning [769, 834, 890, 892] or analyzing the main drivers of prices via hierarchical clustering [891].

\section{Non-carbon markets.}

Market design can influence GHG emissions even in settings where such emissions are not directly penalized. For instance, dynamic pricing in electricity markets-varying the price of electricity to consumers based on, e.g., how much wind power is available-can shape demand for low-carbon energy sources (see Section 2.1.1). Following seminal research on modeling pricing in markets as a bandit problem [700], many works have applied bandit and other RL algorithms to determine

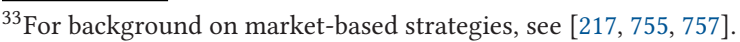

${ }^{34}$ For a review on ML for energy economics and finance, see [283].
} 
prices or other market values. For example, RL has been applied to predict bids [654] and market power [572] in electricity markets, and to set dynamic prices in more general settings [510]. ML can also help solve auctions in supply chains [708].

Assessing market effects.

When designing market-based strategies, it is necessary to understand how effectively each strategy will reduce emissions, as well as how the underlying socio-technical system may be affected. Studies have considered effects of carbon pricing on economic growth and energy intensity [233, 234], or on electricity prices [569]. Effects of pricing mechanisms can also be indirect, as companies' strategic decisions can have longer-term effects. ML can be useful in analyzing these effects. For example, self-organizing maps have been used to analyze how R\&D investment in green technologies changes in response to fuel prices [55], while a game theoretical framework using neural networks has been used to study the optimal production strategies for companies under carbon quotas [884].

To ensure that market-based strategies are effective and equitable, it is important to understand their distributional effects, as certain social groups or classes of stakeholders may be affected more than others. For example, a flat carbon tax on gasoline will have a larger effect on lower-income populations, as fuel expenses are a bigger share of their total budget. Here, clustering can help identify permit allocation schemes that maximize social welfare [855], and supervised learning has been used to predict winners and losers from changing electricity tariff schemes [302]. Hedonic pricing can also help identify how much different consumers may be willing to pay for a environmental good or a service, which is a noisy measure for the monetary value of that good or service; these values are typically inferred using regression or ML techniques on historical market data $[175,594,613,629]$. It is also important to analyze which organizations or individuals can actually participate in a given market. For example, carbon markets can be more flexible if viable offsets exist, including those offered by landowners who sequester carbon through forest conservation and management; ML has been used to examine the factors influencing the financial viability of such projects [425].

\subsection{Discussion}

The complexity, scale, and fundamental uncertainty inherent in the problems of climate change can pose challenges for collective decision-making. ML can help supplement existing mathematical frameworks that are employed to alleviate some of these challenges, including agent-based models, IAMs, multi-objective optimization, and market design tools. Interpretable and fair ML techniques may be of particular importance in this context, as they may enable decision-makers to more effectively and equitably employ insights from ML models. While these quantitative assessment tools can provide useful input to the decision-making process, it is worth noting that decisions regarding climate change may ultimately depend on qualitative discussions around norms, values, or equity considerations that may not be captured in quantitative models.

\section{EDUCATION}

BY ALEXANDRA LUCCIONI

Access to quality education is a key part of sustainable development, with significant benefits for climate and society at large. Education contributes to improving quality of life, helps individuals make informed decisions, and trains the next generation of innovators. Education is also paramount in helping people across societies understand and address the causes and consequences of climate change and provides the skills and tools necessary for adapting to its impacts. For instance, education can both improve the resilience of communities, particularly in developing countries that will be disproportionately affected by climate change [801], and empower individuals, 
especially from developed countries, to adopt more sustainable lifestyles [244]. As climate change itself may diminish educational outcomes for some populations, due to its negative effects on agricultural productivity and household income [666, 667], this makes providing high-quality educational interventions globally all the more important.

AI in Education.

Long-term

There are a number of ways that AI and ML can contribute to education and teaching-for instance, by improving access to educational opportunities, helping personalize the teaching process, and stepping in when teachers have limited time. The field of Artificial Intelligence in EDucation (AIED) has existed for over 30 years, and until recently relied on explicitly modeling content, learners, and tutoring strategies based on psychological theories of learning. However, AIED is increasingly incorporating data-driven insights derived from ML techniques.

One important area of AIED research has been Intelligent Tutoring Systems (ITSs), which can adapt their behavior in real time according to the needs of individuals or to support collaborative learning [124]. While ITSs have traditionally been defined and constructed by hand, recent approaches have applied ML techniques such as multi-armed bandit techniques to adaptively personalize sequences of learning activities [135], LSTMs to generate questions to evaluate language comprehension [205], and RL to improve the strategies used within the ITS [365, 441]. However, there remains much work to be done to bridge the performance gap between digital and human tutors, and ML-based approaches have an important role to play in this endeavor-for example, via natural language processing techniques for creating conversational agents [295], learner analytics for classifying student profiles, [695], and adaptive learning approaches to propose relevant educational activities and exercises [774]. ${ }^{35}$

While ITSs generally focus on individualized or small-group instruction, AIED can also help provide tools that improve educational outcomes at scale for larger groups of learners. For instance, scalable, adaptive online courses could give hundreds of thousands of learners access to learning resources that they would not usually have in their local educational facilities [694]. Furthermore, giving teachers guidance derived from computational teaching algorithms or heuristics could help them design better educational curricula and improve student learning outcomes [172]. In this context, AIED applications can be used either as a standalone tool for independent learners or as an educational resource that frees up teachers to have more one-on-one time with students. Key considerations for creating AIED tools that can be applied across the globe include adapting to local technological and cultural needs, addressing barriers such as access to electricity and internet [430, 431], and taking into account students' computing skills, language, and culture [106, 590].

\section{Learning about climate.}

Research has shown that educational activities centered on climate change and carbon footprints can engage learners in understanding the connection between personal and collective actions and their impact on global climate, and can enable individuals to make climate-friendly lifestyle choices such as reducing energy use [142]. There have also been proposals for interactive websites explaining climate science as well as educational interventions focusing on local and actionable aspects of sustainable development [22]. In these contexts, ML can help create personalized educational tools, for instance by generating images of potential future impacts of extreme weather events based on a learner's address [716] or by anchoring an individual's learning experience in a digital replica of their real-life location and allowing them to explore the way that climate change will impact a specific location [28].

\footnotetext{
${ }^{35}$ For further background on this area, see $[395,582,630]$.
} 


\section{FINANCE}

BY ALEXANDRA LUCCIONI

The rise and fall of financial markets is linked to many events, both sporadic (e.g., the 2008 global financial crisis) and cyclical (e.g., the price of gas over the years), with profits and losses that can be measured in the billions of dollars and can have global consequences. Climate change poses a substantial financial risks to global assets measured in the trillions of dollars [185], and it is hard to forecast where, how, or when climate change will impact the stock price of a given company, or even the debt of an entire nation. While financial analysts and investors focus on pricing risk and forecasting potential earnings, the majority of the current financial system is based on quarterly or yearly performance. This fails to incentivize the prediction of medium or long-term risks, which include most climate change-related exposures such as physical impacts on assets or distribution chains, legislative impacts on profit generation, and indirect market consequences such as supply and demand. ${ }^{36}$

\section{Climate investment.}

Climate investment, the current dominant approach in climate finance, involves investing money in low-carbon assets [229]. The dominant ways in which major financial institutions take this approach are by creating "green" financial indexes that focus on low-carbon energy, clean technology, and/or environmental services [182] or by designing carbon-neutral investment portfolios that remove or under-weight companies with relatively high carbon footprints [284]. This investment strategy is creating major shifts in certain sectors of the market (e.g., utilities and energy) towards renewable energy alternatives, which are seen as having a greater growth potential than traditional energy sources such as oil and gas [72]. While this approach currently does not utilize ML directly, we see the potential in applying deep learning both for portfolio selection (based on features of the stocks involved) and investment timing (using historical patterns to predict future demand), to maximize both the impact and scope of climate investment strategies.

Climate analytics.

High Leverage

The other main approach to climate finance is climate analytics, which aims to predict the financial effects of climate change, and is still gaining momentum in the mainstream financial community [229]. Since this is a predictive approach to addressing climate change from a financial perspective, it is one where ML can potentially have greater impact. Climate analytics involves analyzing investment portfolios, funds, and companies in order to pinpoint areas with heightened risk due to climate change, such as timber companies that could be bankrupted by wildfires or water extraction initiatives that could see their sources polluted by shifting landscapes. Approaches used in this field include: natural language processing techniques for identifying climate risks and investment opportunities in disclosures made by companies [254, 442, 498, 752] as well as for analyzing the evolution of climate coverage in the media to dynamically hedge climate change risk [221]; econometric approaches for developing arbitrage strategies that take advantage of the carbon risk factor in financial markets [26]; and ML approaches for forecasting the price of carbon in emission exchanges ${ }^{37}$ [887, 889].

To date, the field of climate finance has been largely neglected within the larger scope of financial research and analysis. This leaves many directions for improvement, such as (1) improving existing traditional portfolio optimization approaches; (2) in-depth modeling of variables linked to climate risk; (3) designing a statistical climate factor that can be used to project the variation of stock prices

\footnotetext{
${ }^{36}$ For further reading regarding the impact of climate change on financial markets, see [61, 86, 108].

${ }^{37}$ Carbon pricing, e.g., via $\mathrm{CO}_{2}$ cap-and-trade or a carbon tax, is a commonly-suggested policy approach for getting firms to price future climate change impacts into their financial calculations. For an introduction to these topics, see [632] and also Section 12.3
} 
given a compound set of events; and (4) identifying direct and indirect climate risk exposure in annual company reports. ML plays a central role in these strategies, and can be a powerful tool in leveraging the financial sector to mitigate climate change and in reducing the financial impacts of climate change on society.

\section{CONCLUSION}

ML, like any technology, does not always make the world a better place-but it can. In the fight against climate change, ML has significant contributions to offer across domain areas. ML can enable automatic monitoring through remote sensing (e.g., by pinpointing deforestation, gathering data on buildings, and assessing damage after disasters). It can accelerate the process of scientific discovery (e.g., by suggesting new materials for batteries, construction, and carbon capture). ML can optimize systems to improve efficiency (e.g., by consolidating freight, designing carbon markets, and reducing food waste). And it can accelerate computationally expensive physical simulations through hybrid modeling (e.g., climate models and energy scheduling models). These and other cross-cutting themes are shown in Table 2. We emphasize that in each application, ML is only one part of the solution; it is a tool that enables other tools across fields.

Applying ML to tackle climate change has the potential both to benefit society and to advance the field of ML. Many of the problems we have discussed here highlight cutting-edge areas of ML, such as interpretability, causality, and uncertainty quantification. Moreover, meaningful action on climate problems requires dialogue with fields within and outside computer science and can lead to interdisciplinary methodological innovations, such as improved physics-constrained ML techniques.

The nature of climate-relevant data poses challenges and opportunities. For many of the applications we identify, data can be proprietary or include sensitive personal information. Where datasets exist, they may not be organized with a specific task in mind, unlike typical ML benchmarks that have a clear objective. Datasets may include information from heterogeneous sources, which must be integrated using domain knowledge. Moreover, the available data may not be representative of global use cases. For example, forecasts of electricity demand based on a dataset from the US will not necessarily generalize to India, where patterns of demand may be different. Tools from transfer learning and domain adaptation will likely prove essential in low-data settings. For some tasks, it may also be feasible to augment learning with carefully simulated data. Of course, the best option if possible is always more real data; we strongly encourage public and private entities to release datasets and to solicit involvement from the ML community.

For those looking to use ML to help tackle climate change, we provide further resources via the Climate Change AI initiative (www.climatechange.ai), and we offer the following roadmap:

- Learn. Identify how your skills may be useful-we hope this article is a starting point. Remember that often the most impactful work lies in solving well-defined, domain-specific bottlenecks, and is not always flashy.

- Collaborate. Find collaborators, who may be researchers, entrepreneurs, established companies, or policy makers. Every domain discussed here has experts who understand its opportunities and pitfalls, even if they are not experts in ML.

- Listen. Listen to what your collaborators and other stakeholders say is needed for addressing the problem effectively. Keep in mind that complex methodologies are not always needed.

- Deploy. Work with deployment partners to ensure a pathway to impact for your work, and incorporate deployment-related considerations during development.

We call upon the ML community to use its skills as part of the global effort against climate change. 
Table 2. Cross-Cutting Objectives that are Relevant to Many Climate Change Domains

\begin{tabular}{|c|c|c|c|c|c|c|c|c|c|}
\hline & & 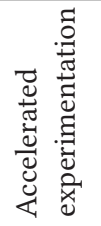 & 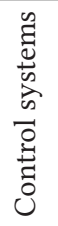 & 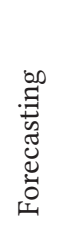 & 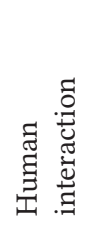 & 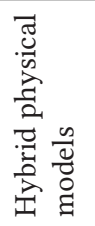 & 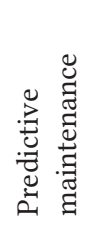 & 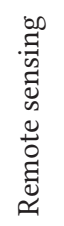 & 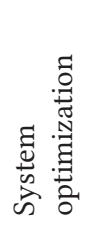 \\
\hline \multirow{25}{*}{ 苛 } & Electricity systems & & & & & & & & \\
\hline & Enabling low-carbon electricity & - & - & • & & - & • & - & - \\
\hline & Reducing current-system impacts & & & - & & & - & - & \\
\hline & Ensuring global impact & & & - & & - & & - & \\
\hline & Transportation & & & & & & & & \\
\hline & Reducing transport activity & - & - & - & & & & - & - \\
\hline & Improving vehicle efficiency & - & - & & & & & & - \\
\hline & Alternative fuels \& electrification & - & - & - & & & & & - \\
\hline & Modal shift & & - & - & - & & - & - & - \\
\hline & Buildings and cities & & & & & & & & \\
\hline & Optimizing buildings & & - & - & & - & - & & - \\
\hline & Urban planning & & & & & & & $\bullet$ & \\
\hline & The future of cities & & & & & & & & - \\
\hline & Industry & & & & & & & & \\
\hline & Optimizing supply chains & & - & - & & & & & - \\
\hline & Improving materials & - & & & & & & & \\
\hline & Production \& energy & & - & & & & - & & - \\
\hline & Farms \& forests & & & & & & & & \\
\hline & Remote sensing of emissions & & & & & & & - & \\
\hline & Precision agriculture & & $\bullet$ & $\bullet$ & & & & $\bullet$ & \\
\hline & Monitoring peatlands & & & & & & & • & \\
\hline & Managing forests & & - & - & & & & - & \\
\hline & Carbon dioxide removal & & & & & & & & \\
\hline & Direct air capture & - & & & & - & & & \\
\hline & Sequestering $\mathrm{CO}_{2}$ & & & & & - & & & \\
\hline \multirow{12}{*}{ } & Climate prediction & & & & & & & & \\
\hline & Uniting data, ML \& climate science & & & $\bullet$ & & $\bullet$ & & $\bullet$ & \\
\hline & Forecasting extreme events & & & • & & - & & • & \\
\hline & Societal impacts & & & & & & & & \\
\hline & Ecology & & & & & & & - & \\
\hline & Infrastructure & & & & & & - & & - \\
\hline & Social systems & & & - & - & & & - & - \\
\hline & Crisis & & & - & & & & - & \\
\hline & Solar geoengineering & & & & & & & & \\
\hline & Understanding \& improving aerosols & & - & & & - & & & \\
\hline & Engineering a control system & & $\bullet$ & & & $\bullet$ & & & \\
\hline & Modeling impacts & & & & & - & & & \\
\hline \multirow{9}{*}{ 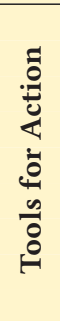 } & Individual action & & & & & & & & \\
\hline & Understanding personal footprint & & & - & - & & & & \\
\hline & Facilitating behavior change & & & & - & & & & \\
\hline & Collective decisions & & & & & & & & \\
\hline & Modeling social interactions & & & & $\bullet$ & & & & \\
\hline & Informing policy & & & & - & & & & \\
\hline & Designing markets & & & - & & & & & - \\
\hline & Education & & & & - & & & & \\
\hline & Finance & & & - & - & & & & \\
\hline
\end{tabular}




\section{ACKNOWLEDGMENTS}

Electricity systems. We thank James Kelloway (National Grid ESO), Jack Kelly (Open Climate Fix), Zico Kolter (CMU), and Henry Richardson (WattTime) for their help and ideas in shaping this section. We also thank Samuel Buteau (Dalhousie University) and Marc Cormier (Dalhousie University) for their inputs on accelerated science and battery storage technologies; Julian Kates-Harbeck (Harvard) and Melrose Roderick (CMU) for their extensive inputs and ideas on nuclear fusion; and Alasdair Bruce (formerly National Grid ESO) for inputs on emissions factor forecasting and automated dispatch. Finally, we thank Lea Boche (EPRI), Carl Elkin (DeepMind), Jim Gao (DeepMind), Muhammad Hasan (DeepMind), Guannan He (CMU), Jeremy Keen (CMU), Zico Kolter (CMU), Luke Lavin (CMU), Sanam Mirzazad (EPRI), David Pfau (DeepMind), Crystal Qian (DeepMind), Juliet Rothenberg (DeepMind), Sims Witherspoon (DeepMind), and Matt Wytock (Gridmatic, Inc.) for helpful comments and feedback.

Transportation. We are grateful for advice from Alan T. Jenn (UC Davis) and Prithvi S. Acharya $(\mathrm{CMU})$ on electric vehicles, Alexandre Jacquillat (CMU) on decarbonizing aviation, Michael Whiston (CMU) on hydrogen fuel cells, Evan Sherwin (CMU) on alternative fuels, and Samuel Buteau (Dalhousie University) on batteries.

Buildings and Cities. We thank Érika Mata (IVL - Swedish Environmental Research Institute, IPCC Lead Author Buildings section), Duccio Piovani (nam.R), Hari Prasanna Das (UC Berkeley), and Jack Kelly (Open Climate Fix) for feedback and ideas.

Industry. We appreciate all the constructive feedback from Angela Acocella (MIT), Kevin McCloskey (Google), and Bill Tubbs (University of British Columbia), and we are grateful to Kipp Bradford (Yale) for his recommendations around embodied energy and refrigeration. Thanks to Allie Schwertner (Rockwell Automation), Greg Kochanski (Google), and Paul Weaver (Abstract) for their suggestions around optimizing industrial processes for low-carbon energy.

Farms \& Forests. We would like to give thanks to David Marvin (Salo), Remi Charpentier (Tesselo), and David Dao (ETH Zürich) for their input on remote sensing for land use. Max Nova (SilviaTerra) provided insight on forestry, Mark Crowley (University of British Columbia) on forest fire management, Benjamin Deleener (ChrysaLabs) on precision agriculture, and Lindsay Brin (Element AI) on soil chemistry.

Climate prediction. We thank Ghaleb Abdulla (LLNL), Ben Kravitz (PNNL), and David John Gagne II (UCAR) for enlightening conversations; Goodwin Gibbins (Imperial College London) and Ben Kravitz (PNNL) for detailed editing and feedback; and Claire Monteleoni (CU Boulder) and Prabhat (LBL) for feedback which improved the quality of this manuscript.

Societal impacts. We thank Loubna Benabbou (UQAR), Mike Schäfer (University of Zurich), Andrea Garcia Tapia (Stevens Tech), Slava Jankin Mikhaylov (Hertie School Berlin), and Sarah $\mathrm{M}$. Fletcher (MIT) for valuable conversations on the social aspects of climate change.

Solar geoengineering. We thank David Keith (Harvard), Peter Irvine (Harvard), Zhen Dai (Harvard), Colleen Golja (Harvard), Ross Boczar (UC Berkeley), Jon Proctor (UC Berkeley), Ben Kravitz (Indiana University), Andrew Lockley (University College London), Trude Storelvmo (University of Oslo), and Simon Gruber (University of Oslo) for help and useful feedback.

Individual action. We thank Priyanka deSouza (MIT), Olivier Corradi (Tomorrow), Jack Kelly (Open Climate Fix), Ioana Marinescu (UPenn), and Aven Satre-Meloy (Oxford). 
Collective decisions. We thank Sebastian Sewerin (ETH Zürich), D. Cale Reeves (UT Austin), and Rahul Ladhania (UPenn).

Education. We appreciated the constructive feedback received by Jacqueline Bourdeau (TÉLUQ University), who gave us valuable insights regarding the field of AIED.

Finance. We thank Himanshu Gupta (ClimateAI) and Bjarne Steffen (ETH Zürich) for constructive discussions and the valuable feedback.

We also thank the anonymous reviewers for their helpful comments.

\section{REFERENCES}

[1] Hany Abdelrahman, Felix Berkenkamp, Jan Poland, and Andreas Krause. 2016. Bayesian optimization for maximum power point tracking in photovoltaic power plants. In 2016 European Control Conference (ECC'16). IEEE, 2078-2083.

[2] Majid A. Abdullah, A. H. M. Yatim, Chee Wei Tan, and Rahman Saidur. 2012. A review of maximum power point tracking algorithms for wind energy systems. Renewable and Sustainable Energy Reviews 16, 5 (2012), 3220-3227.

[3] David Abel, Edward C. Williams, Stephen Brawner, Emily Reif, and Michael L. Littman. 2018. Bandit-based solar panel control. In 32nd AAAI Conference on Artificial Intelligence.

[4] Zakia Afroz, G. M. Shafiullah, Tania Urmee, and Gary Higgins. 2018. Modeling techniques used in building HVAC control systems: A review. Renewable and Sustainable Energy Reviews 83 (2018), 64-84.

[5] Muhammad Aftab, Chien Chen, Chi-Kin Chau, and Talal Rahwan. 2017. Automatic HVAC control with real-time occupancy recognition and simulation-guided model predictive control in low-cost embedded system. Energy and Buildings 154 (2017), 141-156.

[6] International Energy Agency. 2011. Biofuels for Transport. OECD Publishing, Paris. https://doi.org/10.1787/ 9789264118461-en

[7] Arun Agrawal and Nicolas Perrin. 2009. Climate adaptation, local institutions and rural livelihoods. In Adapting to Climate Change: Thresholds, Values, Governance. Cambridge University Press, Cambridge. 350-367.

[8] Ejaz Ahmed, Ibrar Yaqoob, Arif Ahmed, Abdullah Gani, Muhammad Imran, and Sghaier Guizani. 2016. Green industrial networking: Recent advances, taxonomy, and open research challenges. IEEE Communications Magazine 54, 10 (2016), 38-45.

[9] Razin Ahmed, V. Sreeram, Y. Mishra, and M. D. Arif. 2020. A review and evaluation of the state-of-the-art in PV solar power forecasting: Techniques and optimization. Renewable and Sustainable Energy Reviews 124 (2020), 109792.

[10] Hashem Akbari, H. Damon Matthews, and Donny Seto. 2012. The long-term effect of increasing the albedo of urban areas. Environmental Research Letters 7, 2 (2012), 024004

[11] Taimoor Akhtar and Christine A. Shoemaker. 2019. Efficient multi-objective optimization through population-based parallel surrogate search. arXiv preprint arXiv:1903.02167 (2019).

[12] A. Okay Akyuz, Mitat Uysal, Berna Atak Bulbul, and M. Ozan Uysal. 2017. Ensemble approach for time series analysis in demand forecasting: Ensemble learning. In 2017 IEEE International Conference on INnovations in Intelligent SysTems and Applications (INISTA'17). IEEE, 7-12.

[13] Heidi J. Albers, Kim Meyer Hall, Katherine D. Lee, Majid Alkaee Taleghan, and Thomas G. Dietterich. 2018. The role of restoration and key ecological invasion mechanisms in optimal spatial-dynamic management of invasive species. Ecological Economics 151 (2018), 44-54.

[14] Adrian Albert and Mehdi Maasoumy. 2016. Predictive segmentation of energy consumers. Applied Energy 177 (2016), 435-448.

[15] Ahmed M. Ali and Dirk Söffker. 2018. Towards optimal power management of hybrid electric vehicles in real-time: A review on methods, challenges, and state-of-the-art solutions. Energies 11, 3 (2018), 476.

[16] Hunt Allcott. 2011. Social norms and energy conservation. Journal of Public Economics 95, 9-10 (2011), $1082-1095$.

[17] Hunt Allcott and Todd Rogers. 2014. The short-run and long-run effects of behavioral interventions: Experimental evidence from energy conservation. American Economic Review 104, 10 (2014), 3003-37.

[18] Mehmet Altinkaya and Metin Zontul. 2013. Urban bus arrival time prediction: A review of computational models. International fournal of Recent Technology and Engineering 2, 4 (2013), 164-169.

[19] Ahmad Alzahrani, Pourya Shamsi, Cihan Dagli, and Mehdi Ferdowsi. 2017. Solar irradiance forecasting using deep neural networks. Procedia Computer Science 114 (2017), 304-313.

[20] Kadir Amasyali and Nora M. El-Gohary. 2018. A review of data-driven building energy consumption prediction studies. Renewable and Sustainable Energy Reviews 81, 1 (2018), 1192-1205. 
[21] Brandon Amos, Ivan Jimenez, Jacob Sacks, Byron Boots, and J. Zico Kolter. 2018. Differentiable MPC for end-to-end planning and control. In Advances in Neural Information Processing Systems. 8289-8300.

[22] Allison Anderson. 2012. Climate change education for mitigation and adaptation. fournal of Education for Sustainable Development 6, 2 (2012), 191-206.

[23] Gemma Anderson and Donald D. Lucas. 2018. Machine learning predictions of a multiresolution climate model ensemble. Geophysical Research Letters 45, 9 (2018), 4273-4280.

[24] James Anderson, Fengyu Zhou, and Steven H. Low. 2018. Disaggregation for networked power systems. In 2018 Power Systems Computation Conference (PSCC'18). IEEE, 1-7.

[25] Kirstin Anderson-Hall, Brandon Bordenkircher, Riley O’Neil, and Smith C. Scott. 2019. Governing Micro-Mobility: A Nation wide Assessment of Electric Scooter Regulations. Technical Report.

[26] Mats Andersson, Patrick Bolton, and Frédéric Samama. 2016. Hedging climate risk. Financial Analysts fournal 72, 3 (2016), 13-32.

[27] Mathew Aneke and Meihong Wang. 2016. Energy storage technologies and real life applications-A state of the art review. Applied Energy 179 (2016), 350-377.

[28] Jeannette Angel, Alicia LaValle, Deepti Mathew Iype, Stephen Sheppard, and Aleksandra Dulic. 2015. Future delta 2.0 an experiential learning context for a serious game about local climate change. In SIGGRAPH Asia 2015 Symposium on Education. ACM, 12.

[29] Roger Angel. 2006. Feasibility of cooling the Earth with a cloud of small spacecraft near the inner Lagrange point (L1). Proceedings of the National Academy of Sciences 103, 46 (2006), 17184-17189.

[30] Ioannis Antonopoulos, Valentin Robu, Benoit Couraud, Desen Kirli, Sonam Norbu, Aristides Kiprakis, David Flynn, Sergio Elizondo-Gonzalez, and Steve Wattam. 2020. Artificial intelligence and machine learning approaches to energy demand-side response: A systematic review. Renewable and Sustainable Energy Reviews 130 (2020), 109899.

[31] Mauricio Araya-Polo, Joseph Jennings, Amir Adler, and Taylor Dahlke. 2018. Deep-learning tomography. The Leading Edge 37, 1 (2018), 58-66.

[32] David Archer and Stefan Rahmstorf. 2010. The Climate Crisis: An Introductory Guide to Climate Change. Cambridge University Press.

[33] K. Carrie Armel, Abhay Gupta, Gireesh Shrimali, and Adrian Albert. 2013. Is disaggregation the holy grail of energy efficiency? The case of electricity. Energy Policy 52 (2013), 213-234.

[34] Peter Arnfalk, Ulf Pilerot, Per Schillander, and Pontus Grönvall. 2016. Green IT in practice: Virtual meetings in Swedish public agencies. fournal of Cleaner Production 123 (2016), 101-112.

[35] J. F. Artaud, V. Basiuk, F. Imbeaux, Martin Schneider, J. Garcia, G. Giruzzi, P. Huynh, T. Aniel, F. Albajar, J. M. Ané, A. Bécoulet, C. Bourdelle, A. Casati, L. Colas, J. Decker, R. Dumont, L. G. Eriksson, X. Garbet, R. Guirlet, P. Hertout, G. T. Hoang, W. Houlberg, G. Huysmans, E. Joffrin, S. H. Kim, F. Köchl, J. Lister, X. Litaudon, P. Maget, R. Masset, B. Pégourié, Y. Peysson, P. Thomas, E. Tsitrone, and F. Turco. 2010. The CRONOS suite of codes for integrated tokamak modelling. Nuclear Fusion 50, 4 (2010), 043001.

[36] W. Brian Arthur. 1989. Competing technologies, increasing returns, and lock-in by historical events. The Economic Journal 99, 394 (1989), 116-131.

[37] Solomon Assefa. 2018. Hello Tractor Pilot Agriculture Digital Wallet based on AI and Blockchain. Retrieved from https://www.ibm.com/blogs/research/2018/12/hello-tractor/.

[38] Mohammad Saeid Atabaki and Vahid Aryanpur. 2018. Multi-objective optimization for sustainable development of the power sector: An economic, environmental, and social analysis of Iran. Energy 161 (2018), 493-507.

[39] Muhammad Ateeq, Farruh Ishmanov, Muhammad Khalil Afzal, and Muhammad Naeem. 2019. Multi-parametric analysis of reliability and energy consumption in IoT: A deep learning approach. Sensors 19, 2 (2019), 309.

[40] Susan Athey. 2017. Beyond prediction: Using big data for policy problems. Science 355, 6324 (2017), 483-485.

[41] Susan Athey and Guido W. Imbens. 2019. Machine learning methods that economists should know about. Annual Review of Economics 11, 1 (2019), 685-725.

[42] Peter M. Attia, Aditya Grover, Norman Jin, Kristen A. Severson, Todor M. Markov, Yang-Hung Liao, Michael H. Chen, Bryan Cheong, Nicholas Perkins, Zi Yang, Patrick K. Herring, Muratahan Aykol, Stephen J. Harris, Richard D. Braatz, Stefano Ermon, and William C. Chueh. 2020. Closed-loop optimization of fast-charging protocols for batteries with machine learning. Nature 578, 7795 (2020), 397-402.

[43] Rockwell Automation. 2014. AkzoNobel Powder Coatings saves over 15,000 euros per month thanks to advanced energy monitoring solution from Rockwell Automation. Retrived from https://iterature.rockwellautomation.com/ idc/groups/literature/documents/ap/energy-ap009_-en-p.pdf.

[44] Jonn Axsen and Benjamin K. Sovacool. 2019. The roles of users in electric, shared and automated mobility transitions. Transportation Research Part D: Transport and Environment 71 (2019), 1-21.

[45] Inês M. L. Azevedo. 2014. Consumer end-use energy efficiency and rebound effects. Annual Review of Environment and Resources 39 (2014), 393-418. 
[46] H. M. Abdul Aziz and Satish V. Ukkusuri. 2018. A novel approach to estimate emissions from large transportation networks: Hierarchical clustering-based link-driving-schedules for EPA-MOVES using dynamic time warping measures. International fournal of Sustainable Transportation 12, 3 (2018), 192-204.

[47] Oytun Babacan, Ahmed Abdulla, Ryan Hanna, Jan Kleissl, and David G. Victor. 2018. Unintended effects of residential energy storage on emissions from the electric power system. Environmental Science \& Technology 52, 22 (2018), 1360013608.

[48] A. G. S. J. Baccini, S. J. Goetz, W. S. Walker, N. T. Laporte, M. Sun, D. Sulla-Menashe, J. Hackler, P. S. A. Beck, R. Dubayah, M. A. Friedl, S. Samanta, and R. A. Houghton. 2012. Estimated carbon dioxide emissions from tropical deforestation improved by carbon-density maps. Nature climate change 2, 3 (2012), 182.

[49] Junwen Bai, Yexiang Xue, Johan Bjorck, Ronan Le Bras, Brendan Rappazzo, Richard Bernstein, Santosh K. Suram, Robert Bruce van Dover, John M. Gregoire, and Carla P. Gomes. 2018. Phase mapper: Accelerating materials discovery with AI. AI Magazine 39, 1 (2018), 15-26.

[50] Xuemei Bai, Richard J. Dawson, Diana Ürge-Vorsatz, Gian C. Delgado, Aliyu Salisu Barau, Shobhakar Dhakal, David Dodman, Lykke Leonardsen, Valérie Masson-Delmotte, Debra C. Roberts, and Seth Schultz. 2018. Six research priorities for cities and climate change. Nature 555, 7964 (2018), 23-25.

[51] Zubair A. Baig. 2011. On the use of pattern matching for rapid anomaly detection in smart grid infrastructures. In 2011 IEEE International Conference on Smart Grid Communications (SmartGridComm'11). IEEE, 214-219.

[52] Kyri Baker. 2019. Learning warm-start points for AC optimal power flow. In 2019 IEEE 29th International Workshop on Machine Learning for Signal Processing (MLSP'19). IEEE, 1-6.

[53] E. A. Baltz, E. Trask, M. Binderbauer, M. Dikovsky, H. Gota, R. Mendoza, J. C. Platt, and P. F. Riley. 2017. Achievement of sustained net plasma heating in a fusion experiment with the optometrist algorithm. Scientific Reports 7, 1 (2017) 6425.

[54] Kirk Bansak, Jeremy Ferwerda, Jens Hainmueller, Andrea Dillon, Dominik Hangartner, Duncan Lawrence, and Jeremy Weinstein. 2018. Improving refugee integration through data-driven algorithmic assignment. Science 359, 6373 (2018), 325-329.

[55] Nicolò Barbieri. 2016. Fuel prices and the invention crowding out effect: Releasing the automotive industry from its dependence on fossil fuel. Technological Forecasting and Social Change 111 (2016), 222-234.

[56] William Barbour, Juan Carlos Martinez Mori, Shankara Kuppa, and Daniel B. Work. 2018. Prediction of arrival times of freight traffic on US railroads using support vector regression. Transportation Research Part C: Emerging Technologies 93 (2018), 211-227.

[57] Justin E. Barton, William P. Wehner, Eugenio Schuster, Federico Felici, and Olivier Sauter. 2015. Simultaneous closedloop control of the current profile and the electron temperature profile in the TCV tokamak. In 2015 American Control Conference (ACC'15). IEEE, 3316-3321.

[58] Kelsey Barton-Henry, Leonie Wenz, and Anders Levermann. 2021. Decay radius of climate decision for solar panels in the city of Fresno, USA. Scientific Reports 11, 1 (2021), 1-9.

[59] Favyen Bastani, Songtao He, Sofiane Abbar, Mohammad Alizadeh, Hari Balakrishnan, Sanjay Chawla, and Sam Madden. 2018. Machine-assisted map editing. In 26th ACM SIGSPATIAL International Conference on Advances in Geographic Information Systems. ACM, 23-32.

[60] Jean-Francois Bastin, Yelena Finegold, Claude Garcia, Danilo Mollicone, Marcelo Rezende, Devin Routh, Constantin M. Zohner, and Thomas W. Crowther. 2019. The global tree restoration potential. Science 365, 6448 (2019), 76-79.

[61] Stefano Battiston, Antoine Mandel, Irene Monasterolo, Franziska Schütze, and Gabriele Visentin. 2017. A climate stress-test of the financial system. Nature Climate Change 7, 4 (2017), 283.

[62] Ivanna Baturynska, Oleksandr Semeniuta, and Kristian Martinsen. 2018. Optimization of process parameters for powder bed fusion additive manufacturing by combination of machine learning and finite element method: A conceptual framework. Procedia CIRP 67 (2018), 227-232.

[63] Christian Beckel, Leyna Sadamori, and Silvia Santini. 2013. Automatic socio-economic classification of households using electricity consumption data. In 4th International Conference on Future Energy Systems. ACM, 75-86.

[64] Sara Beery, Yang Liu, Dan Morris, Jim Piavis, Ashish Kapoor, Markus Meister, and Pietro Perona. 2019. Synthetic examples improve generalization for rare classes. In IEEE Winter Conference on Applications of Computer Vision (WACV'20).

[65] Liam F. Beiser-McGrath and Thomas Bernauer. 2019. Commitment failures are unlikely to undermine public support for the Paris agreement. Nature Climate Change 9, 3 (2019), 248.

[66] Liam F. Beiser-McGrath and Robert A. Huber. 2018. Assessing the relative importance of psychological and demographic factors for predicting climate and environmental attitudes. Climatic Change 149, 3-4 (2018), 335-347.

[67] Wanda Bell, Lewis Ahron Kaufman, William Joseph Krajewski, John J. McGillicuddy, Paul Aloysius Scanlon, Jr., Abhijit Dey, Sharon Ameet Fanse, Giridhar Holenarsipur Nagaraj, Shyamli Rai, Sunitha Sundaramurthy, Gurpreet Chahil, Jeetendra Chandwani, Arham GuptaMangesh Ashok Karhadkar, Vincent Francis La Padula, Paul J. Murray, 
Himanshu Shailesh Shah, and Rasika Vartak. 2016. Systems and methods for automated data privacy compliance. US Patent No. 9,507,960.

[68] Asher Bender, Brett Whelan, and Salah Sukkarieh. 2019. Ladybird Cobbitty 2017 Brassica Dataset. The University of Sydney. https://doi.org/10.25910/5c941d0c8bccb

[69] Emily M. Bender, Timnit Gebru, Angelina McMillan-Major, and Shmargaret Shmitchell. 2021. On the dangers of stochastic parrots: Can language models be too big? In ACM Conference on Fairness, Accountability, and Transparency.

[70] Yoshua Bengio, Andrea Lodi, and Antoine Prouvost. 2021. Machine learning for combinatorial optimization: A methodological tour d'horizon. European fournal of Operational Research 290, 2 (2021), 405-421.

[71] Bettina Berendt. 2019. AI for the Common Good?! Pitfalls, challenges, and ethics pen-testing. Paladyn, fournal of Behavioral Robotics 10, 1 (2019), 44-65.

[72] Ariel Bergmann, Nick Hanley, and Robert Wright. 2006. Valuing the attributes of renewable energy investments. Energy Policy 34, 9 (2006), 1004-1014.

[73] Peter F. Bernath, Mahdi Yousefi, Eric Buzan, and Chris D. Boone. 2017. A near-global atmospheric distribution of N2O isotopologues. Geophysical Research Letters 44, 20 (2017), 10-735.

[74] Josep Ll. Berral, Íñigo Goiri, Ramón Nou, Ferran Julià, Jordi Guitart, Ricard Gavaldà, and Jordi Torres. 2010. Towards energy-aware scheduling in data centers using machine learning. In P1st International Conference on Energy-Efficient Computing and Networking (e-Energy'10). ACM, New York, NY, 215-224.

[75] Dimitris Bertsimas and Bartolomeo Stellato. 2019. Online mixed-integer optimization in milliseconds. Preprint arXiv:1907.02206 (2019).

[76] Biswarup Bhattacharya and Abhishek Sinha. 2017. Deep fault analysis and subset selection in solar power grids. Preprint arXiv:1711.02810 (2017).

[77] Filip Biljecki, Hugo Ledoux, and Jantien Stoter. 2017. Generating 3D city models without elevation data. Computers, Environment and Urban Systems 64 (2017), 1-18.

[78] Christopher M. Bishop. 2006. Pattern Recognition and Machine Learning. Springer.

[79] Maros Blaha, Christoph Vogel, Audrey Richard, Jan D. Wegner, Thomas Pock, and Konrad Schindler. 2016. Largescale semantic 3D reconstruction: An adaptive multi-resolution model for multi-class volumetric labeling. In IEEE Conference on Computer Vision and Pattern Recognition. 3176-3184.

[80] Blue River Technology. 2021. Blue River Technology. Retrieved from https://bluerivertechnology.com/.

[81] Bluefield Technologies. 2021. Bluefield Technologies. Retrieved from http://bluefield.co/.

[82] Joshua E. Blumenstock. 2012. Inferring patterns of internal migration from mobile phone call records: Evidence from Rwanda. Information Technology for Development 18, 2 (2012), 107-125.

[83] Erwan Bocher, Gwendall Petit, Jérémy Bernard, and Sylvain Palominos. 2018. A geoprocessing framework to compute urban indicators: The MApUCE tools chain. Urban Climate 24 (2018), 153-174.

[84] Ross Boczar, Nikolai Matni, and Benjamin Recht. 2018. Finite-data performance guarantees for the output-feedback control of an unknown system. In 2018 IEEE Conference on Decision and Control (CDC'18). IEEE, 2994-2999.

[85] Andrey Bogomolov, Bruno Lepri, Roberto Larcher, Fabrizio Antonelli, Fabio Pianesi, and Alex Pentland. 2016. Energy consumption prediction using people dynamics derived from cellular network data. EPf Data Science 5, 1 (2016), 13.

[86] Jean Boissinot, Doryane Huber, and Gildas Lame. 2016. Finance and Climate: The transition to a low-carbon and climate-resilient economy from a financial sector perspective. OECD fournal: Financial Market Trends 2015/ 1 (2016), $7-23$.

[87] Mariusz Bojarski, Davide Del Testa, Daniel Dworakowski, Bernhard Firner, Beat Flepp, Prasoon Goyal, Lawrence D. Jackel, Mathew Monfort, Urs Muller, Jiakai Zhang, Xin Zhang, Jake Zhao, and Karol Zieba. 2016. End to End Learning for Self-Driving Cars. arXiv preprint arXiv:1604.07316 (2016).

[88] Bryan Bollinger and Kenneth Gillingham. 2012. Peer effects in the diffusion of solar photovoltaic panels. Marketing Science 31, 6 (2012), 900-912

[89] Antoine Bordes, Xavier Glorot, Jason Weston, and Yoshua Bengio. 2014. A semantic matching energy function for learning with multi-relational data. Machine Learning 94, 2 (2014), 233-259.

[90] Christian Borgs, Ozan Candogan, Jennifer Chayes, Ilan Lobel, and Hamid Nazerzadeh. 2014. Optimal multiperiod pricing with service guarantees. Management Science 60, 7 (2014), 1792-1811.

[91] Shyam Boriah, Vipin Kumar, Michael Steinbach, Christopher Potter, and Steven Klooster. 2008. Land cover change detection: A case study. In 14th ACM SIGKDD International Conference on Knowledge Discovery and Data Mining. ACM, 857-865.

[92] Lior Bragilevsky and Ivan V. Bajić. 2017. Deep learning for Amazon satellite image analysis. In 2017 IEEE Pacific Rim Conference on Communications, Computers and Signal Processing (PACRIM'17). IEEE, 1-5.

[93] Simone Branchini, Francesco Pensa, Patrizia Neri, Bianca Maria Tonucci, Lisa Mattielli, Anna Collavo, Maria Elena Sillingardi, Corrado Piccinetti, Francesco Zaccanti, and Stefano Goffredo. 2015. Using a citizen science program to monitor coral reef biodiversity through space and time. Biodiversity and Conservation 24, 2 (2015), 319-336. 
[94] P. G. Brodrick, L. D. L. Anderegg, and G. P. Asner. 2019. Forest drought resistance at large geographic scales. Geophysical Research Letters 46, 5 (2019), 2752-2760.

[95] Austin Brown, Jeffrey Gonder, and Brittany Repac. 2014. An Analysis of Possible Energy Impacts of Automated Vehicles. Springer International Publishing, Cham, 137-153.

[96] T. Bruckner, I. A. Bashmakov, Y. Mulugetta, H. Chum, A. de la Vega Navarro, J. Edmonds, A. Faaij, B. Fungtammasan, A. Garg, E. Hertwich, D. Honnery, D. Infield, M. Kainuma, S. Khennas, S. Kim, H. B. Nimir, K. Riahi, N. Strachan, R. Wiser, and X. Zhang. 2014. Energy Systems, in IPCC, Working Group III Contribution to the Fifth Assessment Report of the Intergovernmental Panel on Climate Change, Climate Change 2014: Mitigation of Climate Change, chapter 8. Geneva. O. Edenhofer, R. Pichs-Madruga, Y. Sokona, E. Farahani, S. Kadner, K. Seyboth, A. Adler, I. Baum, S. Brunner, P. Eickemeier, B. Kriemann, J. Savolainen, S. Schlömer, C. von Stechow, T. Zwickel, and J. C. Minx (Eds.). Cambridge University Press, Cambridge.

[97] Emma Brunskill and Neal Lesh. 2010. Routing for rural health: Optimizing community health worker visit schedules. In 2010 AAAI Spring Symposium Series.

[98] Selma Brynolf, Maria Taljegard, Maria Grahn, and Julia Hansson. 2018. Electrofuels for the transport sector: A review of production costs. Renewable and Sustainable Energy Reviews 81, 2 (2018), 1887-1905.

[99] Niv Buchbinder, Navendu Jain, and Ishai Menache. 2011. Online job-migration for reducing the electricity bill in the cloud. In International Conference on Research in Networking. Springer, 172-185.

[100] R. V. Budny, R. Andre, G. Bateman, F. Halpern, C. E. Kessel, A. Kritz, and D. McCune. 2008. Predictions of H-mode Performance in ITER. Technical Report. Princeton Plasma Physics Lab, Princeton, NJ.

[101] Marshall Burke, Solomon M. Hsiang, and Edward Miguel. 2015. Global non-linear effect of temperature on economic production. Nature 527, 7577 (2015), 235.

[102] Fiona Burlig, Christopher Knittel, David Rapson, Mar Reguant, and Catherine Wolfram. 2017. Machine learning from schools about energy efficiency. Technical Report. National Bureau of Economic Research.

[103] Alex Burnap, Yanxin Pan, Ye Liu, Yi Ren, Honglak Lee, Richard Gonzalez, and Panos Y. Papalambros. 2016. Improving design preference prediction accuracy using feature learning. Journal of Mechanical Design 138, 7 (2016), 071404071404-12.

[104] Samuel Buteau and J. R. Dahn. 2019. Analysis of thousands of electrochemical impedance spectra of lithium-ion cells through a machine learning inverse model. fournal of The Electrochemical Society 166, 8 (2019), A1611-A1622.

[105] Keith T. Butler, Daniel W. Davies, Hugh Cartwright, Olexandr Isayev, and Aron Walsh. 2018. Machine learning for molecular and materials science. Nature 559, 7715 (2018), 547.

[106] Maya Cakmak and Andrea L. Thomaz. 2014. Eliciting good teaching from humans for machine learners. Artificial Intelligence 217 (2014), 198-215.

[107] Francesco Calivá, Fabio Sousa De Ribeiro, Antonios Mylonakis, Christophe Demazière, Paolo Vinai, Georgios Leontidis, and Stefanos Kollias. 2018. A deep learning approach to anomaly detection in nuclear reactors. In 2018 International foint Conference on Neural Networks (IfCNN'18). IEEE, 1-8.

[108] Emanuele Campiglio, Yannis Dafermos, Pierre Monnin, Josh Ryan-Collins, Guido Schotten, and Misa Tanaka. 2018. Climate change challenges for central banks and financial regulators. Nature Climate Change 8, 6 (2018), 462.

[109] Camus Energy. 2019. Camus Energy. Retrieved from https://camus.energy/.

[110] Barbara Cannas, Alessandra Fanni, E. Marongiu, and P. Sonato. 2003. Disruption forecasting at JET using neural networks. Nuclear fusion 44, 1 (2003), 68.

[111] Zachary P. Cano, Dustin Banham, Siyu Ye, Andreas Hintennach, Jun Lu, Michael Fowler, and Zhongwei Chen. 2018. Batteries and fuel cells for emerging electric vehicle markets. Nature Energy 3, 4 (2018), 279-289.

[112] Carbon Engineering. 2021. Carbon Engineering. Retrieved from https://carbonengineering.com/.

[113] Carbon Mapper. 2021. Carbon Mapper. Retrieved from https://carbonmapper.org/.

[114] Carbon Tracker. 2019. Carbon Tracker to Measure World's Power Plant Emissions from Space with Support from Google.org. Retrieved from https://www.carbontracker.org/carbon-tracker-to-measure-worlds-power-plantemissions-from-space-with-support-from-google-org/.

[115] J. Carman, T. Clune, F. Giraldo, M. Govett, B. Gross, A. Kamrathe, T. Lee, D. McCarren, J. Michalakes, S. Sandgathe, and T. Whitcomb. 2017. Position paper on high performance computing needs in Earth system prediction. National Earth System Prediction Capability. Technical Report. Retrived from https://doi.org/10.7289/V5862DH3

[116] Simone Carr-Cornish, Peta Ashworth, John Gardner, and Stephen J. Fraser. 2011. Exploring the orientations which characterise the likely public acceptance of low emission energy technologies. Climatic Change 107, 3-4 (2011), 549565.

[117] Veronica B. Cashin, Daniel S. Eldridge, Aimin Yu, and Dongyuan Zhao. 2018. Surface functionalization and manipulation of mesoporous silica adsorbents for improved removal of pollutants: A review. Environmental Science: Water Research \& Technology 4, 2 (2018), 110-128. 
[118] Carlos Castillo. 2016. Big Crisis Data: Social Media in Disasters and Time-Critical Situations. Cambridge University Press.

[119] M. A. Celia, S. Bachu, J. M. Nordbotten, and K. W. Bandilla. 2015. Status of CO2 storage in deep saline aquifers with emphasis on modeling approaches and practical simulations. Water Resources Research 51, 9 (2015), 6846-6892.

[120] Martin Cenek, Rocco Haro, Brandon Sayers, and Jifeng Peng. 2018. Climate Change and Power Security: Power Load Prediction for Rural Electrical Microgrids Using Long Short Term Memory and Artificial Neural Networks. Applied Sciences 8, 5 (2018), 749.

[121] Greifswald Mire Centre. 2021. Global Peatland Database. Retrieved from https://greifswaldmoor.de/global-peatlanddatabase-en.html.

[122] A. Chaabane, A. Ramudhin, and M. Paquet. 2012. Design of sustainable supply chains under the emission trading scheme. International fournal of Production Economics 135, 1 (2012), 37-49. Advances in Optimization and Design of Supply Chains.

[123] S. Chakraborty and A. C. Newton. 2011. Climate change, plant diseases and food security: An overview. Plant Pathology 60, 1 (2011), 2-14.

[124] Devendra Singh Chaplot, Christopher MacLellan, Ruslan Salakhutdinov, and Kenneth Koedinger. 2018. Learning cognitive models using neural networks. In International Conference on Artificial Intelligence in Education. Springer, 43-56.

[125] Bailian Chen, Dylan R. Harp, Youzuo Lin, Elizabeth H. Keating, and Rajesh J. Pawar. 2018. Geologic CO2 sequestration monitoring design: A machine learning and uncertainty quantification based approach. Applied Energy 225 (2018), $332-345$.

[126] Chi-Hua Chen, Hsu-Yang Kung, and Feng-Jang Hwang. 2019. Deep learning techniques for agronomy applications. Agronomy 9, 3 (2019), 142.

[127] Ching-Ho Chen, Wei-Lin Liu, and Chia-Hsing Chen. 2006. Development of a multiple objective planning theory and system for sustainable air quality monitoring networks. Science of The Total Environment 354, 1 (2006), 1-19.

[128] Di Chen and Carla P. Gomes. 2019. Bias reduction via end-to-end shift learning: Application to citizen science. In 33rd AAAI Conference on Artificial Intelligence.

[129] Fu-Chen Chen and Mohammad R. Jahanshahi. 2018. NB-CNN: Deep learning-based crack detection using convolutional neural network and naïve Bayes data fusion. IEEE Transactions on Industrial Electronics 65, 5 (2018), 4392-4400.

[130] Jie Chen, Kees de Hoogh, Maciek Strak, Jules Kerckhoffs, Roel Vermeulen, Bert Brunekreef, and Gerard Hoek. 2018. OP III-4 Exposure assessment models for NO2 and PM2.5 in the elapse study: A comparison of supervised linear regression and machine learning approaches. Occupational and Environmental Medicine 75, Suppl 1 (2018), A6.

[131] T. Donna Chen, Kara M. Kockelman, and Josiah P. Hanna. 2016. Operations of a shared, autonomous, electric vehicle fleet: Implications of vehicle \& charging infrastructure decisions. Transportation Research Part A: Policy and Practice 94 (2016), 243-254.

[132] Tian Qi Chen, Yulia Rubanova, Jesse Bettencourt, and David K. Duvenaud. 2018. Neural ordinary differential equations. In Advances in Neural Information Processing Systems. 6571-6583.

[133] Xiqun (Michael) Chen, Majid Zahiri, and Shuaichao Zhang. 2017. Understanding ridesplitting behavior of on-demand ride services: An ensemble learning approach. Transportation Research Part C: Emerging Technologies 76 (2017), 51-70.

[134] City of Los Angeles. 2018. Mobility Data Specification. Retrieved from https://github.com/CityOfLosAngeles/ mobility-data-specification.git.

[135] Benjamin Clement, Didier Roy, Pierre-Yves Oudeyer, and Manuel Lopes. 2013. Multi-armed bandits for intelligent tutoring systems. fournal of Educational Data Mining 7, 2 (2013), 20-48.

[136] Climeworks. 2021. Climeworks. Retrieved from https://www.climeworks.com/.

[137] Nicholas Clinton and Peng Gong. 2013. MODIS detected surface urban heat islands and sinks: Global locations and controls. Remote Sensing of Environment 134 (2013), 294-304.

[138] Brendan Coffey. 2019. Factory Records: GE Providing Procter \& Gamble Greater Access To The Cloud For Analyzing Manufacturing Data. Retrived from https://www.ge.com/reports/factory-records-ge-providing-procter-gamblegreater-access-cloud-analyzing-manufacturing-data/.

[139] Judah Cohen, Dim Coumou, Jessica Hwang, Lester Mackey, Paulo Orenstein, Sonja Totz, and Eli Tziperman. 2018. S2S reboot: An argument for greater inclusion of machine learning in subseasonal to seasonal forecasts. WIREs Climate Change 10, 2 (2018), e00567.

[140] Connor W. Coley, Wengong Jin, Luke Rogers, Timothy F. Jamison, Tommi S. Jaakkola, William H. Green, Regina Barzilay, and Klavs F. Jensen. 2019. A graph-convolutional neural network model for the prediction of chemical reactivity. Chemical Science 10, 2 (2019), 370-377.

[141] Federal Energy Regulatory Commission. 2015. Energy Primer: A Handbook of Energy Market Basics. Federal Energy Regulatory Commission, Washington, DC. 
[142] Eugene C. Cordero, Anne Marie Todd, and Diana Abellera. 2008. Climate change education and the ecological footprint. Bulletin of the American Meteorological Society 89, 6 (2008), 865-872.

[143] Olivier Corradi. 2018. Estimating the marginal carbon intensity of electricity with machine learning. Retrieved from https://medium.com/electricitymap/using-machine-learning-to-estimate-the-hourly-marginal-carbon-intensityof-electricity-49eade43b421.

[144] Nick Couldry and Ulises A. Mejias. 2019. Data colonialism: Rethinking big data's relation to the contemporary subject. Television \& New Media 20, 4 (2019), 336-349.

[145] Natural Resources Defense Council. 2015. Geoengineering: Research is Prudent, But No Substitute for Carbon Pollution Cuts. Retrieved from https://www.nrdc.org/media/2015/150210.

[146] Steven C. Cowley. 2016. The quest for fusion power. Nature Physics 12, 5 (2016), 384.

[147] Auslan Cramb. 2006. 12,000-mile trip to have seafood shelled. The Telegraph.

[148] A. Crane-Droesch, B. Kravitz, and J. T. Abatzoglou. 2018. Using deep learning to model potential impacts of geoengineering via solar radiation management on US agriculture. In AGU Fall Meeting Abstracts.

[149] Felix Creutzig. 2016. Economic and ecological views on climate change mitigation with bioenergy and negative emissions. GCB Bioenergy 8, 1 (2016), 4-10.

[150] Felix Creutzig, Peter Agoston, Jan C. Minx, Josep G. Canadell, Robbie M. Andrew, Corinne Le Quéré, Glen P. Peters, Ayyoob Sharifi, Yoshiki Yamagata, and Shobhakar Dhakal. 2016. Urban infrastructure choices structure climate solutions. Nature Climate Change 6, 12 (2016), 1054-1056.

[151] Felix Creutzig, Giovanni Baiocchi, Robert Bierkandt, Peter-Paul Pichler, and Karen C. Seto. 2015. Global typology of urban energy use and potentials for an urbanization mitigation wedge. Proceedings of the National Academy of Sciences 112, 20 (2015), 6283-6288.

[152] Felix Creutzig, Christian Breyer, Jerome Hilaire, Jan Minx, Glen Peters, and Robert H. Socolow. 2019. The mutual dependence of negative emission technologies and energy systems. Energy \& Environmental Science 12, 6 (2019), 1805-1817.

[153] Felix Creutzig, Martina Franzen, Rolf Moeckel, Dirk Heinrichs, Kai Nagel, and Helga Weisz. 2019. Leveraging digitalization for sustainability in urban transport. Global Sustainability 2 (2019), E14.

[154] Felix Creutzig, Patrick Jochem, Oreane Y. Edelenbosch, Linus Mattauch, Detlef P. van Vuuren, David McCollum, and Jan Minx. 2015. Transport: A roadblock to climate change mitigation? Science 350, 6263 (2015), 911-912.

[155] Felix Creutzig, N. H. Ravindranath, Göran Berndes, Simon Bolwig, Ryan Bright, Francesco Cherubini, Helena Chum, Esteve Corbera, Mark Delucchi, Andre Faaij, Joseph Fargione, Helmut Haberl, Garvin Heath, Oswaldo Lucon, Richard Plevin, Alexander Popp, Carmenza Robledo-Abad, Steven Rose, Pete Smith, Anders Stromman, Sangwon Suh, and Omar Masera. 2015. Bioenergy and climate change mitigation: An assessment. GCB Bioenergy 7, 5 (2015), 916-944.

[156] Sina Dabiri and Kevin Heaslip. 2018. Inferring transportation modes from GPS trajectories using a convolutional neural network. Transportation Research Part C: Emerging Technologies 86 (2018), 360-371.

[157] Aiguo Dai. 2011. Drought under global warming: A review. Wiley Interdisciplinary Reviews: Climate Change 2, 1 (2011), 45-65.

[158] Xiaoqing Dai, Lijun Sun, and Yanyan Xu. 2018. Short-term origin-destination based metro flow prediction with probabilistic model selection approach. fournal of Advanced Transportation 2018, 2399 (2018), 1-15.

[159] Hari Prasanna Das, Ioannis C. Konstantakopoulos, Aummul Baneen Manasawala, Tanya Veeravalli, Huihan Liu, and Costas J. Spanos. 2019. A novel graphical lasso based approach towards segmentation analysis in energy gametheoretic frameworks. In 2019 18th IEEE International Conference On Machine Learning And Applications (ICMLA'19). IEEE, 1702-1709.

[160] Jnaneshwar Das, Frédéric Py, Julio B. J. Harvey, John P. Ryan, Alyssa Gellene, Rishi Graham, David A. Caron, Kanna Rajan, and Gaurav S. Sukhatme. 2015. Data-driven robotic sampling for marine ecosystem monitoring. The International fournal of Robotics Research 34, 12 (2015), 1435-1452.

[161] Utpal Kumar Das, Kok Soon Tey, Mehdi Seyedmahmoudian, Saad Mekhilef, Moh Yamani Idna Idris, Willem Van Deventer, Bend Horan, and Alex Stojcevski. 2018. Forecasting of photovoltaic power generation and model optimization: A review. Renewable and Sustainable Energy Reviews 81, 1 (2018), 912-928.

[162] Steven J. Davis, Nathan S. Lewis, Matthew Shaner, Sonia Aggarwal, Doug Arent, Inês L. Azevedo, Sally M. Benson, Thomas Bradley, Jack Brouwer, Yet-Ming Chiang, Christopher T. M. Clack, Armond Cohen, Stephen Doig, Jae Edmonds, Paul Fennell, Christopher B. Field, Bryan Hannegan, Bri-Mathias Hodge, Martin I. Hoffert, Eric Ingersoll, Paulina Jaramillo, Klaus S. Lackner, Katharine J. Mach, Michael Mastrandrea, Joan Ogden, Per F. Peterson, Daniel L. Sanchez, Daniel Sperling, Joseph Stagner, Jessika E. Trancik, Chi-Jen Yang, and Ken Caldeira. 2018. Net-zero emissions energy systems. Science 360, 6396 (2018).

[163] Maria De-Arteaga, William Herlands, Daniel B. Neill, and Artur Dubrawski. 2018. Machine learning for the developing world. ACM Transactions on Management Information Systems 9, 2 (2018), 9. 
[164] Filipe de Avila Belbute-Peres, Kevin Smith, Kelsey Allen, Josh Tenenbaum, and J. Zico Kolter. 2018. End-to-end differentiable physics for learning and control. In Advances in Neural Information Processing Systems. 7178-7189.

[165] Julian de Hoog, Stefan Maetschke, Peter Ilfrich, and Ramachandra Rao Kolluri. 2020. Using satellite and aerial imagery for identification of solar PV: State of the art and research opportunities. In 11th ACM International Conference on Future Energy Systems. 308-313.

[166] Cristóbal De La Maza, Alex Davis, Cleotilde Gonzalez, and Inês Azevedo. 2018. A graph-based model to discover preference structure from choice data. In 40th Annual Meeting of the Cognitive Science Society (CogSci'18). 25-28.

[167] Cristóbal de la Maza Guzmán. 2013. Willingness to pay to avoid environmental impacts of electricity generation. Technical Report. Latin American and Caribbean Environmental Economics Program.

[168] Scott De Marchi and Scott E. Page. 2014. Agent-based models. Annual Review of Political Science 17 (2014), 1-20.

[169] Juan F. De Paz, Javier Bajo, Sara Rodríguez, Gabriel Villarrubia, and Juan M. Corchado. 2016. Intelligent system for lighting control in smart cities. Information Sciences 372 (2016), 241-255.

[170] Christian Schroeder de Witt and Thomas Hornigold. 2019. Stratospheric aerosol injection as a deep reinforcement learning problem. In ICML 2019 Workshop on Climate Change: How Can AI Help?

[171] Adeline Decuyper, Alex Rutherford, Amit Wadhwa, Jean-Martin Bauer, Gautier Krings, Thoralf Gutierrez, Vincent D. Blondel, and Miguel A. Luengo-Oroz. 2014. Estimating food consumption and poverty indices with mobile phone data. Preprint arXiv:1412.2595 (2014).

[172] Chris Dede. 2009. Immersive interfaces for engagement and learning. Science 323, 5910 (2009), 66-69.

[173] Matthias Deindl, Carsten Block, Rustam Vahidov, and Dirk Neumann. 2008. Load shifting agents for automated demand side management in micro energy grids. In 2008 2nd IEEE International Conference on Self-Adaptive and Self-Organizing Systems. IEEE, 487-488.

[174] I. Delpla, A.-V. Jung, E. Baures, M. Clement, and O. Thomas. 2009. Impacts of climate change on surface water quality in relation to drinking water production. Environment International 35, 8 (2009), 1225-1233.

[175] Mark A. Delucchi, James J. Murphy, and Donald R. McCubbin. 2002. The health and visibility cost of air pollution: A comparison of estimation methods. Fournal of Environmental Management 64, 2 (2002), 139-152.

[176] Dendra Systems. 2021. Dendra Systems. Retrieved from https://dendra.io/.

[177] Zhipeng Deng, Hao Sun, Shilin Zhou, Juanping Zhao, and Huanxin Zou. 2017. Toward fast and accurate vehicle detection in aerial images using coupled region-based convolutional neural networks. IEEE fournal of Selected Topics in Applied Earth Observations and Remote Sensing 10, 8 (2017), 3652-3664.

[178] Ethan W. Dereszynski and Thomas G. Dietterich. 2007. Probabilistic models for anomaly detection in remote sensor data streams. In Proceedings of the 23rd conference on uncertainty in artificial intelligence (UAI-2007), Vancouver, BC. Corvallis, OR. AUAI Press, 75-82.

[179] Qian Di, Itai Kloog, Petros Koutrakis, Alexei Lyapustin, Yujie Wang, and Joel Schwartz. 2016. Assessing PM2.5 exposures with high spatiotemporal resolution across the continental United States. Environmental Science \& Technology 50, 9 (2016), 4712-4721.

[180] Qian Di, Petros Koutrakis, Christine Choirat, Francesca Dominici, and Joel D. Schwartz. 2018. Machine learning approach for spatially and temporally resolved PM2.5 exposures in the continental United States. In ISEE Conference Abstracts.

[181] Riccardo Di Clemente, Miguel Luengo-Oroz, Matias Travizano, Sharon Xu, Bapu Vaitla, and Marta C. González. 2018. Sequences of purchases in credit card data reveal lifestyles in urban populations. Nature Communications 9 (2018), 3330.

[182] Ivan Diaz-Rainey, Becky Robertson, and Charlie Wilson. 2017. Stranded research? Leading finance journals are silent on climate change. Climatic Change 143, 1-2 (2017), 243-260.

[183] Jan Philipp Dietrich, Alexander Popp, and Hermann Lotze-Campen. 2013. Reducing the loss of information and gaining accuracy with clustering methods in a global land-use model. Ecological Modelling 263 (2013), 233-243.

[184] Thomas G. Dietterich. 2009. Machine learning in ecosystem informatics and sustainability. In 21st International foint Conference on Artificial Intelligence.

[185] Simon Dietz, Alex Bowen, Charlie Dixon, and Philip Gradwell. 2016. 'Climate value at risk' of global financial assets. Nature Climate Change 6, 7 (2016), 676.

[186] Djellel Eddine Difallah, Philippe Cudre-Mauroux, and Sean A. McKenna. 2013. Scalable anomaly detection for smart city infrastructure networks. IEEE Internet Computing 17, 6 (2013), 39-47.

[187] Noah S. Diffenbaugh and Marshall Burke. 2019. Global warming has increased global economic inequality. Proceedings of the National Academy of Sciences 116, 20 (2019), 9808-9813.

[188] Bistra Dilkina, Jayant R. Kalagnanam, and Elena Novakovskaia. 2015. Method for designing the layout of turbines in a windfarm. US Patent No. 9,189,570.

[189] Chuan Ding, Xinyu Jason Cao, and Petter Næss. 2018. Applying gradient boosting decision trees to examine nonlinear effects of the built environment on driving distance in Oslo. Transportation Research Part A: Policy and Practice 110 (2018), 107-117.

ACM Computing Surveys, Vol. 55, No. 2, Article 42. Publication date: February 2022. 
[190] AnHai Doan, Jayant Madhavan, Pedro Domingos, and Alon Halevy. 2004. Ontology matching: A machine learning approach. In Handbook on Ontologies. Springer, 385-403.

[191] Roel Dobbe, David Fridovich-Keil, and Claire Tomlin. 2017. Fully decentralized policies for multi-agent systems: An information theoretic approach. In Advances in Neural Information Processing Systems. 2941-2950.

[192] Roel Dobbe, Oscar Sondermeijer, David Fridovich-Keil, Daniel Arnold, Duncan Callaway, and Claire Tomlin. 2019. Towards distributed energy services: Decentralizing optimal power flow with machine learning. IEEE Transactions on Smart Grid 11, 2 (2019), 1296-1306.

[193] Simona D’Oca and Tianzhen Hong. 2015. Occupancy schedules learning process through a data mining framework. Energy and Buildings 88 (2015), 395-408.

[194] Francesca Dominici, Roger D. Peng, Michelle L. Bell, Luu Pham, Aidan McDermott, Scott L. Zeger, and Jonathan M. Samet. 2006. Fine particulate air pollution and hospital admission for cardiovascular and respiratory diseases. FAMA 295, 10 (2006), 1127-1134.

[195] Bing Dong, Zhaoxuan Li, S. M. Mahbobur Rahman, and Rolando Vega. 2016. A hybrid model approach for forecasting future residential electricity consumption. Energy and Buildings 117 (2016), 341-351.

[196] Wenqian Dong, Zhen Xie, Gokcen Kestor, and Dong Li. 2020. Smart-PGSim: Using neural network to accelerate ACOPF power grid simulation. In SC20: International Conference for High Performance Computing, Networking, Storage and Analysis. IEEE, 1-15.

[197] Benjamin Donnot, Isabelle Guyon, Marc Schoenauer, Patrick Panciatici, and Antoine Marot. 2017. Introducing machine learning for power system operation support. Preprint arXiv:1709.09527 (2017).

[198] Priya Donti, Brandon Amos, and J. Zico Kolter. 2017. Task-based end-to-end model learning in stochastic optimization. In Advances in Neural Information Processing Systems. 5484-5494.

[199] Priya L. Donti, Liu Yajing, Andreas J. Schmitt, Andrey Bernstein, Rui Yang, and Yingchen Zhang. 2019. Matrix completion for low-observability voltage estimation. IEEE Transactions on Smart Grid 11, 3 (2019), 2520-2530.

[200] Jigar Doshi, Saikat Basu, and Guan Pang. 2018. From satellite imagery to disaster insights. Preprint arXiv:1812.07033 (2018).

[201] Otilia Elena Dragomir, Rafael Gouriveau, Florin Dragomir, Eugenia Minca, and Noureddine Zerhouni. 2009. Review of prognostic problem in condition-based maintenance. In 2009 European Control Conference (ECC'09). IEEE, 1587-1592.

[202] Ján Drgoňa, Damien Picard, Michal Kvasnica, and Lieve Helsen. 2018. Approximate model predictive building control via machine learning. Applied Energy 218 (2018), 199-216.

[203] DrivenData. 2019. Mapping Agricultural Supply Chains from Source to Shelf. Retrieved from http://drivendata.co/ case-studies/mapping-agricultural-supply-chains-from-source-to-shelf/.

[204] DroneSeed. 2021. DroneSeed. Retrieved from https://droneseed.com/.

[205] Xinya Du, Junru Shao, and Claire Cardie. 2017. Learning to ask: Neural question generation for reading comprehension. In 55th Annual Meeting of the Association for Computational Linguistics.

[206] Victor Duarte. 2018. Machine Learning for Continuous-Time Economics. (2018). Retrieved from https://doi.org/10. 2139/ssrn.3012602

[207] Dominic Dudley. 2018. Renewable Energy Will Be Consistently Cheaper Than Fossil Fuels By 2020, Report Claims. Retrieved from https://www.forbes.com/sites/dominicdudley/2018/01/13/renewable-energy-cost-effectivefossil-fuels-2020/.

[208] Matthew Dunbabin and Lino Marques. 2012. Robots for environmental monitoring: Significant advancements and applications. IEEE Robotics \& Automation Magazine 19, 1 (2012), 24-39.

[209] J. A. Dykema, D. W. Keith, and F. N. Keutsch. 2016. Improved aerosol radiative properties as a foundation for solar geoengineering risk assessment. Geophysical Research Letters 43, 14 (2016), 7758-7766.

[210] Sebastian D. Eastham, Debra K. Weisenstein, David W. Keith, and Steven R. H. Barrett. 2018. Quantifying the impact of sulfate geoengineering on mortality from air quality and UV-B exposure. Atmospheric Environment 187 (2018), 424-434.

[211] Imme Ebert-Uphoff, David Thompson, Ibrahim Demir, Yulia Gel, Mary Hill, Anuj Karpatne, Mariana Guereque, Vipin Kumar, Enrique Cabal-Cano, and Padhraic Smyth. 2017. A vision for the development of benchmarks to bridge geoscience and data science. In 17th International Workshop on Climate Informatics.

[212] ecoRobotix. 2021. ecoRobotix. Retrieved from https://www.ecorobotix.com/en/.

[213] Tim Edward and Rob Salkowitz. 2018. How machine learning contributes to smarter pipeline maintenance. Retrieved from https://www.oilandgaseng.com/articles/how-machine-learning-contributes-to-smarter-pipelinemaintenance/.

[214] P. N. Edwards. 2010. History of climate modeling. Wiley Interdisciplinary Reviews: Climate Change 2, 1 (2010), $128-139$. Issue 1. 
[215] Karen Ehrhardt-Martinez, Kat A. Donnelly, and John A. Skip Laitner. 2010. Advanced metering initiatives and residential feedback programs: A meta-review for household electricity-saving opportunities. American Council for an Energy-Efficient Economy, Washington, DC.

[216] Carl Elkin and Sims Witherspoon. 2019. Machine learning can boost the value of wind energy. Retrieved from https: //deepmind.com/blog/machine-learning-can-boost-value-wind-energy/.

[217] A. Denny Ellerman, Frank J. Convery, and Christian De Perthuis. 2010. Pricing Carbon: The European Union Emissions Trading Scheme. Cambridge University Press.

[218] L. D. Ellis, S. Buteau, Samuel G. Hames, L. M. Thompson, D. S. Hall, and J. R. Dahn. 2018. A new method for determining the concentration of electrolyte components in lithium-ion cells, using fourier transform infrared spectroscopy and machine learning. Journal of The Electrochemical Society 165, 2 (2018), A256-A262.

[219] Douglas Douglas Austin Ellman. 2015. The reference electrification model: A computer model for planning rural electricity access. Ph.D. Dissertation. Massachusetts Institute of Technology.

[220] Adam N. Elmachtoub and Paul Grigas. 2021. Smart "Predict, then Optimize". Management Science (2021).

[221] Robert F. Engle, Stefano Giglio, Heebum Lee, Bryan T. Kelly, and Johannes Stroebel. 2020. Hedging climate change news. The Review of Financial Studies 33, 3 (2019), 1184-1216.

[222] Joshua M. Epstein. 2006. Generative Social Science: Studies in Agent-Based Computational Modeling. Princeton University Press.

[223] Alireza Ermagun and David Levinson. 2018. Spatiotemporal traffic forecasting: Review and proposed directions. Transport Reviews 38, 6 (2018), 786-814.

[224] Thomas Esch, Wieke Heldens, Andreas Hirner, Manfred Keil, Mattia Marconcini, Achim Roth, Julian Zeidler, Stefan Dech, and Emanuele Strano. 2017. Breaking new ground in mapping human settlements from space-The global urban footprint. ISPRS Journal of Photogrammetry and Remote Sensing 134 (2017), 30-42.

[225] Andreas Essl, André Ortner, Reinhard Haas, and Peter Hettegger. 2017. Machine learning analysis for a flexibility energy approach towards renewable energy integration with dynamic forecasting of electricity balancing power. In 2017 14th International Conference on the European Energy Market (EEM’17). IEEE, 1-6.

[226] Annette Evans, Vladimir Strezov, and Tim J. Evans. 2012. Assessment of utility energy storage options for increased renewable energy penetration. Renewable and Sustainable Energy Reviews 16, 6 (2012), 4141-4147.

[227] Richard Evans and Jim Gao. 2016. DeepMind AI reduces Google data centre cooling bill by $40 \%$. Retrieved from https://deepmind.com/blog/article/deepmind-ai-reduces-google-data-centre-cooling-bill-40.

[228] Reid Ewing and Robert Cervero. 2017. "Does Compact Development Make People Drive Less?” The Answer Is Yes. fournal of the American Planning Association 83, 1 (2017), 19-25.

[229] Luc Eyraud, Benedict Clements, and Abdoul Wane. 2013. Green investment: Trends and determinants. Energy Policy 60 (2013), 852-865.

[230] V. Eyring, S. Bony, G. A. Meehl, C. A. Senior, R. J. Stouffer, and K. E. Taylor. 2016. Overview of the coupled model intercomparison project phase 6 (CMIP6) experimental design and organization. Geoscientific Model Development 9 , 5 (2016), 1937-1958

[231] James H. Faghmous and Vipin Kumar. 2014. A big data guide to understanding climate change: The case for theoryguided data science. Big Data 2, 3 (2014), 155-163.

[232] Robin Faillettaz, Marc Picheral, Jessica Y. Luo, Cédric Guigand, Robert K. Cowen, and Jean-Olivier Irisson. 2016. Imperfect automatic image classification successfully describes plankton distribution patterns. Methods in Oceanography 15 (2016), 60-77.

[233] Guochang Fang, Lixin Tian, Min Fu, Mei Sun, Ruijin Du, and Menghe Liu. 2017. Investigating carbon tax pilot in YRD urban agglomerations-Analysis of a novel ESER system with carbon tax constraints and its application. Applied Energy 194 (2017), 635-647.

[234] Guochang Fang, Lixin Tian, Menghe Liu, Min Fu, and Mei Sun. 2018. How to optimize the development of carbon trading in China-Enlightenment from evolution rules of the EU carbon price. Applied Energy 211 (2018), 1039-1049.

[235] Xi Fang, Satyajayant Misra, Guoliang Xue, and Dejun Yang. 2012. Smart grid-The new and improved power grid: A survey. IEEE Communications Surveys \& Tutorials 14, 4 (2012), 944-980.

[236] F. Felici and O. Sauter. 2012. Non-linear model-based optimization of actuator trajectories for tokamak plasma profile control. Plasma Physics and Controlled Fusion 54, 2 (2012), 025002.

[237] F. Felici, O. Sauter, S. Coda, B. P. Duval, T. P. Goodman, J. M. Moret, J. I. Paley, and TCV Team. 2011. Real-time physicsmodel-based simulation of the current density profile in tokamak plasmas. Nuclear Fusion 51, 8 (2011), 083052.

[238] Xiaohui Feng, María Uriarte, Grizelle González, Sasha Reed, Jill Thompson, Jess K. Zimmerman, and Lora Murphy. 2018. Improving predictions of tropical forest response to climate change through integration of field studies and ecosystem modeling. Global Change Biology 24, 1 (2018), e213-e232.

[239] Christopher B. Field, Vicente Barros, Thomas F. Stocker, and Qin Dahe. 2012. Managing the Risks of Extreme Events and Disasters to Advance Climate Change Adaptation: Special Report of the Intergovernmental Panel on Climate Change. Cambridge University Press. 
[240] Maria Figueroa, Oliver Lah, Lewis M. Fulton, Alan McKinnon, and Geetam Tiwari. 2014. Energy for transport. Annual Review of Environment and Resources 39 (2014), 295-325.

[241] Matt Finer, Sidney Novoa, Mikaela J. Weisse, Rachael Petersen, Joseph Mascaro, Tamia Souto, Forest Stearns, and Raúl García Martinez. 2018. Combating deforestation: From satellite to intervention. Science 360, 6395 (2018), 13031305.

[242] Ferdinando Fioretto, Terrence W. K. Mak, and Pascal Van Hentenryck. 2020. Predicting AC optimal power flows: Combining deep learning and lagrangian dual methods. In AAAI Conference on Artificial Intelligence. Vol. 34. 630637.

[243] Manfred Fischedick, Joyashree Roy, Amr Abdel-Aziz, Adolf Acquaye, Julian Allwood, Jean-Paul Ceron, Yong Geng, Haroon Kheshgi, Alessandro Lanza, Daniel Perczyk, Lynn Price, Estela Santalla, Claudia Sheinbaum, and Kanako Tanaka. 2014. Industry. In Climate Change 2014: Mitigation of Climate Change. Contribution of Working Group III to the Fifth Assessment Report of the Intergovernmental Panel on Climate Change, O. Edenhofer, R. Pichs-Madruga, Y Sokona, E. Farahani, S. Kadner, K. Seyboth, A. Adler, I. Baum, S. Brunner, P. Eickemeier, B. Kriemann, J. Savolainen, S. Schlömer, C. von Stechow, T. Zwickel, and J. C. Minx (Eds.). Cambridge University Press.

[244] Douglas H. Fisher, Zimei Bian, and Selina Chen. 2016. Incorporating sustainability into computing education. IEEE Intelligent Systems 31, 5 (2016), 93-96.

[245] Mike Flannigan, Chelene Krezek-Hanes, Mike Wotton, Mike Waddington, Merritt Turetsky, and Brian Benscoter. 2012. Peatland Fires and Carbon Emissions (Bulletin 50). Technical Report.

[246] Genevieve Flaspohler, Nicholas Roy, and Yogesh Girdhar. 2017. Feature discovery and visualization of robot mission data using convolutional autoencoders and Bayesian nonparametric topic models. In 2017 IEEE/RSf International Conference on Intelligent Robots and Systems (IROS'17). IEEE, 1-8.

[247] Christopher G. Fletcher, Ben Kravitz, and Bakr Badawy. 2018. Quantifying uncertainty from aerosol and atmospheric parameters and their impact on climate sensitivity. Atmospheric Chemistry and Physics 18, 23 (2018), 17529-17543.

[248] Sarah Fletcher, Megan Lickley, and Kenneth Strzepek. 2019. Learning about climate change uncertainty enables flexible water infrastructure planning. Nature Communications 10, 1 (2019), 1782.

[249] Christian Folberth, Artem Baklanov, Juraj Balkovič, Rastislav Skalskỳ, Nikolay Khabarov, and Michael Obersteiner. 2019. Spatio-temporal downscaling of gridded crop model yield estimates based on machine learning. Agricultural and Forest Meteorology 264 (2019), 1-15.

[250] Samantha Foley. 2011. Integrated Plasma Simulator (IPS) v2.1 documentation. Retrieved from http://ipsframework. sourceforge.net/doc/html/.

[251] James D. Ford, Simon E. Tilleard, Lea Berrang-Ford, Malcolm Araos, Robbert Biesbroek, Alexandra C. Lesnikowski, Graham K. MacDonald, Angel Hsu, Chen Chen, and Livia Bizikova. 2016. Opinion: Big data has big potential for applications to climate change adaptation. Proceedings of the National Academy of Sciences 113, 39 (2016), 1072910732.

[252] Vanessa Frias-Martinez, Cristina Soguero, and Enrique Frias-Martinez. 2012. Estimation of urban commuting patterns using cellphone network data. In ACM SIGKDD International Workshop on Urban Computing. ACM, 9-16.

[253] Vanessa Frias-Martinez, Victor Soto, Jesus Virseda, and Enrique Frias-Martinez. 2012. Computing cost-effective census maps from cell phone traces. In Workshop on Pervasive Urban Applications.

[254] David Friederich, Lynn H. Kaack, Alexandra Luccioni, and Bjarne Steffen. 2021. Automated identification of climate risk disclosures in annual corporate reports. arXiv preprint arXiv:2108.01415 (2021).

[255] Guoyin Fu. 2018. Deep belief network based ensemble approach for cooling load forecasting of air-conditioning system. Energy 148 (2018), 269-282.

[256] Guillermo Fuertes, Ismael Soto, Raúl Carrasco, Manuel Vargas, Jorge Sabattin, and Carolina Lagos. 2016. Intelligent packaging systems: Sensors and nanosensors to monitor food quality and safety. fournal of Sensors 2016, 2 (2016), 1-8.

[257] Koji Fujimura, Atsuto Seko, Yukinori Koyama, Akihide Kuwabara, Ippei Kishida, Kazuki Shitara, Craig A. J. Fisher, Hiroki Moriwake, and Isao Tanaka. 2013. Accelerated materials design of lithium superionic conductors based on first-principles calculations and machine learning algorithms. Advanced Energy Materials 3, 8 (2013), 980-985.

[258] Environmental Defense Fund. 2019. Our position on geoengineering. Retrieved from https://www.edf.org/climate/ our-position-geoengineering.

[259] Sabine Fuss, Josep G. Canadell, Glen P. Peters, Massimo Tavoni, Robbie M. Andrew, Philippe Ciais, Robert B. Jackson, Chris D. Jones, Florian Kraxner, Nebosja Nakicenovic, Corinne Le Quéré, Michael R. Raupach, Ayyoob Sharifi, Pete Smith, and Yoshiki Yamagata. 2014. Betting on negative emissions. Nature Climate Change 4, 10 (2014), 850.

[260] Sabine Fuss, William F. Lamb, Max W. Callaghan, Jérôme Hilaire, Felix Creutzig, Thorben Amann, Tim Beringer, Wagner de Oliveira Garcia, Jens Hartmann, Tarun Khanna, Gunnar Luderer, Gregory F. Nemet, Joeri Rogelj, Pete Smith, José Luis Vicente Vicente, Jennifer Wilcox, Maria del Mar Zamora Dominguez, and Jan C. Minx. 2018. Negative emissions-Part 2: Costs, potentials and side effects. Environmental Research Letters 13, 6 (2018), 063002. 
[261] Elizabeth Gabe-Thomas, Ian Walker, Bas Verplanken, and Gavin Shaddick. 2016. Householders' mental models of domestic energy consumption: Using a sort-and-cluster method to identify shared concepts of appliance similarity. PloS One 11, 7 (2016), e0158949.

[262] David John Gagne, Amy McGovern, Sue Ellen Haupt, Ryan A. Sobash, John K. Williams, and Ming Xue. 2017. Stormbased probabilistic hail forecasting with machine learning applied to convection-allowing ensembles. Weather and Forecasting 32, 5 (2017), 1819-1840.

[263] M.-È. Gagné, N. P. Gillett, and J. C. Fyfe. 2015. Observed and simulated changes in Antarctic sea ice extent over the past 50 years. Geophysical Research Letters 42, 1 (2015), 90-95.

[264] GainForest. 2021. GainForest. Retrieved from https://www.gainforest.app/.

[265] Matthias D. Galus, Marina González Vayá, Thilo Krause, and Göran Andersson. 2013. The role of electric vehicles in smart grids. Wiley Interdisciplinary Reviews: Energy and Environment 2, 4 (2013), 384-400.

[266] Sriram Ganapathi Subramanian and Mark Crowley. 2018. Combining MCTS and A3C for prediction of spatially spreading processes in forest wildfire settings. In Advances in Artificial Intelligence: 31st Canadian Conference on Artificial Intelligence, Canadian AI 2018. Springer, 285-291.

[267] Sriram Ganapathi Subramanian and Mark Crowley. 2018. Using spatial reinforcement learning to build forest wildfire dynamics models from satellite images. Frontiers in ICT 5 (2018), 6.

[268] Jim Gao. 2014. Machine learning applications for data center optimization. Retrived from https://docs.google. com/a/google.com/viewer?url=www.google.com/about/datacenters/efficiency/internal/assets/machine-learningapplicationsfor-datacenter-optimization-finalv2.pdf.

[269] T. Gasser, Céline Guivarch, K. Tachiiri, C. D. Jones, and P. Ciais. 2015. Negative emissions physically needed to keep global warming below 2C. Nature Communications 6 (2015), 7958.

[270] Massimiliano Gastaldi, Riccardo Rossi, Gregorio Gecchele, and Luca Della Lucia. 2013. Annual average daily traffic estimation from seasonal traffic counts. Procedia-Social and Behavioral Sciences 87 (2013), $279-291$.

[271] Xiou Ge, Richard T. Goodwin, Jeremy R. Gregory, Randolph E. Kirchain, Joana Maria, and Lav R. Varshney. 2019. Accelerated discovery of sustainable building materials. Preprint arXiv:1905.08222 (2019).

[272] Sylvie Geisendorf. 2018. Evolutionary climate-change modelling: A multi-agent climate-economic model. Computational Economics 52, 3 (2018), 921-951.

[273] Christian Geiß, Hannes Taubenböck, Michael Wurm, Thomas Esch, Michael Nast, Christoph Schillings, and Thomas Blaschke. 2011. Remote sensing-based characterization of settlement structures for assessing local potential of district heat. Remote Sensing 3, 7 (2011), 1447-1471.

[274] Liesje Van Gelder, Payel Das, Hans Janssen, and Staf Roels. 2014. Comparative study of metamodelling techniques in building energy simulation: Guidelines for practitioners. Simulation Modelling Practice and Theory 49 (2014), 245-257.

[275] P. Gentine, M. Pritchard, S. Rasp, G. Reinaudi, and G. Yacalis. 2018. Could machine learning break the convection parameterization deadlock? Geophysical Research Letters 45, 11 (2018), 5742-5751.

[276] Neil Gershenfeld, Stephen Samouhos, and Bruce Nordman. 2010. Intelligent infrastructure for energy efficiency. Science 327, 5969 (2010), 1086-1088.

[277] Neil Gershenfeld, Stephen Samouhos, and Bruce Nordman. 2010. Intelligent infrastructure for energy efficiency. Science 327, 5969 (2010), 1086-1088.

[278] Dimitry Gershenson, Brandon Rohrer, and Anna Lerner. 2019. A new predictive model for more accurate electrical grid mapping. Retrived from https:/code.fb.com/connectivity/electrical-grid-mapping/.

[279] Ben Gesing and D. Peterson, and S. Michelsen. 2018. Artificial intelligence in logistics: A collaborative report by DHL and IBM on implications and use cases for the logistics industry. DHL Trend Research, Troisdorf.

[280] Mohammad Sajjad Ghaemi, Bruno Agard, Martin Trépanier, and Vahid Partovi Nia. 2017. A visual segmentation method for temporal smart card data. Transportmetrica A: Transport Science 13, 5 (2017), 381-404.

[281] A. Ghanem, M. Elhenawy, M. Almannaa, H. I. Ashqar, and H. A. Rakha. 2017. Bike share travel time modeling: San Francisco bay area case study. In 2017 5th IEEE International Conference on Models and Technologies for Intelligent Transportation Systems (MT-ITS'17). 586-591.

[282] Dana M. Ghioca-Robrecht, Carol A. Johnston, and Mirela G. Tulbure. 2008. Assessing the use of multiseason QuickBird imagery for mapping invasive species in a Lake Erie coastal marsh. Wetlands 28, 4 (2008), 1028-1039.

[283] Hamed Ghoddusi, Germán G. Creamer, and Nima Rafizadeh. 2019. Machine learning in energy economics and finance: A review. Energy Economics 81 (2019), 709-727.

[284] Gianfranco Gianfrate. 2018. Designing carbon-neutral investment portfolios. In Designing a Sustainable Financial System. Springer, 151-171.

[285] Gregor Giebel and George Kariniotakis. 2017. Wind power forecasting-A review of the state of the art. In Renewable Energy Forecasting. Elsevier, 59-109.

[286] Sarah Giest. 2017. Big data analytics for mitigating carbon emissions in smart cities: Opportunities and challenges. European Planning Studies 25, 6 (2017), 941-957. 
[287] Y. Gil, S. Pierce, Hassan Babaie, Arindam Banerjee, Kirk Borne, Gary Bust, Michelle Cheatham, Imme Ebert-Uphoff, Carla Gomes, Mary Hill, John Horel, Leslie Hsu, Jim Kinter, Craig Knoblock, David Krum, Vipin Kumar, Pierre Lermusiaux, Yan Liu, Chris North, Victor Pankratius, Shanan Peters, Beth Plale, Allen Pope, Sai Ravela, Juan Restrepo, Aaron Ridley, Hanan Samet, and Shashi Shekhar. 2019. Intelligent systems for geosciences: An essential research agenda. Communications of the ACM 62, 1 (2019), 76-84.

[288] Kenneth Gillingham and James H. Stock. 2018. The cost of reducing greenhouse gas emissions. fournal of Economic Perspectives 32, 4 (2018), 53-72.

[289] Matteo Giuliani, Andrea Castelletti, Francesca Pianosi, Emanuele Mason, and Patrick M. Reed. 2015. Curses, tradeoffs, and scalable management: Advancing evolutionary multiobjective direct policy search to improve water reservoir operations. Journal of Water Resources Planning and Management 142, 2 (2015), 04015050.

[290] Edward Glaessgen and David Stargel. 2012. The digital twin paradigm for future NASA and US Air Force vehicles. In 53rd AIAA/ASME/ASCE/AHS/ASC Structures, Structural Dynamics and Materials Conference 20th AIAA/ASME/AHS Adaptive Structures Conference 14th AIAA. 1818.

[291] Mevludin Glavic. 2019. (Deep) Reinforcement learning for electric power system control and related problems: A short review and perspectives. Annual Reviews in Control 48 (2019), 22-35.

[292] Mevludin Glavic, Raphaël Fonteneau, and Damien Ernst. 2017. Reinforcement learning for electric power system decision and control: Past considerations and perspectives. IFAC-PapersOnLine 50, 1 (2017), 6918-6927.

[293] Global Thermostat. 2021. Global Thermostat. Retrieved from https://globalthermostat.com/.

[294] SCM Globe. 2015. Zara clothing company supply chain. SCM Globe.

[295] Ulrich Gnewuch, Stefan Morana, Carl Heckmann, and Alexander Maedche. 2018. Designing conversational agents for energy feedback. In International Conference on Design Science Research in Information Systems and Technology. Springer, $18-33$.

[296] Carla Gomes, Thomas Dietterich, Bistra Dilkina, Ermon Stefano, Fei Fang, Alan Farnsworth, Alan Fern, Xioali Fern, Daniel Fink, Douglas Fisher, Alexander Flecker, Daniel Freund, Angela Fuller, John Gregoire, John Hopcroft, Zico Kolter, Warren Powell, Nicole Santov, John Selker, Bart Selman, Daniel Shelcon, David Shmoys, Milind Tambe, Christopher Wood, Weng-Keen Wong, Xiaojian Wu, Steve Kelling, Yexiang Xue, Amulya Yadav, Aziz Yakubu, and Mary Lou Zeeman. 2019. Computational sustainability: Computing for a better world and a sustainable future. Communications of ACM 62, 9 (2019), 56-65.

[297] Carla P. Gomes. 2009. Computational sustainability: Computational methods for a sustainable environment, economy, and society. The Bridge 39, 4 (2009), 5-13.

[298] Carla P. Gomes, Junwen Bai, Yexiang Xue, Johan Björck, Brendan Rappazzo, Sebastian Ament, Richard Bernstein, Shufeng Kong, Santosh K. Suram, R. Bruce van Dover, and John M. Gregoire. 2019. CRYSTAL: A multi-agent AI system for automated mapping of materials' crystal structures. MRS Communications 9, 2 (2019), 1-9.

[299] Rafael Gómez-Bombarelli, Jennifer N. Wei, David Duvenaud, José Miguel Hernández-Lobato, Benjamín SánchezLengeling, Dennis Sheberla, Jorge Aguilera-Iparraguirre, Timothy D. Hirzel, Ryan P. Adams, and Alán Aspuru-Guzik. 2018. Automatic chemical design using a data-driven continuous representation of molecules. ACS Central Science 4 , 2 (2018), 268-276.

[300] H. Goosse, P. Barriat, W. Lefebvre, M. Loutre, and V. Zunz. 2008-2010. Introduction to Climate Dynamics and Climate Modeling. Cambridge University Press.

[301] Al Gore and Gavin McCormick. 2020. We Can Solve the Climate Crisis by Tracing Pollution Back to Its Sources. A New Coalition Will Make It Possible. Retrieved from https://medium.com/@algore/we-can-solve-the-climate-crisisby-tracing-pollution-back-to-its-sources-4f535f91a8dd.

[302] Ramon Granell, Colin J. Axon, and David C. H. Wallom. 2014. Predicting winning and losing businesses when changing electricity tariffs. Applied Energy 133 (2014), 298-307.

[303] Greenpeace. 2019. Oil in the Cloud: How Tech Companies are Helping Big Oil Profit from Climate Destruction. Retrived from https://www.greenpeace.org/usa/reports/oil-in-the-cloud/.

[304] Samuel Greydanus, Misko Dzamba, and Jason Yosinski. 2019. Hamiltonian neural networks. In Advances in Neural Information Processing Systems. 15379-15389.

[305] S. Griffith, S. Calisch, and L. Fraser. 2020. Rewiring America: A Field Manual for the Climate Fight. Rewiring America.

[306] G. Griffiths, N. W. Millard, S. D. McPhail, P. Stevenson, J. R. Perrett, M. Peabody, A. T. Webb, and D. T. Meldrum. 1998. Towards environmental monitoring with the Autosub autonomous underwater vehicle. In 1998 International Symposium on Underwater Technology. IEEE, 121-125.

[307] Justin Grimmer and Brandon M. Stewart. 2013. Text as data: The promise and pitfalls of automatic content analysis methods for political texts. Political Analysis 21, 3 (2013), 267-297.

[308] Simon Gruber, Ulrich Blahak, Florian Haenel, Christoph Kottmeier, Thomas Leisner, Harel Muskatel, Trude Storelvmo, and Bernhard Vogel. 2019. A process study on thinning of Arctic winter cirrus clouds with high-resolution ICON-ART simulations. fournal of Geophysical Research: Atmospheres 124, 11 (2019), 5860-5888. 
[309] G. X. Gu, C.-T. Chen, D. J. Richmond, and M. J. Buehler. 2018. Bioinspired hierarchical composite design using machine learning: Simulation, additive manufacturing, and experiment. Materials Horizons 5, 5 (2018), 939-945.

[310] Mike Gualtieri, Noel Yuhanna, Holger Kisker, Rowan Curran, Brandon Purcell, Sophia Christakis, Shreyas Warrier, and Matthew Izzi. 2016. The Forrester Wave: Big Data Streaming Analytics, Q1 2016. Forrester.com

[311] Neel Guha, Zhecheng Wang, Matt Wytock, and Arun Majumdar. 2019. Machine Learning for AC Optimal Power Flow. Retrieved from http://www.neelguha.com/opf.pdf.

[312] Chathika Gunaratne, Ivan Garibay, and Nguyen Dang. 2020. Evolutionary model discovery of causal factors behind the socio-agricultural behavior of the ancestral Pueblo. PLoS One 15, 12 (2020), e0239922.

[313] Marc Gunther. 2010. The Power of Peer Pressure in Combatting Climate Change. Retrieved from https://www. greenbiz.com/blog/2010/01/19/power-peer-pressure-combatting-climate-change.

[314] Amrita Gupta, Caleb Robinson, and Bistra Dilkina. 2018. Infrastructure resilience for climate adaptation. In $1 s t$ ACM SIGCAS Conference on Computing and Sustainable Societies. ACM, 28.

[315] Ritwik Gupta, Bryce Goodman, Nirav Patel, Ricky Hosfelt, Sandra Sajeev, Eric Heim, Jigar Doshi, Keane Lucas, Howie Choset, and Matthew Gaston. 2019. Creating xBD: A dataset for assessing building damage from satellite imagery. In IEEE Conference on Computer Vision and Pattern Recognition Workshops. 10-17.

[316] Jenny Gustavsson, Christel Cederberg, Ulf Sonesson, Robert Van Otterdijk, and Alexandre Meybeck. 2011. Global food losses and food waste. Food and Agriculture Organization of the United Nations, Rome.

[317] César G. Gutiérrez-Arriaga, Medardo Serna-González, José María Ponce-Ortega, and Mahmoud M. El-Halwagi. 2013. Multi-objective optimization of steam power plants for sustainable generation of electricity. Clean Technologies and Environmental Policy 15, 4 (2013), 551-566.

[318] Matthew Guttenberg, Shashank Sripad, and Venkatasubramanian Viswanathan. 2017. Evaluating the potential of platooning in lowering the required performance metrics of li-ion batteries to enable practical electric semi-trucks. ACS Energy Letters 2, 11 (2017), 2642-2646.

[319] H. Habibzadeh, A. Boggio-Dandry, Z. Qin, T. Soyata, B. Kantarci, and H. T. Mouftah. 2018. Soft sensing in smart cities: Handling $3 \mathrm{Vs}$ using recommender systems, machine intelligence, and data analytics. IEEE Communications Magazine 56, 2 (2018), 78-86.

[320] Leonore Haelg, Marius Waelchli, and Tobias S. Schmidt. 2018. Supporting energy technology deployment while avoiding unintended technological lock-in: A policy design perspective. Environmental Research Letters 13, 10 (2018), 104011.

[321] Sidrah Hafeez, Man Sing Wong, Hung Chak Ho, Majid Nazeer, Janet Nichol, Sawaid Abbas, Danling Tang, Kwon Ho Lee, and Lilian Pun. 2019. Comparison of machine learning algorithms for retrieval of water quality indicators in case-II waters: A case study of Hong Kong. Remote Sensing 11, 6 (2019), 617.

[322] Julian Hagenauer and Marco Helbich. 2017. A comparative study of machine learning classifiers for modeling travel mode choice. Expert Systems with Applications 78 (2017), 273-282.

[323] Gregory D. Hager, Ann Drobnis, Fei Fang, Rayid Ghani, Amy Greenwald, Terah Lyons, David C. Parkes, Jason Schultz, Suchi Saria, Stephen F. Smith, and Milind Tambe. 2019. Artificial intelligence for social good. Preprint arXiv:1901.05406 (2019).

[324] Andy Haines, R. Sari Kovats, Diarmid Campbell-Lendrum, and Carlos Corvalán. 2006. Climate change and human health: Impacts, vulnerability and public health. Public Health 120, 7 (2006), 585-596.

[325] Jee-Hoon Han, Yu-Chan Ahn, and In-Beum Lee. 2012. A multi-objective optimization model for sustainable electricity generation and CO2 mitigation (EGCM) infrastructure design considering economic profit and financial risk. Applied Energy 95 (2012), 186-195.

[326] Yafei Han. 2018. Global urban typology discovery with a latent class choice model. In Transportation Research Board 97th Annual Meeting. 5.

[327] P. A. Hancock, Illah Nourbakhsh, and Jack Stewart. 2019. On the future of transportation in an era of automated and autonomous vehicles. Proceedings of the National Academy of Sciences 116, 16 (2019), 7684-7691.

[328] Edward Hanna, Francisco J. Navarro, Frank Pattyn, Catia M. Domingues, Xavier Fettweis, Erik R. Ivins, Robert J. Nicholls, Catherine Ritz, Ben Smith, Slawek Tulaczyk, Pippa L. Whitehouse, and H. Jay Zwally. 2013. Ice-sheet mass balance and climate change. Nature 498, 7452 (2013), 51-59.

[329] M. C. Hansen, Peter Potapov, R. Moore, M. Hancher, Svetlana Turubanova, Alexandra Tyukavina, D. Thau, Stephen Stehman, Scott Goetz, Thomas Loveland, Anil Kommareddy, Alexey Egorov, L. Chini, C. O. Justice, and J. Townshend. 2013. High-Resolution global maps of 21st-century forest cover change. Science 342, 6160 (2013), 850-853.

[330] Terry Hansen and Chia-Jiu Wang. 2005. Support vector based battery state of charge estimator. fournal of Power Sources 141, 2 (2005), 351-358.

[331] Jane K. Hart and Kirk Martinez. 2006. Environmental sensor networks: A revolution in the earth system science? Earth-Science Reviews 78, 3-4 (2006), 177-191. 
[332] Jason Hartford, Greg Lewis, Kevin Leyton-Brown, and Matt Taddy. 2017. Deep IV: A flexible approach for counterfactual prediction. In 34th International Conference on Machine Learning. JMLR.org, 1414-1423.

[333] Fouad Hasan, Amin Kargarian, and Ali Mohammadi. 2020. A survey on applications of machine learning for optimal power flow. In 2020 IEEE Texas Power and Energy Conference (TPEC'20). IEEE, 1-6.

[334] H. Hassine, Maher Barkallah, and A. Bellacicco. 2015. Multi objective optimization for sustainable manufacturing, application in turning. International fournal of Simulation Modelling 14, 1 (2015), 98-109.

[335] Paul Hawken. 2015. Drawdown: The Most Comprehensive Plan Ever Proposed to Reverse Global Warming. Penguin Books.

[336] Troy R. Hawkins, Bhawna Singh, Guillaume Majeau-Bettez, and Anders Hammer Strømman. 2013. Comparative environmental life cycle assessment of conventional and electric vehicles. Fournal of Industrial Ecology 17, 1 (2013), 53-64.

[337] Susan Helper, Raphael Martins, and Robert Seamans. 2019. Who profits from industry 4.0? Theory and evidence from the automotive industry. NYU Stern School of Business.

[338] Peter Henderson, Riashat Islam, Philip Bachman, Joelle Pineau, Doina Precup, and David Meger. 2018. Deep reinforcement learning that matters. In 32nd AAAI Conference on Artificial Intelligence.

[339] André Henn, Christoph Römer, Gerhard Gröger, and Lutz Plümer. 2012. Automatic classification of building types in 3D city models. GeoInformatica 16, 2 (2012), 281-306.

[340] Miguel A. Hernán, John Hsu, and Brian Healy. 2019. A second chance to get causal inference right: A classification of data science tasks. Chance 32, 1 (2019), 42-49.

[341] Edgar G. Hertwich, Saleem Ali, Luca Ciacci, Tomer Fishman, Niko Heeren, Eric Masanet, Farnaz Nojavan Asghari, Elsa Olivetti, Stefan Pauliuk, Qingshi Tu, and Paul Wolfram. 2019. Material efficiency strategies to reducing greenhouse gas emissions associated with buildings, vehicles, and electronics-a review. Environmental Research Letters 14, 4 (2019), 043004.

[342] Matthew G. Hethcoat, David P. Edwards, Joao M. B. Carreiras, Robert G. Bryant, Filipe M. Franca, and Shaun Quegan. 2019. A machine learning approach to map tropical selective logging. Remote Sensing of Environment 221 (2019), 569582.

[343] Michael K. Hidrue and George R. Parsons. 2015. Is there a near-term market for vehicle-to-grid electric vehicles? Applied Energy 151 (2015), 67-76.

[344] Christian Hilbe, Štěpán Šimsa, Krishnendu Chatterjee, and Martin A. Nowak. 2018. Evolution of cooperation in stochastic games. Nature 559, 7713 (2018), 246-249.

[345] David J. Hill and Barbara S. Minsker. 2010. Anomaly detection in streaming environmental sensor data: A data-driven modeling approach. Environmental Modelling \& Software 25, 9 (2010), 1014-1022.

[346] Eric Hittinger and Paulina Jaramillo. 2019. Internet of things: Energy boon or bane? Science 364, 6438 (2019), $326-328$.

[347] Eric S. Hittinger and Inês M. L. Azevedo. 2015. Bulk energy storage increases United States electricity system emissions. Environmental Science \& Technology 49, 5 (2015), 3203-3210.

[348] Hung Chak Ho, Anders Knudby, Paul Sirovyak, Yongming Xu, Matus Hodul, and Sarah B. Henderson. 2014. Mapping maximum urban air temperature on hot summer days. Remote Sensing of Environment 154 (2014), 38-45.

[349] Joseph Holden, P. J. Chapman, and J. C. Labadz. 2004. Artificial drainage of peatlands: Hydrological and hydrochemical process and wetland restoration. Progress in Physical Geography 28, 1 (2004), 95-123.

[350] Geoffrey Holmes and David W. Keith. 2012. An air-liquid contactor for large-scale capture of CO2 from air. Philosophical Transactions of the Royal Society A: Mathematical, Physical and Engineering Sciences 370, 1974 (2012), 4380-4403.

[351] Tao Hong and Shu Fan. 2016. Probabilistic electric load forecasting: A tutorial review. International fournal of Forecasting 32, 3 (2016), 914-938.

[352] Nathaniel Horner, Inês Azevedo, Doug Sicker, and Yuvraj Agarwal. 2016. Dynamic data center load response to variability in private and public electricity costs. In 2016 IEEE International Conference on Smart Grid Communications (SmartGridComm'16). IEEE, 80-85.

[353] Noriko Hosonuma, Martin Herold, Veronique De Sy, Ruth S. De Fries, Maria Brockhaus, Louis Verchot, Arild Angelsen, and Erika Romijn. 2012. An assessment of deforestation and forest degradation drivers in developing countries. Environmental Research Letters 7, 4 (2012).

[354] Frederic Hourdin, Thorsten Mauritsen, Andrew Gettelman, Jean-Christophe Golaz, Venkatramani Balaji, Qingyun Duan, Doris Folini, Duoying Ji, Daniel Klocke, Yun Qian, Florian Rauser, Catherine Rio, Lorenzo Tomassini, Masahiro Watanabe, and Daniel Williamson. 2017. The art and science of climate model tuning. Bulletin of the American Meteorological Society 98, 3 (2017), 589-602.

[355] Rachel M. Houtman, Claire A. Montgomery, Aaron R. Gagnon, David E. Calkin, Thomas G. Dietterich, Sean McGregor, and Mark Crowley. 2013. Allowing a wildfire to burn: Estimating the effect on future fire suppression costs. International fournal of Wildland Fire 22, 7 (2013), 871-882. 
[356] Isabel Hovdahl. 2019. On the use of machine learning for causal inference in climate economics. Working Papers No. 05/2019, Centre for Applied Macro- and Petroleum economics (CAMP), BI Norwegian Business School.

[357] Qinran Hu and Fangxing Li. 2013. Hardware design of smart home energy management system with dynamic price response. IEEE Transactions on Smart Grid 4, 4 (2013), 1878-1887.

[358] Xiaosong Hu, Shengbo Eben Li, and Yalian Yang. 2016. Advanced machine learning approach for lithium-ion battery state estimation in electric vehicles. IEEE Transactions on Transportation electrification 2, 2 (2016), 140-149.

[359] Bohao Huang, Jichen Yang, Artem Streltsov, Kyle Bradbury, Leslie M. Collins, and Jordan Malof. 2021. GridTracer: Automatic mapping of power grids using deep learning and overhead imagery. In IEEE fournal of Selected Topics in Applied Earth Observations and Remote Sensing. DOI : 10.1109/JSTARS.2021.3124519

[360] David Humphreys, A. Kupresanin, M. D. Boyer, J. Canik, C. S. Chang, E. C. Cyr, R. Granetz, J. Hittinger, E. Kolemen, E. Lawrence, et al. 2020. Advancing fusion with machine learning research needs workshop report. fournal of Fusion Energy 39, 4 (2020), 123-155.

[361] R. W. Hut, N. C. van de Giesen, and J. S. Selker. 2012. The TAHMO project: Designing an unconventional weather station. In EGU General Assembly Conference Abstracts. Vol. 14. 8963.

[362] Duy Huynh and Nathalie Neptune. 2018. Annotation automatique d'images: Le cas de la déforestation. In Actes de la conférence Traitement Automatique de la Langue Naturelle (TALN'18). 101.

[363] Jessica Hwang, Paulo Orenstein, Judah Cohen, Karl Pfeiffer, and Lester Mackey. 2019. Improving subseasonal forecasting in the western U.S. with machine learning. In 25th ACM SIGKDD International Conference on Knowledge Discovery \& Data Mining.

[364] Michael Hyland, Zihan Hong, Helen Karla Ramalho de Farias Pinto, and Ying Chen. 2018. Hybrid cluster-regression approach to model bikeshare station usage. Transportation Research Part A: Policy and Practice 115 (2018), 71-89.

[365] Ana Iglesias, Paloma Martínez, Ricardo Aler, and Fernando Fernández. 2009. Learning teaching strategies in an adaptive and intelligent educational system through reinforcement learning. Applied Intelligence 31, 1 (2009), 89-106.

[366] IHE Delft Institute for Water Education. 2019. The Water, Peace and Security Partnership. Retrieved from https:// www.un-ihe.org/water-peace-and-security-partnership.

[367] Rositsa T. Ilieva and Timon McPhearson. 2018. Social-media data for urban sustainability. Nature Sustainability 1, 10 (2018), 553

[368] Muhammad Imran, Carlos Castillo, Fernando Diaz, and Sarah Vieweg. 2015. Processing social media messages in mass emergency: A survey. ACM Computing Surveys 47, 4 (2015), 67.

[369] Instituto Nacional de Pesquisas Espaciais. 2020. Portal TerraBrasilis. Retrieved from http://terrabrasilis.dpi.inpe.br/ en/home-page/.

[370] IPCC. 2014. Climate Change 2014: Mitigation of Climate Change. Contribution of Working Group III to the Fifth Assessment Report of the Intergovernmental Panel on Climate Change. O. Edenhofer, R. Pichs-Madruga, Y. Sokona, E. Farahani, S. Kadner, K. Seyboth, A. Adler, I. Baum, S. Brunner, P. Eickemeier, B. Kriemann, J. Savolainen, S. Schlömer, C. von Stechow, T. Zwickel, J. C. Minx (Eds.). Intergovernmental Panel on Climate Change.

[371] IPCC. 2014. Climate Change 2014: Synthesis Report. Contribution of Working Groups I, II and III to the Fifth Assessment Report of the Intergovernmental Panel on Climate Change. Core Writing Team, R. K. Pachauri and L. A. Meyer (Eds.). Intergovernmental Panel on Climate Change.

[372] IPCC. 2018. Global warming of $1.5^{\circ} \mathrm{C}$. An IPCC special report on the impacts of global warming of $1.5^{\circ} \mathrm{C}$ above preindustrial levels and related global greenhouse gas emission pathways, in the context of strengthening the global response to the threat of climate change, sustainable development, and efforts to eradicate poverty. Intergovernmental Panel on Climate Change.

[373] Christopher Irrgang, Niklas Boers, Maike Sonnewald, Elizabeth A. Barnes, Christopher Kadow, Joanna Staneva, and Jan Saynisch-Wagner. 2021. Towards neural Earth system modelling by integrating artificial intelligence in Earth system science. Nature Machine Intelligence 3 (2021), 667-674.

[374] Peter Irvine, Kerry Emanuel, Jie He, Larry W. Horowitz, Gabriel Vecchi, and David Keith. 2019. Halving warming with idealized solar geoengineering moderates key climate hazards. Nature Climate Change 9 (2019), 295-299.

[375] Peter J. Irvine, Ben Kravitz, Mark G. Lawrence, and Helene Muri. 2016. An overview of the Earth system science of solar geoengineering. Wiley Interdisciplinary Reviews: Climate Change 7, 6 (2016), 815-833.

[376] Sibren Isaacman, Vanessa Frias-Martinez, Lingzi Hong, and Enrique Frias-Martinez. 2017. Climate change induced migrations from a cell phone perspective. NetMob (2017), 46.

[377] Srinivasan Iyengar, Stephen Lee, Daniel Sheldon, and Prashant Shenoy. 2018. Solarclique: Detecting anomalies in residential solar arrays. In 1st ACM SIGCAS Conference on Computing and Sustainable Societies. ACM, 38.

[378] Daniel J. Jacob, Alexander J. Turner, Joannes D. Maasakkers, Jianxiong Sheng, Kang Sun, Xiong Liu, Kelly Chance, Ilse Aben, Jason McKeever, and Christian Frankenberg. 2016. Satellite observations of atmospheric methane and their value for quantifying methane emissions. Atmospheric Chemistry and Physics 16, 22 (2016), 14371-14396. 
[379] Alexandre Jacquillat and Amedeo R. Odoni. 2018. A roadmap toward airport demand and capacity management. Transportation Research Part A: Policy and Practice 114 (2018), 168-185.

[380] Anubhav Jain, Shyue Ping Ong, Geoffroy Hautier, Wei Chen, William Davidson Richards, Stephen Dacek, Shreyas Cholia, Dan Gunter, David Skinner, Gerbrand Ceder, and Kristin A. Persson. 2013. Commentary: The Materials Project: A materials genome approach to accelerating materials innovation. Apl Materials 1, 1 (2013), 011002.

[381] Vipin Jain, Ashlesh Sharma, and Lakshminarayanan Subramanian. 2012. Road traffic congestion in the developing world. In 2nd ACM Symposium on Computing for Development. ACM, 11.

[382] Mahdi Jamei, Letif Mones, Alex Robson, Lyndon White, James Requeima, and Cozmin Ududec. 2019. Metaoptimization of optimal power flow. In ICML Workshop on Climate Change: How Can AI Help?

[383] Ali Jamshidi, Siamak Hajizadeh, Zhou Su, Meysam Naeimi, Alfredo Núñez, Rolf Dollevoet, Bart De Schutter, and Zili Li. 2018. A decision support approach for condition-based maintenance of rails based on big data analysis. Transportation Research Part C: Emerging Technologies 95 (2018), 185-206.

[384] Vijay Manikandan Janakiraman, XuanLong Nguyen, and Dennis Assanis. 2016. Stochastic gradient based extreme learning machines for stable online learning of advanced combustion engines. Neurocomputing 177 (2016), 304-316.

[385] Natasha Jaques, Angeliki Lazaridou, Edward Hughes, Çaglar Gülçehre, Pedro A. Ortega, D. J. Strouse, Joel Z. Leibo, and Nando de Freitas. In Freitas Proceedings of the 36th International Conference on Machine Learning.

[386] Marc Jaxa-Rozen and Jan Kwakkel. 2018. Tree-based ensemble methods for sensitivity analysis of environmental models: A performance comparison with Sobol and Morris techniques. Environmental Modelling \& Software 107 (2018), 245-266.

[387] Feng Jia, Yaguo Lei, Jing Lin, Xin Zhou, and Na Lu. 2016. Deep neural networks: A promising tool for fault characteristic mining and intelligent diagnosis of rotating machinery with massive data. Mechanical Systems and Signal Processing 72 (2016), 303-315.

[388] Huaiguang Jiang and Yingchen Zhang. 2016. Short-term distribution system state forecast based on optimal synchrophasor sensor placement and extreme learning machine. In 2016 IEEE Power and Energy Society General Meeting (PESGM'16). IEEE, 1-5.

[389] M. Jiang, B. Gallagher, J. Kallman, and D. Laney. 2016. A supervised learning framework for arbitrary LagrangianEulerian simulations. In 15th IEEE International Conference on Machine Learning and Applications (ICMLA'16). Anaheim, CA.

[390] Qiling Jiang, Liujuan Cao, Ming Cheng, Cheng Wang, and Jonathan Li. 2015. Deep neural networks-based vehicle detection in satellite images. In 2015 International Symposium on Bioelectronics and Bioinformatics (ISBB'15). IEEE, 184-187.

[391] Shan Jiang, Gaston A. Fiore, Yingxiang Yang, Joseph Ferreira Jr, Emilio Frazzoli, and Marta C. González. 2013. A review of urban computing for mobile phone traces: Current methods, challenges and opportunities. In 2nd ACM SIGKDD International Workshop on Urban Computing. ACM, 2.

[392] David Jiménez, Sara Hernández, Jesús Fraile-Ardanuy, Javier Serrano, Rubén Fernández, and Federico Álvarez. 2018. Modelling the effect of driving events on electrical vehicle energy consumption using inertial sensors in smartphones. Energies 11, 2 (2018), 412

[393] Xin Jin, Kyri Baker, Dane Christensen, and Steven Isley. 2017. Foresee: A user-centric home energy management system for energy efficiency and demand response. Applied Energy 205 (2017), 1583-1595.

[394] Michael A. Johansson, Nicholas G. Reich, Aditi Hota, John S. Brownstein, and Mauricio Santillana. 2016. Evaluating the performance of infectious disease forecasts: A comparison of climate-driven and seasonal dengue forecasts for Mexico. Scientific Reports 6 (2016), 33707.

[395] Srećko Joksimović, Oleksandra Poquet, Vitomir Kovanović, Nia Dowell, Caitlin Mills, Dragan Gašević, Shane Dawson, Arthur C. Graesser, and Christopher Brooks. 2018. How do we model learning at scale? A systematic review of research on MOOCs. Review of Educational Research 88, 1 (2018), 43-86.

[396] Andy Jones, Jim Haywood, and Olivier Boucher. 2009. Climate impacts of geoengineering marine stratocumulus clouds. fournal of Geophysical Research: Atmospheres 114, D10 (2009).

[397] Charlotte Jones, Donald W. Hine, and Anthony D. G. Marks. 2017. The future is now: Reducing psychological distance to increase public engagement with climate change. Risk Analysis 37, 2 (2017), 331-341.

[398] Christopher Jones and Daniel M. Kammen. 2014. Spatial distribution of US household carbon footprints reveals suburbanization undermines greenhouse gas benefits of urban population density. Environmental Science \& Technology 48, 2 (2014), 895-902.

[399] Christopher M. Jones and Daniel M. Kammen. 2011. Quantifying carbon footprint reduction opportunities for US households and communities. Environmental Science \& Technology 45, 9 (2011), 4088-4095.

[400] Hans Joosten, Marja-Liisa Tapio-Biström, and Susanna Tol. 2012. Peatlands: Guidance for Climate Change Mitigation through Conservation, Rehabilitation and Sustainable Use. Food and Agriculture Organization of the United Nations.

[401] Lucas N. Joppa. 2017. The case for technology investments in the environment. Nature 552, 7685 (2017), 325-328. 
[402] Romain Juban, Henrik Ohlsson, Mehdi Maasoumy, Louis Poirier, and J. Zico Kolter. 2016. A multiple quantile regression approach to the wind, solar, and price tracks of GEFCom2014. International fournal of Forecasting 32, 3 (2016), 1094-1102.

[403] Lynn Helena Kaack. 2019. Challenges and Prospects for Data-Driven Climate Change Mitigation. Ph.D. Dissertation. Carnegie Mellon University, Pittsburgh, PA.

[404] Lynn H. Kaack, Jay Apt, M. Granger Morgan, and Patrick McSharry. 2017. Empirical prediction intervals improve energy forecasting. Proceedings of the National Academy of Sciences 114, 33 (2017), 8752-8757.

[405] Lynn H. Kaack, George H. Chen, and M. Granger Morgan. 2019. Truck traffic monitoring with satellite images. In 2nd ACM SIGCAS Conference on Computing and Sustainable Societies (COMPASS'19). ACM, New York, NY, 155-164.

[406] Lynn H. Kaack, Priya L. Donti, Emma Strubell, and David Rolnick. 2020. Artificial intelligence and climate change: Opportunities, considerations, and policy levers to align AI with climate change goals. Retrived from https://eu.boell. org/en/2020/12/03/artificial-intelligence-and-climate-change.

[407] Lynn H. Kaack, Parth Vaishnav, M. Granger Morgan, Inês L. Azevedo, and Srijana Rai. 2018. Decarbonizing intraregional freight systems with a focus on modal shift. Environmental Research Letters 13, 8 (2018), 083001.

[408] Namwoo Kang, Fred M. Feinberg, and Panos Y. Papalambros. 2016. Autonomous electric vehicle sharing system design. fournal of Mechanical Design 139, 1 (2016), 011402-011402-10.

[409] Emre C. Kara, Ciaran M. Roberts, Michaelangelo Tabone, Lilliana Alvarez, Duncan S. Callaway, and Emma M. Stewart. 2018. Disaggregating solar generation from feeder-level measurements. Sustainable Energy, Grids and Networks 13 (2018), 112-121.

[410] Stavros Karagiannopoulos, Petros Aristidou, and Gabriela Hug. 2019. Data-driven local control design for active distribution grids using off-line optimal power flow and machine learning techniques. IEEE Transactions on Smart Grid 10, 6 (2019), 6461-6471.

[411] Stavros Karagiannopoulos, Roel Dobbe, Petros Aristidou, Duncan Callaway, and Gabriela Hug. 2019. Data-driven control design schemes in active distribution grids: Capabilities and challenges. In 2019 IEEE PowerTech Conference. IEEE.

[412] K. Kashinath, M. Mustafa, A. Albert, J. L. Wu, C. Jiang, S. Esmaeilzadeh, K. Azizzadenesheli, R. Wang, A. Chattopadhyay, A. Singh, and A. Manepalli. 2021. Physics-informed machine learning: Case studies for weather and climate modelling. Philosophical Transactions of the Royal Society A 379, 2194 (2021), 20200093.

[413] Julian Kates-Harbeck, Alexey Svyatkovskiy, and William Tang. 2019. Predicting disruptive instabilities in controlled fusion plasmas through deep learning. Nature 568 (2019), 526-531.

[414] Steven K. Kauwe, Trevor David Rhone, and Taylor D. Sparks. 2019. Data-driven studies of li-ion-battery materials. Crystals 9, 1 (2019), 54

[415] J. Kay, C. Deser, A. Phillips, A. Mai, C. Hannay, G. Strand, J. M. Arblaster, S. C. Bates, G. Danabasoglu, J. Edwards, M. Holland, P. Kushner, J.-F. Lamarque, D. Lawrence, K. Lindsay, A. Middleton, E. Munoz, R. Neale, K. Oleson, L. Polvani, and M. Vertenstein. 2015. The Community Earth System Model (CESM) Large Ensemble project: A community resource for studying climate change in the presence of internal climate variability. Bulletin of the American Meteorological Society 96, 8 (2015), 1333-1349.

[416] Rubaiat Habib Kazi, Tovi Grossman, Hyunmin Cheong, Ali Hashemi, and George W. Fitzmaurice. 2017. DreamSketch: Early stage 3D design explorations with sketching and generative design. In 30th Annual ACM Symposium on User Interface Software and Technology. ACM, 401-414.

[417] Hussain Kazmi, Fahad Mehmood, Stefan Lodeweyckx, and Johan Driesen. 2018. Gigawatt-hour scale savings on a budget of zero: Deep reinforcement learning based optimal control of hot water systems. Energy 144 (2018), 159-168.

[418] David Keith and Peter Irvine. 2018. The science and technology of solar geoengineering: A compact summary. In Workshop on Governance of the Deployment of Solar Geoengineering.

[419] David W. Keith. 2000. Geoengineering the climate: History and prospect. Annual Review of Energy and the Environment 25, 1 (2000), 245-284.

[420] David W. Keith. 2017. Toward a responsible solar geoengineering research program. Issues in Science and Technology 33, 3 (2017), 71-77.

[421] Alexander Kell, A. Stephen McGough, and Matthew Forshaw. 2018. Segmenting residential smart meter data for short-term load forecasting. In 9th International Conference on Future Energy Systems. ACM, 91-96.

[422] David L. Kelly and Charles D. Kolstad. 1999. Integrated assessment models for climate change control. In International Yearbook of Environmental and Resource Economics 1999/2000. Edward Elgar, 171-197.

[423] Jack Kelly and William Knottenbelt. 2015. Neural NILM: Deep neural networks applied to energy disaggregation. In 2nd ACM International Conference on Embedded Systems for Energy-Efficient Built Environments (BuildSys'15). ACM, New York, NY, 55-64.

[424] Jeffrey F. Kelly, Kyle G. Horton, Phillip M. Stepanian, Kirsten M. de Beurs, Todd Fagin, Eli S. Bridge, and Phillip B. Chilson. 2016. Novel measures of continental-scale avian migration phenology related to proximate environmental cues. Ecosphere 7, 9 (2016). 
[425] Charles D. Kerchner and William S. Keeton. 2015. California's regulatory forest carbon market: Viability for northeast landowners. Forest Policy and Economics 50 (2015), 70-81.

[426] Hannah Kerner, Catherine Nakalembe, and Inbal Becker-Reshef. 2020. Field-level crop type classification with k nearest neighbors: A baseline for a new Kenya smallholder dataset. In ICLR Workshop on Tackling Climate Change with Machine Learning.

[427] Hannah Kerner, Gabriel Tseng, Inbal Becker-Reshef, Catherine Nakalembe, Brian Barker, Blake Munshell, Madhava Paliyam, and Mehdi Hosseini. 2020. Rapid response crop maps in data sparse regions. In KDD Workshop on Humanitarian Mapping.

[428] Kevin P. Gibbons. 2014. Hyperspectral Imaging What is it? How does it work? Technical Report. Retrived from https:// www.techbriefs.com/component/content/article/tb/features/application-briefs/19507.

[429] Ahsan Raza Khan, Anzar Mahmood, Awais Safdar, Zafar A. Khan, and Naveed Ahmed Khan. 2016. Load forecasting, dynamic pricing and DSM in smart grid: A review. Renewable and Sustainable Energy Reviews 54 (2016), 1311-1322.

[430] Shahidur R. Khandker, Douglas F. Barnes, Hussain Samad, and Nguyen Huu Minh. 2009. Welfare Impacts of Rural Electrification: Evidence from Vietnam. The World Bank.

[431] Shahidur R. Khandker, Douglas F. Barnes, and Hussain A. Samad. 2009. Welfare Impacts of Rural Electrification: A Case Study from Bangladesh. The World Bank.

[432] Fazel Khayatian, Luca Sarto, et al. 2017. Building energy retrofit index for policy making and decision support at regional and national scales. Applied Energy 206 (2017), 1062-1075.

[433] Muin J. Khoury, Tram Kim Lam, John P. A. Ioannidis, Patricia Hartge, Margaret R. Spitz, Julie E. Buring, Stephen J. Chanock, Robert T. Croyle, Katrina A. Goddard, Geoffrey S. Ginsburg, Zdenko Herceg, Robert A. Hiatt, Robert N Hoover, David J. Hunter, Barnet S. Kramer, Michael S. Lauer, Jeffrey A. Meyerhardt, Olufunmilayo I. Olopade, Julie R. Palmer, Thomas A. Sellers, Daniela Seminara, David F. Ransohoff, Timothy R. Rebbeck, Georgia Tourassi, Deborah M. Winn, Ann Zauber, and Sheri D. Schully. 2013. Transforming epidemiology for 21st century medicine and public health. Cancer Epidemiology and Prevention Biomarkers 22, 4 (2013), 508-516.

[434] Jaewoo Kim, Meeyoung Cha, and Jong Gun Lee. 2017. Nowcasting commodity prices using social media. Peerf Computer Science 3 (2017), e126.

[435] Woohyun Kim and James E. Braun. 2012. Evaluation of the impacts of refrigerant charge on air conditioner and heat pump performance. International fournal of Refrigeration 35, 7 (2012), 1805-1814.

[436] Andrei P. Kirilenko and Svetlana O. Stepchenkova. 2014. Public microblogging on climate change: One year of Twitter worldwide. Global Environmental Change 26 (2014), 171-182.

[437] Daniel Sadi Kirschen and Goran Strbac. 2004. Fundamentals of Power System Economics. Vol. 1. Wiley Online Library.

[438] Kirk R. Klausmeyer and M. Rebecca Shaw. 2009. Climate change, habitat loss, protected areas and the climate adaptation potential of species in Mediterranean ecosystems worldwide. PloS One 4, 7 (2009), e6392.

[439] David Klenert, Linus Mattauch, Emmanuel Combet, Ottmar Edenhofer, Cameron Hepburn, Ryan Rafaty, and Nicholas Stern. 2018. Making carbon pricing work for citizens. Nature Climate Change 8, 8 (2018), 669-677.

[440] Nikhil Kumar Koditala and Purnendu Shekar Pandey. 2018. Water quality monitoring system using IoT and machine learning. In 2018 International Conference on Research in Intelligent and Computing in Engineering (RICE'18). IEEE, 1-5.

[441] Kenneth R. Koedinger, Emma Brunskill, Ryan S. J. D. Baker, Elizabeth A. McLaughlin, and John Stamper. 2013. New potentials for data-driven intelligent tutoring system development and optimization. AI Magazine 34, 3 (2013), 27-41.

[442] Julian Kölbel, Markus Leippold, Jordy Rillaerts, and Qian Wang. 2020. Does the CDS market reflect regulatory climate risk disclosures? Working Paper, University of Zurich.

[443] J. Zico Kolter, Siddharth Batra, and Andrew Y. Ng. 2010. Energy disaggregation via discriminative sparse coding. In Advances in Neural Information Processing Systems. 1153-1161.

[444] J. Zico Kolter and Joseph Ferreira. 2011. A large-scale study on predicting and contextualizing building energy usage. In 25th AAAI Conference on Artificial Intelligence.

[445] J. Zico Kolter and Tommi Jaakkola. 2012. Approximate inference in additive factorial HMMs with application to energy disaggregation. In 15th International Conference on Artificial Intelligence and Statistics. 1472-1482.

[446] Venkata Shashank Konduri, Jitendra Kumar, Forrest Hoffman, Udit Bhatia, Tarik Gouthier, and Auroop Ganguly. 2019. Physics-Guided Data Science for Food Security and Climate. In KDD Feed Workshop 2019. Retrived from https:// drive.google.com/file/d/1dOGIjbgMGPTpnFIvimpOlPvz28BMw2_Q/view.

[447] Ioannis C. Konstantakopoulos, Andrew R. Barkan, Shiying He, Tanya Veeravalli, Huihan Liu, and Costas Spanos. 2019. A deep learning and gamification approach to improving human-building interaction and energy efficiency in smart infrastructure. Applied Energy 237 (2019), 810-821.

[448] Constantine E. Kontokosta and Christopher Tull. 2017. A data-driven predictive model of city-scale energy use in buildings. Applied Energy 197 (2017), 303-317. 
[449] Robert E. Kopp, Robert M. Deconto, Daniel A. Bader, Carling C. Hay, M. Radley, Scott Kulp, Michael Oppenheimer, David Pollard, and Benjamin H. Strauss. 2017. Evolving understanding of Antarctic ice-sheet physics and ambiguity in probabilistic sea-level projections. Earth's Future 5, 12 (2017), 1217-1233.

[450] Branko Kosovic, Sue Ellen Haupt, Daniel Adriaansen, Stefano Alessandrini, Gerry Wiener, Luca Delle Monache, Yubao Liu, Seth Linden, Tara Jensen, William Cheng, Marcia Politovich, and Paul Prestopnik. 2020. A comprehensive wind power forecasting system integrating artificial intelligence and numerical weather prediction. Energies 13, 6 (2020), 1372.

[451] Bartosz Krawczyk, Leandro L. Minku, João Gama, Jerzy Stefanowski, and Michał Woźniak. 2017. Ensemble learning for data stream analysis: A survey. Information Fusion 37 (2017), 132-156.

[452] J. F. Kreider, D. E. Claridge, P. Curtiss, R. Dodier, J. S. Haberl, and M. Krarti. 1995. Building energy use prediction and system identification using recurrent neural networks. Fournal of Solar Energy Engineering 117, 3 (1995), 161-166.

[453] Noemi Kreif and Karla DiazOrdaz. 2019. Machine learning in policy evaluation: New tools for causal inference. In Oxford Research Encyclopedia of Economics and Finance. OUP.

[454] Robert Krile, Fred Todt, and Jeremy Schroeder. 2016. Assessing Roadway Traffic Count Duration and Frequency Impacts on Annual Average Daily Traffic Estimation. Technical Report FHWA-PL-16-012. Federal Highway Administration, Washington, D.C.

[455] Scott A. Kulp and Benjamin H. Strauss. 2019. New elevation data triple estimates of global vulnerability to sea-level rise and coastal flooding. Nature Communications 10, 1 (2019), 1-12.

[456] Thorsten Kurth, Sean Treichler, Joshua Romero, Mayur Mudigonda, Nathan Luehr, Everett Phillips, Ankur Mahesh, Michael Matheson, Jack Deslippe, Massimiliano Fatica, Prabhat, and Michael Houston. 2018. Exascale deep learning for climate analytics. In International Conference for High Performance Computing, Networking, Storage, and Analysis (SC'18). IEEE Press, Piscataway, NJ, Article 51, 12 pages.

[457] Nataliia Kussul, Mykola Lavreniuk, Sergii Skakun, and Andrii Shelestov. 2017. Deep learning classification of land cover and crop types using remote sensing data. IEEE Geoscience and Remote Sensing Letters 14, 5 (2017), 778-782.

[458] Corentin Kuster, Yacine Rezgui, and Monjur Mourshed. 2017. Electrical load forecasting models: A critical systematic review. Sustainable Cities and Society 35 (2017), 257-270.

[459] Alexandre Lacoste, Alexandra Luccioni, Victor Schmidt, and Thomas Dandres. 2019. Quantifying the carbon emissions of machine learning. Preprint arXiv:1910.09700 (2019).

[460] Jesus Lago, Fjo De Ridder, and Bart De Schutter. 2018. Forecasting spot electricity prices: Deep learning approaches and empirical comparison of traditional algorithms. Applied Energy 221 (2018), 386-405.

[461] Y.-C. Lai, C. P. L. Barkan, J. Drapa, N. Ahuja, J. M. Hart, P. J. Narayanan, C. V. Jawahar, A. Kumar, L. R. Milhon, and M. P. Stehly. 2007. Machine vision analysis of the energy efficiency of intermodal freight trains. Institution of Mechanical Engineers, Part F: Journal of Rail and Rapid Transit 221, 3 (2007), 353-364.

[462] Milena Lakicevic, Zorica Srdjevic, Bojan Srdjevic, and Miodrag Zlatic. 2014. Decision making in urban forestry by using approval voting and multicriteria approval method (case study: Zvezdarska forest, Belgrade, Serbia). Urban Forestry \& Urban Greening 13, 1 (2014), 114-120.

[463] Valliappa Lakshmanan and Travis Smith. 2010. An objective method of evaluating and devising storm-tracking algorithms. Weather and Forecasting 25, 2 (2010), 701-709.

[464] Balaji Lakshminarayanan, Alexander Pritzel, and Charles Blundell. 2017. Simple and scalable predictive uncertainty estimation using deep ensembles. In Advances in Neural Information Processing Systems.

[465] Kara D. Lamb and Pierre Gentine. 2021. Zero-shot learning of aerosol optical properties with graph neural networks. arXiv preprint arXiv:2107.10197 (2021).

[466] William F. Lamb, Felix Creutzig, Max W. Callaghan, and Jan C. Minx. 2019. Learning about urban climate solutions from case studies. Nature Climate Change 9 (2019), 279-287.

[467] Vasileios Lampos, Tijl De Bie, and Nello Cristianini. 2010. Flu detector-tracking epidemics on Twitter. In foint European Conference on Machine Learning and Knowledge Discovery in Databases. Springer, 599-602.

[468] D. J. Lary. 2010. Artificial intelligence in geoscience and remote sensing. In Aerospace Technologies Advancements. BoD-Books on Demand.

[469] David J. Lary, Amir H. Alavi, Amir H. Gandomi, and Annette L. Walker. 2015. Machine learning in geosciences and remote sensing. Geoscience Frontiers 7 (2015), 3-10.

[470] D. J. Lary, G. K. Zewdie, X. Liu, D. Wu, E. Levetin, Allee R. J., Nabin Malakar, A. Walker, H. Mussa, Mannino A., and Aurin D. 2018. Machine learning for applications for Earth observation. Earth Observation Open Science and Innovation. Springer, 165-218.

[471] Jörg Lässig, Kristian Kersting, and Katharina Morik. 2016. Computational Sustainability. Vol. 645. Springer.

[472] David Lazer, Ryan Kennedy, Gary King, and Alessandro Vespignani. 2014. The parable of Google Flu: Traps in big data analysis. Science 343, 6176 (2014), 1203-1205. 
[473] Gregory S. Ledva, Laura Balzano, and Johanna L. Mathieu. 2018. Real-time energy disaggregation of a distribution feeder's demand using online learning. IEEE Transactions on Power Systems 33, 5 (2018), 4730-4740.

[474] Hanbong Lee, Waqar Malik, Bo Zhang, Balaji Nagarajan, and Yoon C. Jung. 2015. Taxi time prediction at Charlotte Airport using fast-time simulation and machine learning techniques. In 15th AIAA Aviation Technology, Integration, and Operations Conference. 2272.

[475] Hyun-Rok Lee and Taesik Lee. 2019. Improved cooperative multi-agent reinforcement learning algorithm augmented by mixing demonstrations from centralized policy. In 18th International Conference on Autonomous Agents and MultiAgent Systems (AAMAS'19). International Foundation for Autonomous Agents and Multiagent Systems, Richland, SC, $1089-1098$.

[476] Kenneth Leerbeck, Peder Bacher, Rune Grønborg Junker, Goran Goranović, Olivier Corradi, Razgar Ebrahimy, Anna Tveit, and Henrik Madsen. 2020. Short-term forecasting of CO2 emission intensity in power grids by machine learning. Applied Energy 277 (2020), 115527.

[477] Johanna Lehne and Felix Preston. 2018. Making Concrete Change, Innovation in Low-carbon Cement and Concrete. Chatham House Report, Energy Enivronment and Resources Department: London, UK, 1-66.

[478] Jiaming Li, John K. Ward, Jingnan Tong, Lyle Collins, and Glenn Platt. 2016. Machine learning for solar irradiance forecasting of photovoltaic system. Renewable Energy 90 (2016), 542-553.

[479] Lianfa Li. 2019. Geographically weighted machine learning and downscaling for high-resolution spatiotemporal estimations of wind speed. Remote Sensing 11, 11 (2019), 1378.

[480] Songnian Li, Suzana Dragicevic, Francesc Antón Castro, Monika Sester, Stephan Winter, Arzu Coltekin, Christopher Pettit, Bin Jiang, James Haworth, Alfred Stein, and Tao Cheng. 2016. Geospatial big data handling theory and methods: A review and research challenges. ISPRS fournal of Photogrammetry and Remote Sensing 115 (2016), 119-133.

[481] Wan Li, Li Ni, Zhao-liang Li, Si-Bo Duan, and Hua Wu. 2019. Evaluation of machine learning algorithms in spatial downscaling of MODIS land surface temperature. IEEE fournal of Selected Topics in Applied Earth Observations and Remote Sensing 12, 7 (2019), 2299-2307.

[482] Konstantinos Liakos, Patrizia Busato, Dimitrios Moshou, Simon Pearson, and Dionysis Bochtis. 2018. Machine learning in agriculture: A review. Sensors 18, 8 (2018), 2674.

[483] Jianming Lian, Y. Sun, Karanjit Kalsi, Steven E. Widergren, Di Wu, and Huiying Ren. 2018. Transactive System: Part II: Analysis of Two Pilot Transactive Systems using Foundational Theory and Metrics. Technical Report. Pacific Northwest National Lab, Richland, WA.

[484] Jianming Lian, Wei Zhang, Y. Sun, Laurentiu D. Marinovici, Karanjit Kalsi, and Steven E. Widergren. 2018. Transactive System: Part I: Theoretical Underpinnings of Payoff Functions, Control Decisions, Information Privacy, and Solution Concepts. Technical Report. Pacific Northwest National Lab, Richland, WA.

[485] Albert C. Lin. 2013. Does geoengineering present a moral hazard. Ecology Law Quarterly 40, 3 (2013), 673.

[486] J. Ling and J. Templeton. 2015. Evaluation of machine learning algorithms for prediction of regions of high Reynolds averaged Navier Stokes uncertainty. Physics of Fluids 27, 085103 (2015).

[487] Christopher D. Lippitt, John Rogan, Zhe Li, J. Ronald Eastman, and Trevor G. Jones. 2008. Mapping selective logging in mixed deciduous forest. Photogrammetric Engineering \& Remote Sensing 74, 10 (2008), 1201-1211.

[488] Yunjie Liu, Evan Racah, Prabhat, Joaquin Correa, Amir Khosrowshahi, David Lavers, Kenneth Kunkel, Michael Wehner, and William Collins. 2016. Application of deep convolutional neural networks for detecting extreme weather in climate datasets. In International Conference on Advances in Big Data Analytics.

[489] Yi Liu, Chao Yang, Li Jiang, Shengli Xie, and Yan Zhang. 2019. Intelligent edge computing for IoT-Based energy management in smart cities. IEEE Network 33, 2 (2019), 111-117.

[490] Yue Liu, Tianlu Zhao, Wangwei Ju, and Siqi Shi. 2017. Materials discovery and design using machine learning. fournal of Materiomics 3, 3 (2017), 159-177.

[491] Alexey Lokhov. 2011. Technical and economic aspects of load following with nuclear power plants. NEA, OECD, Paris, France.

[492] Vincenzo Lomonaco, Angelo Trotta, Marta Ziosi, Juan De Dios Yáñez Ávila, and Natalia Díaz-Rodríguez. 2018. Intelligent drone swarm for search and rescue operations at sea. Preprint arXiv:1811.05291 (2018).

[493] Tania B. Lopez-Garcia, Alberto Coronado-Mendoza, and José A. Domínguez-Navarro. 2020. Artificial neural networks in microgrids: A review. Engineering Applications of Artificial Intelligence 95 (2020), 103894.

[494] R. Louf and M. Barthelemy. 2014. A typology of street patterns. Fournal of The Royal Society Interface 11, 101 (2014), 20140924-20140924.

[495] Miaojia Lu, Morteza Taiebat, Ming Xu, and Shu-Chien Hsu. 2018. Multiagent spatial simulation of autonomous taxis for urban commute: Travel economics and environmental impacts. Journal of Urban Planning and Development 144, 4 (2018), 04018033.

[496] Zhenyu Lu, Jungho Im, Jinyoung Rhee, and Michael Hodgson. 2014. Building type classification using spatial and landscape attributes derived from LiDAR remote sensing data. Landscape and Urban Planning 130 (2014), 134-148. 
[497] D. D. Lucas, R. Klein, J. Tannahill, D. Ivanova, S. Brandon, D. Domyancic, and Y. Zhang. 2013. Failure analysis of parameter-induced simulation crashes in climate models. Geoscientific Model Development 6, 4 (2013), 1157-1171.

[498] Alexandra Luccioni, Emily Baylor, and Nicolas Duchene. 2020. Analyzing sustainability reports using natural language processing. arXiv preprint arXiv:2011.08073 (2020).

[499] O. Lucon, D. Ürge Vorsatz, A. Zain Ahmed, P. Bertoldi, L. F. Cabeza, N. Eyre, A. Gadgil, L. D. D. Harvey, Y. Jiang, S Liphoto, S. Mirasgedis, S. Murakami, J. Parikh, C. Pyke, and M. V. Vilariño. 2014. Buildings. In Climate Change 2014: Mitigation of Climate Change. Contribution of Working Group III to the Fifth Assessment Report of the Intergovernmental Panel on Climate Change. Edenhofer, O., R. Pichs-Madruga, Y. Sokona, E. Farahani, S. Kadner, K. Seyboth, A. Adler, I. Baum, S. Brunner, P. Eickemeier, B. Kriemann, J. Savolainen, S. Schlömer, C. von Stechow, T. Zwickel, and J. C. Minx (Eds.). Cambridge University Press, Cambridge, UK.

[500] Jie Luo, Yongming Zhang, Feng Wang, Jinjun Wang, and Qixing Zhang. 2018. Applying machine learning to estimate the optical properties of black carbon fractal aggregates. Fournal of Quantitative Spectroscopy and Radiative Transfer 215 (2018), 1-8.

[501] Björn Lütjens, Lucas Liebenwein, and Katharina Kramer. 2019. Machine learning-based estimation of forest carbon stocks to increase transparency of forest preservation efforts. arXiv preprint arXiv:1912.07850 (2019).

[502] Andreas Lydakis, Jenica M. Allen, Marek Petrik, and Tim Szewczyk. 2018. Computing robust strategies for managing invasive plants. Retrieved from https://marek.petrik.us/pub/Lydakis2018.pdf.

[503] Wei Ma, Kendall Nowocin, Niraj Marathe, and George H. Chen. 2019. An interpretable produce price forecasting system for small and marginal farmers in India using collaborative filtering and adaptive nearest neighbors. In 10th International Conference on Information and Communication Technologies and Development. ACM, 6.

[504] Wei Ma and Zhen (Sean) Qian. 2018. Estimating multi-year 24/7 origin-destination demand using high-granular multi-source traffic data. Transportation Research Part C: Emerging Technologies 96 (2018), 96-121.

[505] MAAP. 2020. Monitoring of the Andean Amazon Project. Retrieved from https://maaproject.org/about-maap/.

[506] K. MacDicken, Ö. Jonsson, L. Piña, S. Maulo, V. Contessa, Y. Adikari, M. Garzuglia, E. Lindquist, G. Reams, and R. D’Annunzio. 2016. Global Forest Resources Assessment 2015: How Are the World's Forests Changing? FAO.

[507] David MacKay. 2008. Sustainable Energy-Without the Hot Air. UIT Cambridge.

[508] Douglas G. MacMartin and Ben Kravitz. 2019. The engineering of climate engineering. Annual Review of Control, Robotics, and Autonomous Systems 2 (2019), 445-467.

[509] Douglas G. MacMartin, Ben Kravitz, and Philip J. Rasch. 2015. On solar geoengineering and climate uncertainty. Geophysical Research Letters 42, 17 (2015), 7156-7161.

[510] Roberto Maestre, Juan Ramón Duque, Alberto Rubio, and Juan Arévalo. 2018. Reinforcement learning for fair dynamic pricing. In Intelligent Systems and Applications. IntelliSys 2018. Advances in Intelligent Systems and Computing, K. Arai, S. Kapoor, R. Bhatia (Eds). vol. 868, Springer, Cham, 120-135.

[511] Natalie M. Mahowald, Daniel S. Ward, Scott C. Doney, Peter G. Hess, and James T. Randerson. 2017. Are the impacts of land use on warming underestimated in climate policy? Environmental Research Letters 12, 9 (2017), 094016.

[512] Jorge Maldonado-Correa, J. C. Solano, and Marco Rojas-Moncayo. 2019. Wind power forecasting: A systematic literature review. Wind Engineering 45, 2 (2019), 413-426.

[513] Kolya Malkin, Caleb Robinson, Le Hou, Rachel Soobitsky, Jacob Czawlytko, Dimitris Samaras, Joel Saltz, Lucas Joppa, and Nebojsa Jojic. 2018. Label super-resolution networks. In ICLR 2019 Conference.

[514] Jordan M. Malof, Kyle Bradbury, Leslie M. Collins, and Richard G. Newell. 2016. Automatic detection of solar photovoltaic arrays in high resolution aerial imagery. Applied Energy 183 (2016), 229-240.

[515] Ed Manley, Chen Zhong, and Michael Batty. 2018. Spatiotemporal variation in travel regularity through transit user profiling. Transportation 45, 3 (2018), 703-732.

[516] Vukosi Marivate and Nyalleng Moorosi. 2017. Employment relations: A data driven analysis of job markets using online job boards and online professional networks. In International Conference on Web Intelligence. ACM, 1110-1113.

[517] Mason Marks. 2019. Robots in space: Sharing our world with autonomous delivery vehicles. SSRN Electronic fournal (2019).

[518] Jeffrey Marlow, Chiara Borrelli, Sean P. Jungbluth, Colleen Hoffman, Jennifer Marlow, and Peter R. Girguis. 2017. Opinion: Telepresence is a potentially transformative tool for field science. Proceedings of the National Academy of Sciences 114, 19 (2017), 4841-4844.

[519] Antoine Marot, Benjamin Donnot, Gabriel Dulac-Arnold, Adrian Kelly, Aïdan O’Sullivan, Jan Viebahn, Mariette Awad, Isabelle Guyon, Patrick Panciatici, and Camilo Romero. 2021. Learning to run a power network challenge: A retrospective analysis. In Proceedings of the Machine Learning Research Competition and Demonstration Track (NeurIPS'20). 133: 112-132.

[520] Antoine Marot, Benjamin Donnot, Camilo Romero, Balthazar Donon, Marvin Lerousseau, Luca Veyrin-Forrer, and Isabelle Guyon. 2020. Learning to run a power network challenge for training topology controllers. Electric Power Systems Research 189 (2020), 106635. 
[521] Jochem Marotzke, Christian Jakob, Sandrine Bony, Paul A. Dirmeyer, Paul A. O'Gorman, Ed Hawkins, Sarah PerkinsKirkpatrick, Corinne Le Quéré, Sophie Nowicki, Katsia Paulavets, Sonia I. Seneviratne, Bjorn Stevens, and Matthias Tuma. 2017. Climate research must sharpen its view. Nature Climate Change 7, 2 (2017), 89.

[522] David Martimort and Wilfried Sand-Zantman. 2016. A mechanism design approach to climate agreements. fournal of the European Economic Association 14, 3 (2016), 669-718

[523] Carlo Brancucci Martinez-Anido, Benjamin Botor, Anthony R. Florita, Caroline Draxl, Siyuan Lu, Hendrik F. Hamann, and Bri-Mathias Hodge. 2016. The value of day-ahead solar power forecasting improvement. Solar Energy 129 (2016), 192-203.

[524] Johan Mathe, Nina Miolane, Nicolas Sebastien, and Jeremie Lequeux. 2019. PVNet: A LRCN architecture for spatiotemporal photovoltaic powerforecasting from numerical weather prediction. Preprint arXiv:1902.01453 (2019).

[525] Alessandro Mattiussi, Michele Rosano, and Patrizia Simeoni. 2014. A decision support system for sustainable energy supply combining multi-objective and multi-attribute analysis: An Australian case study. Decision Support Systems 57 (2014), 150-159.

[526] Ehsan Mazloumi, Geoff Rose, Graham Currie, and Sara Moridpour. 2011. Prediction intervals to account for uncertainties in neural network predictions: Methodology and application in bus travel time prediction. Engineering Applications of Artificial Intelligence 24, 3 (2011), 534-542.

[527] Justin McClellan, David W. Keith, and Jay Apt. 2012. Cost analysis of stratospheric albedo modification delivery systems. Environmental Research Letters 7, 3 (2012), 034019.

[528] Nate G. McDowell, Nicholas C. Coops, Pieter S. A. Beck, Jeffrey Q. Chambers, Chandana Gangodagamage, Jeffrey A. Hicke, Cho-ying Huang, Robert Kennedy, Dan J. Krofcheck, Marcy Litvak, Arjan J. H. Meddens, Jordan Muss, Robinson Negrón-Juarez, Changhui Peng, Amanda M. Schwantes, Jennifer J. Swenson, Louis J. Vernon, A. Park Williams, Chonggang Xu, Maosheng Zhao, Steve W. Running, and Craig D. Allen. 2015. Global satellite monitoring of climate-induced vegetation disturbances. Trends in Plant Science 20, 2 (2015), 114-123.

[529] Amy McGovern, Kimberly L. Elmore, David John Gagne, Sue Ellen Haupt, Christopher D. Karstens, Ryan Lagerquist, Travis Smith, and John K. Williams. 2017. Using artificial intelligence to improve real-time decision-making for highimpact weather. Bulletin of the American Meteorological Society 98, 10 (2017), 2073-2090.

[530] Scott Mcquade and Claire Monteleoni. 2012. Global climate model tracking using geospatial neighborhoods. 26th AAAI Conference on Artificial Intelligence.

[531] Patrick Meier. 2013. Human computation for disaster response. In Handbook of Human Computation. Springer, 95-104.

[532] Antonella Meneghetti and Luca Monti. 2015. Greening the food supply chain: An optimisation model for sustainable design of refrigerated automated warehouses. International fournal of Production Research 53, 21 (2015), 6567-6587.

[533] Sreejith Menon, Tanya Berger-Wolf, Emre Kiciman, Lucas Joppa, Charles V. Stewart, Jason Parham, Jonathan Crall, Jason Holmberg, and Jonathan Van Oast. 2016. Animal population estimation using Flickr images. In 2nd International Workshop on the Social Web for Environmental and Ecological Monitoring.

[534] MethaneSAT. 2021. MethaneSAT. Retrieved from https://www.methanesat.org/.

[535] Microsoft. 2018. Computer generated building footprints for the United States. Retrieved from https://github.com/ Microsoft/USBuildingFootprints.

[536] Nikola Milojevic-Dupont and Felix Creutzig. 2020. Machine learning for geographically differentiated climate change mitigation in urban areas. Sustainable Cities and Society 64 (2020), 102526.

[537] Nikola Milojevic-Dupont, Nicolai Hans, Lynn H. Kaack, Marius Zumwald, François Andrieux, Daniel de Barros Soares, Steffen Lohrey, Peter-Paul Pichler, and Felix Creutzig. 2020. Learning from urban form to predict building heights. PLOS One 15, 12 (2020), 1-22.

[538] Budiman Minasny, Budi Indra Setiawan, Satyanto Krido Saptomo, and Alex B. McBratney. 2018. Open digital mapping as a cost-effective method for mapping peat thickness and assessing the carbon stock of tropical peatlands. Geoderma 313 (2018), 25-40.

[539] Riccardo Minciardi, Massimo Paolucci, Michela Robba, and Roberto Sacile. 2008. Multi-objective optimization of solid waste flows: Environmentally sustainable strategies for municipalities. Waste Management 28, 11 (2008), 2202-2212

[540] Jan C. Minx, William F. Lamb, Max W. Callaghan, Sabine Fuss, Jerome Hilaire, Felix Creutzig, Thorben Amann, Tim Beringer, Wagner de Oliveira Garcia, Jens Hartmann, Tarun Khanna, Dominic Lenzi, Gunnar Luderer, Gregory F. Nemet, Joeri Rogelj, Pete Smith, Jose Luis Vicente Vicente, Jennifer Wilcox, and Maria del Mar Zamora Dominguez. 2018. Negative emissions-Part 1: Research landscape and synthesis. Environmental Research Letters 13, 6 (2018), 063001.

[541] Sidhant Misra, Line Roald, and Yeesian Ng. 2018. Learning for constrained optimization: Identifying optimal active constraint sets. arXiv preprint arXiv:1802.09639 (2018).

[542] Shaoxing Mo, Yinhao Zhu, Nicholas Zabaras, Xiaoqing Shi, and Jichun Wu. 2019. Deep convolutional encoderdecoder networks for uncertainty quantification of dynamic multiphase flow in heterogeneous media. Water Resources Research 55, 1 (2019), 703-728. 
[543] Elena Mocanu, Decebal Constantin Mocanu, Phuong H. Nguyen, Antonio Liotta, Michael E. Webber, Madeleine Gibescu, and Johannes G. Slootweg. 2019. On-line building energy optimization using deep reinforcement learning. IEEE Transactions on Smart Grid 10, 4 (2019), 3698-3708.

[544] Elena Mocanu, Phuong H. Nguyen, Wil L. Kling, and Madeleine Gibescu. 2016. Unsupervised energy prediction in a smart grid context using reinforcement cross-building transfer learning. Energy and Buildings 116 (2016), 646-655.

[545] Signe Moe, Anne Marthine Rustad, and Kristian G. Hanssen. 2018. Machine learning in control systems: An overview of the state of the art. In International Conference on Innovative Techniques and Applications of Artificial Intelligence. Springer, 250-265.

[546] Nicholas Moehle, Enzo Busseti, Stephen Boyd, and Matt Wytock. 2019. Dynamic energy management. In Large Scale Optimization in Supply Chains and Smart Manufacturing. Springer, 69-126.

[547] Saeed Monajem and Farzan Ekram Nosratian. 2015. The evaluation of the spatial integration of station areas via the node place model; an application to subway station areas in Tehran. Transportation Research Part D: Transport and Environment 40 (2015), 14-27.

[548] C. Monteleoni, G. A. Schmidt, F. Alexander, A. Niculescu-Mizil, K. Steinhaeuser, M. Tippett, A. Banerjee, M. B. Blumenthal, A. R. Ganguly, J. E. Smerdon, and M. Tedesco. 2013. Climate jnformatic. In Computational Intelligent Data Analysis for Sustainable Development; Data Mining and Knowledge Discovery Series. T. Yu, N. Chawla, and S. Simoff (Eds.). CRC Press, Taylor \& Francis Group, Chapter 4, 81-126.

[549] Claire Monteleoni, Gavin A. Schmidt, Shailesh Saroha, and Eva Asplund. 2011. Tracking climate models. Statistical Analysis and Data Mining 4 (2011), 372-392.

[550] Claire A. Montgomery. 2014. Fire: An agent and a consequence of land use change. In The Oxford Handbook of Land Economics. OUP, 281.

[551] Joseph H. Montoya, Charlie Tsai, Aleksandra Vojvodic, and Jens K. Nørskov. 2015. The challenge of electrochemical ammonia synthesis: A new perspective on the role of nitrogen scaling relations. ChemSusChem 8, 13 (2015), 21802186.

[552] Aditi Moorthy, Robert De Kleine, Gregory Keoleian, Jeremy Good, and Geoff Lewis. 2017. Shared autonomous vehicles as a sustainable solution to the last mile problem: A case study of Ann Arbor-Detroit area. SAE International Journal of Passenger Cars-Electronic and Electrical Systems 10, 2 (2017), 328-336.

[553] Camilo Mora, Chelsie W. W. Counsell, Coral R. Bielecki, and Leo V. Louis. 2017. Twenty-seven ways a heat wave can kill you: Deadly heat in the era of climate change. Circulation: Cardiovascular Quality and Outcomes 10, 11 (2017), e004233.

[554] Camilo Mora, Bénédicte Dousset, Iain R. Caldwell, Farrah E. Powell, Rollan C. Geronimo, Coral R. Bielecki, Chelsie W. W. Counsell, Bonnie S. Dietrich, Emily T. Johnston, Leo V. Louis, Matthew P. Lucas, Marie M. McKenzie, Alessandra G. Shea, Han Tseng, Thomas W. Giambelluca, Lisa R. Leon, Ed Hawkins, and Clay Trauernicht. 2017. Global risk of deadly heat. Nature Climate Change 7, 7 (2017), 501.

[555] M. Granger Morgan. 2017. Theory and Practice in Policy Analysis: Including Applications in Science and Technology. Cambridge University Press.

[556] Shunsuke Mori, Toyoaki Washida, Atsushi Kurosawa, and Toshihiko Masui. 2018. Assessment of mitigation strategies as tools for risk management under future uncertainties: A multi-model approach. Sustainability Science 13, 2 (2018), 329-349.

[557] Dylan Moriarty, Laura Dobeck, and Sally Benson. 2014. Rapid surface detection of CO2 leaks from geologic sequestration sites. Energy Procedia 63 (2014), 3975-3983.

[558] Farnaz Mosannenzadeh, Maria Rosaria Di Nucci, and Daniele Vettorato. 2017. Identifying and prioritizing barriers to implementation of smart energy city projects in Europe: An empirical approach. Energy Policy 105 (2017), 191-201.

[559] Amir Mosavi, Mohsen Salimi, Sina Faizollahzadeh Ardabili, Timon Rabczuk, Shahaboddin Shamshirband, and Annamaria R. Varkonyi-Koczy. 2019. State of the art of machine learning models in energy systems, a systematic review. Energies 12, 7 (2019), 1301.

[560] José Antonio Moscoso-López, Ignacio Turias, Maria Jesús Jiménez-Come, Juan Jesús Ruiz-Aguilar, and María del Mar Cerbán. 2019. A two-stage forecasting approach for short-term intermodal freight prediction. International Transactions in Operational Research 26, 2 (2019), 642-666.

[561] Richard H. Moss, Jae A. Edmonds, Kathy A. Hibbard, Martin R. Manning, Steven K. Rose, Detlef P. van Vuuren, Timothy R. Carter, Seita Emori, Mikiko Kainuma, Tom Kram, Gerald A. Meehl, John F. B. Mitchell, Nebojsa Nakicenovic, Keywan Riahi, Steven J. Smith, Ronald J. Stouffer, Allison M. Thomson, John P. Weyant, and Thomas J. Wilbanks. 2010. The next generation of scenarios for climate change research and assessment. Nature 463, 7282 (2010), 747-756.

[562] Alberto Mucci. 2016. The Supermarket of the Future Knows Exactly What You're Eating. Retrieved from https:// www.vice.com/en_us/article/4xbppn/the-supermarket-of-the-future-knows-exactly-what-youre-eating.

[563] Khan Muhammad, Jaime Lloret, and Sung Wook Baik. 2019. Intelligent and energy-efficient data prioritization in green smart cities: Current challenges and future directions. IEEE Communications Magazine 57, 2 (2019), 60-65. 
[564] Fitore Muharemi, Doina Logofătu, and Florin Leon. 2019. Machine learning approaches for anomaly detection of water quality on a real-world data set. Journal of Information and Telecommunication 3, 3 (2019), 294-307.

[565] Surya Karthik Mukkavilli. 2019. EnviroNet: ImageNet for environment. In 18th Conference on Artificial and Computational Intelligence and its Applications to the Environmental Sciences. American Meteorological Society.

[566] T. Nathan Mundhenk, Goran Konjevod, Wesam A. Sakla, and Kofi Boakye. 2016. A large contextual dataset for classification, detection and counting of cars with deep learning. In European Conference on Computer Vision. Springer, 785-800.

[567] A. Murari, G. Vagliasindi, P. Arena, L. Fortuna, O. Barana, M. Johnson, and JET-EFDA Contributors. 2008. Prototype of an adaptive disruption predictor for JET based on fuzzy logic and regression trees. Nuclear Fusion 48, 3 (2008), 035010.

[568] Ernest Mwebaze, Washington Okori, and John Alexander Quinn. 2010. Causal structure learning for famine prediction. In 2010 AAAI Spring Symposium Series.

[569] Prashant Nagapurkar and Joseph D. Smith. 2019. Techno-economic optimization and social costs assessment of microgrid-conventional grid integration using genetic algorithm and artificial neural networks: A case study for two US cities. Journal of Cleaner Production 229 (2019), 552-569.

[570] Harini Nagendra, Xuemei Bai, Eduardo S. Brondizio, and Shuaib Lwasa. 2018. The urban south and the predicament of global sustainability. Nature Sustainability 1, 7 (2018), 341.

[571] Daisik Nam, Hyunmyung Kim, Jaewoo Cho, and R. Jayakrishnan. 2017. A model based on deep learning for predicting travel mode choice. In 96th Annual Meeting of the Transportation Research Board, Washington, DC. 8-12.

[572] Vishnuteja Nanduri and Tapas Das. 2007. A reinforcement learning model to assess market power under auctionbased energy pricing. IEEE Transactions on Power Systems 22, 1 (2007), 85-95.

[573] NASA Science. 2019. The study of Earth as an integrated system. Retrieved from https://climate.nasa.gov/nasa science/science/.

[574] Roshanak Nateghi. 2018. Multi-dimensional infrastructure resilience modeling: An application to hurricane-prone electric power distribution systems. IEEE Access 6 (2018), 13478-13489.

[575] National Academies of Sciences, Engineering, and Medicine. 2019. Negative Emissions Technologies and Reliable Sequestration: A Research Agenda. The National Academies Press, Washington, DC.

[576] National Grid ESO. 2019. Carbon Intensity API. Retrieved from https://carbonintensity.org.uk/.

[577] Nature Physics. 2016. Insight: Nuclear Fusion. Retrieved from https://www.nature.com/collections/bccqhmkbyw.

[578] NCX. 2021. NCX. Retrieved from https://www.ncx.com.

[579] Paolo Neirotti, Alberto De Marco, Anna Corinna Cagliano, Giulio Mangano, and Francesco Scorrano. 2014. Current trends in smart city initiatives: Some stylised facts. Cities 38 (2014), 25-36.

[580] Gregory F. Nemet, Max W. Callaghan, Felix Creutzig, Sabine Fuss, Jens Hartmann, Jérôme Hilaire, William F. Lamb, Jan C. Minx, Sophia Rogers, and Pete Smith. 2018. Negative emissions-Part 3: Innovation and upscaling. Environmental Research Letters 13, 6 (2018), 063003.

[581] Van Nhan Nguyen, Robert Jenssen, and Davide Roverso. 2018. Automatic autonomous vision-based power line inspection: A review of current status and the potential role of deep learning. International fournal of Electrical Power \& Energy Systems 99 (2018), 107-120.

[582] Roger Nkambou, Riichiro Mizoguchi, and Jacqueline Bourdeau. 2010. Advances in Intelligent Tutoring Systems. Vol. 308. Springer Science \& Business Media.

[583] Silvio Nocera, Cayetano Ruiz-Alarcón-Quintero, and Federico Cavallaro. 2018. Assessing carbon emissions from road transport through traffic flow estimators. Transportation Research Part C: Emerging Technologies 95 (2018), 125-148.

[584] Mehdi Noori and Omer Tatari. 2016. Development of an agent-based model for regional market penetration projections of electric vehicles in the United States. Energy 96 (2016), 215-230.

[585] Mohammad Sadegh Norouzzadeh, Anh Nguyen, Margaret Kosmala, Alexandra Swanson, Meredith S. Palmer, Craig Packer, and Jeff Clune. 2018. Automatically identifying, counting, and describing wild animals in camera-trap images with deep learning. Proceedings of the National Academy of Sciences 115, 25 (2018), E5716-E5725.

[586] Peyman Noursalehi, Haris N. Koutsopoulos, and Jinhua Zhao. 2018. Real time transit demand prediction capturing station interactions and impact of special events. Transportation Research Part C: Emerging Technologies 97 (2018), 277-300.

[587] Peer Nowack, Peter Braesicke, Joanna Haigh, Nathan Luke Abraham, and John Pyle. 2018. Using machine learning to build temperature-based ozone parameterizations for climate sensitivity simulations. Environmental Research Letters 13, 10 (2018), 104016.

[588] Sudhakar V. Nuti, Brian Wayda, Isuru Ranasinghe, Sisi Wang, Rachel P. Dreyer, Serene I. Chen, and Karthik Murugiah. 2014. The use of Google Trends in health care research: A systematic review. PloS One 9, 10 (2014), e109583.

[589] Alex Nutkiewicz, Zheng Yang, and Rishee K. Jain. 2018. Data-driven urban energy simulation (DUE-S): A framework for integrating engineering simulation and machine learning methods in a multi-scale urban energy modeling workflow. Applied Energy 225 (2018), 1176-1189. 
[590] Benjamin D. Nye. 2015. Intelligent tutoring systems by and for the developing world: A review of trends and approaches for educational technology in a global context. International fournal of Artificial Intelligence in Education 25, 2 (2015), 177-203.

[591] Stanford Graduate School of Business. 2017. Andrew Ng: Artificial Intelligence is the New Electricity. Retrieved from https://www.youtube.com/watch?v=21EiKfQYZXc.

[592] Union of Concerned Scientists. 2019. UCS Position on Solar Geoengineering. Retrieved from https://www.ucsusa. $\mathrm{org} / \mathrm{sites} / \mathrm{default} /$ files/attach/2019/gw-position-Solar-Geoengineering-022019.pdf.

[593] U.S. Office of Energy Efficiency \& Renewable Energy. 2019. Energy Department Awards \$5.5 Million to Apply Machine Learning to Geothermal Exploration. Retrieved from https://www.energy.gov/eere/articles/energydepartment-awards-55-million-apply-machine-learning-geothermal-exploration.

[594] Timothy Oladunni and Sharad Sharma. 2016. Hedonic housing theory-a machine learning investigation. In 2016 15th IEEE International Conference on Machine Learning and Applications.

[595] Mark Olsthoorn, Joachim Schleich, and Corinne Faure. 2019. Exploring the diffusion of low-energy houses: An empirical study in the European Union. Energy Policy 129 (2019), 1382-1393.

[596] Alexandra Olteanu, Carlos Castillo, Fernando Diaz, and Sarah Vieweg. 2014. CrisisLex: A lexicon for collecting and filtering microblogged communications in crises. In 8th International AAAI Conference on Weblogs and Social Media.

[597] Hichem Omrani. 2015. Predicting travel mode of individuals by machine learning. Transportation Research Procedia 10 (2015), 840-849.

[598] Charles C. Onu, Innocent Udeogu, Eyenimi Ndiomu, Urbain Kengni, Doina Precup, Guilherme M. Sant'Anna, Edward Alikor, and Peace Opara. 2017. Ubenwa: Cry-based diagnosis of birth asphyxia. Preprint arXiv:1711.06405 (2017).

[599] Tara O'Shea. 2019. Developing the World's First Indicator of Forest Carbon Stocks \& Emissions. Retrieved from https://www.planet.com/pulse/developing-the-worlds-first-indicator-of-forest-carbon-stocks-emissions/.

[600] Barak Oshri, Annie Hu, Peter Adelson, Xiao Chen, Pascaline Dupas, Jeremy Weinstein, Marshall Burke, David Lobell, and Stefano Ermon. 2018. Infrastructure quality assessment in Africa using satellite imagery and deep learning. In 24th ACM SIGKDD International Conference on Knowledge Discovery \& Data Mining. ACM, 616-625.

[601] Fred Otieno, Nathan Williams, and Patrick McSharry. 2018. Forecasting energy demand for microgrids over multiple horizons. In 2018 IEEE PES/IAS PowerAfrica. IEEE, 457-462.

[602] Edward O’Dwyer, Indranil Pan, Salvador Acha, and Nilay Shah. 2019. Smart energy systems for sustainable smart cities: Current developments, trends and future directions. Applied Energy 237 (2019), 581-597.

[603] Pachama. 2021. Pachama. Retrieved from https://pachama.com/.

[604] Rajendra K. Pachauri. 2014. Climate Change 2014 Synthesis Report. IPCC.

[605] Michela Paganini, Luke de Oliveira, and Benjamin Nachman. 2018. Accelerating science with generative adversarial networks: An application to 3D particle showers in multilayer calorimeters. Physical Review Letters 120, 4 (2018), 042003.

[606] Susan E. Page, Florian Siegert, John O. Rieley, Hans-Dieter V. Boehm, Adi Jaya, and Suwido Limin. 2002. The amount of carbon released from peat and forest fires in Indonesia during 1997. Nature 420, 6911 (2002), 61.

[607] Liviu Panait and Sean Luke. 2005. Cooperative multi-agent learning: The state of the art. Autonomous Agents and Multi-Agent Systems 11, 3 (2005), 387-434.

[608] K. S. Pandya and S. K. Joshi. 2008. A survey of optimal power flow methods. fournal of Theoretical \& Applied Information Technology 4, 5 (2008), 450-458.

[609] Mathaios Panteli and Pierluigi Mancarella. 2015. The grid: Stronger bigger smarter?: Presenting a conceptual framework of power system resilience. IEEE Power Energy Mag 13, 3 (2015), 58-66.

[610] Sokratis Papadopoulos, Bartosz Bonczak, and Constantine E. Kontokosta. 2018. Pattern recognition in building energy performance over time using energy benchmarking data. Applied Energy 221 (2018), 576-586.

[611] Sokratis Papadopoulos and Constantine E. Kontokosta. 2019. Grading buildings on energy performance using city benchmarking data. Applied Energy 233-234 (2019), 244-253.

[612] Faizal Parish, A. A. Sirin, D. Charman, Hans Joosten, T. Yu Minaeva, and Marcel Silvius. 2008. Assessment on peatlands, biodiversity and climate change: Main report. Global Environment Centre, Kuala Lumpur and Wetlands International, Wageningen.

[613] Byeonghwa Park and Jae Bae. 2015. Using machine learning algorithms for housing price prediction: The case of Fairfax County, Virginia housing data. Expert Systems with Applications 42 (2015), 2928-2934.

[614] June Young Park, Thomas Dougherty, Hagen Fritz, and Zoltan Nagy. 2019. LightLearn: An adaptive and occupant centered controller for lighting based on reinforcement learning. Building and Environment 147 (2019), 397-414.

[615] Andy Parker and Peter J. Irvine. 2018. The risk of termination shock from solar geoengineering. Earth's Future 6, 3 (2018), 456-467.

[616] David Pastor-Escuredo, Alfredo Morales-Guzmán, Yolanda Torres-Fernández, Jean-Martin Bauer, Amit Wadhwa, Carlos Castro-Correa, Liudmyla Romanoff, Jong Gun Lee, Alex Rutherford, Vanessa Frias-Martinez, Nuria Oliver, 
Enrique Frias-Martinez, and Miguel Luengo-Oroz. 2014. Flooding through the lens of mobile phone activity. In IEEE Global Humanitarian Technology Conference (GHTC'14). IEEE, 279-286.

[617] Nikolaos G. Paterakis, Elena Mocanu, Madeleine Gibescu, Bart Stappers, and Walter van Alst. 2017. Deep learning versus traditional machine learning methods for aggregated energy demand prediction. In 2017 IEEE PES Innovative Smart Grid Technologies Conference Europe (ISGT-Europe'17). IEEE, 1-6.

[618] C. V. Patton, D. S. Sawicki, and J. Clark. 2015. Basic Methods of Policy Analysis and Planning. Taylor \& Francis

[619] Judea Pearl. 2019. The seven tools of causal inference, with reflections on machine learning. Communications of the ACM 62, 3 (2019), 54-60.

[620] A. Pee, D. Pinner, O. Roelofsen, K. Somers, E. Speelman, and M. Witteveen. 2018. Decarbonization of industrial sectors: The next frontier. Retrieved from https://www.mckinsey.com/industries/oil-and-gas/our-insights/decarbonizationof-industrial-sectors-the-next-frontier.

[621] Mark Pelling. 2010. Adaptation to Climate Change: From Resilience to Transformation. Routledge.

[622] Alex Pentland, David Lazer, Devon Brewer, and Tracy Heibeck. 2009. Using reality mining to improve public health and medicine. Studies in Health Technology and Informatics 149 (2009), 93-102.

[623] Kasun S. Perera, Zeyar Aung, and Wei Lee Woon. 2014. Machine learning techniques for supporting renewable energy generation and integration: A survey. In International Workshop on Data Analytics for Renewable Energy Integration. Springer, 81-96.

[624] Gregorij V. Pereverzev and P. N. Yushmanov. 2002. ASTRA. Automated System for TRansport Analysis in a tokamak. Aspen Technology Inc., San Diego, CA.

[625] M. C. Perignon, P. Passalacqua, T. M. Jarriel, J. M. Adams, and I. Overeem. 2018. Patterns of geomorphic processes across deltas using image analysis and machine learning. In AGU Fall Meeting Abstracts.

[626] Michael Pertl, Kai Heussen, Oliver Gehrke, and Michel Rezkalla. 2016. Voltage estimation in active distribution grids using neural networks. In 2016 IEEE Power and Energy Society General Meeting (PESGM'16). IEEE, 1-5.

[627] Fahad Pervaiz, Mansoor Pervaiz, Nabeel Abdur Rehman, and Umar Saif. 2012. FluBreaks: Early epidemic detection from Google flu trends. Journal of Medical Internet Research 14, 5 (2012), e125.

[628] Katherine Hoffmann Pham, Jeremy Boy, and Miguel Luengo-Oroz. 2018. Data fusion to describe and quantify search and rescue operations in the Mediterranean sea. In 2018 IEEE 5th International Conference on Data Science and Advanced Analytics (DSAA'18). IEEE, 514-523.

[629] Paulo Picchetti. 2017. Hedonic residential property price estimation using geospatial data: A machine-learning approach. Instituto Brasileiro de Economia.

[630] Niels Pinkwart. 2016. Another 25 years of AIED? Challenges and opportunities for intelligent educational technologies of the future. International fournal of Artificial Intelligence in Education 26, 2 (2016), 771-783.

[631] Giuseppe Pinto, Marco Savino Piscitelli, José Ramón Vázquez-Canteli, Zoltán Nagy, and Alfonso Capozzoli. 2021. Coordinated energy management for a cluster of buildings through deep reinforcement learning. Energy 229 (2021), 120725.

[632] William A. Pizer. 2006. Choosing price or quantity controls for greenhouse gases. In The RFF Reader in Environmental and Resource Policy. Wallace E. Oates (Ed.). Resources for the Future, 225-234.

[633] Erica L. Plambeck. 2012. Reducing greenhouse gas emissions through operations and supply chain management. Energy Economics 34 (2012), S64-S74.

[634] PlantSnap. 2021. PlantSnap. Retrieved from https://www.plantsnap.com/.

[635] S. D. Pohekar and M. Ramachandran. 2004. Application of multi-criteria decision making to sustainable energy planning-A review. Renewable and Sustainable Energy Reviews 8, 4 (2004), 365-381.

[636] J. R. Porter, L. Xie, A. J. Challinor, K. Cochrane, M. M. Howden, D. B. Lobell, and M. I. Travasso. 2014. Food security and food production systems. In Climate Change 2014: Impacts, Adaptation, Vulnerability. IPCC, 485-533.

[637] Christopher Potter, Shyam Boriah, Michael Steinbach, Vipin Kumar, and Steven Klooster. 2008. Terrestrial vegetation dynamics and global climate controls. Climate Dynamics 31, 1 (2008), 67-78.

[638] PowerTAC. 2019. PowerTAC. Retrieved from https://powertac.org/.

[639] Gautam Prasad, Upendra Reddy Vuyyuru, and Mithun Das Gupta. 2019. Agriculture commodity arrival prediction using remote sensing data: insights and beyond. In KDD Feed Workshop 2019.

[640] Christopher J. Preston. 2013. Ethics and geoengineering: Reviewing the moral issues raised by solar radiation management and carbon dioxide removal. Wiley Interdisciplinary Reviews: Climate Change 4, 1 (2013), 23-37.

[641] Ariel D. Procaccia. 2013. Cake cutting: Not just child's play. Communications of the ACM 56, 7 (2013), $78-87$.

[642] Jonathan Proctor, Solomon Hsiang, Jennifer Burney, Marshall Burke, and Wolfram Schlenker. 2018. Estimating global agricultural effects of geoengineering using volcanic eruptions. Nature 560, 7719 (2018), 480.

[643] Project Zamba. 2019. Project Zamba Computer Vision for Wildlife Research \& Conservation. Retrieved from https: //zamba.drivendata.org/. 
[644] UN Global Pulse. 2013. Landscaping Study: Digital Signals \& Access to Finance in Kenya. Retrived from https://www. unglobalpulse.org/projects/Kenyan-access-finance.

[645] UN Global Pulse. 2015. Using mobile phone data and airtime credit purchases to estimate food security. New York: UN World Food Programme (WFP), Université Catholique de Louvain, Real Impact Analytics, Pulse Lab New York.

[646] UN Global Pulse. 2017. Improving Professional Training in Indonesia with Gaming Data. http://unglobalpulse.org/ sites/default/files/ProjectBrief-ImprovingProfressionalTraininginIndonesiawithGamingData.pdf.

[647] UN Global Pulse. 2017. Social Media and Forced Displacement: Big Data Analytics \& Machine Learning. Retrived from https://www.unhcr.org/innovation/wp-content/uploads/2017/09/FINAL-White-Paper.pdf.

[648] John Quinn, Vanessa Frias-Martinez, and Lakshminarayan Subramanian. 2014. Computational sustainability and artificial intelligence in the developing world. AI Magazine 35, 3 (2014), 36.

[649] John A. Quinn, Alfred Andama, Ian Munabi, and Fred N. Kiwanuka. 2014. Automated blood smear analysis for mobile malaria diagnosis. Mobile Point-of-Care Monitors and Diagnostic Device Design 31 (2014), 115.

[650] John A. Quinn, Marguerite M. Nyhan, Celia Navarro, Davide Coluccia, Lars Bromley, and Miguel Luengo-Oroz. 2018. Humanitarian applications of machine learning with remote-sensing data: Review and case study in refugee settlement mapping. Philosophical Transactions of the Royal Society A: Mathematical, Physical and Engineering Sciences 376, 2128 (2018), 20170363.

[651] Julianne D. Quinn, Patrick M. Reed, and Klaus Keller. 2017. Direct policy search for robust multi-objective management of deeply uncertain socio-ecological tipping points. Environmental Modelling \& Software 92 (2017), 125-141.

[652] Evan Racah, Christopher Beckham, Tegan Maharaj, Samira Ebrahimi Kahou, Prabhat, and Chris Pal. 2017. ExtremeWeather: A large-scale climate dataset for semi-supervised detection, localization, and understanding of extreme weather events. In Advances in Neural Information Processing Systems. 3402-3413.

[653] Paul Raccuglia, Katherine C. Elbert, Philip D. F. Adler, Casey Falk, Malia B. Wenny, Aurelio Mollo, Matthias Zeller, Sorelle A. Friedler, Joshua Schrier, and Alexander J. Norquist. 2016. Machine-learning-assisted materials discovery using failed experiments. Nature 533, 7601 (2016), 73.

[654] Rajkumar Ragupathi and Tapas Das. 2004. A stochastic game approach for modeling wholesale energy bidding in deregulated power markets. IEEE Transactions on Power Systems 19, 2 (2004), 849-856.

[655] Varun Rai, D. Cale Reeves, and Robert Margolis. 2016. Overcoming barriers and uncertainties in the adoption of residential solar PV. Renewable Energy 89 (2016), 498-505.

[656] Varun Rai and Scott A. Robinson. 2015. Agent-based modeling of energy technology adoption: Empirical integration of social, behavioral, economic, and environmental factors. Environmental Modelling \& Software 70 (2015), 163-177.

[657] Rainforest Connection. 2021. Rainforest Connection. Retrieved from https://rfcx.org.

[658] Maziar Raissi and George Em Karniadakis. 2018. Hidden physics models: Machine learning of nonlinear partial differential equations. Journal of Computational Physics 357 (2018), 125-141.

[659] Maziar Raissi, Paris Perdikaris, and George Em Karniadakis. 2017. Physics informed deep learning (Part I): Datadriven solutions of nonlinear partial differential equations. arXiv preprint arXiv:1711.10561 (2017).

[660] Eric Ralls. 2018. Systems and methods for electronically identifying plant species. US Patent App. 15/973660.

[661] Sarvapali Ramchurn, Perukrishnen Vytelingum, Alex Rogers, and Nicholas R. Jennings. 2012. Putting the "smarts" into the smart grid: A grand challenge for artificial intelligence. Communications of the ACM 55, 4 (2012), 86-97.

[662] Sarvapali D. Ramchurn, Perukrishnen Vytelingum, Alex Rogers, and Nick Jennings. 2011. Agent-based control for decentralised demand side management in the smart grid. In 10th International Conference on Autonomous Agents and Multiagent Systems-Volume 1. International Foundation for Autonomous Agents and Multiagent Systems, 5-12.

[663] Sarvapali D. Ramchurn, Perukrishnen Vytelingum, Alex Rogers, and Nicholas R. Jennings. 2011. Agent-based homeostatic control for green energy in the smart grid. ACM Transactions on Intelligent Systems and Technology 2, 4 (2011), 35.

[664] Pushpendra Rana and Daniel C. Miller. 2019. Machine learning to analyze the social-ecological impacts of natural resource policy: Insights from community forest management in the Indian Himalaya. Environmental Research Letters 14, 2 (2019), 024008.

[665] Marie-Ève Rancourt, Jean-François Cordeau, Gilbert Laporte, and Ben Watkins. 2015. Tactical network planning for food aid distribution in Kenya. Computers \& Operations Research 56 (2015), 68-83.

[666] Heather Randell and Clark Gray. 2016. Climate variability and educational attainment: Evidence from rural Ethiopia. Global environmental change 41 (2016), 111-123.

[667] Heather Randell and Clark Gray. 2019. Climate change and educational attainment in the global tropics. Proceedings of the National Academy of Sciences 116, 18 (2019), 8840-8845.

[668] Philip J. Rasch, Simone Tilmes, Richard P. Turco, Alan Robock, Luke Oman, Chih-Chieh Chen, Georgiy L. Stenchikov, and Rolando R. Garcia. 2008. An overview of geoengineering of climate using stratospheric sulphate aerosols. Philosophical Transactions of the Royal Society A: Mathematical, Physical and Engineering Sciences 366, 1882 (2008), 4007-4037. 
[669] Parisa Rashidi and Diane J. Cook. 2009. Keeping the resident in the loop: Adapting the smart home to the user. IEEE Transactions on Systems, Man, and Cybernetics, Part A 39, 5 (2009), 949-959.

[670] Stephan Rasp, Michael S. Pritchard, and Pierre Gentine. 2018. Deep learning to represent subgrid processes in climate models. Proceedings of the National Academy of Sciences 115, 39 (2018), 1-6.

[671] Pei-Luen Patrick Rau. 2018. Cross-Cultural Design. Applications in Cultural Heritage, Creativity and Social Development: 10th International Conference, CCD 2018, Held as Part of HCI International 2018, Las Vegas, NV, USA, July 15-20, 2018, Proceedings. Vol. 10912. Springer.

[672] Daniele Ravi, Charence Wong, Benny Lo, and Guang-Zhong Yang. 2017. A deep learning approach to on-node sensor data analytics for mobile or wearable devices. IEEE fournal of Biomedical and Health Informatics 21, 1 (2017), 56-64.

[673] Muhammad Qamar Raza and Abbas Khosravi. 2015. A review on artificial intelligence based load demand forecasting techniques for smart grid and buildings. Renewable and Sustainable Energy Reviews 50 (2015), 1352-1372.

[674] Gerald Rebitzer, Tomas Ekvall, Rolf Frischknecht, Davis Hunkeler, G. Norris, Tomas Rydberg, W.-P. Schmidt, Sangwon Suh, B. Pennington Weidema, and David W. Pennington. 2004. Life cycle assessment: Part 1: Framework, goal and scope definition, inventory analysis, and applications. Environment International 30, 5 (2004), 701-720.

[675] Robert Regue and Will Recker. 2014. Proactive vehicle routing with inferred demand to solve the bikesharing rebalancing problem. Transportation Research Part E: Logistics and Transportation Review 72 (2014), 192-209.

[676] Markus Reichstein, Gustau Camps-Valls, Bjorn Stevens, Martin Jung, Joachim Denzler, Nuno Carvalhais, and Prabhat. 2019. Deep learning and process understanding for data-driven Earth system science. Nature 566, 7743 (2019), 195-204.

[677] Christoph F. Reinhart and Carlos Cerezo Davila. 2016. Urban building energy modeling-A review of a nascent field. Building and Environment 97 (2016), 196-202.

[678] Ali Reza Reisi, Mohammad Hassan Moradi, and Shahriar Jamasb. 2013. Classification and comparison of maximum power point tracking techniques for photovoltaic system: A review. Renewable and Sustainable Energy Reviews 19 (2013), 433-443.

[679] T. Remani, E. A. Jasmin, and T. P. Imthias Ahamed. 2019. Residential load scheduling with renewable generation in the smart grid: A reinforcement learning approach. IEEE Systems fournal 13, 9 (2019), 3283-3294.

[680] Hongyu Ren, Russell Stewart, Jiaming Song, Volodymyr Kuleshov, and Stefano Ermon. 2018. Learning with weak supervision from physics and data-driven constraints. AI Magazine 39, 1 (2018), 27-38.

[681] Restor. 2021. Restor. Retrieved from https://restor.eco/.

[682] J. Rhee, J. Im, and S. Park. 2016. Drought forecasting based on machine learning of remote sensing and long-range forecast data. APEC Climate Center, Republic of Korea.

[683] Ana Carolina Riekstin, Antoine Langevin, Thomas Dandres, Ghyslain Gagnon, and Mohamed Cheriet. 2020. Time series-based GHG emissions prediction for smart homes. IEEE Transactions on Sustainable Computing 5, 1 (2020), 134-146.

[684] E. S. Rigas, S. D. Ramchurn, and N. Bassiliades. 2015. Managing electric vehicles in the smart grid using artificial intelligence: A survey. IEEE Transactions on Intelligent Transportation Systems 16, 4 (2015), 1619-1635.

[685] Christophe Rizet, Eric Cornélis, Michael Browne, and Jacques Léonardi. 2010. GHG emissions of supply chains from different retail systems in Europe. Procedia-Social and Behavioral Sciences 2, 3 (2010), 6154-6164.

[686] Benjamin A. Rizkin, Karina Popovich, and Ryan L. Hartman. 2019. Artificial Neural Network control of thermoelectrically-cooled microfluidics using computer vision based on IR thermography. Computers \& Chemical Engineering 121 (2019), 584-593.

[687] G. Philip Robertson and Peter M. Vitousek. 2009. Nitrogen in agriculture: Balancing the cost of an essential resource. Annual Review of Environment and Resources 34, 1 (2009), 97-125.

[688] Joel Robertson and Del J. DeHart. 2010. An agile and accessible adaptation of Bayesian inference to medical diagnostics for rural health extension workers. In 2010 AAAI Spring Symposium Series.

[689] Caleb Robinson, Bistra Dilkina, Jeffrey Hubbs, Wenwen Zhang, Subhrajit Guhathakurta, Marilyn A. Brown, and Ram M. Pendyala. 2017. Machine learning approaches for estimating commercial building energy consumption. Applied Energy 208 (2017), 889-904.

[690] Carmenza Robledo-Abad, Hans-Jörg Althaus, Göran Berndes, Simon Bolwig, Esteve Corbera, Felix Creutzig, John Garcia-Ulloa, Anna Geddes, Jay S. Gregg, Helmut Haberl, Susanne Hanger, Richard J. Harper, Carol Hunsberger, Rasmus K. Larsen, Christian Lauk, Stefan Leitner, Johan Lilliestam, Hermann Lotze-Campen, Bart Muys, Maria Nordborg, Maria Ölund, Boris Orlowsky, Alexander Popp, Joana Portugal-Pereira, Jürgen Reinhard, Lena Scheiffle, and Pete Smith. 2017. Bioenergy production and sustainable development: Science base for policymaking remains limited. GCB Bioenergy 9, 3 (2017), 541-556.

[691] Alan Robock, Douglas G. MacMartin, Riley Duren, and Matthew W. Christensen. 2013. Studying geoengineering with natural and anthropogenic analogs. Climatic Change 121, 3 (2013), 445-458. 
[692] Daivi Rodima-Taylor. 2012. Social innovation and climate adaptation: Local collective action in diversifying Tanzania. Applied Geography 33 (2012), 128-134.

[693] Pedro Rodríguez-Veiga, James Wheeler, Valentin Louis, Kevin Tansey, and Heiko Balzter. 2017. Quantifying forest biomass carbon stocks from space. Current Forestry Reports 3, 1 (2017), 1-18.

[694] Ido Roll, Daniel M. Russell, and Dragan Gašević. 2018. Learning at Scale. International fournal of Artificial Intelligence in Education 28, 4 (2018), 471-477.

[695] Cristóbal Romero, Sebastián Ventura, Pedro G. Espejo, and César Hervás. 2008. Data mining algorithms to classify students. In 1st International Conference on Educational Data Mining.

[696] Joseph Romm. 2018. Climate Change: What Everyone Needs to Know. Oxford University Press.

[697] Sherri Rose. 2013. Mortality risk score prediction in an elderly population using machine learning. American fournal of Epidemiology 177, 5 (2013), 443-452.

[698] Cynthia Rosenzweig, Joshua Elliott, Delphine Deryng, Alex C. Ruane, Christoph Müller, Almut Arneth, Kenneth J. Boote, Christian Folberth, Michael Glotter, Nikolay Khabarov, Kathleen Neumann, Franziska Piontek, Thomas A. M. Pugh, Erwin Schmid, Elke Stehfest, Hong Yang, and James W. Jones. 2014. Assessing agricultural risks of climate change in the 21st century in a global gridded crop model intercomparison. Proceedings of the National Academy of Sciences 111, 9 (2014), 3268-3273.

[699] Raphael A. Viscarra Rossel and Johan Bouma. 2016. Soil sensing: A new paradigm for agriculture. Agricultural Systems 148 (2016), 71-74.

[700] Michael Rothschild. 1974. A two-armed bandit theory of market pricing. Journal of Economic Theory 9, 2 (1974), 185-202.

[701] Edward S. Rubin, John E. Davison, and Howard J. Herzog. 2015. The cost of CO2 capture and storage. International Journal of Greenhouse Gas Control 40 (2015), 378-400.

[702] Cynthia Rudin, David Waltz, Roger N. Anderson, Albert Boulanger, Ansaf Salleb-Aouissi, Maggie Chow, Haimonti Dutta, Philip N. Gross, Bert Huang, Steve Ierome, Delfina Isaac, Arthur Kressner, Rebecca J. Passonneau, Axinia Radeva, and Leon Wu. 2012. Machine learning for the New York City power grid. IEEE transactions on pattern analysis and machine intelligence 34, 2 (2012), 328-345.

[703] Derek Ruths and Jürgen Pfeffer. 2014. Social media for large studies of behavior. Science 346, 6213 (2014), $1063-1064$.

[704] Daniel F. Salas and Warren B. Powell. 2018. Benchmarking a scalable approximate dynamic programming algorithm for stochastic control of grid-level energy storage. INFORMS fournal on Computing 30, 1 (2018), 106-123.

[705] Marcel Salathe, Linus Bengtsson, Todd J. Bodnar, Devon D. Brewer, John S. Brownstein, Caroline Buckee, Ellsworth M. Campbell, Ciro Cattuto, Shashank Khandelwal, Patricia L. Mabry, and Alessandro Vespignani. 2012. Digital epidemiology. PLoS Computational Biology 8, 7 (2012), e1002616.

[706] Amir Samimi, Kazuya Kawamura, and Abolfazl Mohammadian. 2011. A behavioral analysis of freight mode choice decisions. Transportation Planning and Technology 34, 8 (2011), 857-869.

[707] David Sandalow, Julio Friedmann, and Colin McCormick. 2018. Direct air capture of carbon dioxide: ICEF roadmap 2018. Retrieved from https:/www.icef-forum.org/pdf2018/roadmap/ICEF2018_Roadmap_Draft_for_Comment_ 20181012.pdf. (2018).

[708] Tuomas Sandholm. 1980. Very-Large-Scale Generalized Combinatorial Multi-Attribute Auctions: Lessons from Conducting $\$ 60$ Billion of Sourcing. Carnegie Mellon University.

[709] Lindsay Sanneman, Christopher Fourie, and Julie A. Shah. 2021. The State of Industrial Robotics: Emerging Technologies, Challenges, and Key Research Directions. Now Publishers Foundations and Trends.

[710] M. C. Sarofim, Shubhayu Saha, M. D. Hawkins, D. M. Mills, Jeremy J. Hess, Radley M. Horton, Patrick L. Kinney, Joel D. Schwartz, and Alexis St Juliana. 2016. The Impacts of Climate Change on Human Health in the United States: A Scientific Assessment. U.S. Global Change Research Program, Washington, DC.

[711] Rebecca Scafutto and Carlos de Souza Filho. 2018. Detection of methane plumes using airborne midwave infrared (3-5 $\mu \mathrm{m}$ ) hyperspectral data. Remote Sensing 10, 8 (2018), 1237.

[712] R. Schaeffer, R. Sims, J. Corfee-Morlot, F. Creutzig, X. Cruz-Nunez, D. Dimitriu, and M. D’Agosto. 2014. Transport, in IPCC, Working Group III Contribution to the Fifth Assessment Report of the Intergovernmental Panel on Climate Change, Climate Change 2014: Mitigation of Climate Change, Chapter 8. Geneva. O. Edenhofer, R. Pichs-Madruga, Y. Sokona, E. Farahani, S. Kadner, K. Seyboth, A. Adler, I. Baum, S. Brunner, P. Eickemeier, B. Kriemann, J. Savolainen, S. Schlömer, C. von Stechow, T. Zwickel, and J. C. Minx (Eds.). Cambridge University Press, Cambridge.

[713] Andreas W. Schäfer, Antony D. Evans, Tom G. Reynolds, and Lynnette Dray. 2015. Costs of mitigating CO2 emissions from passenger aircraft. Nature Climate Change 6 (2015), 412-417.

[714] Simon Scheidegger and Ilias Bilionis. 2019. Machine learning for high-dimensional dynamic stochastic economies. Journal of Computational Science 33 (2019), 68-82.

[715] Maja Schlüter, Alessandro Tavoni, and Simon Levin. 2016. Robustness of norm-driven cooperation in the commons. Proceedings of the Royal Society B: Biological Sciences 283, 1822 (2016), 20152431. 
[716] Victor Schmidt, Alexandra Luccioni, S. Karthik Mukkavilli, Narmada Balasooriya, Kris Sankaran, Jennifer Chayes, and Yoshua Bengio. 2019. Visualizing the Consequences of Climate Change Using Cycle-Consistent Adversarial Networks. In ICLR AI for Social Good Workshop.

[717] Tapio Schneider, Shiwei Lan, Andrew Stuart, and João Teixeira. 2017. Earth system modeling 2.0: A blueprint for models that learn from observations and targeted high-resolution simulations. Geophysical Research Letters 44, 24 (2017), 12396-12417.

[718] R. D. Schuiling and P. Krijgsman. 2006. Enhanced weathering: An effective and cheap tool to sequester CO2. Climatic Change 74, 1-3 (2006), 349-354.

[719] Joel Schwartz, Jonathan M. Samet, and Jonathan A. Patz. 2004. Hospital admissions for heart disease: The effects of temperature and humidity. Epidemiology 15, 6 (2004), 755-761.

[720] Roy Schwartz, Jesse Dodge, Noah A. Smith, and Oren Etzioni. 2019. Green AI. Commun. ACM 63, 12 (December 2020), 54-63.

[721] F. C. Schweppe, B. Daryanian, and R. D. Tabors. 1989. Algorithms for a spot price responding residential load controller. IEEE Transactions on Power Systems 4, 2 (1989), 507-516.

[722] L. Scime and J. Beuth. 2018. Anomaly detection and classification in a laser powder bed additive manufacturing process using a trained computer vision algorithm. Additive Manufacturing 19 (2018), 114-126.

[723] Sense. 2021. Sense. Retrieved from https://sense.com.

[724] Toru Seo, Takahiko Kusakabe, Hiroto Gotoh, and Yasuo Asakura. 2017. Interactive online machine learning approach for activity-travel survey. Transportation Research Part B: Methodological 123, (2017), 362-373.

[725] Kristen A. Severson, Peter M. Attia, Norman Jin, Nicholas Perkins, Benben Jiang, Zi Yang, Michael H. Chen, Muratahan Aykol, Patrick K. Herring, Dimitrios Fraggedakis, Martin Z. Bazant, Stephen J. Harris, William C. Chueh, and Richard D. Braatz. 2019. Data-driven prediction of battery cycle life before capacity degradation. Nature Energy 4, (2019), 383-391.

[726] Bobak Shahriari, Kevin Swersky, Ziyu Wang, Ryan P. Adams, and Nando De Freitas. 2015. Taking the human out of the loop: A review of Bayesian optimization. Proceedings of the IEEE 104, 1 (2015), 148-175.

[727] Chaopeng Shen. 2018. A trans-disciplinary review of deep learning research for water resources scientists. Water Resources Research 54, 11 (2018), 8558-8593.

[728] John G. Shepherd. 2009. Geoengineering the Climate: Science, Governance and Uncertainty. Royal Society.

[729] Steven C. Sherwood, Sandrine Bony, and Jean-Louis Dufresne. 2014. Spread in model climate sensitivity traced to atmospheric convective mixing. Nature 505 (2014), 37-42.

[730] S. A. Shevchik, C. Kenel, C. Leinenbach, and K. Wasmer. 2018. Acoustic emission for in situ quality monitoring in additive manufacturing using spectral convolutional neural networks. Additive Manufacturing 4 (2018), 383-391.

[731] Linda Shi, Eric Chu, Isabelle Anguelovski, Alexander Aylett, Jessica Debats, Kian Goh, Todd Schenk, Karen C. Seto, David Dodman, Debra Roberts, J. Timmons Roberts, and Stacy D. VanDeveer. 2016. Roadmap towards justice in urban climate adaptation research. Nature Climate Change 6, 2 (2016), 131.

[732] Qinru Shi, Jonathan M. Gomes-Selman, Roosevelt García-Villacorta, Suresh Sethi, Alexander S. Flecker, and Carla P. Gomes. 2018. Efficiently optimizing for dendritic connectivity on tree-structured networks in a multi-objective framework. In 1st ACM SIGCAS Conference on Computing and Sustainable Societies (COMPASS'18). ACM, New York, NY.

[733] Kyriacos Shiarlis, Joao Messias, Maarten van Someren, Shimon Whiteson, Jaebok Kim, Jered Hendrik Vroon, Gwenn Englebienne, Khiet Phuong Truong, Noé Pérez-Higueras, Ignacio Pérez-Hurtado, Rafael Ramon-Vigo, Fernando Caballero, Luis Merino, Jie Shen, Stavros Petridis, Maja Pantic, Lasse Hedman, Marten Scherlund, Raphaël Koster, and Hervé Michel. 2015. TERESA: A socially intelligent semi-autonomous telepresence system. In Workshop on Machine Learning for Social Robotics.

[734] J. Shukla. 1998. Predictability in the midst of chaos: A scientific basis for climate forecasting. Science 282, 5389 (1998), 728-731.

[735] Xiao-Sheng Si, Wenbin Wang, Chang-Hua Hu, and Dong-Hua Zhou. 2011. Remaining useful life estimation-a review on the statistical data driven approaches. European fournal of Operational Research 213, 1 (2011), 1-14.

[736] Glenn Gregory Sias. 2017. Characterization of the Life Cycle Environmental Impacts and Benefits of Smart Electric Meters and Consequences of their Deployment in California. Ph.D. Dissertation. UCLA.

[737] Mafalda Silva, Vítor Leal, Vítor Oliveira, and Isabel M. Horta. 2018. A scenario-based approach for assessing the energy performance of urban development pathways. Sustainable Cities and Society 40 (2018), 372-382.

[738] David Silver, Aja Huang, Christopher J. Maddison, Arthur Guez, Laurent Sifre, George van den Driessche, Julian Schrittwieser, Ioannis Antonoglou, Veda Panneershelvam, Marc Lanctot, Sander Dieleman, Dominik Grewe, John Nham, Nal Kalchbrenner, Ilya Sutskever, Timothy Lillicrap, Madeleine Leach, Koray Kavukcuoglu, Thore Graepel, and Demis Hassabis. 2016. Mastering the game of Go with deep neural networks and tree search. Nature 529 (2016), 484-503. 
[739] Adish Singla, Marco Santoni, Gÿbor Bartók, Pratik Mukerji, Moritz Meenen, and Andreas Krause. 2015. Incentivizing users for balancing bike sharing systems. In 29th AAAI Conference on Artificial Intelligence (AAAI'15). AAAI Press, 723-729.

[740] Muhammed Sit and Ibrahim Demir. 2019. Decentralized flood forecasting using deep neural networks. Preprint arXiv:1902.02308 (2019).

[741] Andrew Small and Laura Bliss. 2019. The race to code the curb. Citylab. Retrieved from https://www.citylab.com/ transportation/2019/04/smart-cities-maps-curb-data-coord-sidewalk-tech-street-design/586177/.

[742] Small Robot Company. 2021. Small Robot Company. Retrieved from https://www.smallrobotcompany.com/.

[743] Jordan P. Smith, John A. Dykema, and David W. Keith. 2018. Production of sulfates onboard an aircraft: Implications for the cost and feasibility of stratospheric solar geoengineering. Earth and Space Science 5, 4 (2018), 150-162.

[744] Sandra Ó. Snæbjörnsdóttir and Sigurdur R. Gislason. 2016. CO2 storage potential of basaltic rocks offshore Iceland. Energy Procedia 86 (2016), 371-380.

[745] Jasper Snoek, Hugo Larochelle, and Ryan P. Adams. 2012. Practical Bayesian optimization of machine learning algorithms. In 25th International Conference on Neural Information Processing Systems - Volume 2 (NIPS'12). Curran Associates Inc., 2951-2959.

[746] Iman Soleimanmeigouni, Alireza Ahmadi, and Uday Kumar. 2018. Track geometry degradation and maintenance modelling: A review. Proceedings of the Institution of Mechanical Engineers, Part F: Fournal of Rail and Rapid Transit 232, 1 (2018), 73-102.

[747] Lars Wilko Sommer, Tobias Schuchert, and Jürgen Beyerer. 2017. Fast deep vehicle detection in aerial images. In 2017 IEEE Winter Conference on Applications of Computer Vision (WACV'17). IEEE, 311-319.

[748] Steve Sorrell. 2009. Jevons' Paradox revisited: The evidence for backfire from improved energy efficiency. Energy Policy 37, 4 (2009), 1456-1469.

[749] Southwest Research Institute. 2016. SwRI Developing Methane Leak Detection System for DOE. Retrieved from https://www.swri.org/press-release/swri-developing-methane-leak-detection-system-doe.

[750] D. Srinivasan, W. S. Ng, and A. C. Liew. 2006. Neural-network-based signature recognition for harmonic source identification. IEEE Transactions on Power Delivery 21, 1 (2006), 398-405.

[751] Satyam Srivastava, Saikrishna Vaddadi, Pankaj Kumar, and Shashikant Sadistap. 2018. Design and development of reverse osmosis (RO) plant status monitoring system for early fault prediction and predictive maintenance. Applied Water Science 8, 6 (2018), 159.

[752] Elizabeth Stanny and Kirsten Ely. 2008. Corporate environmental disclosures about the effects of climate change. Corporate Social Responsibility and Environmental Management 15, 6 (2008), 338-348.

[753] William Steinhurst, Patrick Knight, and Melissa Schultz. 2012. Hydropower greenhouse gas emissions. Conservation Law Foundation 24 (2012), 6

[754] William Steinhurst, Patrick Knight, and Melissa Schultz. 2012. Hydropower Greenhouse Gas Emissions: State of the Research. Synapse Energy Economics, Inc. https:/www.nrc.gov/docs/ML1209/ML12090A850.pdf.

[755] Nicholas Stern. 2008. The economics of climate change. American Economic Review 98, 2 (2008), 1-37.

[756] Thomas Sterner, Edward B. Barbier, Ian Bateman, Inge van den Bijgaart, Anne-Sophie Crépin, Ottmar Edenhofer, Carolyn Fischer, Wolfgang Habla, John Hassler, Olof Johansson-Stenman, Andreas Lange, Stephen Polasky, Johan Rockström, Henrik G. Smith, Will Steffen, Gernot Wagner, James E. Wilen, Francisco Alpízar, Christian Azar, Donna Carless, Carlos Chávez, Jessica Coria, Gustav Engström, Sverker C. Jagers, Gunnar Köhlin, Åsa Löfgren, Håkan Pleijel, and Amanda Robinson. 2019. Policy design for the Anthropocene. Nature Sustainability 2, 1 (2019), 14-21.

[757] High-Level Commission on Carbon Prices. 2017. Report of the high-level commission on carbon prices. World Bank Publications.

[758] Joshuah K. Stolaroff, Constantine Samaras, Emma R. O’Neill, Alia Lubers, Alexandra S. Mitchell, and Daniel Ceperley. 2018. Energy use and life cycle greenhouse gas emissions of drones for commercial package delivery. Nature Communications 9, 1 (2018), 409.

[759] Trude Storelvmo, W. R. Boos, and N. Herger. 2014. Cirrus cloud seeding: A climate engineering mechanism with reduced side effects? Philosophical Transactions of the Royal Society A: Mathematical, Physical and Engineering Sciences 372, 2031 (2014), 20140116.

[760] Goran Strbac. 2008. Demand side management: Benefits and challenges. Energy Policy 36, 12 (2008), $4419-4426$.

[761] Dalia Streimikiene and Tomas Balezentis. 2013. Multi-objective ranking of climate change mitigation policies and measures in Lithuania. Renewable and Sustainable Energy Reviews 18 (2013), 144-153.

[762] Yolande Strengers. 2015. Meeting in the global workplace: Air travel, telepresence and the body. Mobilities 10, 4 (2015), 592-608.

[763] E. Strobach and G. Bel. 2015. Improvement of climate predictions and reduction of their uncertainties using learning algorithms. Atmospheric Chemistry and Physics 15 (2015), 8631-8641. 
[764] Emma Strubell, Ananya Ganesh, and Andrew McCallum. 2019. Energy and policy considerations for deep learning in NLP. In Proceedings of the Association for Computational Linguistics.

[765] Anissa Yuniashaesa Suatmadi, Felix Creutzig, and Ilona Otto. 2019. On-demand motorcycle taxis improve mobility, not sustainability. Case Studies on Transport Policy 7, 2 (2019).

[766] Salah Sukkarieh. 2017. Mobile on-farm digital technology for smallholder farmers. 218-229. Technical Report.

[767] Brian L. Sullivan, Christopher L. Wood, Marshall J. Iliff, Rick E. Bonney, Daniel Fink, and Steve Kelling. 2009. eBird: A citizen-based bird observation network in the biological sciences. Biological Conservation 142, 10 (2009), 2282-2292.

[768] Chong Sun, Nader Azari, and Chintan Turakhia. 2020. Gallery: A Machine Learning Model Management System at Uber. In 22nd International Conference on Extending Database Technology (EDBT'20). 474-485.

[769] Wei Sun and Chongchong Zhang. 2018. Analysis and forecasting of the carbon price using multi-resolution singular value decomposition and extreme learning machine optimized by adaptive whale optimization algorithm. Applied Energy 231 (2018), 1354-1371.

[770] Yanshuo Sun, Zhibin Jiang, Jinjing Gu, Min Zhou, Yeming Li, and Lei Zhang. 2018. Analyzing high speed rail passengers' train choices based on new online booking data in China. Transportation Research Part C: Emerging Technologies 97 (2018), 96-113.

[771] Yuchi Sun, Gergely Szűcs, and Adam R. Brandt. 2018. Solar PV output prediction from video streams using convolutional neural networks. Energy \& Environmental Science 11, 7 (2018), 1811-1818.

[772] Vasughi Sundramoorthy, Grahame Cooper, Nigel Linge, and Qi Liu. 2011. Domesticating energy-monitoring systems: Challenges and design concerns. IEEE Pervasive Computing 10, 1 (2011), 20-27.

[773] Santosh K. Suram, Yexiang Xue, Junwen Bai, Ronan Le Bras, Brendan Rappazzo, Richard Bernstein, Johan Bjorck, Lan Zhou, R. Bruce van Dover, Carla P. Gomes, and John M. Gregoire. 2016. Automated phase mapping with AgileFD and its application to light absorber discovery in the $\mathrm{V}-\mathrm{Mn}-\mathrm{Nb}$ oxide system. ACS Combinatorial Science 19,1 (2016), 37-46.

[774] Vanessa Svihla and Marcia C. Linn. 2012. A design-based approach to fostering understanding of global climate change. International fournal of Science Education 34, 5 (2012), 651-676.

[775] Ali Rahim Taleqani, Jill Hough, and Kendall E. Nygard. 2019. Public opinion on dockless bike sharing: A machine learning approach. Transportation Research Record 2673, 4 (2019), 195-204.

[776] Liang Tang, Chenfeng Xiong, and Lei Zhang. 2018. Spatial transferability of neural network models in travel demand modeling. Journal of Computing in Civil Engineering 32, 3 (2018), 04018010.

[777] Fei Tao, Jiangfeng Cheng, Qinglin Qi, Meng Zhang, He Zhang, and Fangyuan Sui. 2018. Digital twin-driven product design, manufacturing and service with big data. The International fournal of Advanced Manufacturing Technology 94, 9-12 (2018), 3563-3576.

[778] Lan Tao, Elizabeth Garnsey, David Probert, and Tom Ridgman. 2010. Innovation as response to emissions legislation: Revisiting the automotive catalytic converter at Johnson Matthey. R\&d Management 40, 2 (2010), 154-168.

[779] Ye Tao, Miaohua Huang, and Lan Yang. 2018. Data-driven optimized layout of battery electric vehicle charging infrastructure. Energy 150 (2018), 735-744.

[780] R. Tavakoli and Z. Pantic. 2017. ANN-based algorithm for estimation and compensation of lateral misalignment in dynamic wireless power transfer systems for EV charging. In 2017 IEEE Energy Conversion Congress and Exposition (ECCE'17). 2602-2609.

[781] K. E. Taylor, R. J. Stouffer, and G. A. Meehl. 2012. An overview of CMIP5 and the experiment design. Bulletin of the American Meteorological Society 93, 4 (2012), 485-498.

[782] Claudia Tebaldi and Reto Knutti. 2007. The use of the multi-model ensemble in probabilistic climate projections. Philosophical Transactions of the Royal Society A 365 (2007), 2053-2075.

[783] C. Tebaldi and D. B. Lobell. 2008. Towards probabilistic projections of climate change impacts on global crop yields. Geophysical Research Letters 35, 8 (2008).

[784] Jacob Teter, Pierpaolo Cazzola, and Timur Gül. 2017. The Future of Trucks. International Energy Agency.

[785] The World Bank. 2017. Agriculture, forestry, and fishing, value added. Retrieved from https://data.worldbank.org/ indicator/NV.AGR.TOTL.CD.

[786] Jayaraman Thiagarajan, Nikhil Jain, Rushil Anirudh, Alfredo Giminez, Rahul Sridhar, Marathe Aniruddha, Tao Wang, Mural Emani, Abhinav Bhatele, and Todd Gamblin. 2018. Bootstrapping parameter space exploration for fast tuning. In 2018 International Conference on Supercomputing. 385-395.

[787] Jule Thober, Nina Schwarz, and Kathleen Hermans. 2018. Agent-based modeling of environment-migration linkages: A review. Ecology and Society 23, 2 (2018).

[788] Thorvald. 2021. Thorvald. Retrieved from https://sagarobotics.com/.

[789] Tomorrow. 2019. electricityMap. Retrived from https://www.electricitymap.org.

[790] Tomorrow. 2019. Tomorrow. Retrieved from https://www.tmrow.com/. 
[791] Benjamin A. Toms, Elizabeth A. Barnes, , and Imme Ebert-Uphoff. 2020. Physically interpretable neural networks for the geosciences: Applications to earth system variability. fournal of Advances in Modeling Earth Systems 12, 9 (2020), e2019MS002002.

[792] Fan Tong, Paulina Jaramillo, and Inês M. L. Azevedo. 2015. Comparison of life cycle greenhouse gases from natural gas pathways for medium and heavy-duty vehicles. Environmental Science \& Technology 49, 12 (2015), 7123-7133.

[793] N. Topping. 2019. Is your company ready for a zero-carbon future? Retrived from https://hbr.org/2019/06/is-your company-ready-for-a-zero-carbon-future.

[794] Nicholas W. Touran, John Gilleland, Graham T. Malmgren, Charles Whitmer, and William H. Gates III. 2017. Computational tools for the integrated design of advanced nuclear reactors. Engineering 3, 4 (2017), 518-526.

[795] Calvin P. Tribby, Harvey J. Miller, Barbara B. Brown, Carol M. Werner, and Ken R. Smith. 2017. Analyzing walking route choice through built environments using random forests and discrete choice techniques. Environment and Planning B: Urban Analytics and City Science 44, 6 (2017), 1145-1167.

[796] Ioannis Tsapakis and William H. Schneider. 2015. Use of support vector machines to assign short-term counts to seasonal adjustment factor groups. Transportation Research Record: Journal of the Transportation Research Board 2527 (2015), 8-17.

[797] Grigorios Tsoumakas. 2019. A survey of machine learning techniques for food sales prediction. Artificial Intelligence Review 52, 1 (2019), 441-447.

[798] Wei Tu, Jinzhou Cao, Yang Yue, Shih-Lung Shaw, Meng Zhou, Zhensheng Wang, Xiaomeng Chang, Yang Xu, and Qingquan Li. 2017. Coupling mobile phone and social media data: A new approach to understanding urban functions and diurnal patterns. International fournal of Geographical Information Science 31, 12 (2017), 2331-2358.

[799] Gustavo M. Ugarte, Jay S. Golden, and Kevin J. Dooley. 2016. Lean versus green: The impact of lean logistics on greenhouse gas emissions in consumer goods supply chains. Journal of Purchasing and Supply Management 22, 2 (2016), 98-109.

[800] Mitsutaro Umehara, Helge S. Stein, Dan Guevarra, Paul F. Newhouse, David A. Boyd, and John M. Gregoire. 2019. Analyzing machine learning models to accelerate generation of fundamental materials insights. npj Computational Materials 5, 1 (2019), 34.

[801] UNESCO. 2015. Not fust Hot Air: Putting Climate Change Education into Practice. United Nations Educational, Scientific and Cultural Organization.

[802] Diana Urge-Vorsatz, Ksenia Petrichenko, Maja Staniec, and Jiyong Eom. 2013. Energy use in buildings in a long-term perspective. Current Opinion in Environmental Sustainability 5, 2 (2013), 141-151.

[803] U.S. Department of Energy. 2012. Fuel Cell Technologies Office Multi-Year Research, Development, and Demonstration Plan. Retrieved from https:/www.energy.gov/eere/fuelcells/downloads/fuel-cell-technologies-office-multiyear-research-development-and- 22 .

[804] Lorenzo Valerio, Andrea Passarella, and Marco Conti. 2016. Hypothesis transfer learning for efficient data computing in smart cities environments. In 2016 IEEE International Conference on Smart Computing (SMARTCOMP'16). IEEE, 1-8.

[805] Jan C. van Gemert, Camiel R. Verschoor, Pascal Mettes, Kitso Epema, Lian Pin Koh, and Serge Wich. 2014. Nature conservation drones for automatic localization and counting of animals. In European Conference on Computer Vision. Springer, 255-270.

[806] Grant Van Horn, Steve Branson, Ryan Farrell, Scott Haber, Jessie Barry, Panos Ipeirotis, Pietro Perona, and Serge Belongie. 2015. Building a bird recognition app and large scale dataset with citizen scientists: The fine print in fine-grained dataset collection. In IEEE Conference on Computer Vision and Pattern Recognition. 595-604.

[807] Grant Van Horn and Pietro Perona. 2017. The devil is in the tails: Fine-grained classification in the wild. Preprint arXiv:1709.01450 (2017).

[808] Annelies Vandermeulen, Bram van der Heijde, and Lieve Helsen. 2018. Controlling district heating and cooling networks to unlock flexibility: A review. Energy 151 (2018), 103-115.

[809] Vivek K. Varma, Ian Ferguson, and Ian Wild. 2000. Decision support system for the sustainable forest management. Forest Ecology and Management 128, 1 (2000), 49-55.

[810] José Vázquez-Canteli and Zoltán Nagy. 2019. Reinforcement learning for demand response: A review of algorithms and modeling techniques. Applied Energy 235 (2019), 1072-1089.

[811] Jesús Vega, Sebastián Dormido-Canto, Juan M. López, Andrea Murari, Jesús M. Ramírez, Raúl Moreno, Mariano Ruiz, Diogo Alves, Robert Felton, and JET-EFDA Contributors. 2013. Results of the JET real-time disruption predictor in the ITER-like wall campaigns. Fusion Engineering and Design 88, 6-8 (2013), 1228-1231.

[812] Giuseppe A. Veltri and Dimitrinka Atanasova. 2017. Climate change on Twitter: Content, media ecology and information sharing behaviour. Public Understanding of Science 26, 6 (2017), 721-737.

[813] Subhashini Venugopalan and Varun Rai. 2015. Topic based classification and pattern identification in patents. Technological Forecasting and Social Change 94 (2015), 236-250.

[814] David G. Victor. 2019. How artificial intelligence will affect the future of energy and climate. Retrieved from https://www.brookings.edu/research/how-artificial-intelligence-will-affect-the-future-of-energy-and-climate/. 
[815] Jackson Voelkel, Vivek Shandas, and Brendon Haggerty. 2016. Developing high-resolution descriptions of urban heat islands: A public health imperative. Preventing Chronic Disease 13, 9 (2016).

[816] Stefan Voigt, Thomas Kemper, Torsten Riedlinger, Ralph Kiefl, Klaas Scholte, and Harald Mehl. 2007. Satellite image analysis for disaster and crisis-management support. IEEE Transactions on Geoscience and Remote Sensing 45, 6 (2007), 1520-1528.

[817] Alexandra Von Meier. 2006. Electric Power Systems: A Conceptual Introduction. Wiley Online Library.

[818] Cyril Voyant, Gilles Notton, Soteris Kalogirou, Marie-Laure Nivet, Christophe Paoli, Fabrice Motte, and Alexis Fouilloy. 2017. Machine learning methods for solar radiation forecasting: A review. Renewable Energy 105 (2017), 569-582.

[819] Wladislaw Waag, Christian Fleischer, and Dirk Uwe Sauer. 2014. Critical review of the methods for monitoring of lithium-ion batteries in electric and hybrid vehicles. Journal of Power Sources 258 (2014), 321-339.

[820] Zia Wadud, Don MacKenzie, and Paul Leiby. 2016. Help or hindrance? The travel, energy and carbon impacts of highly automated vehicles. Transportation Research Part A: Policy and Practice 86 (2016), 1-18.

[821] Mirwaes Wahabzada, Anne-Katrin Mahlein, Christian Bauckhage, Ulrike Steiner, Erich-Christian Oerke, and Kristian Kersting. 2016. Plant phenotyping using probabilistic topic models: Uncovering the hyperspectral language of plants. Scientific Reports 6 (2016), 22482.

[822] Can Wan, Jian Zhao, Yonghua Song, Zhao Xu, Jin Lin, and Zechun Hu. 2015. Photovoltaic and solar power forecasting for smart grid energy management. CSEE fournal of Power and Energy Systems 1, 4 (2015), 38-46.

[823] Jiangwen Wan, Yang Yu, Yinfeng Wu, Renjian Feng, and Ning Yu. 2012. Hierarchical leak detection and localization method in natural gas pipeline monitoring sensor networks. Sensors 12, 1 (2012), 189-214.

[824] Anna X. Wang, Caelin Tran, Nikhil Desai, David Lobell, and Stefano Ermon. 2018. Deep transfer learning for crop yield prediction with remote sensing data. In 1st ACM SIGCAS Conference on Computing and Sustainable Societies. ACM, 50.

[825] Hao Wang and Baosen Zhang. 2018. Energy storage arbitrage in real-time markets via reinforcement learning. In 2018 IEEE Power \& Energy Society General Meeting (PESGM'18). IEEE, 1-5.

[826] Jingfan Wang, Lyne P. Tchapmi, Arvind P. Ravikumar, Mike McGuire, Clay S. Bell, Daniel Zimmerle, Silvio Savarese, and Adam R. Brandt. 2020. Machine vision for natural gas methane emissions detection using an infrared camera. Applied Energy 257 (2020), 113998.

[827] Shuangyuan Wang, Ran Li, Adrian Evans, and Furong Li. 2019. Electric vehicle load disaggregation based on limited activation matching pursuits. Energy Procedia 158 (2019), 2611-2616.

[828] Xi Wang, Hua Cai, and H. Keith Florig. 2016. Energy-saving implications from supply chain improvement: An exploratory study on China's consumer goods retail system. Energy Policy 95 (2016), 411-420.

[829] Zhanwei Wang, Zhiwei Wang, Suowei He, Xiaowei Gu, and Zeng Feng Yan. 2017. Fault detection and diagnosis of chillers using Bayesian network merged distance rejection and multi-source non-sensor information. Applied Energy 188 (2017), 200-214.

[830] Logan Ward, Ankit Agrawal, Alok Choudhary, and Christopher Wolverton. 2016. A general-purpose machine learning framework for predicting properties of inorganic materials. npj Computational Materials 2 (2016), 16028.

[831] Nick Watts, W. Neil Adger, Sonja Ayeb-Karlsson, Yuqi Bai, Peter Byass, Diarmid Campbell-Lendrum, Tim Colbourn, Peter Cox, Michael Davies, Michael Depledge, et al. 2017. The Lancet Countdown: Tracking progress on health and climate change. The Lancet 389, 10074 (2017), 1151-1164.

[832] WattTime. 2021. WattTime. Retrieved from https://www.watttime.org/.

[833] Chun Wei, Zhe Zhang, Wei Qiao, and Liyan Qu. 2015. Reinforcement-learning-based intelligent maximum power point tracking control for wind energy conversion systems. IEEE Transactions on Industrial Electronics 62, 10 (2015), 6360-6370.

[834] Sun Wei, Zhang Chongchong, and Sun Cuiping. 2018. Carbon pricing prediction based on wavelet transform and K-ELM optimized by bat optimization algorithm in China ETS: The case of Shanghai and Hubei carbon markets. Carbon Management 9, 6 (2018), 605-617.

[835] Max Welling. 2015. Are ML and statistics complementary? In IMS-ISBA Meeting on Data Science in the Next 50 Years.

[836] Gege Wen, Meng Tang, and Sally M. Benson. 2021. Towards a predictor for CO2 plume migration using deep neural networks. International Journal of Greenhouse Gas Control 105 (2021), 103223.

[837] J. Wen, J. Zhao, and P. Jaillet. 2017. Rebalancing shared mobility-on-demand systems: A reinforcement learning approach. In 2017 IEEE 20th International Conference on Intelligent Transportation Systems (ITSC'17). 220-225.

[838] Rafał Weron. 2014. Electricity price forecasting: A review of the state-of-the-art with a look into the future. International fournal of Forecasting 30, 4 (2014), 1030-1081.

[839] Anthony LeRoy Westerling. 2016. Increasing western US forest wildfire activity: Sensitivity to changes in the timing of spring. Philosophical Transactions of the Royal Society B: Biological Sciences 371, 1696 (2016), 20150178.

[840] John Weyant. 2017. Some contributions of integrated assessment models of global climate change. Review of Environmental Economics and Policy 11, 1 (2017), 115-137. 
[841] Ami Wiesel, Avinatan Hassidim, Gal Elidan, Guy Shalev, Mor Schlesinger, Oleg Zlydenko, Ran El-Yaniv, Sella Nevo, Yossi Matias, Yotam Gigi, et al. 2018. Ml for flood forecasting at scale. (2018).

[842] Bryan Wilder, Bistra Dilkina, and Milind Tambe. 2019. Melding the data-decisions pipeline: Decision-focused learning for combinatorial optimization. AAAI Conference on Artificial Intelligence 33, 01 (2019), 1658-1665.

[843] Jared Willard, Xiaowei Jia, Shaoming Xu, Michael Steinbach, and Vipin Kumar. 2020. Integrating physics-based modeling with machine learning: A survey. arXiv preprint arXiv:2003.04919 (2020).

[844] Sella Nevo, Vova Anisimov, Gal Elidan, Ran El-Yaniv, Pete Giencke, Yotam Gigi, Avinatan Hassidim, Zach Moshe, Mor Schlesinger, Guy Shalev, Ajai Tirumali, Ami Wiesel, Oleg Zlydenko, and Yossi Matias. 2019. ML for flood forecasting at scale. Preprint arXiv:1901.09583.

[845] K. Williamson, A. Satre-Meloy, K. Velasco, and K. Green. 2018. Climate Change Needs Behavior Change: Making the Case for Behavioral Solutions to Reduce Global Warming. Technical Report. Center for Behavior and the Environment. Retrived from https://rare.org/wp-content/uploads/2019/02/2018-CCNBC-Report.pdf.

[846] C. G. Windsor, G. Pautasso, C. Tichmann, R. J. Buttery, T. C. Hender, and JET EFDA Contributors. 2005. A cross-tokamak neural network disruption predictor for the JET and ASDEX Upgrade tokamaks. Nuclear Fusion 45, 5 (2005), 337.

[847] Andrew Winston. 2011. Excess inventory wastes carbon and energy, not just money. Harvard Business Review.

[848] Allen J. Wood, Bruce F. Wollenberg, and Gerald B. Sheblé. 2013. Power Generation, Operation, and Control. John Wiley \& Sons.

[849] S. W. Wood and Annette Cowie. 2004. A review of greenhouse gas emission factors for fertiliser production. Climate Technology Centre and Network.

[850] D. Wroblewski, G. L. Jahns, and J. A. Leuer. 1997. Tokamak disruption alarm based on a neural network model of the high-beta limit. Nuclear Fusion 37, 6 (1997), 725.

[851] Cathy Wu, Aboudy Kreidieh, Kanaad Parvate, Eugene Vinitsky, and Alexandre M. Bayen. 2017. Flow: Architecture and benchmarking for reinforcement learning in traffic control. Preprint arXiv:1710.05465 (2017).

[852] Cathy Wu, Aboudy Kreidieh, Eugene Vinitsky, and Alexandre M. Bayen. 2017. Emergent behaviors in mixedautonomy traffic. In 1st Annual Conference on Robot Learning.

[853] Jinsong Wu, Song Guo, Jie Li, and Deze Zeng. 2016. Big data meet green challenges: Big data toward green applications. IEEE Systems fournal 10, 3 (2016), 888-900.

[854] Lifeng Wu, Xiaohui Fu, and Yong Guan. 2016. Review of the remaining useful life prognostics of vehicle lithium-ion batteries using data-driven methodologies. Applied Sciences 6, 6 (2016), 166.

[855] Qunli Wu and Hongjie Zhang. 2019. Research on optimization allocation scheme of initial carbon emission quota from the perspective of welfare effect. Energies 12, 11 (2019), 2118.

[856] Xiaojian Wu, Jonathan Gomes-Selman, Qinru Shi, Yexiang Xue, Roosevelt Garcia-Villacorta, Elizabeth Anderson, Suresh Sethi, Scott Steinschneider, Alexander Flecker, and Carla Gomes. 2018. Efficiently approximating the Pareto Frontier: Hydropower dam placement in the Amazon basin. In 32nd AAAI Conference on Artificial Intelligence.

[857] Matt Wytock and Zico Kolter. 2013. Sparse Gaussian conditional random fields: Algorithms, theory, and application to energy forecasting. In International Conference on Machine Learning. 1265-1273.

[858] Álinson S. Xavier, Feng Qiu, and Shabbir Ahmed. 2020. Learning to solve large-scale security-constrained unit commitment problems. INFORMS fournal on Computing 33, 2 (2020), 419-835.

[859] Tian Xie and Jeffrey C. Grossman. 2018. Crystal graph convolutional neural networks for an accurate and interpretable prediction of material properties. Physical Review Letters 120, 14 (2018), 145301.

[860] Yexiang Xue, Ian Davies, Daniel Fink, Christopher Wood, and Carla P. Gomes. 2016. Avicaching: A two stage game for bias reduction in citizen science. In 2016 International Conference on Autonomous Agents \& Multiagent Systems. International Foundation for Autonomous Agents and Multiagent Systems, 776-785.

[861] Kai-le Zhou, Shan-lin Yang, and Chao Shen. 2013. A review of electric load classification in smart grid environment. Renewable and Sustainable Energy Reviews 24 (2013), 103-110.

[862] William A. Yasnoff, Patrick W. O. Carroll, Denise Koo, Robert W. Linkins, and Edwin M. Kilbourne. 2000. Public health informatics: Improving and transforming public health in the information age. fournal of Public Health Management and Practice 6, 6 (2000), 67-75.

[863] Fredrik Ygge, J. M. Akkermans, Arne Andersson, Marko Krejic, and Erik Boertjes. 1999. The HOMEBOTS system and field test: A multi-commodity market for predictive power load management. In 4th International Conference on the Practical Application of Intelligent Agents and Multi-Agent Technology, Vol. 1. 363-382.

[864] M. Yin, M. Sheehan, S. Feygin, J. Paiement, and A. Pozdnoukhov. 2018. A generative model of urban activities from cellular data. IEEE Transactions on Intelligent Transportation Systems 19, 6 (2018), 1682-1696.

[865] Raul Yondo, Esther Andrés, and Eusebio Valero. 2018. A review on design of experiments and surrogate models in aircraft real-time and many-query aerodynamic analyses. Progress in Aerospace Sciences 96 (2018), 23-61. 
[866] Jiaxuan You, Xiaocheng Li, Melvin Low, David Lobell, and Stefano Ermon. 2017. Deep Gaussian process for crop yield prediction based on remote sensing data. In 31st AAAI Conference on Artificial Intelligence.

[867] Grace Young, Vassileios Balntas, and Victor Prisacariu. 2018. Convolutional neural networks predict fish abundance from underlying coral reef texture. MarXiv. August 31 (2018).

[868] Jiafan Yu, Zhecheng Wang, Arun Majumdar, and Ram Rajagopal. 2018. DeepSolar: A machine learning framework to efficiently construct a solar deployment database in the United States. Foule 2, 12 (2018), 2605-2617.

[869] Emilio Zagheni, Ingmar Weber, and Krishna Gummadi. 2017. Leveraging Facebook's advertising platform to monitor stocks of migrants. Population and Development Review 43, 4 (2017), 721-734.

[870] M. H. Zaki and T. Sayed. 2016. Automated cyclist data collection under high density conditions. IET Intelligent Transport Systems 10, 5 (2016), 361-369.

[871] Ahmed Zamzam and Kyri Baker. 2020. Learning optimal solutions for extremely fast AC optimal power flow. In 2020 IEEE International Conference on Communications, Control, and Computing Technologies for Smart Grids (SmartGridComm'20). IEEE, 1-6.

[872] V.Zeleňák, M. Badaničová, D. Halamova, J. Čejka, A. Zukal, N. Murafa, and G. Goerigk. 2008. Amine-modified ordered mesoporous silica: Effect of pore size on carbon dioxide capture. Chemical Engineering fournal 144, 2 (2008), 336-342.

[873] Weiliang Zeng, Tomio Miwa, and Takayuki Morikawa. 2017. Application of the support vector machine and heuristic $\mathrm{k}$-shortest path algorithm to determine the most eco-friendly path with a travel time constraint. Transportation Research Part D: Transport and Environment 57 (2017), 458-473.

[874] Haifeng Zhang and Yevgeniy Vorobeychik. 2019. Empirically grounded agent-based models of innovation diffusion: A critical review. Artificial Intelligence Review 52, 1 (2019), 707-741.

[875] Haifeng Zhang, Yevgeniy Vorobeychik, Joshua Letchford, and Kiran Lakkaraju. 2016. Data-driven agent-based modeling, with application to rooftop solar adoption. Autonomous Agents and Multi-Agent Systems 30, 6 (2016), 1023-1049.

[876] Jiansong Zhang and Nora M. El-Gohary. 2015. Automated information transformation for automated regulatory compliance checking in construction. Journal of Computing in Civil Engineering 29, 4 (2015), B4015001.

[877] Lu Zhang, Jianjun Tan, Dan Han, and Hao Zhu. 2017. From machine learning to deep learning: Progress in machine intelligence for rational drug discovery. Drug Discovery Today 22, 11 (2017), 1680-1685.

[878] Tao Zhang and William J. Nuttall. 2012. An agent-based simulation of smart metering technology adoption. International fournal of Agent Technologies and Systems 4, 1 (2012), 17-38.

[879] Wenwen Zhang, Caleb Robinson, Subhrajit Guhathakurta, Venu M. Garikapati, Bistra Dilkina, Marilyn A. Brown, and Ram M. Pendyala. 2018. Estimating residential energy consumption in metropolitan areas: A microsimulation approach. Energy 155 (2018), 162-173.

[880] Xiao Zhang, Gabriela Hug, J. Zico Kolter, and Iiro Harjunkoski. 2016. Model predictive control of industrial loads and energy storage for demand response. In 2016 IEEE Power and Energy Society General Meeting (PESGM'16). IEEE, 1-5.

[881] Zidong Zhang, Dongxia Zhang, and Robert C. Qiu. 2019. Deep reinforcement learning for power system applications: An overview. CSEE Journal of Power and Energy Systems 6, 1 (2019), 213-225.

[882] Jie Zhao, Bertrand Lasternas, Khee Poh Lam, Ray Yun, and Vivian Loftness. 2014. Occupant behavior and schedule modeling for building energy simulation through office appliance power consumption data mining. Energy and Buildings 82 (2014), 341-355.

[883] Jianing Zhao, Daniel M. Runfola, and Peter Kemper. 2017. Quantifying heterogeneous causal treatment effects in world bank development finance projects. In Machine Learning and Knowledge Discovery in Databases. Yasemin Altun, Kamalika Das, Taneli Mielikäinen, Donato Malerba, Jerzy Stefanowski, Jesse Read, Marinka Žitnik, Michelangelo Ceci, and Sašo Džeroski (Eds.). Springer International Publishing, Cham, 204-215.

[884] Xiping Zheng, Qiang Guo, Zenglu Li, and Ting Zhang. 2018. Optimal choice of enterprise's production strategy under constraints of carbon quota. International fournal of Computational Intelligence Systems 11, 1 (2018), 1268-1277.

[885] Yu Zheng. 2015. Methodologies for cross-domain data fusion: An overview. IEEE Transactions on Big Data 1, 1 (2015), $16-34$.

[886] Yu Zheng, Licia Capra, Ouri Wolfson, and Hai Yang. 2014. Urban computing: Concepts, methodologies, and applications. ACM Transaction on Intelligent Systems and Technology 5, 3 (2014), 1-55.

[887] Jianguo Zhou, Xuechao Yu, and Xiaolei Yuan. 2018. Predicting the carbon price sequence in the shenzhen emissions exchange using a multiscale ensemble forecasting model based on ensemble empirical mode decomposition. Energies 11, 7 (2018), 1907.

[888] L. Zhou and G. Wu. 2018. An overload behavior detection system for engineering transport vehicles based on deep learning. In American Institute of Physics Conference Series.

[889] Bangzhu Zhu and Julien Chevallier. 2017. Carbon price forecasting with a hybrid Arima and least squares support vector machines methodology. In Pricing and Forecasting Carbon Markets. Springer, 87-107.

[890] Bangzhu Zhu, Dong Han, Ping Wang, Zhanchi Wu, Tao Zhang, and Yi-Ming Wei. 2017. Forecasting carbon price using empirical mode decomposition and evolutionary least squares support vector regression. Applied Energy 191 (2017), 521-530. 
[891] Bangzhu Zhu, Ping Wang, Julien Chevallier, and Yiming Wei. 2015. Carbon price analysis using empirical mode decomposition. Computational Economics 45, 2 (2015), 195-206.

[892] Bangzhu Zhu, Shunxin Ye, Ping Wang, Kaijian He, Tao Zhang, and Yi-Ming Wei. 2018. A novel multiscale nonlinear ensemble leaning paradigm for carbon price forecasting. Energy Economics 70 (2018), 143-157.

[893] Xiao Xiang Zhu, Devis Tuia, Lichao Mou, Gui-Song Xia, Liangpei Zhang, Feng Xu, and Friedrich Fraundorfer. 2017. Deep learning in remote sensing: A comprehensive review and list of resources. IEEE Geoscience and Remote Sensing Magazine 5, 4 (2017), 8-36.

[894] C. Lawrence Zitnick, Lowik Chanussot, Abhishek Das, Siddharth Goyal, Javier Heras-Domingo, Caleb Ho, Weihua Hu, Thibaut Lavril, Aini Palizhati, Morgane Riviere, Muhammed Shuaibi, Anuroop Sriram, Kevin Tran, Brandon Wood, Junwoong Yoon, Devi Parikh, and Zachary Ulissi. 2020. An introduction to electrocatalyst design using machine learning for renewable energy storage. arXiv preprint arXiv:2010.09435 (2020).

[895] M. D. Zoback and S. M. Gorelick. 2012. Earthquake triggering and large-scale geologic storage of carbon dioxide. Proceedings of the National Academy of Sciences 109, 26 (2012), 10164-10168.

[896] Han Zou, Yuxun Zhou, Jianfei Yang, and Costas J. Spanos. 2018. Towards occupant activity driven smart buildings via WiFi-enabled IoT devices and deep learning. Energy and Buildings 177 (2018), 12-22.

[897] Han Zou, Yuxun Zhou, Jianfei Yang, and Costas J. Spanos. 2019. Unsupervised WiFi-enabled IoT device-user association for personalized location-based service. IEEE Internet of Things fournal 6, 1 (2019), 1238-1245.

[898] Stiffi Zukhrufany. 2018. The Utilization of Supervised Machine Learning in Predicting Corrosion to Support Preventing Pipelines Leakage in Oil and Gas Industry. Master's thesis. University of Stavanger, Norway.

Received October 2020; revised May 2021; accepted August 2021 